\title{
Influence of gene modification in biological behaviors and responses of mouse lung telocytes to inflammation
}

\author{
Dongli Song ${ }^{1 \dagger}$, Menglin $\mathrm{Xu}^{1 \dagger}$, Ruixue $\mathrm{Q}^{1^{1 \dagger}}$, Ruihua Ma ${ }^{1}$, Yile Zhou ${ }^{1}$, Duojiao Wu ${ }^{1,2^{*}}$, Hao Fang ${ }^{3,4^{*}}$ \\ and Xiangdong Wang ${ }^{1,2^{*}}$ (D)
}

\begin{abstract}
Background: Telocytes play key roles in maintenance of organ/tissue function and prevention of organ injury. However, there are great challenges to investigate telocytes functions using primary telocytes, due to the difficulties of isolation, identification, and stability. The present study aims at constructing continuous cell strain of mouse lung telocyte cell line with stable characters by gene modification and investigating biological behaviors and responses of gene-modified telocytes to inflammation.

Methods: Mouse primary lung telocytes were isolated and identified using immune-labeling markers and immunoelectron microscopy. Primary telocytes were transformed with Simian vacuolating virus 40 small and large $T$ antigen (SV40). Biological characters, behaviors morphology, and proliferation of those gene-modified telocytes were defined and monitored dynamically for 50 generations, as compared with primary lung telocytes. Cell cycle of mouse primary lung telocytes or gene-modified telocytes was detected by flow cytometry.

Results: Gene modified telocytes of generations 5, 10, 30 and 50 were observed with telopodes and also showed CD34 and ckit positive. Multiple cellular morphology were also observed on telocyte cell-line under monitor of celliq and enhanced cell proliferation were showed. SV40 transduction was also reduced apoptosis and increased the ratio of $S$ and $G 2$ phases in telocyte cell-line.

Conclusion: We successfully constructed mouse lung telocyte cell-line which maintained the biological properties and behaviors as primary telocytes and could responses to inflammation induced by LPS. Thus, gene-modified lung telocytes, Telocyte Line, would provide a cell tool for researchers exploring the roles and applications of telocytes involved in physiological and pathological states in future.
\end{abstract}

Keywords: Telocytes, Gene modification, Lung, SV40, Transformation

\section{Background}

Telocytes (TCs) have been found widely spreaded in a large number of organs and tissues of mammals,

\footnotetext{
*Correspondence: wuduojiao@126.com; drfanghao@163.com; xdwang@fuccb.com

${ }^{\dagger}$ Dongli Song, Menglin Xu and Ruixue Qi contributed equally as co-first authors.

1 Zhongshan Hospital Institute of Clinical Science, Shanghai Institute of Clinical Bioinformatics, Shanghai Engineering Research for Al Technology for Cardiopulmonary Diseases, Shanghai, China

${ }^{3}$ Department of Anesthesiology, Zhongshan Hospital, Shanghai, China Full list of author information is available at the end of the article
}

including atrial and ventricular myocardium, bladder, lung skeletal muscle, gastrointestinal tract, eyes, and others. TCs communicate with neighboring cells by homo- and heterocellular contacts and transfer genetic information and signaling molecules to influence other cells [1, 2]. Shoshkes-Carme et al. [3] recently demonstrated that TCs can be the major source of Wnt signaling, and play dependent roles in proliferation of intestinal stem cells and epithelial renewal. This particular study provides solid evidence that forkhead box L1-positive TCs contribute to the formation of the subepithelial plexus of the intestine, support the entire epithelium, and 
provide niche signals to intestinal stem cells, by producing Wnt ligands and maintaining Wnt signal pathway. Although TCs are often allocated within the stem cell niche, pulmonary TCs have different genetic profiles and biological functions from stem cells, fibroblasts, alveolar type II cells, airway basal cells, proximal airway cells, and $\mathrm{T}$ cells from bronchial lymph nodes or from lungs, respectively [4-7]. TCs functionally and constructively support and connect tissue cells, function as signal massage carriers, and benefit gene and cell therapies [8-12].

Gene modification is considered as a potential therapy to genetically prevent and treat diseases, even though a large number of factors still need to furthermore clarified, e.g. safety, efficacy, and complexity. With rapid development of gene editing technology, the precision and efficacy of gene modification are improved significantly [13, 14]. Safety profiles of gene modification as part of limitations require more preclinical and clinical evidence. The present study investigates potential effects of gene modification on morphological phenomes, biological behaviors and functions, as well as responses to inflammation in lung TCs. An anti-aging gene from Simian vacuolating virus 40 was transferred as the target gene into primary TCs isolated from mouse lungs to improve the longevity of cells, since primary TCs hardly survive in the in vitro system for a few weeks or months [5-7].

\section{Materials and methods}

\section{TCs isolation and primary cell culture}

Mice were provided by Animal Facility in Biomedical Research Center of Zhongshan Hospital, Fudan University. This study was approved by the Fudan University Ethical Committee for animal experiments.

Mouse lung TCs were isolated and prepared as escribed previously [4]. In brief, female $\mathrm{BABL} / \mathrm{c}$ mice aged 6-8 weeks were used and anaesthetized. The trachea and lung tissues were isolated and collected into sterile tubes, containing Dulbecco's Modified Eagle's Medium plus F12 (DMEM/F12) integration (Gibco, NY, USA), supplemented with $100 \mathrm{UI} / \mathrm{ml}$ penicillin, $0.1 \mathrm{mg} / \mathrm{ml}$ streptomycin (Sigma-Aldrich Shanghai Trading Co Ltd., Shanghai, China). Samples were placed on ice and transported to the cell culture laboratory within $30 \mathrm{~min}$. The tissues were cut into about $1 \times \mathrm{mm}^{3}$ in sterile DMEM/F12 and incubated in $10 \mathrm{mg} / \mathrm{ml}$ collagenase type II (Sigma-Aldrich, St. Louis, MO, USA) and $2000 \mathrm{U} / \mathrm{ml}$ deoxyribonuclease I (Sigma-Aldrich) on an orbital shaker for $4 \mathrm{~h}$, at $37^{\circ} \mathrm{C}$. Cell suspension was separated by 40 -m-diameter cell strainer (BD Falcon, NJ, USA), and dispersed cells were collected by centrifugation. Cells were resuspended in DMEM/ F12, supplemented with $10 \%$ fetal calf serum, $100 \mathrm{UI} /$ $\mathrm{ml}$ penicillin, $0.1 \mathrm{mg} / \mathrm{ml}$ streptomycin (Sigma-Aldrich), and cultured in a incubator, with $5 \% \mathrm{CO}_{2}$ in air, at $37{ }^{\circ} \mathrm{C}$, for $30 \mathrm{~min}$, to wipe off most fibroblasts. Cell suspension was transferred to other flakes and cultured for another $12 \mathrm{~h}$ before changing culture medium. Culture medium was changed every $48 \mathrm{~h}$. Cells were examined by phase contrast microscope, under an inverted Olympus phase contrast microscope $(1 \times 51$; Tokyo, Japan $)$.

\section{Lentivirus construction and infection}

Lentivirus particles containing the small and large $\mathrm{T}$ antigen of anti-aging gene from Simian vacuolating virus 40 (SV40) gene were constructed (HanyinCo., Shanghai, China) according to the former articles [15]. The oligo (listed $5^{\prime}-3^{\prime}$ ) SV40-F: CCG GAATTCATGGATAAA GTTTTAAACAGAGAGGAATC and SV40-R: GCTCTA GATTACAAGTCCTCTTCAGAAATGAG. TCs were infected on 6-well plates for $12 \mathrm{~h}$ at $37^{\circ} \mathrm{C}$, replaced fresh medium, and harvested for qPCR analysis to determine the SV40 mRNA expression level after being cultured for $96 \mathrm{~h}$ at $37^{\circ} \mathrm{C}$.

\section{RNA extraction and PCR}

Primary TCs and TCs transformed with SV40 (TCs ${ }^{\mathrm{SV} 40}$ ) were seeded in 24-well plates with a density of $10^{4}$ cells/ well and cultured for $24 \mathrm{~h}$ at $37{ }^{\circ} \mathrm{C}$. Cells were washed thrice with PBS, and total RNA was isolated and transcribed into single-stranded cDNA using the 1st Strand cDNA Synthesis Kit for RT-PCR (AMV, Roche) following the recommendations of the manufacturer.

cDNA was synthesized from $1 \mu \mathrm{g}$ of total RNA using PrimeScript $^{\circledR}$ RT reagent Kit (Takara Bio Inc., Shiga, Japan). PCR was performed with $1 \mu \mathrm{l}$ of cDNA using GoTaq polymerase (Promega) for 25 cycles with specific primers for SV40. PCR reaction products were resolved and stained with Gelred (Biotium Inc., Newark, USA).

\section{Detection of cell bio-behaviors}

The bio-behaviors of TCs and $\mathrm{TCs}{ }^{\mathrm{SV} 40}$ were recorded and analyzed using a Cell-IQ cell culturing platform (ChipMan Technologies, Tampere, Finland), equipped with a phase-contrast microscope (Nikon CFI Achromat phase contrast objective with 10 magnification) and a camera20. The bio-behaviors, including cell proliferation, division, death, cell morphology, and cell movement, can be monitored and recorded as time-lapse data by this Cell-IQ system uses machine vision technology. Images were captured at about $30 \mathrm{~min}$ intervals for $48 \mathrm{~h}$. Analysis was carried out with a freely distributed Image software (McMaster Biophotonics Facility, Hamilton, ON), using the Manual Tracking plugin created by Fabrice Cordelie'res (Institute Curie, Orsay, France). 


\section{RNA microarrays and long non-coding RNA (IncRNA) classification pipeline}

RNA microarrays and lncRNA classification pipeline were tested in primary $\mathrm{TCs}$ or $\mathrm{TCs}^{\mathrm{SV} 40}$. Briefly, total RNA was collected using NucleoSpin ${ }^{\circledR}$ RNA Plus according the manufacturer's protocol (Macherey-Nagel, Inc., Düren, Germany). Microarray and quality controls of gene expression profiling were performed after RNA and cDNA amplifications, using the GeneChip ${ }^{\circledR}$ Human Transcriptome Array 2.0 gene chip (Affymetrix, Inc., UK) with 67,528 genes. Gene expression data from each group were analyzed using Expression Console and Transcriptome Analysis Console 3.0.0.466 (Affymetrix). The differentially expressed mRNA and lncRNAs were used for a hierarchical clustering analysis (HCA) in Cluster and TreeView (https://sourceforge.net/projects/jtreeview/ files/).

\section{Immunofluorescent staining}

Double immunofluorescent staining for CD34 and vimentin was performed as previously reported [21]. In brief, primary $\mathrm{TCs}$ or $\mathrm{TCs}^{\mathrm{SV} 40}$ in $1,5,10,30$, or 50 generations were load and cultured on glass bottom cell culture dishes with $20 \mathrm{~mm}$ diameter glass (NEST, Nanjing, China) and were fixed in $4 \%$ paraformaldehyde containing $0.05 \%$ Triton-X-100 for $20 \mathrm{~min}$. The cells were washed thrice with PBS and blocked in 5\% bovine serum albumin (BSA) for $1 \mathrm{~h}$ and incubated overnight at $4{ }^{\circ} \mathrm{C}$ with mouse anti-CD34 antibody and goat anti-vimentin antibody or rabbit anti-ckit antibody (1:200 dilution; Abcam, Cambridge, UK) diluted in 1\% BSA in PBS. Cells were washed in PBS thrice and incubated with PE conjugated anti-goat secondary antibodies and FITC conjugated anti-rabbit secondary antibodies and/or FITC conjugated anti-mouse secondary antibodies (1:200 dilution; Jackson ImmunoResearch, USA). The nuclear were marked by DAPI staining, according to the manufacture's instruction (KeyGEN BioTECH, Nanjing, China).

\section{Transmission electron microscopy}

The ultrastructure of cells were observed under transmission electron microscopy (TEM) as previously reported (14). In brief, primary TCs or $\mathrm{TCs}^{\mathrm{SV} 40}$ in $1,5,10,30$, and 50 generations were cultured, collected, and fixed in $4 \%$ glutaraldehyde $\left(\mathrm{pH} 7.3,4{ }^{\circ} \mathrm{C}\right)$ for $4 \mathrm{~h}$. Cells were then washed with $0.1 \mathrm{M}$ cacodylate buffer and post-fixed with $1 \%$ osmium tetroxide in $0.1 \mathrm{M}$ cacodylate buffer $\left(\mathrm{pH} 7.3,4{ }^{\circ} \mathrm{C}\right)$. After fixing, cells were dehydrated in a graded series of ethanol, impregnated in propylene oxide (immersed overnight in a mixture of propylene oxide and Epon 812 resin), and embedded in Epon 812. Ultrathin sections at $70 \mathrm{~nm}$ were cut on a Leica LKB-II (Nußloch, Germany), collected on Formvar-coated copper grids, stained with uranyl acetate and lead citrate, and observed at an acceleration voltage of $80 \mathrm{kV}$ electron microscope (JEOL JEM-1230, Tokyo, Japan).

\section{Immunoelectron microscopy}

Ultrathin sections were prepared and collected on nickel grids. Immunolabeling staining for CD34/Vimentin and ckit/platelet-derived growth factor receptor $\alpha$ (PDGFR- $\alpha$ ) was used as previously reported [22]. In brief, sections were incubated in $50 \mathrm{mM}$ Glycine for $30 \mathrm{~min}$ and washed in Ultra-pure Water thrice for $5 \mathrm{~min}$. Sections were etched in $1 \%$ sodium periodate for 10 min following washing in Ultra-pure water. Sections were incubated in the blocking buffer for $20 \mathrm{~min}$ and labeled with rabbit anti-ckit antibody, mouse anti-CD34 antibody, goat antivimentin antibody and/or rat anti PDGFR- $\alpha$ antibody (1:200 dilution; Abcam) at $4{ }^{\circ} \mathrm{C}$ for $24 \mathrm{~h}$. The nickel grids were washed in PBS for 5 min 12 times, blocked within $1 \% \mathrm{BSA}$ for $20 \mathrm{~min}$, and incubated with $10 \mathrm{~nm}$ gold conjugated anti-goat secondary antibodies, $18 \mathrm{~nm}$ gold conjugated anti-mouse secondary antibodies, $25 \mathrm{~nm}$ gold conjugated anti-rat secondary antibodies, and/or $40 \mathrm{~nm}$ gold conjugated anti-rabbit secondary antibodies (1:200 dilution; Abcam) for $2 \mathrm{~h}$. Nickel grids were dried on filter paper and observed with transmission electronic microscopy (TEM). The staining controls included cells stained only with the second antibodies with gold labelling or the first antibodies.

\section{Cell cycle assay}

Propidium iodide (PI) staining was used for cell cycle analysis of primary $\mathrm{TCs}$ and $\mathrm{TCs}^{\mathrm{SV} 40}$ as described in manufacturer. In brief, cells were collected and fixed in $75 \%$ ethanol at $4{ }^{\circ} \mathrm{C}$ for overnight. After centrifuging and washing, staining buffer (BD Pharmingen, NJ, USA) with $0.5 \mathrm{ml} \mathrm{PI} / \mathrm{RNase}$ was added to each tube for $15 \mathrm{~min}$ at room temperature. Samples were examined with a fluorescence-activated cell sorting flow cytometer (FACS Aria II, Becton, Dickinson and Company, NJ, USA) and DNA histograms were analyzed with Flowjo 7.6.1 software. Each test was repeated thrice.

\section{Statistics}

Data were expressed as mean \pm SEM analyzed using SPSS Statistics 20 (IBM, Chicago, USA). Statistical differences between two groups were compared by t-test. Statistical differences among more than two groups were determined using ANOVA. p value less than 0.05 was considered significant. 


\section{Results}

Telopodes (Tps) as one of characteristic structures of TCs were demonstrated in Fig. 1a, b. The c-kit/CD117, CD34 and vimentin in primary lung TCs were detected and shown in Fig. 1c, d, f-h. TEM tomography also showed that TCs have narrow and flat cellular prolongations surrounding other TCs in Fig. 1i, j. Mitochondria and endoplasmic reticulums in cytoplasm and nuclear of TCs were shown (Fig. 1k). Cytomembrane were also shown clearly under TEM (Fig. 11).

The quality of SV40 gene insert in $\mathrm{TCs}^{\mathrm{SV} 40}$ were defined with SV40 mRNA expression and shown in Fig. 2a. Characteristics of telocytes in $\mathrm{TCs}^{\mathrm{SV} 40}$ were identified and telopodes of $\mathrm{TCs}^{\mathrm{SV} 40}$ were observed and recorded in Fig. $2 \mathrm{~b}$. The positive staining of vimentin and CD34 was detected in primary lung TCs and TCs ${ }^{\mathrm{SV} 40}$, as presented in Fig. 2c, d.

In order to demonstrated the immortalization and stability of $\mathrm{TCs}^{\mathrm{SV} 40}$, telopodes were observed in $\mathrm{TCs}^{\mathrm{SV} 40}$ cultured for 1, 5, 10 and 20 generations SV40 mRNA positively expressed in $\mathrm{TCs}^{\mathrm{SV} 40}$ cells through generations, as compared with primary lung TCs or SV40-negative cells (Fig. 2a). Characteristics of $\mathrm{TCs}^{\mathrm{SV} 40}$ were furthermore evaluated at $2,5,10$, or 20 generations, respectively, including telopodes (Fig. 2b1-b4), positive staining of c-kit (Fig. 2c1-c4), positive staining of CD34 (Fig. 2d1$\mathrm{d} 4)$, or positive staining of both CD34 and vimentin at 2 (e1), 5 (e2), 10 (e3), 20 (e4), 30 (e5), 40 (e6), 50 (e7), or 60 generations (e8), where cell nuclei were stained with DAPI. Dynamic alterations of TCs ${ }^{\mathrm{SV} 40}$ at 2, 5, 10, 20, 30, 50 , or 60 generations were recorded automatically each $30 \mathrm{~min}$ for $48 \mathrm{~h}$ and cell morphological phenomes were presented each $12 \mathrm{~h}$, respectively, in Additional file 1:
Figure S1. Figure 3 demonstrated that immuno-positive staining of the vimentin labeled with particle diameter at $10 \mathrm{~nm}$, CD34 at18 nm, platelet-derived growth factor receptor $\alpha$ (PDGFR $\alpha)$ at $25 \mathrm{~nm}$, or ckit at $40 \mathrm{~nm}$ in lung $\mathrm{TCs}^{\mathrm{SV} 40}$ under transmission electronic microscopy. Tomography of TCs was taken immediately after transfer with SV40 tomography, to show mitochondria-rich cytoplasm and surrounded nucleus as well as telopodes with mitochondria (Fig. 3a). PDGFR $\alpha$, ckit, or vimentin and CD34 were easily detected in TCs ${ }^{\mathrm{SV} 40}$ at 2 (b, c, d), 5 (e, f), $10(g, h), 30(i, j)$, or 50 generations $(k, l)$, respectively. Those findings of transmission electronic microscopy tomography expression demonstrated that the characteristic structures and expressions of specific markers could be stable and consistent until generation 60 .

The profiles of transcriptional factor and lncRNA genes between primary lung TCs and $\mathrm{TCs}^{\mathrm{SV} 40}$ were compared and listed in Tables 1 and 2, and the hotmap was shown in Fig. 4. As compared with purified primary lung TCs, 367 or 621 genes were up- or down-regulated in purified $\mathrm{TCs}^{\mathrm{SV} 40}, 668$ or 890 genes in non-purified lung $\mathrm{TCs}^{\mathrm{SV} 40}$, or 36 genes up-regulated in non-purified primary lung TCs. As compared with non-purified TCs ${ }^{\mathrm{SV} 40}, 71$ or 116 genes were up- or down-regulated in purified $\mathrm{TCs}^{\mathrm{SV} 40}$. Details of transcriptional factor and lncRNA gene profiles were listed in Additional file 2: Table S1. COL3A1 and SFRP2, which code alpha 1 type III collagen and secreted frizzled-related protein 2 , respectively, significantly up-regulated in purified $\mathrm{TCs}^{\mathrm{SV} 40}$, as compared with purified primary lung TCs. The patterns of transcriptional factor and lncRNA gene profiles were shown in Fig. $4 \mathrm{a}, \mathrm{b}$, respectively). The top 5 transcriptional factor genes are COL3A1, SLIT3, FST, NNAT and PCDH17,
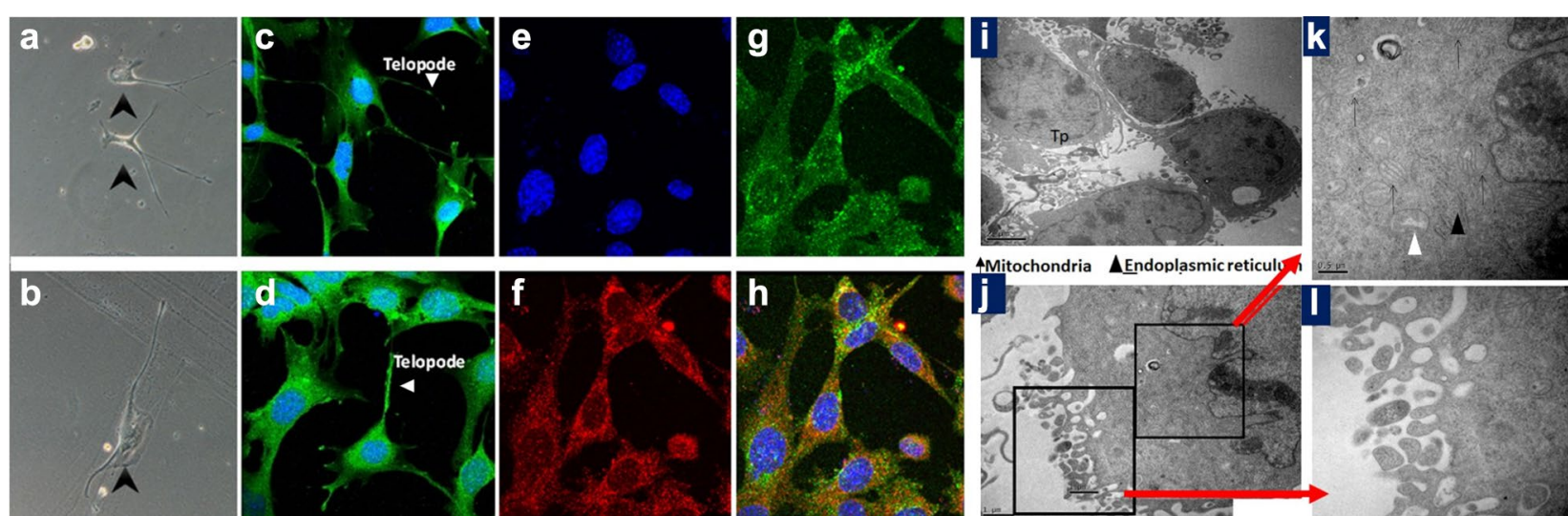

Fig. 1 Characteristics of primary lung telocytes (TCs). a, b Telocytes with telopodes (black arrow heads), c positive staining of c-kit (green), and telopodes (white arrow heads), d positive staining CD34 (green), and telopodes (white arrow heads), e nuclear staining with DAPI, f positive staining of vimentin (red), $\mathbf{g}$ positive staining of CD34 (green), $\mathbf{h}$ merge of DAPI, vimentin, and CD34, i telopode ultrastructure (Tp), $\mathbf{j}$ organelle and cell membrane, $\mathbf{k}$ mitochondrial (black arrows), mitochondrial vacuoles (white arrow head) and endoplasmic reticulum (black arrow head) in the cytoplasm, and I microvilli under TEM 


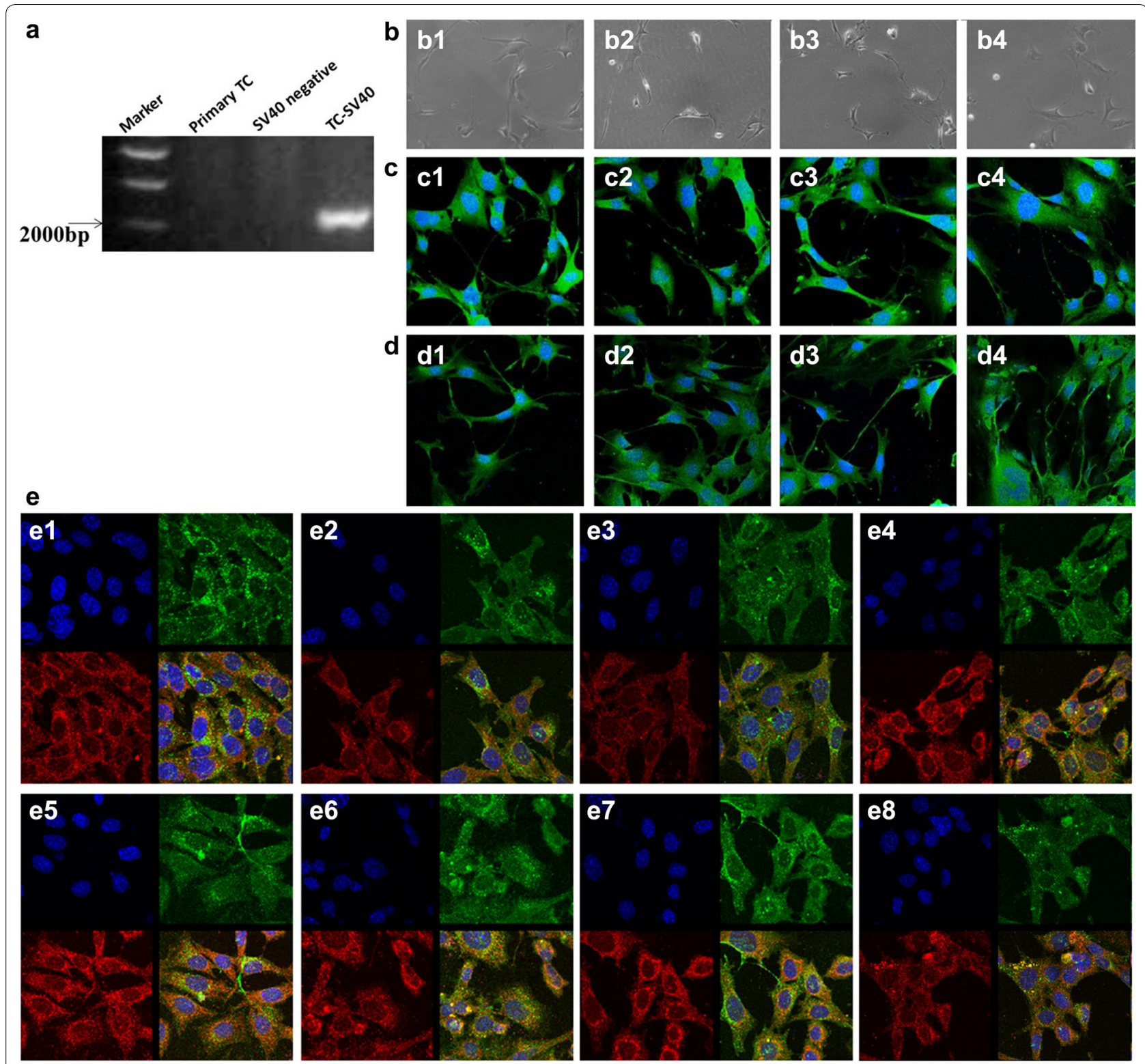

Fig. 2 TCs characteristics of lung TCs transferred with SV40 $\left(\mathrm{TCS}^{\mathrm{SV} 40}\right.$ ). a SV40 mRNA expression of $T C s^{\mathrm{SV} 40}$ cells, as compared with primary lung TCs or SV40-negative cells, $\mathbf{b}$ telopodes of TCs ${ }^{\mathrm{SV} 40}$ at 2 (b1), 5 (b2), 10 (b3), or 20 generations (b4), c positive staining of c-kit (green) in TCs $s^{\mathrm{SV} 40}$ at 2 (c1), 5 (c2), 10 (c3), or 20 generations (c4), d positive staining of CD34 (green) in TCs ${ }^{\mathrm{VV} 40}$ at 2 (d) $), 5$ (d2), 10 (d3), or 20 generations (d4), and e positive staining of CD34 (green) and vimentin (red) in TCs ${ }^{\mathrm{SV} 40}$ at 2 (e1), 5 (e2), 10 (e3), 20 (e4), 30 (e5), 40 (e6), 50 (e7), or 60 generations (e8), where cell nuclei were stained with DAPI

respectively. The top 5 lncRNA genes are TC17000728. hg.1, TC06001978.hg.1, TC08000302.hg.1, TC07001784. hg.1 and TC03003114.hg.1, respectively.

We noticed the proliferation rate of lung $\mathrm{TCs}^{\mathrm{SV} 40}$ at all generations that we detected was significantly higher than that of primary lung $\mathrm{TCs}^{\mathrm{SV} 40}$ (Fig. 5a), of which the highest proliferation rate was observed in lung $\mathrm{TCs}^{\mathrm{SV} 40}$ at generation 2, while the lowest in at generation 50. Lung $\mathrm{TCs}^{\mathrm{SV} 40}$ were mainly located in cell cycle phases of $\mathrm{S}$ and
G2, while primary lung TCs in sub-G1 phase (Fig. 5b). Furthermore, Fig. $5 \mathrm{c}$ demonstrates the alterations of cell cycle phases of Lung $\mathrm{TCs}^{\mathrm{SV} 40}$ compared with primary lung TCs. We found that the number of proliferation (Fig. 6b1-c1) or differentiated $\mathrm{TCs}^{\mathrm{SV} 40}$ (Fig. 6b3) significantly decreased in LPS administration at 1 or $0.1 \mu \mathrm{g} /$ $\mathrm{ml}$, respectively. LPS at $1 \mu \mathrm{g} / \mathrm{ml}$ caused a significant cell death (Fig. 6b2). Administration with SB216763 significantly inhibited the cell death (Fig. 6b2) or differentiated 


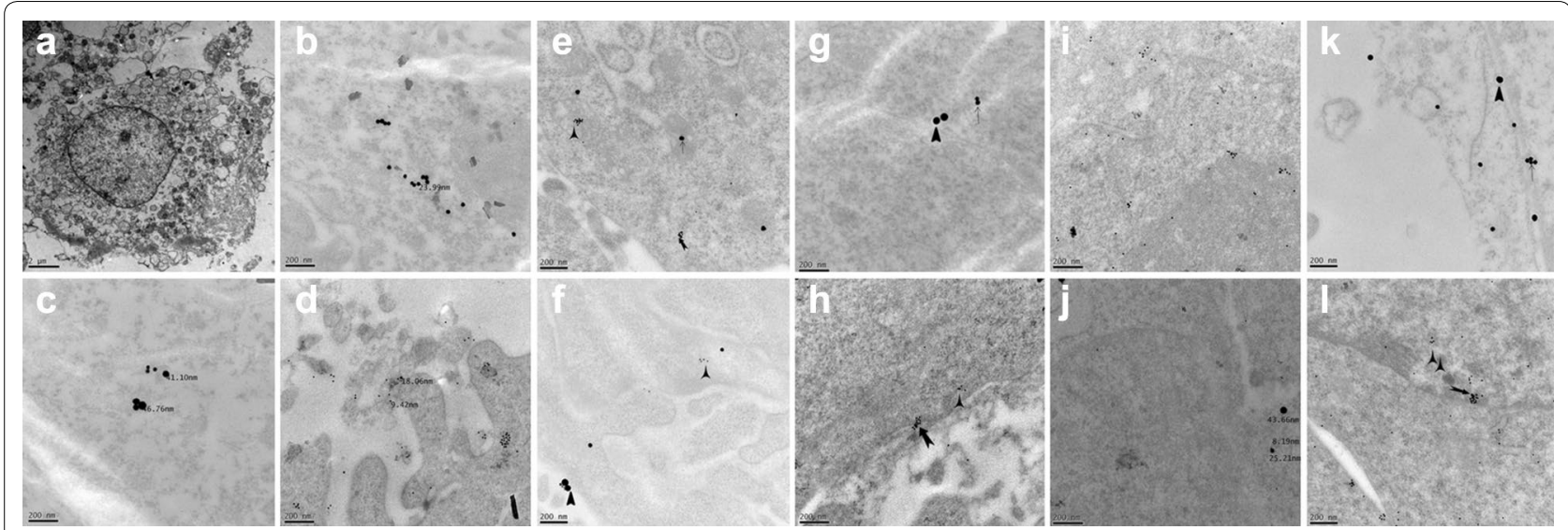

Fig. 3 Immuno-staining transmission electronic microscopy tomography of vimentin, CD34, platelet-derived growth factor receptor a (PDGF), or ckit of lung TCs transferred with SV40 (TCs ${ }^{\text {SV40 }}$ ). a TCsSV40 tomography, and positive staining of vimentin (, $\left.\left.10 \mathrm{~nm}\right), \mathrm{CD} 34(\mathbf{n}), 18 \mathrm{~nm}\right), \mathrm{PDGF}(\boldsymbol{\Lambda}$, $25 \mathrm{~nm})$, or ckit $(40 \mathrm{~nm})$ in TCs ${ }^{5 V 40}$ at $2(\mathbf{b}-\mathbf{d}), 5(\mathbf{e}, \mathbf{f}), 10(\mathbf{g}, \mathbf{h}), 30(\mathbf{i}, \mathbf{j})$, or 50 generations $(\mathbf{k}, \mathbf{l})$

number (Fig. 6b3) of TCs ${ }^{\mathrm{SV} 40}$ treated with LPS at doses of 1.0 or $0.1 \mu \mathrm{g} / \mathrm{ml}$, respectively, while could prevented LPS high dose-decreased proliferating as well as induced cell death (Fig. 6c1, c2). Administration with LY294002 significantly inhibited the cell death (Fig. 6b2) or differentiated number (Fig. 6b3) of $\mathrm{TCS}^{\mathrm{SV} 40}$ treated with LPS at doses of $0.1 \mu \mathrm{g} / \mathrm{ml}$, respectively, while could prevented LPS high dose-decreased proliferating as well as induced cell death (Fig. 6c1, c2). Figure 7 demonstrates that the ratio of $\mathrm{TCs}^{\mathrm{SV} 40}$ proliferation, death, and dividing after cells were challenged with vehicle or TNF $\alpha$ at different concentrations of $0.2,20$, or $200 \mu \mathrm{g} / \mathrm{ml}$ and treated with vehicle, SB216763 or LY294002. The number of proliferating cells significantly reduced $48 \mathrm{~h}$ after administration with TNF $\alpha$ at doses of $200 \mu \mathrm{g} / \mathrm{ml}$ (Fig. 7b1, c1). Cell proliferation, cell death and differentiated number analysis of LY294002 or SB216763 stimulated TCs ${ }^{\mathrm{SV} 40}$ by celliq were shown in Additional file 3: Figure S2. The representative photos of cell bio-behaviors of $\mathrm{TCs}^{\mathrm{SV} 40}$ stimulated by LPS, LY294003 and SB216763 recorded by celliq were shown in Additional file 4: Figure S3. Additional file 5: Figure S4 showed the representative photos of cell biobehaviors of $\mathrm{TCs}^{\mathrm{SV} 40}$ stimulated by TNF- $\alpha$, LY294003 and SB216763 recorded by celliq.

\section{Discussion}

TCs play an important role in the occurrence and progression of acute and chronic lung injury, asthma, and lung cancer $[1,10,12]$, responsible for the highest mortality and morbidity of patients. TCs can directly communicate with a large number of cells within organ and contribute to tissue repair and regeneration, as potential alternative of cell therapies [10]. We initially isolated and purified TCs from human lung and airway tissues
[16], and defined the special identity and genomic phenomes of lung TCs, different from lung stem cells, fibroblasts, alveolar type II cells, airway basal cells, proximal airway cells, $\mathrm{CD}^{8+} \mathrm{T}$ cells from bronchial lymph nodes, and $\mathrm{CD}^{8+} \mathrm{T}$ cells from lungs $[4,5,17]$. Our previous studies have proved the independence and specificity of human lung TCs at genomic levels and proposed TCs as a major source to connect cells, e.g. between TCs per se, or between TCs with other cells. The immunocytochemical markers of TCs include CD34, vimentin, c-kit, CD34/c-kit, CD34/vimentin, or PDGFR $\alpha$ [3, 10, 16, 17]. The specificity of TCs and telocytes-specific biomarkers for the identification are still to be furthermore defined, since there are a large number of telocyte heterogeneities on source, preparation, pathway, duration, and measurable variables. There are obvious differences of TCs among tissues and organs, dependent upon biological functions and characters of TC-connected tissues and organs. TCs in lung have the specificity of connection with air-liquid epithelial cells, tolerance to movement and pressure, and flexibility among barriers. In order to overcome those limits and difficulties, the present study develops a mouse lung telocyte cell-line by gene editing with lentivirus particles containing the anti-aging gene from Simian vacuolating virus 40 (SV40) gene. In the present study, we found morphology, immune biomarkers, and ultrastructure of SV40-positive TCs are similar and coincident with those of primary TCs directly isolated from mouse lungs or cultured for days. Dynamic observations of bio-behaviors demonstrated that $\mathrm{TCs}^{\mathrm{SV} 40}$ proliferation obviously increased, rather than cell movement. The capability of TCs ${ }^{\text {SV } 40}$ proliferation declined by increased consecutive passages and became more stable at the 50th passage, which was still significantly higher than the primary TCs. 
Table 1 Summary of mRNA expressed preferentially in primary TCs, SV40-transformed TCs, primary lung cells, and SV40-transformed primary lung cells

\begin{tabular}{|c|c|c|c|c|}
\hline Compared pairs & $U p>0$ & $U p>2$ & Down $>0$ & Down $>2$ \\
\hline $\begin{array}{l}\text { Purified } \mathrm{TCs}^{\mathrm{SV} 40} \text { vs. non- } \\
\text { purified primary lung } \mathrm{TCs}\end{array}$ & 158 & 0 & 124 & 0 \\
\hline $\begin{array}{l}\text { Non-purified lung } \mathrm{TCs}^{\mathrm{S} V 40} \mathrm{vs} \text {. } \\
\text { purified primary lung } \mathrm{TCS}\end{array}$ & 343 & 1 & 119 & 0 \\
\hline $\begin{array}{l}\text { Non-purified lung TCs SV40 } \\
\text { vs. non-purified primary } \\
\text { lung TCs }\end{array}$ & 274 & 1 & 151 & 0 \\
\hline $\begin{array}{l}\text { Purified TCs }{ }^{\mathrm{SV} 40} \text { vs. non- } \\
\text { purified lung TCs } s^{\mathrm{SV} 40}\end{array}$ & 64 & 0 & 75 & 0 \\
\hline $\begin{array}{l}\text { Purified } \mathrm{TCs}^{\mathrm{SV} 40} \text { vs. purified } \\
\text { primary lung TCs }\end{array}$ & 255 & 2 & 122 & 0 \\
\hline $\begin{array}{l}\text { Purified primary lung TCs } \\
\text { vs. non-purified primary } \\
\text { lung TCs }\end{array}$ & 0 & 0 & 23 & 0 \\
\hline
\end{tabular}

\begin{tabular}{llll}
\hline $\begin{array}{l}\text { Gene symbol } \\
\text { or probe set ID }\end{array}$ & Fold change & $p$-value & Gene feature \\
\hline
\end{tabular}

(A) Genes up/down-regulated in purified TCS ${ }^{S V 40}$ compared with nonpurified primary lung TCS

\begin{tabular}{lcll} 
COL3A1 & 1.736972 & $5.20 \mathrm{E}-05$ & Up \\
SLIT3 & 1.428832 & $5.70 \mathrm{E}-05$ & Up \\
FST & 1.757618 & $6.20 \mathrm{E}-05$ & Up \\
NNAT & 1.428018 & $6.60 \mathrm{E}-05$ & Up \\
PCDH17 & -1.82768 & $7.10 \mathrm{E}-05$ & Down \\
KIAA1199 & 1.450952 & $7.60 \mathrm{E}-05$ & Up \\
BHLHE22 & 1.431489 & $8.10 \mathrm{E}-05$ & Up \\
KLHDC10 & -1.342675 & $8.60 \mathrm{E}-05$ & Down \\
CAPN6 & -1.502786 & $9.10 \mathrm{E}-05$ & Down \\
DBC1 & 1.42647 & $9.60 \mathrm{E}-05$ & Up \\
ANGPTL7 & 1.490275 & 0.000101 & Up \\
FBN1 & 1.555173 & 0.000106 & Up \\
IGFBP7 & 1.491186 & 0.000111 & Up \\
COL5A2 & 1.521795 & 0.000116 & Up \\
SPRR1B & -1.653323 & 0.000121 & Down \\
PCDH7 & 1.645697 & 0.000126 & Up \\
MBD6 & -1.354523 & 0.000131 & Down \\
PPARGC1A & -1.46284 & 0.000135 & Down \\
SFRP2 & 1.953208 & 0.00014 & Up \\
UPRT & -1.391618 & 0.000145 & Down \\
BNC1 & 1.309024 & 0.00015 & Up \\
TNC & 1.396486 & 0.000155 & Up \\
EGR1 & -1.562517 & 0.00016 & Down \\
COL12A1 & 1.253525 & 0.000165 & Up \\
ZFP36L1 & 1.413153 & 0.00017 & Up \\
NFAT5 & -1.658072 & 0.000175 & Down \\
GFPT2 & 1.261057 & 0.00018 & Up \\
FOXP2 & 1.209035 & 0.000185 & Up \\
TCF20 & -1.334035 & 0.00019 & Down \\
STAT3 & 1.388462 & 0.000195 & Up \\
GATA6 & 1.445955 & 0.000199 & Up \\
CYP51A1 & 1.224699 & 0.000204 & Up \\
\hline & & &
\end{tabular}

Table 1 (continued)

\begin{tabular}{|c|c|c|c|}
\hline $\begin{array}{l}\text { Gene symbol } \\
\text { or probe set ID }\end{array}$ & Fold change & p-value & Gene feature \\
\hline IGF1 & 1.320664 & 0.000209 & Up \\
\hline COL1A1 & 1.467933 & 0.000214 & Up \\
\hline PCDH19 & -1.536647 & 0.000219 & Down \\
\hline VLDLR & -1.263978 & 0.000224 & Down \\
\hline HOXA2 & 1.693458 & 0.000229 & Up \\
\hline OGT & -1.620988 & 0.000234 & Down \\
\hline ANKH & -1.252673 & 0.000239 & Down \\
\hline ZNF503 & 1.323818 & 0.000244 & Up \\
\hline PCDH10 & 1.55352 & 0.000249 & Up \\
\hline HIF1A & 1.439987 & 0.000254 & Up \\
\hline TGFB1I1 & 1.228978 & 0.000259 & Up \\
\hline MED12 & -1.302994 & 0.000264 & Down \\
\hline FBLN2 & 1.27815 & 0.000268 & Up \\
\hline HMGCS1 & 1.267852 & 0.000273 & Up \\
\hline CPXM1 & 1.343796 & 0.000278 & Up \\
\hline ILDR2 & 1.398758 & 0.000283 & Up \\
\hline MID2 & -1.297559 & 0.000288 & Down \\
\hline SEMA3A & -1.339824 & 0.000293 & Down \\
\hline PTGS2 & -1.251508 & 0.000298 & Down \\
\hline BICC1 & 1.416447 & 0.000303 & Up \\
\hline KMT2D & -1.222378 & 0.000318 & Down \\
\hline NFIA & 1.44573 & 0.000323 & Up \\
\hline SMO & -1.262105 & 0.000328 & Down \\
\hline TMEM238 & 1.23793 & 0.000332 & Up \\
\hline DNAJC1 & 1.241613 & 0.000337 & Up \\
\hline $\mathrm{H} 2 \mathrm{AFY} 2$ & 1.200548 & 0.000342 & Up \\
\hline ARF6 & 1.267706 & 0.000347 & Up \\
\hline ACLY & 1.32049 & 0.000352 & Up \\
\hline SPRR2F & -1.383183 & 0.000357 & Down \\
\hline SCD & 1.264224 & 0.000362 & Up \\
\hline GPC4 & -1.211023 & 0.000367 & Down \\
\hline KLF9 & 1.351626 & 0.000372 & Up \\
\hline ITPR3 & -1.227564 & 0.000377 & Down \\
\hline $\mathrm{CDH} 11$ & 1.285389 & 0.000382 & Up \\
\hline NXN & 1.291856 & 0.000392 & Up \\
\hline MMP2 & 1.261064 & 0.000397 & Up \\
\hline GPM6B & 1.298864 & 0.000401 & Up \\
\hline GRIA3 & 1.443882 & 0.000406 & Up \\
\hline CCND2 & -1.243142 & 0.000411 & Down \\
\hline LOX & 1.244763 & 0.000416 & Up \\
\hline MSI2 & -1.254073 & 0.000421 & Down \\
\hline FAM126A & -1.296542 & 0.000426 & Down \\
\hline $\begin{array}{l}\text { TC1 1000974. } \\
\text { hg.1 }\end{array}$ & -1.348845 & 0.000431 & Down \\
\hline DOCK9 & -1.213445 & 0.000446 & Down \\
\hline TRBV21OR9-2 & -1.381268 & 0.000451 & Down \\
\hline THBS1 & 1.376867 & 0.000456 & Up \\
\hline PLK1 & 1.251123 & 0.000461 & Up \\
\hline DDX26B & -1.339568 & 0.000465 & Down \\
\hline
\end{tabular}


Table 1 (continued)

\begin{tabular}{|c|c|c|c|}
\hline $\begin{array}{l}\text { Gene symbol } \\
\text { or probe set ID }\end{array}$ & Fold change & $p$-value & Gene feature \\
\hline FRMD5 & 1.270501 & 0.00047 & Up \\
\hline ADAMTS15 & 1.347894 & 0.000475 & Up \\
\hline MBNL1 & -1.406503 & 0.00048 & Down \\
\hline AZIN1 & -1.31031 & 0.000485 & Down \\
\hline WT1 & 1.203106 & 0.00049 & Up \\
\hline COL1A2 & 1.333456 & $5.00 \mathrm{E}-04$ & Up \\
\hline ISM1 & 1.372788 & 0.000505 & Up \\
\hline PRRX1 & 1.396349 & 0.00051 & Up \\
\hline IGIP & -1.26922 & 0.000515 & Down \\
\hline TSHZ1 & 1.31218 & 0.00052 & Up \\
\hline TNPO3 & -1.26636 & 0.000525 & Down \\
\hline FAM98A & 1.240276 & 0.00053 & Up \\
\hline HSPA5 & 1.505916 & 0.000534 & Up \\
\hline PRG4 & 1.279794 & 0.000539 & Up \\
\hline UGCG & 1.205908 & 0.000554 & Up \\
\hline CDKL5 & -1.282448 & 0.000559 & Down \\
\hline HOXB8 & 1.214483 & 0.000564 & Up \\
\hline $\mathrm{RDH} 10$ & 1.230479 & 0.000569 & Up \\
\hline ARL6IP1 & 1.267388 & 0.000574 & Up \\
\hline RNF150 & 1.296804 & 0.000594 & Up \\
\hline TBPL1 & -1.345451 & 0.000598 & Down \\
\hline KLF7 & 1.240203 & 0.000603 & Up \\
\hline ID4 & 1.227502 & 0.000608 & Up \\
\hline MBTPS2 & -1.372487 & 0.000618 & Down \\
\hline AHCYL2 & -1.2362 & 0.000623 & Down \\
\hline C9orf172 & 1.232029 & 0.000628 & Up \\
\hline THBS2 & 1.234081 & 0.000633 & Up \\
\hline IER5L & 1.31756 & 0.000638 & Up \\
\hline FZD8 & 1.478565 & 0.000643 & Up \\
\hline REXO2 & 1.287723 & 0.000658 & Up \\
\hline MAN1A1 & 1.214421 & 0.000663 & Up \\
\hline PLXDC2 & 1.315137 & 0.000677 & Up \\
\hline DPYSL2 & 1.20111 & 0.000682 & Up \\
\hline INTS5 & 1.320636 & 0.000687 & Up \\
\hline ABCB7 & -1.288423 & 0.000692 & Down \\
\hline C3orf58 & 1.21307 & 0.000712 & Up \\
\hline FAM218A & 1.35927 & 0.000717 & Up \\
\hline FMR1 & 1.401071 & 0.000722 & Up \\
\hline PCDHGC5 & 1.328006 & 0.000731 & Up \\
\hline TRPS1 & -1.275263 & 0.000736 & Down \\
\hline GPC6 & 1.24421 & 0.000741 & Up \\
\hline $\begin{array}{l}\text { TC07000141. } \\
\text { hg.1 }\end{array}$ & -1.446989 & 0.000771 & Down \\
\hline SEC23A & 1.295262 & 0.000776 & Up \\
\hline ZDHHC15 & -1.240478 & 0.000786 & Down \\
\hline SMAD7 & 1.261408 & 0.000791 & Up \\
\hline GPC3 & 1.218435 & 0.000796 & Up \\
\hline TUBB4B & 1.245339 & 0.00081 & Up \\
\hline TXNIP & 1.202009 & 0.00082 & Up \\
\hline
\end{tabular}

Table 1 (continued)

\begin{tabular}{|c|c|c|c|}
\hline $\begin{array}{l}\text { Gene symbol } \\
\text { or probe set ID }\end{array}$ & Fold change & $p$-value & Gene feature \\
\hline FIGN & 1.385827 & 0.000825 & Up \\
\hline $\begin{array}{l}\text { TC02002617. } \\
\text { hg.1 }\end{array}$ & -1.775002 & 0.00084 & Down \\
\hline DTNA & -1.217215 & 0.00085 & Down \\
\hline MEIS1 & 1.369642 & 0.000855 & Up \\
\hline PLK2 & -1.202425 & 0.00086 & Down \\
\hline RBPJ & -1.218305 & 0.000864 & Down \\
\hline NPR2 & -1.309717 & 0.000869 & Down \\
\hline NID1 & 1.228805 & 0.000874 & Up \\
\hline AKT3 & -1.201507 & 0.000879 & Down \\
\hline SAT1 & -1.219609 & 0.000884 & Down \\
\hline SURF4 & 1.201325 & 0.000889 & Up \\
\hline CELF2 & 1.384512 & 0.000914 & Up \\
\hline ZBTB18 & -1.426169 & 0.000933 & Down \\
\hline TOX & 1.381994 & 0.000938 & Up \\
\hline DACT1 & 1.205543 & 0.000943 & Up \\
\hline CASK & -1.472338 & 0.000958 & Down \\
\hline KITLG & 1.267156 & 0.000963 & Up \\
\hline KMT2A & -1.28931 & 0.000968 & Down \\
\hline TRBV6-8 & -1.402432 & 0.000973 & Down \\
\hline PARP6 & -1.228703 & 0.000983 & Down \\
\hline CLMP & 1.252655 & 0.001007 & Up \\
\hline EGR3 & -1.348087 & 0.001017 & Down \\
\hline PCDHB1 & -1.305233 & 0.001022 & Down \\
\hline $\begin{array}{l}\text { TC10000913. } \\
\text { hg.1 }\end{array}$ & -1.441636 & 0.001037 & Down \\
\hline $\begin{array}{l}\text { TC01001567. } \\
\text { hg.1 }\end{array}$ & -1.297173 & 0.001066 & Down \\
\hline SLC16A2 & 1.240464 & 0.001081 & Up \\
\hline COLEC12 & 1.203956 & 0.001096 & Up \\
\hline PAPD5 & -1.251764 & 0.001101 & Down \\
\hline GABRA3 & 1.355514 & 0.001116 & Up \\
\hline LPCAT3 & 1.334572 & 0.001126 & Up \\
\hline FAM102B & -1.249464 & 0.00113 & Down \\
\hline CALR & 1.210971 & 0.00115 & Up \\
\hline CNTFR & 1.207485 & 0.00116 & Up \\
\hline HUWE1 & -1.286372 & 0.001165 & Down \\
\hline LOXL1 & 1.3066 & 0.00117 & Up \\
\hline JHDM1D & -1.223213 & 0.00119 & Down \\
\hline HPRT1 & -1.395397 & 0.001209 & Down \\
\hline HOXC8 & 1.24955 & 0.001214 & Up \\
\hline DOCK11 & -1.266513 & 0.001234 & Down \\
\hline R3HDM2 & -1.226605 & 0.001278 & Down \\
\hline TRAM1L1 & 1.237032 & 0.001283 & Up \\
\hline POLA1 & -1.316949 & 0.001288 & Down \\
\hline VPS35 & -1.217861 & 0.001298 & Down \\
\hline CALU & -1.289373 & 0.001323 & Down \\
\hline CD248 & 1.327827 & 0.001347 & Up \\
\hline THOC2 & -1.388434 & 0.001377 & Down \\
\hline
\end{tabular}


Table 1 (continued)

\begin{tabular}{|c|c|c|c|}
\hline $\begin{array}{l}\text { Gene symbol } \\
\text { or probe set ID }\end{array}$ & Fold change & p-value & Gene feature \\
\hline UCP2 & 1.214025 & 0.001387 & Up \\
\hline ETV6 & 1.275435 & 0.001396 & Up \\
\hline AP2S1 & 1.218648 & 0.001411 & Up \\
\hline PIAS1 & -1.266045 & 0.001475 & Down \\
\hline TFAP2A & -1.207326 & 0.00149 & Down \\
\hline IGFBP4 & 1.208644 & 0.0015 & Up \\
\hline DDIT3 & -1.300464 & 0.001515 & Down \\
\hline PRR9 & -1.306757 & 0.001529 & Down \\
\hline LRP1 & 1.262418 & 0.001539 & Up \\
\hline EPHA7 & 1.222492 & 0.001549 & Up \\
\hline GATM & 1.233004 & 0.001594 & Up \\
\hline MSN & -1.260531 & 0.001598 & Down \\
\hline P4HA3 & 1.253025 & 0.001623 & Up \\
\hline TAF10 & 1.23132 & 0.001628 & Up \\
\hline PDE7B & 1.265724 & 0.001643 & Up \\
\hline KLHL13 & 1.21332 & 0.001658 & Up \\
\hline ATP11C & -1.236782 & 0.001746 & Down \\
\hline HIST1H4L & 1.225746 & 0.001761 & Up \\
\hline JMJD1C & -1.223153 & 0.001766 & Down \\
\hline WNK1 & -1.347429 & 0.001791 & Down \\
\hline ATP6AP2 & -1.265769 & 0.001815 & Down \\
\hline TRBV10-1 & -1.203962 & 0.001855 & Down \\
\hline SRPX & 1.225977 & 0.001864 & Up \\
\hline FGF7 & 1.217304 & 0.001909 & Up \\
\hline TMTC2 & 1.228712 & 0.001914 & Up \\
\hline MYO1D & 1.22793 & 0.001948 & Up \\
\hline SARS & -1.329997 & 0.001968 & Down \\
\hline STRBP & -1.209603 & 0.002002 & Down \\
\hline SOX4 & 1.201564 & 0.002017 & Up \\
\hline EGR2 & -1.203287 & 0.002022 & Down \\
\hline LCE1D & -1.216676 & 0.002047 & Down \\
\hline MED14 & -1.219439 & 0.002076 & Down \\
\hline OR10P1 & -1.236803 & 0.002086 & Down \\
\hline EXT1 & 1.244359 & 0.002091 & Up \\
\hline SOCS3 & 1.34295 & 0.002101 & Up \\
\hline CPSF6 & -1.214996 & 0.00216 & Down \\
\hline PKM & 1.208686 & 0.00218 & Up \\
\hline FOSB & -1.203628 & 0.002293 & Down \\
\hline TRAPPC1 & 1.301019 & 0.002318 & Up \\
\hline SPRR2B & -1.274955 & 0.002323 & Down \\
\hline EMX2 & 1.278187 & 0.002396 & Up \\
\hline TOP1 & 1.225449 & 0.002401 & Up \\
\hline TBC1D19 & -1.224248 & 0.002406 & Down \\
\hline PTCHD1 & -1.206554 & 0.002431 & Down \\
\hline $\mathrm{CDH} 2$ & 1.221089 & 0.002441 & Up \\
\hline SPTLC2 & 1.254554 & 0.00252 & Up \\
\hline PCBP2 & -1.289738 & 0.002534 & Down \\
\hline IGKV1D-8 & -1.227643 & 0.002549 & Down \\
\hline
\end{tabular}

Table 1 (continued)

\begin{tabular}{|c|c|c|c|}
\hline $\begin{array}{l}\text { Gene symbol } \\
\text { or probe set ID }\end{array}$ & Fold change & $p$-value & Gene feature \\
\hline $\begin{array}{l}\text { TC03000927. } \\
\text { hg.1 }\end{array}$ & -1.274058 & 0.002569 & Down \\
\hline $\begin{array}{l}\text { TC13000014. } \\
\text { hg.1 }\end{array}$ & -1.205937 & 0.002633 & Down \\
\hline BRAF & -1.234126 & 0.002648 & Down \\
\hline CNOT6L & -1.272615 & 0.002658 & Down \\
\hline PAN3 & -1.295252 & 0.002707 & Down \\
\hline SETBP1 & 1.224547 & 0.002712 & Up \\
\hline SPRR1A & -1.236736 & 0.002717 & Down \\
\hline TXLNG & -1.205245 & 0.002726 & Down \\
\hline TSC22D2 & -1.307114 & 0.002761 & Down \\
\hline ATF3 & -1.205287 & 0.002776 & Down \\
\hline FUBP1 & -1.224805 & 0.002781 & Down \\
\hline DNAJC30 & 1.22357 & 0.002825 & Up \\
\hline RCN3 & 1.218967 & 0.002879 & Up \\
\hline AGFG1 & 1.246198 & 0.002884 & Up \\
\hline HNRNPA3 & 1.23159 & 0.002919 & Up \\
\hline SRPK2 & -1.200287 & 0.002943 & Down \\
\hline ATRX & -1.226812 & 0.002978 & Down \\
\hline ADAMTS6 & -1.248056 & 0.002983 & Down \\
\hline RAB1A & 1.34731 & 0.003022 & Up \\
\hline IGKV3- 11 & -1.238746 & 0.003121 & Down \\
\hline OR4C6 & 1.227941 & 0.003145 & Up \\
\hline XBP1 & 1.202697 & 0.00316 & Up \\
\hline MURC & -1.350099 & 0.003189 & Down \\
\hline SNX12 & -1.22367 & 0.003209 & Down \\
\hline $\begin{array}{l}\text { TC1 } 1000975 . \\
\text { hg.1 }\end{array}$ & -1.332179 & 0.003239 & Down \\
\hline HOXA5 & 1.302985 & 0.003268 & Up \\
\hline RBP1 & 1.216422 & 0.003396 & Up \\
\hline $\begin{array}{l}\text { TC19000375. } \\
\text { hg.1 }\end{array}$ & -1.216419 & 0.003406 & Down \\
\hline KDM6A & -1.327135 & 0.003505 & Down \\
\hline $\begin{array}{l}\text { TC06001136. } \\
\text { hg.1 }\end{array}$ & -1.438272 & 0.003628 & Down \\
\hline CAV1 & -1.206156 & 0.003643 & Down \\
\hline ACSL4 & 1.257627 & 0.003682 & Up \\
\hline LRP6 & 1.211757 & 0.00379 & Up \\
\hline HIST1H1B & 1.245633 & 0.004096 & Up \\
\hline DEFB136 & -1.244558 & 0.00415 & Down \\
\hline FBXO33 & 1.239768 & 0.004229 & Up \\
\hline TANC2 & -1.233366 & 0.004303 & Down \\
\hline USP12 & 1.334372 & 0.004475 & Up \\
\hline OR5A2 & -1.287342 & 0.004539 & Down \\
\hline $\begin{array}{l}\text { TC18000270. } \\
\text { hg.1 }\end{array}$ & 1.23255 & 0.004657 & Up \\
\hline OR51L1 & -1.224446 & 0.004948 & Down \\
\hline OR51B6 & -1.256007 & 0.004992 & Down \\
\hline PTEN & 1.249442 & 0.005116 & Up \\
\hline TMSB10 & 1.251421 & 0.005327 & Up \\
\hline
\end{tabular}


Table 1 (continued)

\begin{tabular}{lrll}
\hline $\begin{array}{l}\text { Gene symbol } \\
\text { or probe set ID }\end{array}$ & Fold change & p-value & Gene feature \\
\hline VEZF1 & -1.29591 & 0.005436 & Down \\
NFIB & 1.220599 & 0.0055 & Up \\
CCDC166 & 1.217336 & 0.005529 & Up \\
STAG2 & -1.224684 & 0.005933 & Down \\
PIAS2 & -1.200256 & 0.00648 & Down \\
ZSWIM6 & 1.222542 & 0.00651 & Up \\
ZNF281 & 1.209366 & 0.006697 & Up \\
SYT11 & 1.201281 & 0.00682 & Up \\
SCXA & -1.222753 & 0.007815 & Down \\
ALX1 & -1.230342 & 0.007992 & Down \\
EFNA5 & 1.202225 & 0.008702 & Up \\
MGC15705 & 1.234392 & 0.009229 & Up \\
GPR174 & 1.246926 & 0.009317 & Up \\
TC09000877. & -1.325127 & 0.010825 & Down \\
hg.1 & & & \\
KRTAP19-4 & 1.262139 & 0.010972 & Up \\
OR52N2 & 1.201364 & 0.011977 & Up \\
\hline
\end{tabular}

Gene symbol Fold change $\quad \mathrm{p}$-value Gene feature

(B) Genes up/down-regulated in non-purified lung $T C s^{S V 40}$ compared with purified primary lung TCS

\begin{tabular}{lcll} 
COL1A1 & 2.030789 & $5.20 \mathrm{E}-05$ & Up \\
COL1A2 & 1.647326 & $5.70 \mathrm{E}-05$ & Up \\
SYVN1 & 1.520112 & $6.20 \mathrm{E}-05$ & Up \\
COL12A1 & 1.390303 & $6.60 \mathrm{E}-05$ & Up \\
MYH10 & 1.517922 & $7.10 \mathrm{E}-05$ & Up \\
ACTA1 & 1.453697 & $7.60 \mathrm{E}-05$ & Up \\
SLIT3 & 1.501818 & $8.10 \mathrm{E}-05$ & Up \\
COL3A1 & 1.756169 & $8.60 \mathrm{E}-05$ & Up \\
FBN1 & 1.641521 & $9.10 \mathrm{E}-05$ & Up \\
COL5A2 & 1.737694 & $9.60 \mathrm{E}-05$ & Up \\
GATA6 & 1.796826 & 0.000101 & Up \\
IGFBP7 & 1.641817 & 0.000106 & Up \\
SEMA3D & 1.437537 & 0.000111 & Up \\
ANGPTL7 & 1.811625 & 0.000116 & Up \\
MEIS2 & 1.53255 & 0.000121 & Up \\
THBS1 & 1.678925 & 0.000125 & Up \\
HS6ST2 & 1.220779 & 0.00013 & Up \\
BICC1 & 1.50068 & 0.000135 & Up \\
MED12 & -1.428542 & 0.00014 & Down \\
HSPA5 & 1.887927 & 0.000145 & Up \\
PFN1 & 1.558653 & 0.00015 & Up \\
PPARGC1A & -1.432059 & 0.000155 & Down \\
NFIB & 1.535713 & 0.00016 & Up \\
RBPJ & -1.376913 & 0.000165 & Down \\
ACTN1 & 1.37672 & 0.00017 & Up \\
EXT1 & 1.57285 & 0.000175 & Up \\
TNC & 1.293769 & 0.00018 & Up \\
PCDH7 & 1.762644 & 0.000185 & Up \\
TXNIP & 1.299338 & 0.000189 & Up \\
\hline & & &
\end{tabular}

Table 1 (continued)

\begin{tabular}{|c|c|c|c|}
\hline Gene symbol & Fold change & p-value & Gene feature \\
\hline LPCAT3 & 1.43629 & 0.000194 & Up \\
\hline COL4A5 & 1.312681 & 0.000199 & Up \\
\hline NUAK1 & 1.412387 & 0.000204 & Up \\
\hline ATP1A1 & 1.303558 & 0.000209 & Up \\
\hline WT1 & 1.363849 & 0.000214 & Up \\
\hline MASP1 & -1.206501 & 0.000219 & Down \\
\hline $\mathrm{VCL}$ & 1.617321 & 0.000224 & Up \\
\hline IER5L & 1.262549 & 0.000229 & Up \\
\hline MYO1D & 1.463161 & 0.000234 & Up \\
\hline PLOD2 & 1.269697 & 0.000239 & Up \\
\hline FOXP2 & 1.290662 & 0.000244 & Up \\
\hline ATP1B1 & 1.344335 & 0.000248 & Up \\
\hline KRTAP29-1 & -1.332419 & 0.000253 & Down \\
\hline ITGAV & 1.294132 & 0.000258 & Up \\
\hline CPXM1 & 1.343008 & 0.000263 & Up \\
\hline FST & 1.55845 & 0.000268 & Up \\
\hline CTGF & 1.578183 & 0.000273 & Up \\
\hline HMGCS1 & 1.228293 & 0.000278 & Up \\
\hline PLXDC2 & 1.295572 & 0.000288 & Up \\
\hline LOXL1 & 1.332093 & 0.000293 & Up \\
\hline TM9SF4 & 1.351928 & 0.000298 & Up \\
\hline GGCX & 1.265383 & 0.000303 & Up \\
\hline INPPL1 & 1.32131 & 0.000308 & Up \\
\hline DUSP6 & 1.275474 & 0.000312 & Up \\
\hline NFIA & 1.675759 & 0.000317 & Up \\
\hline GRIA3 & 1.592221 & 0.000322 & Up \\
\hline LTBP1 & -1.275386 & 0.000332 & Down \\
\hline $\mathrm{BNC1}$ & 1.391268 & 0.000337 & Up \\
\hline FEZ1 & -1.275203 & 0.000342 & Down \\
\hline OGT & -1.599369 & 0.000347 & Down \\
\hline NXN & 1.203178 & 0.000352 & Up \\
\hline HIST1H2BM & 1.309386 & 0.000357 & Up \\
\hline $\mathrm{APH} 1 \mathrm{~A}$ & 1.273537 & 0.000362 & Up \\
\hline TMEM259 & 1.230715 & 0.000371 & Up \\
\hline THBS2 & 1.232906 & 0.000376 & Up \\
\hline ANXA6 & 1.256028 & 0.000381 & Up \\
\hline $\mathrm{CDH} 2$ & 1.2585 & 0.000391 & Up \\
\hline SEC23A & 1.343492 & 0.000401 & Up \\
\hline NNAT & 1.433848 & 0.000406 & Up \\
\hline EMX2 & 1.295739 & 0.000411 & Up \\
\hline VCAM1 & 1.215258 & 0.000416 & Up \\
\hline DNAJC1 & 1.413451 & 0.000421 & Up \\
\hline PCDH19 & -1.490493 & 0.000426 & Down \\
\hline ILDR2 & 1.412592 & 0.000431 & Up \\
\hline DOCK3 & -1.218811 & 0.000445 & Down \\
\hline DEFB128 & -1.253647 & 0.00045 & Down \\
\hline ERO1L & 1.201323 & 0.00046 & Up \\
\hline CRYBA1 & -1.209259 & 0.000465 & Down \\
\hline CELF2 & 1.399193 & 0.000475 & Up \\
\hline
\end{tabular}


Table 1 (continued)

\begin{tabular}{|c|c|c|c|}
\hline Gene symbol & Fold change & p-value & Gene feature \\
\hline ZNF503 & 1.316826 & 0.00048 & Up \\
\hline TM9SF3 & 1.337447 & 0.000485 & Up \\
\hline PTPRD & 1.337777 & 0.000494 & Up \\
\hline IGHV3-43 & -1.352419 & 0.000499 & Down \\
\hline KIAA1199 & 1.358808 & 0.000509 & Up \\
\hline NPR2 & -1.233749 & 0.000514 & Down \\
\hline SLC7A2 & 1.219152 & 0.000519 & Up \\
\hline TRBV21OR9-2 & -1.26885 & 0.000524 & Down \\
\hline TCF7L2 & 1.344321 & 0.000534 & Up \\
\hline SRPX & 1.275048 & 0.000549 & Up \\
\hline TUBG1 & 1.240509 & 0.000554 & Up \\
\hline TNFAIP1 & 1.207721 & 0.000558 & Up \\
\hline KCNAB1 & 1.21542 & 0.000563 & Up \\
\hline ARF6 & 1.224098 & 0.000568 & Up \\
\hline FBXO33 & 1.218704 & 0.000573 & Up \\
\hline PRPF19 & 1.25947 & 0.000578 & Up \\
\hline RAPH1 & 1.350071 & 0.000583 & Up \\
\hline PDIA3 & 1.527168 & 0.000593 & Up \\
\hline PBX3 & 1.328363 & 0.000598 & Up \\
\hline SEC23B & 1.264846 & 0.000603 & Up \\
\hline GABRA3 & 1.405001 & 0.000608 & Up \\
\hline ARHGAP1 & 1.548495 & 0.000617 & Up \\
\hline AP3S1 & 1.293716 & 0.000622 & Up \\
\hline NFIX & 1.300317 & 0.000627 & Up \\
\hline SPTLC2 & 1.34066 & 0.000632 & Up \\
\hline HOXC6 & 1.280515 & 0.000637 & Up \\
\hline RAB11B & 1.318414 & 0.000642 & Up \\
\hline FHL1 & 1.206581 & 0.000652 & Up \\
\hline LMO4 & 1.222458 & 0.000672 & Up \\
\hline GBF1 & 1.291975 & 0.000677 & Up \\
\hline MBD6 & -1.274947 & 0.000681 & Down \\
\hline STAT3 & 1.346345 & 0.000686 & Up \\
\hline TLN1 & 1.318005 & 0.000691 & Up \\
\hline KIDINS220 & 1.20164 & 0.000696 & Up \\
\hline ETV6 & 1.300092 & 0.000701 & Up \\
\hline ATP2A2 & 1.245839 & 0.000706 & Up \\
\hline PARVA & 1.340702 & 0.000711 & Up \\
\hline OR10D3 & -1.379974 & 0.000716 & Down \\
\hline ILK & 1.411794 & 0.000721 & Up \\
\hline PLCE1 & 1.207815 & 0.000731 & Up \\
\hline SERPINH1 & 1.307929 & 0.000741 & Up \\
\hline HIF1A & 1.377218 & 0.000745 & Up \\
\hline PDIA4 & 1.235338 & 0.000755 & Up \\
\hline MAP4K4 & 1.281043 & 0.00076 & Up \\
\hline REXO2 & 1.383088 & 0.000765 & Up \\
\hline SLC6A17 & -1.217838 & 0.00077 & Down \\
\hline PCDH17 & -1.632353 & 0.000775 & Down \\
\hline EFNA5 & 1.543491 & 0.00078 & Up \\
\hline LCE3E & -1.318245 & 0.000785 & Down \\
\hline
\end{tabular}

Table 1 (continued)

\begin{tabular}{|c|c|c|c|}
\hline Gene symbol & Fold change & $p$-value & Gene feature \\
\hline LOX & 1.263793 & 0.00079 & Up \\
\hline FERMT2 & 1.235564 & 0.000795 & Up \\
\hline NEO1 & 1.379359 & $8.00 \mathrm{E}-04$ & Up \\
\hline TENM3 & 1.236228 & 0.000819 & Up \\
\hline $\mathrm{CDH} 11$ & 1.294755 & 0.000824 & Up \\
\hline KLHDC3 & 1.275726 & 0.000829 & Up \\
\hline HMGA1 & -1.21677 & 0.000834 & Down \\
\hline SCD & 1.277895 & 0.000839 & Up \\
\hline CCND2 & -1.222991 & 0.000844 & Down \\
\hline HES1 & 1.308538 & 0.000849 & Up \\
\hline PDE7B & 1.38073 & 0.000854 & Up \\
\hline SPARC & 1.349498 & 0.000864 & Up \\
\hline SMAD6 & 1.280817 & 0.000878 & Up \\
\hline SARS & -1.260078 & 0.000883 & Down \\
\hline OSBP & 1.345413 & 0.000903 & Up \\
\hline ACTR1A & 1.256685 & 0.000913 & Up \\
\hline RUNX1T1 & -1.252339 & 0.000918 & Down \\
\hline TFAP2A & -1.255715 & 0.000927 & Down \\
\hline NRG1 & 1.200557 & 0.000937 & Up \\
\hline HOXB5 & -1.295109 & 0.000942 & Down \\
\hline HECTD1 & 1.351927 & 0.000947 & Up \\
\hline LCE1C & -1.270503 & 0.000952 & Down \\
\hline SPRED1 & 1.241191 & 0.000957 & Up \\
\hline PLAG1 & -1.268008 & 0.000967 & Down \\
\hline EFNB2 & 1.525576 & 0.000972 & Up \\
\hline HIPK3 & 1.37177 & 0.000977 & Up \\
\hline ID1 & 1.349752 & 0.000982 & Up \\
\hline GRIA4 & 1.321472 & 0.000991 & Up \\
\hline FAM168A & 1.339626 & 0.001001 & Up \\
\hline FAM98A & 1.331744 & 0.001006 & Up \\
\hline DERL2 & 1.375325 & 0.001026 & Up \\
\hline HSPA4 & 1.279904 & 0.001031 & Up \\
\hline $\mathrm{ABCA} 1$ & 1.213015 & 0.001036 & Up \\
\hline GATM & 1.418597 & 0.001041 & Up \\
\hline SLC7A11 & 1.218849 & 0.001046 & Up \\
\hline RAB6A & 1.254262 & 0.00105 & Up \\
\hline IGF1 & 1.369249 & 0.00107 & Up \\
\hline CCDC80 & 1.219878 & 0.00108 & Up \\
\hline KMT2D & -1.206701 & 0.001085 & Down \\
\hline $\mathrm{HOXC4}$ & 1.445692 & 0.00109 & Up \\
\hline PPP3CA & 1.37054 & 0.001095 & Up \\
\hline HSPA1A & 1.212978 & 0.0011 & Up \\
\hline BMPR2 & 1.217688 & 0.001105 & Up \\
\hline ACTG1 & 1.229217 & 0.00111 & Up \\
\hline ZDHHC5 & 1.288505 & 0.001114 & Up \\
\hline $\mathrm{CNIH}$ & 1.285039 & 0.001124 & Up \\
\hline UNC5B & 1.204984 & 0.001139 & Up \\
\hline CAPN6 & -1.418505 & 0.001149 & Down \\
\hline ZFP36L1 & 1.284405 & 0.001154 & Up \\
\hline
\end{tabular}


Table 1 (continued)

\begin{tabular}{|c|c|c|c|}
\hline Gene symbol & Fold change & p-value & Gene feature \\
\hline FGF18 & 1.238166 & 0.001159 & Up \\
\hline RAB1A & 1.541258 & 0.001164 & Up \\
\hline FMR1 & 1.333774 & 0.001208 & Up \\
\hline FAM188A & 1.405263 & 0.001213 & Up \\
\hline FAM168B & 1.275522 & 0.001228 & Up \\
\hline ITGB5 & 1.204294 & 0.001242 & Up \\
\hline KRTAP5-2 & -1.219252 & 0.001252 & Down \\
\hline FBLN5 & 1.287407 & 0.001262 & Up \\
\hline ACVR2A & 1.403689 & 0.001277 & Up \\
\hline ADAMTS9 & 1.226553 & 0.001292 & Up \\
\hline NETO1 & 1.219365 & 0.001301 & Up \\
\hline $\mathrm{ABI} 1$ & 1.232061 & 0.001306 & Up \\
\hline INSL6 & 1.241028 & 0.001321 & Up \\
\hline TJP1 & 1.229587 & 0.001326 & Up \\
\hline HNRNPUL2 & 1.282031 & 0.001331 & Up \\
\hline COPG1 & 1.228987 & 0.001346 & Up \\
\hline COPB1 & 1.259033 & 0.001351 & Up \\
\hline $\mathrm{DIO} 3$ & 1.28299 & 0.001356 & Up \\
\hline DYNLL2 & 1.311492 & 0.00136 & Up \\
\hline DDX26B & -1.257624 & 0.001365 & Down \\
\hline SLIT2 & 1.236976 & 0.00138 & Up \\
\hline GPC3 & 1.242766 & 0.001385 & Up \\
\hline PYGB & 1.210405 & 0.001395 & Up \\
\hline MSANTD2 & -1.247787 & 0.001405 & Down \\
\hline CYGB & 1.321169 & 0.00141 & Up \\
\hline GPC6 & 1.254278 & 0.001415 & Up \\
\hline $\begin{array}{l}\text { TC08000204. } \\
\text { hg.1 }\end{array}$ & -1.443858 & 0.001424 & Down \\
\hline STK39 & 1.37139 & 0.001429 & Up \\
\hline RAB1B & 1.242841 & 0.001439 & Up \\
\hline ELAVL2 & -1.223928 & 0.001449 & Down \\
\hline HSP90B1 & 1.375708 & 0.001474 & Up \\
\hline ACTN4 & 1.314084 & 0.001483 & Up \\
\hline HSPA1A & 1.215746 & 0.001503 & Up \\
\hline AMMECR1L & 1.245785 & 0.001508 & Up \\
\hline RND3 & 1.239337 & 0.001518 & Up \\
\hline SPRY2 & 1.233483 & 0.001523 & Up \\
\hline DPYSL2 & 1.236003 & 0.001528 & Up \\
\hline DDB1 & 1.27575 & 0.001552 & Up \\
\hline SMARCA2 & 1.262741 & 0.001567 & Up \\
\hline HDGFRP3 & 1.330639 & 0.001572 & Up \\
\hline SULF1 & 1.221198 & 0.001577 & Up \\
\hline STK19 & -1.210825 & 0.001594 & Down \\
\hline STK19 & -1.210825 & 0.001594 & Down \\
\hline UPF2 & 1.267351 & 0.001606 & Up \\
\hline TRBV4-1 & -1.237852 & 0.001621 & Down \\
\hline APP & 1.206738 & 0.001636 & Up \\
\hline AFF4 & 1.209778 & 0.001641 & Up \\
\hline MEF2A & 1.213845 & 0.001651 & Up \\
\hline EDEM3 & 1.261632 & 0.001661 & Up \\
\hline
\end{tabular}

Table 1 (continued)

\begin{tabular}{|c|c|c|c|}
\hline Gene symbol & Fold change & $p$-value & Gene feature \\
\hline HSPA1A & 1.214617 & 0.001666 & Up \\
\hline GABRA4 & 1.24602 & 0.001675 & Up \\
\hline SPTAN1 & 1.260395 & 0.001685 & Up \\
\hline TEAD1 & 1.355621 & 0.001695 & Up \\
\hline WNK1 & -1.539904 & 0.001705 & Down \\
\hline LRP6 & 1.290518 & 0.00171 & Up \\
\hline PRRX1 & 1.233827 & 0.001749 & Up \\
\hline HSPA1A & 1.213076 & 0.001759 & Up \\
\hline RAB8A & 1.200712 & 0.001769 & Up \\
\hline STIM1 & 1.32461 & 0.001779 & Up \\
\hline ERLEC1 & 1.216094 & 0.001793 & Up \\
\hline ADRB1 & 1.24843 & 0.001803 & Up \\
\hline TMSB10 & 1.475064 & 0.001808 & Up \\
\hline ALCAM & 1.207847 & 0.001857 & Up \\
\hline CYP51A1 & 1.220803 & 0.001862 & Up \\
\hline EGR3 & -1.357705 & 0.001877 & Down \\
\hline CDC27 & 1.318143 & 0.001887 & Up \\
\hline PANK3 & 1.23265 & 0.001921 & Up \\
\hline HOXA2 & 1.488536 & 0.001951 & Up \\
\hline TRBV6-8 & -1.340511 & 0.001971 & Down \\
\hline CRIM1 & 1.207809 & 0.001975 & Up \\
\hline LMAN1 & 1.205915 & 0.00198 & Up \\
\hline DEFB113 & -1.249904 & 0.001985 & Down \\
\hline FN1 & 1.284284 & 0.00199 & Up \\
\hline PRKG1 & 1.239048 & 0.00202 & Up \\
\hline $\begin{array}{l}\text { TC15001075. } \\
\text { hg.1 }\end{array}$ & -1.244805 & 0.002025 & Down \\
\hline HIST1H2BJ & 1.336659 & 0.002074 & Up \\
\hline TMEM57 & 1.269164 & 0.002094 & Up \\
\hline USP12 & 1.314548 & 0.002118 & Up \\
\hline CAMK2N2 & 1.20406 & 0.002143 & Up \\
\hline TMTC2 & 1.200876 & 0.002162 & Up \\
\hline ANAPC1 & 1.257636 & 0.002167 & Up \\
\hline C1QL3 & 1.224547 & 0.002236 & Up \\
\hline KPRP & -1.284699 & 0.002246 & Down \\
\hline PAPOLA & 1.313258 & 0.002295 & Up \\
\hline ACTC1 & 1.2396 & 0.002305 & Up \\
\hline LCE2A & -1.308229 & 0.00232 & Down \\
\hline CHD4 & 1.255055 & 0.002349 & Up \\
\hline EIF5 & 1.234533 & 0.002354 & Up \\
\hline PCDH10 & 1.328826 & 0.002379 & Up \\
\hline MYL9 & 1.277676 & 0.002404 & Up \\
\hline MUT & -1.247925 & 0.002413 & Down \\
\hline MEIS1 & 1.223628 & 0.002423 & Up \\
\hline P2RX3 & -1.225147 & 0.002438 & Down \\
\hline UBE2M & 1.345125 & 0.002458 & Up \\
\hline BMPR1A & 1.384032 & 0.002482 & Up \\
\hline RAB39B & -1.28009 & 0.002507 & Down \\
\hline MBNL2 & 1.267238 & 0.002556 & Up \\
\hline PLK1 & 1.222337 & 0.002561 & Up \\
\hline
\end{tabular}


Table 1 (continued)

\begin{tabular}{|c|c|c|c|}
\hline Gene symbol & Fold change & p-value & Gene feature \\
\hline TRGV8 & -1.279773 & 0.002586 & Down \\
\hline CNN1 & 1.208197 & 0.00261 & Up \\
\hline ZSWIM6 & 1.291355 & 0.00265 & Up \\
\hline EFNB1 & 1.235229 & 0.002679 & Up \\
\hline MAN1A1 & 1.233248 & 0.002694 & Up \\
\hline HOXC8 & 1.264716 & 0.002714 & Up \\
\hline SMAD7 & 1.275196 & 0.002763 & Up \\
\hline TRIP12 & 1.322101 & 0.002778 & Up \\
\hline ATP2B1 & 1.212402 & 0.002812 & Up \\
\hline MEX3C & 1.246507 & 0.002822 & Up \\
\hline SEC24A & 1.225516 & 0.002827 & Up \\
\hline PSMC4 & 1.238673 & 0.002837 & Up \\
\hline KRTAP22-2 & -1.253194 & 0.002846 & Down \\
\hline LOC100509638 & -1.331709 & 0.002861 & Down \\
\hline $\mathrm{KDM} 2 \mathrm{~A}$ & 1.21736 & 0.00292 & Up \\
\hline EIF4G2 & 1.346528 & 0.002945 & Up \\
\hline NOXRED1 & -1.247281 & 0.002964 & Down \\
\hline $\begin{array}{l}\text { TC02002617. } \\
\text { hg.1 }\end{array}$ & -1.384833 & 0.002989 & Down \\
\hline STT3A & 1.269875 & 0.003009 & Up \\
\hline $\begin{array}{l}\text { TC11000975. } \\
\text { hg.1 }\end{array}$ & -1.273558 & 0.003058 & Down \\
\hline APOBEC1 & -1.247386 & 0.003063 & Down \\
\hline PPP2R5E & 1.279695 & 0.003078 & Up \\
\hline FZD8 & 1.347838 & 0.003147 & Up \\
\hline HIST1H2BH & 1.399643 & 0.003176 & Up \\
\hline AP1G1 & 1.251832 & 0.003191 & Up \\
\hline C11orf73 & 1.210719 & 0.003215 & Up \\
\hline RTN3 & 1.21898 & 0.00323 & Up \\
\hline LCE1E & -1.222095 & 0.003255 & Down \\
\hline ADAM9 & 1.210498 & 0.00326 & Up \\
\hline CLINT1 & 1.294737 & 0.003294 & Up \\
\hline TRGV3 & -1.208645 & 0.003329 & Down \\
\hline P4HA3 & 1.227186 & 0.003388 & Up \\
\hline CELF1 & 1.215676 & 0.003417 & Up \\
\hline MAP4K5 & 1.258241 & 0.003422 & Up \\
\hline TARDBP & -1.229146 & 0.003486 & Down \\
\hline BMP4 & 1.239271 & 0.003511 & Up \\
\hline ACTA2 & 1.255606 & 0.003525 & Up \\
\hline SF3B3 & 1.20972 & 0.003614 & Up \\
\hline DCUN1D5 & 1.248267 & 0.003634 & Up \\
\hline OLFML2B & 1.220926 & 0.003643 & Up \\
\hline TOP1 & 1.254134 & 0.003663 & Up \\
\hline BTG1 & -1.348061 & 0.003673 & Down \\
\hline CALR & 1.300821 & 0.003703 & Up \\
\hline H3F3A & 1.228003 & 0.003742 & Up \\
\hline PUM1 & 1.232872 & 0.00383 & Up \\
\hline PSMA3 & 1.227334 & 0.003845 & Up \\
\hline $\mathrm{OR} 2 \mathrm{H} 2$ & -1.261057 & 0.003865 & Down \\
\hline FAR1 & 1.284889 & 0.003875 & Up \\
\hline
\end{tabular}

Table 1 (continued)

\begin{tabular}{|c|c|c|c|}
\hline Gene symbol & Fold change & $p$-value & Gene feature \\
\hline $\mathrm{RC} 3 \mathrm{H}_{2}$ & 1.209665 & 0.003894 & Up \\
\hline IGKV1D-37 & 1.290533 & 0.003899 & Up \\
\hline EIF4B & -1.253518 & 0.003949 & Down \\
\hline ROMO1 & 1.212986 & 0.004017 & Up \\
\hline NCKAP1 & 1.279797 & 0.004067 & Up \\
\hline HSPA2 & 1.241027 & 0.004086 & Up \\
\hline CPD & 1.254726 & 0.004091 & Up \\
\hline NPR3 & 1.205107 & 0.004131 & Up \\
\hline RAP1B & 1.300105 & 0.004165 & Up \\
\hline SEC62 & 1.322724 & 0.00419 & Up \\
\hline IGLJ4 & 1.46599 & 0.004263 & Up \\
\hline OR5A2 & -1.612969 & 0.004332 & Down \\
\hline IGLV6-57 & 1.202727 & 0.004342 & Up \\
\hline RAB21 & 1.224355 & 0.004367 & Up \\
\hline MMP14 & 1.259391 & 0.004465 & Up \\
\hline LELP1 & -1.280949 & 0.004475 & Down \\
\hline ZNF281 & 1.341512 & 0.004495 & Up \\
\hline KRTAP12-3 & -1.222558 & 0.004657 & Down \\
\hline MMD & 1.36424 & 0.004691 & Up \\
\hline TIMP3 & 1.219996 & 0.004711 & Up \\
\hline ACTL8 & -1.248052 & 0.004746 & Down \\
\hline HIST1H2BE & 1.383938 & 0.004829 & Up \\
\hline POLA1 & -1.226576 & 0.004839 & Down \\
\hline AIDA & 1.221832 & 0.004854 & Up \\
\hline $\mathrm{OR} 2 \mathrm{H} 2$ & -1.251288 & 0.004859 & Down \\
\hline PMP22 & 1.201201 & 0.004923 & Up \\
\hline KRTAP12-3 & -1.215537 & 0.004938 & Down \\
\hline SLC36A4 & 1.206965 & 0.004962 & Up \\
\hline SHOC2 & 1.224107 & 0.004972 & Up \\
\hline sox4 & 1.200056 & 0.004977 & Up \\
\hline ISM1 & 1.253651 & 0.005031 & Up \\
\hline CSRP2 & 1.210735 & 0.005041 & Up \\
\hline OR4D11 & -1.307921 & 0.005046 & Down \\
\hline CAPRIN1 & 1.203686 & 0.005095 & Up \\
\hline PPP3R1 & 1.237317 & 0.005208 & Up \\
\hline PPIC & 1.224048 & 0.005238 & Up \\
\hline PRAMEF10 & -1.247763 & 0.005257 & Down \\
\hline MBTPS2 & -1.210053 & 0.00542 & Down \\
\hline UBTD2 & 1.203269 & 0.00543 & Up \\
\hline SEC11A & 1.208371 & 0.005449 & Up \\
\hline USP25 & 1.213603 & 0.005498 & Up \\
\hline PTMA & 1.217797 & 0.005513 & Up \\
\hline FKBP1C & 1.209098 & 0.005523 & Up \\
\hline ANKIB1 & 1.23614 & 0.005651 & Up \\
\hline GSPT1 & 1.233347 & 0.005715 & Up \\
\hline UCP2 & 1.209526 & 0.005735 & Up \\
\hline TGFB3 & 1.220573 & 0.005769 & Up \\
\hline HIST1H4G & 1.221559 & 0.005803 & Up \\
\hline IGLJ5 & -1.226509 & 0.005818 & Down \\
\hline
\end{tabular}


Table 1 (continued)

\begin{tabular}{|c|c|c|c|}
\hline Gene symbol & Fold change & p-value & Gene feature \\
\hline KIF5B & 1.306336 & 0.005897 & Up \\
\hline $\begin{array}{l}\text { TC10000913. } \\
\text { hg.1 }\end{array}$ & -1.233065 & 0.006202 & Down \\
\hline $\begin{array}{l}\text { TC03000927. } \\
\text { hg.1 }\end{array}$ & -1.285309 & 0.006207 & Down \\
\hline PGRMC1 & 1.230418 & 0.006286 & Up \\
\hline $\mathrm{HOXC5}$ & 1.236194 & 0.006291 & Up \\
\hline KRTAP13-4 & 1.202792 & 0.006315 & Up \\
\hline OR3A2 & -1.250913 & 0.006384 & Down \\
\hline SRSF6 & 1.240448 & 0.006389 & Up \\
\hline GTSF1L & -1.204976 & 0.006532 & Down \\
\hline SYNCRIP & 1.229937 & 0.006571 & Up \\
\hline HIST1H2BO & 1.347018 & 0.006699 & Up \\
\hline EPC2 & 1.212558 & 0.006748 & Up \\
\hline CTDSPL2 & 1.229495 & 0.006768 & Up \\
\hline OR10A3 & -1.206131 & 0.006817 & Down \\
\hline KRTAP7-1 & -1.297287 & 0.006896 & Down \\
\hline THOC2 & -1.220789 & 0.006979 & Down \\
\hline ETF1 & 1.225052 & 0.006984 & Up \\
\hline $\begin{array}{l}\text { TC11000974. } \\
\text { hg.1 }\end{array}$ & -1.273131 & 0.006994 & Down \\
\hline PRELID1 & 1.210411 & 0.007014 & Up \\
\hline IGHV3-38 & -1.267673 & 0.007029 & Down \\
\hline OR1L3 & -1.275722 & 0.007034 & Down \\
\hline SRP54 & 1.294575 & 0.007117 & Up \\
\hline EXOC5 & 1.217832 & 0.007122 & Up \\
\hline $\mathrm{OR} 2 \mathrm{H} 2$ & -1.263243 & 0.007292 & Down \\
\hline $\mathrm{OR} 2 \mathrm{H} 2$ & -1.263243 & 0.007292 & Down \\
\hline RNF122 & 1.27436 & 0.007373 & Up \\
\hline PTBP2 & -1.249936 & 0.007644 & Down \\
\hline ERP44 & 1.208521 & 0.007683 & Up \\
\hline CCR7 & -1.217568 & 0.007747 & Down \\
\hline QKI & 1.209051 & 0.008013 & Up \\
\hline ZFP42 & -1.215171 & 0.008141 & Down \\
\hline IGLV3-22 & -1.215548 & 0.008195 & Down \\
\hline DEFB103A & -1.213104 & 0.008451 & Down \\
\hline OR3A3 & -1.258226 & 0.008495 & Down \\
\hline TOP2B & 1.206659 & 0.008697 & Up \\
\hline PTEN & 1.221197 & 0.008785 & Up \\
\hline IGHV3-66 & -1.226136 & 0.008839 & Down \\
\hline PPP6C & 1.210188 & 0.008908 & Up \\
\hline PRAMEF8 & -1.238928 & 0.008923 & Down \\
\hline STRN3 & 1.291297 & 0.008948 & Up \\
\hline $\begin{array}{l}\text { TC01003841. } \\
\text { hg.1 }\end{array}$ & -1.354102 & 0.008997 & Down \\
\hline $\begin{array}{l}\text { TC05001047. } \\
\text { hg.1 }\end{array}$ & -1.208467 & 0.009046 & Down \\
\hline MAGT1 & 1.21942 & 0.00909 & Up \\
\hline $\mathrm{OR} 2 \mathrm{H} 2$ & -1.252699 & 0.009179 & Down \\
\hline $\mathrm{OR} 2 \mathrm{H} 2$ & -1.252699 & 0.009179 & Down \\
\hline $\mathrm{OR} 2 \mathrm{H} 2$ & -1.252699 & 0.009179 & Down \\
\hline
\end{tabular}

Table 1 (continued)

\begin{tabular}{|c|c|c|c|}
\hline Gene symbol & Fold change & $p$-value & Gene feature \\
\hline RPL4 & -1.201599 & 0.009336 & Down \\
\hline OSTC & 1.203947 & 0.009622 & Up \\
\hline IKZF5 & 1.216696 & 0.009868 & Up \\
\hline KRTAP10-11 & -1.205771 & 0.009912 & Down \\
\hline NLK & 1.209405 & 0.009932 & Up \\
\hline OR2W1 & 1.274304 & 0.009978 & Up \\
\hline OR2W1 & 1.274304 & 0.009978 & Up \\
\hline OR2W1 & 1.274304 & 0.009978 & Up \\
\hline OR2W1 & 1.274304 & 0.009978 & Up \\
\hline OR2W1 & 1.274304 & 0.009978 & Up \\
\hline OR2W1 & 1.274304 & 0.009978 & Up \\
\hline OR2W1 & 1.274304 & 0.009978 & Up \\
\hline OR2W1 & 1.274304 & 0.009978 & Up \\
\hline CD248 & 1.239436 & 0.010758 & Up \\
\hline HIST1H4F & 1.204154 & 0.010876 & Up \\
\hline OR6C1 & -1.216993 & 0.010911 & Down \\
\hline TMEM202 & -1.213705 & 0.011093 & Down \\
\hline TP53INP1 & -1.205295 & 0.011329 & Down \\
\hline KPNA3 & 1.21541 & 0.011511 & Up \\
\hline C7orf66 & -1.226557 & 0.011919 & Down \\
\hline TMSB4XP4 & 1.260484 & 0.011964 & Up \\
\hline TRAV24 & -1.229097 & 0.012215 & Down \\
\hline WDR26 & 1.201142 & 0.012239 & Up \\
\hline KRTAP9-3 & -1.229216 & 0.012293 & Down \\
\hline CSNK1A1L & 1.212513 & 0.012716 & Up \\
\hline $\begin{array}{l}\text { TC08001202. } \\
\text { hg.1 }\end{array}$ & -1.327872 & 0.013076 & Down \\
\hline TAS2R16 & -1.212834 & 0.013287 & Down \\
\hline IGHV1-8 & 1.229339 & 0.013312 & Up \\
\hline RAB2A & 1.248861 & 0.013563 & Up \\
\hline GPRIN2 & -1.234909 & 0.014257 & Down \\
\hline DEFB118 & -1.270079 & 0.014778 & Down \\
\hline $\begin{array}{l}\text { TC07000141. } \\
\text { hg.1 }\end{array}$ & -1.33934 & 0.015 & Down \\
\hline $\begin{array}{l}\text { TC02000056. } \\
\text { hg.1 }\end{array}$ & -1.236353 & 0.015231 & Down \\
\hline LCE1D & -1.234952 & 0.015432 & Down \\
\hline FAM218A & 1.265433 & 0.015487 & Up \\
\hline LCE2B & -1.208788 & 0.015511 & Down \\
\hline CRABP1 & -1.271706 & 0.015536 & Down \\
\hline OR1F1 & -1.231414 & 0.015841 & Down \\
\hline LOC100144595 & -1.207195 & 0.016023 & Down \\
\hline GALNT1 & 1.247945 & 0.016274 & Up \\
\hline LEP & -1.236716 & 0.016746 & Down \\
\hline DEFB136 & -1.203294 & 0.017223 & Down \\
\hline $\begin{array}{l}\text { TC07000218. } \\
\text { hg.1 }\end{array}$ & -1.256974 & 0.017302 & Down \\
\hline PPIAL4E & 1.263803 & 0.02144 & Up \\
\hline USP17 & 1.21594 & 0.026188 & Up \\
\hline
\end{tabular}


Table 1 (continued)

\begin{tabular}{|c|c|c|c|}
\hline Gene symbol & Fold change & $p$-value & Gene feature \\
\hline \multicolumn{4}{|c|}{$\begin{array}{l}\text { (C) Genes up/down-regulated in non-purified lung TCS }{ }^{\mathrm{V} 40} \text { compared with non- } \\
\text { purified primary lung TCS }\end{array}$} \\
\hline HSPA5 & 2.28735 & $5.20 \mathrm{E}-05$ & Up \\
\hline BNC1 & 1.464673 & $5.70 \mathrm{E}-05$ & Up \\
\hline CAPN6 & -1.711607 & $6.20 \mathrm{E}-05$ & Down \\
\hline HOXC6 & 1.334235 & $6.60 \mathrm{E}-05$ & Up \\
\hline COL12A1 & 1.349599 & $7.10 \mathrm{E}-05$ & Up \\
\hline IGFBP7 & 1.60287 & $7.60 E-05$ & Up \\
\hline GATA6 & 1.812957 & $8.10 \mathrm{E}-05$ & Up \\
\hline SLIT3 & 1.453596 & $8.60 \mathrm{E}-05$ & Up \\
\hline ANGPTL7 & 1.780209 & $9.10 \mathrm{E}-05$ & Up \\
\hline COL5A2 & 1.723719 & $9.60 \mathrm{E}-05$ & Up \\
\hline COL1A1 & 1.699369 & 0.000101 & Up \\
\hline PPARGC1A & -1.461747 & 0.000106 & Down \\
\hline MEIS2 & 1.482364 & 0.000111 & Up \\
\hline SYVN1 & 1.426147 & 0.000116 & Up \\
\hline MYH10 & 1.457796 & 0.000121 & Up \\
\hline MED12 & -1.423327 & 0.000126 & Down \\
\hline SMO & -1.306238 & 0.00013 & Down \\
\hline DNM1 & -1.263174 & 0.000135 & Down \\
\hline ERO1L & 1.270922 & 0.00014 & Up \\
\hline PBX1 & -1.307252 & 0.000145 & Down \\
\hline THBS1 & 1.469591 & 0.00015 & Up \\
\hline FBN1 & 1.535712 & 0.000155 & Up \\
\hline TRBV21OR9-2 & -1.373795 & 0.00016 & Down \\
\hline PTPRD & 1.295981 & 0.000165 & Up \\
\hline $\mathrm{BICC} 1$ & 1.467091 & 0.00017 & Up \\
\hline DUSP6 & 1.245629 & 0.000175 & Up \\
\hline TXNIP & 1.314454 & 0.00018 & Up \\
\hline FOXP2 & 1.294122 & 0.000185 & Up \\
\hline COL1A2 & 1.44837 & 0.00019 & Up \\
\hline ACTA1 & 1.287627 & 0.000195 & Up \\
\hline SEMA3A & -1.310532 & 0.000199 & Down \\
\hline DES & 1.335159 & 0.000204 & Up \\
\hline REXO2 & 1.380366 & 0.000209 & Up \\
\hline ACTN1 & 1.352297 & 0.000214 & Up \\
\hline VCL & 1.66324 & 0.000219 & Up \\
\hline PCDH19 & -1.617275 & 0.000224 & Down \\
\hline SLC6A8 & -1.239617 & 0.000229 & Down \\
\hline PCDH17 & -1.932881 & 0.000239 & Down \\
\hline OGT & -1.657267 & 0.000244 & Down \\
\hline HOXB5 & -1.442975 & 0.000249 & Down \\
\hline SEMA3D & 1.383364 & 0.000254 & Up \\
\hline MECOM & -1.327861 & 0.000259 & Down \\
\hline WT1 & 1.349575 & 0.000263 & Up \\
\hline KCNAB1 & 1.210026 & 0.000268 & Up \\
\hline RUNX1T1 & -1.360214 & 0.000273 & Down \\
\hline PLCE1 & 1.209156 & 0.000278 & Up \\
\hline COL3A1 & 1.499792 & 0.000283 & Up \\
\hline FAM102B & -1.353459 & 0.000288 & Down \\
\hline MBD6 & -1.351938 & 0.000293 & Down \\
\hline CRYBA1 & -1.269111 & 0.000298 & Down \\
\hline ANXA6 & 1.224881 & 0.000303 & Up \\
\hline
\end{tabular}

Table 1 (continued)

\begin{tabular}{|c|c|c|c|}
\hline Gene symbol & Fold change & p-value & Gene feature \\
\hline PCDH7 & 1.650618 & 0.000308 & Up \\
\hline PLXDC2 & 1.3089 & 0.000313 & Up \\
\hline FST & 1.553608 & 0.000318 & Up \\
\hline NUAK1 & 1.27549 & 0.000323 & Up \\
\hline COL4A5 & 1.276706 & 0.000327 & Up \\
\hline IGF1 & 1.254137 & 0.000332 & Up \\
\hline SEC23A & 1.389357 & 0.000337 & Up \\
\hline ATP1B1 & 1.276224 & 0.000342 & Up \\
\hline HS6ST2 & 1.230549 & 0.000347 & Up \\
\hline ZNF462 & 1.218491 & 0.000352 & Up \\
\hline FAM218A & 1.448595 & 0.000357 & Up \\
\hline SEC23B & 1.258266 & 0.000362 & Up \\
\hline PLOD2 & 1.25462 & 0.000367 & Up \\
\hline DNAJC1 & 1.413719 & 0.000372 & Up \\
\hline RAPH1 & 1.369576 & 0.000377 & Up \\
\hline GRIA3 & 1.691491 & 0.000382 & Up \\
\hline PSMC4 & 1.341278 & 0.000387 & Up \\
\hline SEC24A & 1.317102 & 0.000391 & Up \\
\hline TGFB1I1 & 1.214649 & 0.000396 & Up \\
\hline ELAVL2 & -1.263137 & 0.000401 & Down \\
\hline NRP2 & -1.307128 & 0.000406 & Down \\
\hline KIAA1199 & 1.371075 & 0.000411 & Up \\
\hline LPHN3 & -1.212789 & 0.000421 & Down \\
\hline ARRDC3 & -1.350686 & 0.000426 & Down \\
\hline KLHL13 & 1.212748 & 0.000431 & Up \\
\hline MYO1D & 1.385837 & 0.000436 & Up \\
\hline TM9SF3 & 1.307262 & 0.000441 & Up \\
\hline TFAP2A & -1.274332 & 0.000446 & Down \\
\hline ARL6IP1 & 1.244781 & 0.000451 & Up \\
\hline CPXM1 & 1.300338 & 0.000455 & Up \\
\hline CCND2 & -1.241703 & 0.00046 & Down \\
\hline SPTLC2 & 1.354194 & 0.000465 & Up \\
\hline NNAT & 1.392895 & 0.00047 & Up \\
\hline STK39 & 1.540497 & 0.000475 & Up \\
\hline ITGAV & 1.267551 & 0.00048 & Up \\
\hline LTBP1 & -1.280112 & 0.000485 & Down \\
\hline CELF4 & 1.232625 & 0.00049 & Up \\
\hline OSBP & 1.424925 & 0.000495 & Up \\
\hline MASP1 & -1.219414 & $5.00 E-04$ & Down \\
\hline FRMD5 & -1.36697 & 0.000505 & Down \\
\hline AP3S1 & 1.258065 & 0.00051 & Up \\
\hline BNC2 & -1.294519 & 0.000515 & Down \\
\hline TRPS1 & -1.34803 & 0.000519 & Down \\
\hline TUBG2 & 1.223767 & 0.000529 & Up \\
\hline CNN1 & 1.210395 & 0.000544 & Up \\
\hline TNC & 1.281125 & 0.000549 & Up \\
\hline GBF1 & 1.314328 & 0.000554 & Up \\
\hline NFAT5 & -1.519776 & 0.000559 & Down \\
\hline NFIA & 1.498716 & 0.000564 & Up \\
\hline ADAM22 & 1.202425 & 0.000584 & Up \\
\hline RBPJ & -1.246466 & 0.000588 & Down \\
\hline EGR1 & -1.356633 & 0.000593 & Down \\
\hline ILDR2 & 1.458561 & 0.000603 & Up \\
\hline
\end{tabular}


Table 1 (continued)

\begin{tabular}{|c|c|c|c|}
\hline Gene symbol & Fold change & p-value & Gene feature \\
\hline FSCN1 & 1.213951 & 0.000608 & Up \\
\hline VGLL3 & 1.201156 & 0.000623 & Up \\
\hline HIF1A & 1.377011 & 0.000633 & Up \\
\hline ATP2A2 & 1.25653 & 0.000638 & Up \\
\hline ARF6 & 1.256706 & 0.000648 & Up \\
\hline NETO1 & 1.206084 & 0.000662 & Up \\
\hline PLK1 & 1.239207 & 0.000667 & Up \\
\hline CAV1 & -1.227122 & 0.000682 & Down \\
\hline ATF6 & 1.278798 & 0.000697 & Up \\
\hline ATP1A1 & 1.260299 & 0.000702 & Up \\
\hline GPC3 & 1.252397 & 0.000707 & Up \\
\hline HSPA4 & 1.310919 & 0.000716 & Up \\
\hline MAP4K4 & 1.293954 & 0.000721 & Up \\
\hline $\mathrm{HOXC4}$ & 1.528822 & 0.000736 & Up \\
\hline JAG1 & 1.203931 & 0.000746 & Up \\
\hline INPPL1 & 1.248465 & 0.000756 & Up \\
\hline FOSB & -1.204723 & 0.000766 & Down \\
\hline KMT2D & -1.228557 & 0.000771 & Down \\
\hline PRPF19 & 1.227842 & 0.000776 & Up \\
\hline CCDC80 & 1.225889 & 0.00079 & Up \\
\hline TM9SF4 & 1.256719 & 0.000805 & Up \\
\hline NXN & 1.219999 & 0.00081 & Up \\
\hline COPB1 & 1.27883 & 0.00082 & Up \\
\hline SRPX & 1.337622 & 0.000825 & Up \\
\hline SMAD7 & 1.390506 & 0.00083 & Up \\
\hline VPS25 & 1.267452 & 0.000835 & Up \\
\hline TC18000270.hg.1 & 1.241506 & 0.000844 & Up \\
\hline DDX26B & -1.386832 & 0.000854 & Down \\
\hline SCD & 1.222675 & 0.000859 & Up \\
\hline KMT2A & -1.233077 & 0.000869 & Down \\
\hline JUNB & -1.289174 & 0.000874 & Down \\
\hline OR10P1 & -1.222678 & 0.000884 & Down \\
\hline LPCAT3 & 1.33701 & 0.000894 & Up \\
\hline ARHGAP1 & 1.390092 & 0.000909 & Up \\
\hline GRIA4 & 1.313269 & 0.000928 & Up \\
\hline GGCX & 1.210524 & 0.000948 & Up \\
\hline $\mathrm{CNIH}$ & 1.326625 & 0.000958 & Up \\
\hline SPARC & 1.31356 & 0.000963 & Up \\
\hline S100A2 & -1.206393 & 0.000968 & Down \\
\hline THBS2 & 1.201097 & 0.000973 & Up \\
\hline MEF2A & 1.231751 & 0.000977 & Up \\
\hline HOXC8 & 1.40158 & 0.000987 & Up \\
\hline SARS & -1.216959 & 0.001002 & Down \\
\hline HOXC4 & 1.205214 & 0.001017 & Up \\
\hline FEZ1 & -1.210057 & 0.001022 & Down \\
\hline HDGFRP3 & 1.360953 & 0.001027 & Up \\
\hline SMAD6 & 1.248102 & 0.001032 & Up \\
\hline EGR3 & -1.413218 & 0.001037 & Down \\
\hline $\mathrm{LPL}$ & 1.241889 & 0.001046 & Up \\
\hline CREB3L1 & 1.208352 & 0.001051 & Up \\
\hline EXT1 & 1.352789 & 0.001066 & Up \\
\hline SERPINH1 & 1.267578 & 0.001071 & Up \\
\hline $\mathrm{CDH} 11$ & 1.256532 & 0.001076 & Up \\
\hline
\end{tabular}

Table 1 (continued)

\begin{tabular}{|c|c|c|c|}
\hline Gene symbol & Fold change & p-value & Gene feature \\
\hline FAM126A & -1.231349 & 0.001086 & Down \\
\hline ZNF521 & -1.298692 & 0.00111 & Down \\
\hline PCDH18 & -1.284827 & 0.00113 & Down \\
\hline ACTN4 & 1.348564 & 0.00114 & Up \\
\hline C10orf53 & -1.240495 & 0.00115 & Down \\
\hline $\mathrm{EM} \times 2$ & 1.428467 & 0.001155 & Up \\
\hline TUBG1 & 1.240367 & 0.001169 & Up \\
\hline PLAG1 & -1.253787 & 0.001194 & Down \\
\hline NEO1 & 1.311703 & 0.001209 & Up \\
\hline $\mathrm{NR} 2 \mathrm{~F} 2$ & -1.327418 & 0.001214 & Down \\
\hline RAB39B & -1.208364 & 0.001229 & Down \\
\hline LCE1C & -1.227094 & 0.001234 & Down \\
\hline NPR2 & -1.277907 & 0.001243 & Down \\
\hline APH1A & 1.202275 & 0.001248 & Up \\
\hline PRR9 & -1.473668 & 0.001263 & Down \\
\hline FAM168A & 1.316093 & 0.001273 & Up \\
\hline LOX & 1.233141 & 0.001278 & Up \\
\hline ETV6 & 1.225393 & 0.001283 & Up \\
\hline MBNL1 & -1.348394 & 0.001312 & Down \\
\hline ZSWIM6 & 1.360747 & 0.001327 & Up \\
\hline GPM6B & -1.209186 & 0.001342 & Down \\
\hline FERMT2 & 1.204761 & 0.001347 & Up \\
\hline ISM1 & 1.222367 & 0.001357 & Up \\
\hline HECTD1 & 1.325901 & 0.001366 & Up \\
\hline GPC6 & 1.249832 & 0.001371 & Up \\
\hline SPRR1A & -1.304855 & 0.001376 & Down \\
\hline C17orf47 & -1.210708 & 0.001381 & Down \\
\hline POLA1 & -1.278784 & 0.001396 & Down \\
\hline RNF114 & 1.215485 & 0.001406 & Up \\
\hline PHLDB2 & 1.22064 & 0.001421 & Up \\
\hline BTG1 & -1.419463 & 0.001435 & Down \\
\hline $\mathrm{OR} 2 \mathrm{H} 2$ & -1.386141 & 0.00146 & Down \\
\hline RAB6A & 1.218989 & 0.00147 & Up \\
\hline STK19 & -1.226269 & 0.001482 & Down \\
\hline STK19 & -1.226269 & 0.001482 & Down \\
\hline EFNB1 & 1.214243 & 0.00149 & Up \\
\hline HIPK3 & 1.272379 & 0.001514 & Up \\
\hline $\mathrm{OR} 2 \mathrm{H} 2$ & -1.336272 & 0.001524 & Down \\
\hline OR4X2 & -1.231192 & 0.001539 & Down \\
\hline ZNF503 & 1.203437 & 0.001554 & Up \\
\hline$A B \mid 1$ & 1.207101 & 0.001568 & Up \\
\hline FAM188A & 1.354332 & 0.001578 & Up \\
\hline MBNL2 & 1.315575 & 0.001593 & Up \\
\hline $\mathrm{CDH} 2$ & 1.263605 & 0.001618 & Up \\
\hline FAM98A & 1.272083 & 0.001652 & Up \\
\hline DDB1 & 1.233239 & 0.001691 & Up \\
\hline TC11000975.hg.1 & -1.370641 & 0.001696 & Down \\
\hline DEFB128 & -1.229163 & 0.001701 & Down \\
\hline CYP51A1 & 1.215873 & 0.001706 & Up \\
\hline EFNA5 & 1.525255 & 0.001711 & Up \\
\hline CLINT1 & 1.352971 & 0.001721 & Up \\
\hline TCF4 & -1.350334 & 0.001736 & Down \\
\hline INTS5 & 1.272327 & 0.001741 & Up \\
\hline
\end{tabular}


Table 1 (continued)

\begin{tabular}{|c|c|c|c|}
\hline Gene symbol & Fold change & p-value & Gene feature \\
\hline GATM & 1.354506 & 0.00177 & Up \\
\hline GABRA3 & 1.32631 & 0.001775 & Up \\
\hline RAB1A & 1.472237 & 0.001785 & Up \\
\hline TC02001150.hg.1 & 1.202559 & 0.001795 & Up \\
\hline CAMK2N2 & 1.205549 & 0.0018 & Up \\
\hline HNRNPUL2 & 1.26108 & 0.001815 & Up \\
\hline FGF18 & 1.254064 & 0.001829 & Up \\
\hline LCE3B & -1.20559 & 0.001849 & Down \\
\hline SRPR & 1.282511 & 0.001859 & Up \\
\hline RNF138 & 1.200216 & 0.001864 & Up \\
\hline GABRB1 & 1.20311 & 0.001869 & Up \\
\hline $\mathrm{ZDHHC2}$ & 1.231848 & 0.001879 & Up \\
\hline PTGS2 & -1.235944 & 0.001888 & Down \\
\hline ANGPT1 & -1.239367 & 0.001923 & Down \\
\hline CDC27 & 1.301846 & 0.001933 & Up \\
\hline ZFP36L2 & -1.309184 & 0.001957 & Down \\
\hline THOC2 & -1.298345 & 0.001972 & Down \\
\hline AMMECR1L & 1.204913 & 0.001982 & Up \\
\hline TAGLN & 1.21811 & 0.001997 & Up \\
\hline SPRR1B & -1.386398 & 0.002007 & Down \\
\hline ADAMTS6 & -1.251192 & 0.002021 & Down \\
\hline LCE3E & -1.238425 & 0.002036 & Down \\
\hline TEAD1 & 1.316471 & 0.00209 & Up \\
\hline OR4D11 & -1.397758 & 0.002115 & Down \\
\hline PSMD14 & 1.269457 & 0.002164 & Up \\
\hline INSL6 & 1.241962 & 0.002169 & Up \\
\hline FBXO33 & 1.240947 & 0.002174 & Up \\
\hline $\mathrm{OR} 2 \mathrm{H} 2$ & -1.335922 & 0.002211 & Down \\
\hline $\mathrm{OR} 2 \mathrm{H} 2$ & -1.335922 & 0.002211 & Down \\
\hline LCE1D & -1.24485 & 0.002233 & Down \\
\hline STIM1 & 1.291974 & 0.002253 & Up \\
\hline TRBV6-8 & -1.383088 & 0.002297 & Down \\
\hline PARVA & 1.212507 & 0.002307 & Up \\
\hline TC11000974.hg.1 & -1.355331 & 0.002322 & Down \\
\hline CTGF & 1.278251 & 0.002327 & Up \\
\hline PRKG1 & 1.245661 & 0.002341 & Up \\
\hline TLN1 & 1.209426 & 0.002356 & Up \\
\hline OR3A2 & -1.258635 & 0.002361 & Down \\
\hline NPR3 & 1.252907 & 0.002366 & Up \\
\hline CYGB & 1.286596 & 0.002376 & Up \\
\hline ID1 & 1.302257 & 0.002396 & Up \\
\hline HSPA2 & 1.291744 & 0.002401 & Up \\
\hline DERL2 & 1.338707 & 0.002415 & Up \\
\hline $\mathrm{OR} 2 \mathrm{H} 2$ & -1.331198 & 0.002469 & Down \\
\hline $\mathrm{OR} 2 \mathrm{H} 2$ & -1.331198 & 0.002469 & Down \\
\hline $\mathrm{OR} 2 \mathrm{H} 2$ & -1.331198 & 0.002469 & Down \\
\hline EIF4G2 & 1.351151 & 0.002548 & Up \\
\hline PAPOLA & 1.311535 & 0.002578 & Up \\
\hline PPP3R1 & 1.259841 & 0.002588 & Up \\
\hline PDIA3 & 1.321177 & 0.002597 & Up \\
\hline HSP90B1 & 1.297674 & 0.002627 & Up \\
\hline EIF4A1 & 1.248301 & 0.002642 & Up \\
\hline TNPO3 & -1.20247 & 0.002676 & Down \\
\hline
\end{tabular}

Table 1 (continued)

\begin{tabular}{|c|c|c|c|}
\hline Gene symbol & Fold change & p-value & Gene feature \\
\hline UCP2 & 1.245612 & 0.002686 & Up \\
\hline EEF1G & 1.228255 & 0.00274 & Up \\
\hline TC13000014.hg.1 & -1.237888 & 0.002745 & Down \\
\hline TC02002617.hg.1 & -1.519734 & 0.00275 & Down \\
\hline PFN1 & 1.248818 & 0.00276 & Up \\
\hline ERLEC1 & 1.219854 & 0.00277 & Up \\
\hline GSPT1 & 1.296726 & 0.002878 & Up \\
\hline TC15001723.hg.1 & -1.244628 & 0.002883 & Down \\
\hline C8orf49 & -1.225571 & 0.002888 & Down \\
\hline STAT3 & 1.204904 & 0.002957 & Up \\
\hline MAN1A1 & 1.22109 & 0.002982 & Up \\
\hline ACTR1A & 1.210577 & 0.003046 & Up \\
\hline TAF10 & 1.234009 & 0.003075 & Up \\
\hline ONECUT2 & -1.203425 & 0.0031 & Down \\
\hline PPP2R5E & 1.303497 & 0.003105 & Up \\
\hline STT3A & 1.268152 & 0.003115 & Up \\
\hline TC03001641.hg.1 & -1.243194 & 0.003134 & Down \\
\hline TGFB3 & 1.241756 & 0.003159 & Up \\
\hline DICER1 & 1.208135 & 0.003164 & Up \\
\hline LCE2A & -1.356188 & 0.003213 & Down \\
\hline HIST1H4J & 1.203634 & 0.003257 & Up \\
\hline P4HA1 & 1.268543 & 0.003277 & Up \\
\hline TCF20 & -1.200225 & 0.003287 & Down \\
\hline $\mathrm{HOXC5}$ & 1.25077 & 0.003302 & Up \\
\hline MTX2 & 1.203709 & 0.003316 & Up \\
\hline MUT & -1.206951 & 0.003376 & Down \\
\hline CPD & 1.217165 & 0.003405 & Up \\
\hline PRELP & -1.216805 & 0.00343 & Down \\
\hline OR10D3 & -1.289554 & 0.003558 & Down \\
\hline EFNB2 & 1.322603 & 0.003563 & Up \\
\hline MBTPS2 & -1.219406 & 0.003577 & Down \\
\hline ACVR2A & 1.277435 & 0.003632 & Up \\
\hline PCDH10 & 1.289149 & 0.003641 & Up \\
\hline OR3A3 & -1.294084 & 0.003676 & Down \\
\hline VAT1L & 1.224017 & 0.003681 & Up \\
\hline ANKIB1 & 1.250405 & 0.003705 & Up \\
\hline ATP6AP2 & -1.203066 & 0.003829 & Down \\
\hline TMSB10 & 1.33115 & 0.003893 & Up \\
\hline MYL9 & 1.238522 & 0.003976 & Up \\
\hline $\mathrm{P} 4 \mathrm{HA} 3$ & 1.223749 & 0.004006 & Up \\
\hline LOC 100144595 & -1.210771 & 0.004045 & Down \\
\hline CCDC47 & 1.254626 & 0.004085 & Up \\
\hline PTP4A1 & 1.207393 & 0.004104 & Up \\
\hline SNX12 & -1.231653 & 0.004163 & Down \\
\hline TC03000927.hg.1 & -1.34441 & 0.004173 & Down \\
\hline PRAMEF10 & -1.226358 & 0.004218 & Down \\
\hline HIST1H2BF & 1.201242 & 0.004331 & Up \\
\hline HIST1H4F & 1.278307 & 0.004355 & Up \\
\hline LSM2 & -1.23126 & 0.00436 & Down \\
\hline PSMC6 & 1.28439 & 0.00437 & Up \\
\hline TMEM57 & 1.214675 & 0.00438 & Up \\
\hline TRGV8 & -1.24305 & 0.004449 & Down \\
\hline SEC11A & 1.212858 & 0.004508 & Up \\
\hline
\end{tabular}


Table 1 (continued)

\begin{tabular}{|c|c|c|c|}
\hline Gene symbol & Fold change & $p$-value & Gene feature \\
\hline OR51B6 & -1.25219 & 0.004528 & Down \\
\hline ZNF830 & 1.223738 & 0.004552 & Up \\
\hline TMEM238 & 1.259086 & 0.004582 & Up \\
\hline TOP1 & 1.207832 & 0.004685 & Up \\
\hline LELP1 & -1.254685 & 0.004764 & Down \\
\hline MMD & 1.342077 & 0.004774 & Up \\
\hline LCE1A & -1.207411 & 0.004804 & Down \\
\hline IGKV1D-13 & -1.253243 & 0.004808 & Down \\
\hline FZD8 & 1.20833 & 0.004853 & Up \\
\hline OR5A2 & -1.583233 & 0.004887 & Down \\
\hline C1QL3 & 1.202082 & 0.005015 & Up \\
\hline PANK3 & 1.209123 & 0.00506 & Up \\
\hline KIF5B & 1.306357 & 0.005094 & Up \\
\hline SRP54 & 1.335205 & 0.005153 & Up \\
\hline ETF1 & 1.247056 & 0.005385 & Up \\
\hline HIST1H4G & 1.221559 & 0.005399 & Up \\
\hline TRIP12 & 1.250366 & 0.005463 & Up \\
\hline TRAJ59 & 1.280347 & 0.005591 & Up \\
\hline DEFB133 & -1.217544 & 0.00568 & Down \\
\hline HIST1H2BH & 1.324812 & 0.005739 & Up \\
\hline ACTL8 & -1.34524 & 0.005828 & Down \\
\hline HIST1H2BO & 1.331009 & 0.005867 & Up \\
\hline C16orf87 & 1.227019 & 0.005877 & Up \\
\hline HIST1H1B & 1.236488 & 0.005971 & Up \\
\hline PPP3CA & 1.2093 & 0.006 & Up \\
\hline DAZAP2 & -1.246936 & 0.006069 & Down \\
\hline C7orf66 & -1.257301 & 0.006168 & Down \\
\hline IRF2BPL & 1.222737 & 0.006197 & Up \\
\hline USP12 & 1.245254 & 0.006202 & Up \\
\hline PGAP2 & -1.236121 & 0.006217 & Down \\
\hline WNK1 & -1.317909 & 0.006315 & Down \\
\hline FAR1 & 1.239903 & 0.006424 & Up \\
\hline HOXA2 & 1.321485 & 0.006497 & Up \\
\hline IGHV3-48 & -1.200801 & 0.006537 & Down \\
\hline LCE2C & -1.207171 & 0.006714 & Down \\
\hline PPIC & 1.208975 & 0.006822 & Up \\
\hline COPA & 1.213652 & 0.006847 & Up \\
\hline SPANXN3 & 1.276398 & 0.006916 & Up \\
\hline DCUN1D5 & 1.204299 & 0.006931 & Up \\
\hline RAP1B & 1.238418 & 0.006946 & Up \\
\hline CMAS & 1.312695 & 0.007064 & Up \\
\hline NLK & 1.232188 & 0.007211 & Up \\
\hline HIST1H2BE & 1.335735 & 0.007216 & Up \\
\hline HRNR & -1.31544 & 0.007221 & Down \\
\hline OR4A16 & 1.208665 & 0.007285 & Up \\
\hline C8orf48 & 1.252595 & 0.007477 & Up \\
\hline CSNK1A1 & 1.214353 & 0.007497 & Up \\
\hline UBE2M & 1.219256 & 0.00761 & Up \\
\hline ZNF281 & 1.250794 & 0.007817 & Up \\
\hline IGHD2-21 & -1.332138 & 0.007837 & Down \\
\hline E2F3 & 1.206829 & 0.008019 & Up \\
\hline TC10000913.hg.1 & -1.226511 & 0.008108 & Down \\
\hline NCKAP1 & 1.22608 & 0.008285 & Up \\
\hline
\end{tabular}

Table 1 (continued)

\begin{tabular}{|c|c|c|c|}
\hline Gene symbol & Fold change & p-value & Gene feature \\
\hline PPP6C & 1.215357 & 0.008364 & Up \\
\hline SEC62 & 1.25965 & 0.008536 & Up \\
\hline EXOC5 & 1.207899 & 0.00862 & Up \\
\hline FKBP1C & 1.20189 & 0.008787 & Up \\
\hline ZBTB6 & 1.223895 & 0.008964 & Up \\
\hline TRBV6-7 & -1.261291 & 0.009048 & Down \\
\hline DEFB136 & -1.297342 & 0.009122 & Down \\
\hline FGF10 & -1.247986 & 0.009186 & Down \\
\hline OR4C6 & 1.293432 & 0.00927 & Up \\
\hline GFPT1 & 1.20605 & 0.009309 & Up \\
\hline PSMA6 & 1.216218 & 0.009353 & Up \\
\hline TC01002282.hg.1 & -1.202154 & 0.009477 & Down \\
\hline KRTAP9-8 & -1.222393 & 0.009486 & Down \\
\hline LCE2B & -1.251225 & 0.009595 & Down \\
\hline TC17000686.hg.1 & -1.224599 & 0.009846 & Down \\
\hline TMSB4XP4 & 1.276305 & 0.009935 & Up \\
\hline LOC100509638 & -1.228863 & 0.010215 & Down \\
\hline OR51L1 & -1.209919 & 0.010486 & Down \\
\hline TC07000141.hg.1 & -1.389386 & 0.011299 & Down \\
\hline USP17 & 1.231161 & 0.011338 & Up \\
\hline BMPR1A & 1.225957 & 0.011535 & Up \\
\hline IGHV1OR21-1 & -1.207255 & 0.011811 & Down \\
\hline LOC728819 & 1.209199 & 0.011968 & Up \\
\hline WDR26 & 1.21391 & 0.012145 & Up \\
\hline TC15001075.hg.1 & -1.206125 & 0.012165 & Down \\
\hline TRAPPC1 & 1.207842 & 0.012264 & Up \\
\hline STRN3 & 1.251923 & 0.012589 & Up \\
\hline IGKV1D-37 & 1.290804 & 0.012667 & Up \\
\hline TC05000944.hg.1 & -1.209012 & 0.012855 & Down \\
\hline KRTAP6-3 & -1.209466 & 0.013017 & Down \\
\hline TC01001567.hg.1 & -1.220919 & 0.013106 & Down \\
\hline TC08000204.hg.1 & -1.257586 & 0.013199 & Down \\
\hline TRAV24 & -1.224177 & 0.013224 & Down \\
\hline KPNA3 & 1.208042 & 0.013244 & Up \\
\hline CALHM1 & -1.281085 & 0.01346 & Down \\
\hline KRTAP5-6 & -1.213396 & 0.013514 & Down \\
\hline OR5W2 & -1.227139 & 0.013682 & Down \\
\hline $\mathrm{HIST} 2 \mathrm{H} 2 \mathrm{BE}$ & 1.203613 & 0.013928 & Up \\
\hline TAOK1 & 1.203131 & 0.014223 & Up \\
\hline RBMXL2 & 1.242693 & 0.014371 & Up \\
\hline MGC15705 & 1.248494 & 0.014499 & Up \\
\hline NOP58 & 1.210344 & 0.014898 & Up \\
\hline TC01003841.hg.1 & -1.272609 & 0.014947 & Down \\
\hline CASK & -1.202663 & 0.015371 & Down \\
\hline GPX5 & -1.231507 & 0.015435 & Down \\
\hline IGL6 & -1.209384 & 0.015651 & Down \\
\hline OR6C1 & -1.220835 & 0.015715 & Down \\
\hline OR1L3 & -1.26804 & 0.015981 & Down \\
\hline IGKV1-9 & -1.205738 & 0.016425 & Down \\
\hline CSNK1A1L & 1.236726 & 0.016981 & Up \\
\hline OR51A4 & -1.251899 & 0.017158 & Down \\
\hline TC07000847.hg.1 & 1.200866 & 0.017404 & Up \\
\hline TAS2R16 & -1.220859 & 0.017424 & Down \\
\hline
\end{tabular}


Table 1 (continued)

\begin{tabular}{|c|c|c|c|}
\hline Gene symbol & Fold change & $p$-value & Gene feature \\
\hline EIF2S2 & 1.202591 & 0.018104 & Up \\
\hline RAB2A & 1.218567 & 0.018571 & Up \\
\hline OR56A5 & 1.204074 & 0.022471 & Up \\
\hline Gene symbol & Fold change & $p$-value & Gene feature \\
\hline \multicolumn{4}{|c|}{$\begin{array}{l}\text { (D) Genes up/down-regulated in purified TCS }{ }^{\text {SV40 }} \text { compared with non-purified lung } \\
T C S^{S V 40}\end{array}$} \\
\hline ZBTB18 & -1.554972 & 0.000298 & Down \\
\hline MID2 & -1.541494 & 0.000126 & Down \\
\hline HSPA5 & -1.518909 & 0.000465 & Down \\
\hline TSC22D2 & -1.5017 & 0.00048 & Down \\
\hline $\mathrm{VCL}$ & -1.495358 & 0.000204 & Down \\
\hline MEIS2 & -1.460638 & $8.60 \mathrm{E}-05$ & Down \\
\hline STK39 & -1.423681 & 0.000313 & Down \\
\hline CTGF & -1.421299 & 0.000268 & Down \\
\hline DOCK11 & -1.415118 & 0.000426 & Down \\
\hline ATF6 & -1.409588 & 0.000199 & Down \\
\hline SLC7A11 & -1.404048 & 0.000101 & Down \\
\hline BRAF & -1.396899 & 0.000755 & Down \\
\hline NLK & -1.384761 & 0.00076 & Down \\
\hline ERO1L & -1.366172 & $5.20 \mathrm{E}-05$ & Down \\
\hline HIST1H2BE & -1.360048 & 0.003083 & Down \\
\hline MEF2A & -1.354049 & 0.000135 & Down \\
\hline CELF1 & -1.34869 & 0.000706 & Down \\
\hline HPRT1 & -1.348486 & 0.001415 & Down \\
\hline TBPL1 & -1.344601 & 0.000573 & Down \\
\hline FAM188A & -1.344027 & 0.000652 & Down \\
\hline AZIN1 & -1.322403 & 0.000288 & Down \\
\hline SYVN1 & -1.309011 & 0.000145 & Down \\
\hline HIST1H2BO & -1.308399 & 0.002104 & Down \\
\hline STRBP & -1.307068 & 0.000362 & Down \\
\hline ABCB7 & -1.302327 & 0.00239 & Down \\
\hline EFNB2 & -1.298157 & 0.002606 & Down \\
\hline ANKRD1 & -1.293984 & 0.000455 & Down \\
\hline SLC6A9 & -1.292011 & 0.000244 & Down \\
\hline RND1 & -1.288509 & 0.000258 & Down \\
\hline $\mathrm{HOXC4}$ & -1.286961 & 0.001459 & Down \\
\hline PGRMC1 & -1.285024 & 0.000775 & Down \\
\hline IGHV1-8 & -1.283244 & 0.003162 & Down \\
\hline GSPT1 & -1.277447 & 0.002286 & Down \\
\hline FN1 & -1.272748 & 0.001287 & Down \\
\hline AKT3 & -1.269844 & 0.000436 & Down \\
\hline STIM1 & -1.269255 & 0.00139 & Down \\
\hline EFNA5 & -1.268694 & 0.002818 & Down \\
\hline CCDC47 & -1.265588 & 0.001528 & Down \\
\hline DES & -1.265153 & 0.00013 & Down \\
\hline PDZD8 & -1.258982 & 0.000696 & Down \\
\hline GATA6 & -1.253813 & 0.000637 & Down \\
\hline SEL1L & -1.25119 & 0.000603 & Down \\
\hline HDGFRP3 & -1.249545 & 0.000947 & Down \\
\hline ZNF827 & -1.249169 & 0.000175 & Down \\
\hline NEO1 & -1.24814 & 0.001996 & Down \\
\hline MYH10 & -1.247444 & 0.00049 & Down \\
\hline
\end{tabular}

Table 1 (continued)

\begin{tabular}{|c|c|c|c|}
\hline Gene symbol & Fold change & p-value & Gene feature \\
\hline C16orf87 & -1.247099 & 0.002311 & Down \\
\hline HES1 & -1.244124 & 0.000308 & Down \\
\hline HERPUD1 & -1.239348 & 0.000229 & Down \\
\hline DUSP6 & -1.237186 & 0.002138 & Down \\
\hline RAPH1 & -1.235005 & 0.000992 & Down \\
\hline CDC42BPA & -1.233364 & 0.00203 & Down \\
\hline ACTN4 & -1.232684 & 0.00174 & Down \\
\hline ZNF462 & -1.232013 & 0.000111 & Down \\
\hline VPRBP & -1.230287 & 0.00078 & Down \\
\hline RALGAPA1 & -1.229069 & 0.001804 & Down \\
\hline UPRT & -1.229057 & 0.002852 & Down \\
\hline UBE2W & -1.228348 & 0.000785 & Down \\
\hline SAMD4B & -1.227886 & 0.000667 & Down \\
\hline MTRNR2L4 & -1.224725 & 0.00172 & Down \\
\hline SEC24A & -1.224506 & 0.000421 & Down \\
\hline AMMECR1L & -1.222428 & 0.00047 & Down \\
\hline PRKAA2 & -1.221814 & 0.003029 & Down \\
\hline ACTN1 & -1.218558 & 0.000623 & Down \\
\hline OSBP & -1.217315 & 0.001464 & Down \\
\hline VLDLR & -1.216913 & 0.000239 & Down \\
\hline MSI2 & -1.211676 & 0.000731 & Down \\
\hline MSN & -1.209973 & 0.002842 & Down \\
\hline LRRC58 & -1.209927 & 0.000273 & Down \\
\hline COL4A5 & -1.209539 & 0.000317 & Down \\
\hline SLAIN2 & -1.207964 & 0.002094 & Down \\
\hline NDFIP1 & -1.206718 & 0.001932 & Down \\
\hline PPP2R5B & -1.20442 & 0.001134 & Down \\
\hline VPS25 & -1.20254 & 0.00079 & Down \\
\hline CPD & -1.201293 & 0.002626 & Down \\
\hline COLEC12 & 1.200894 & 0.00173 & Up \\
\hline THBS3 & 1.201895 & 0.000726 & Up \\
\hline KITLG & 1.202163 & 0.000337 & Up \\
\hline SETBP1 & 1.202182 & 0.000765 & Up \\
\hline LOXL1 & 1.202879 & 0.001203 & Up \\
\hline PRKD1 & 1.20361 & $9.10 E-05$ & Up \\
\hline ZFP36L1 & 1.203909 & 0.001755 & Up \\
\hline EDDM3B & 1.205244 & 0.001159 & Up \\
\hline ANGPT1 & 1.207484 & 0.001346 & Up \\
\hline MMP2 & 1.208515 & 0.000165 & Up \\
\hline FAM170B & 1.210029 & 0.000923 & Up \\
\hline LELP1 & 1.213262 & 0.002473 & Up \\
\hline IGFBP4 & 1.215597 & 0.002011 & Up \\
\hline STC1 & 1.217133 & 0.000928 & Up \\
\hline EPHA7 & 1.218188 & 0.000568 & Up \\
\hline PRDM6 & 1.219262 & 0.000618 & Up \\
\hline ACTRT1 & 1.219685 & 0.001509 & Up \\
\hline $\mathrm{XDH}$ & 1.222067 & 0.000209 & Up \\
\hline OR4D11 & 1.224206 & 0.000874 & Up \\
\hline HOXA11 & 1.22653 & 0.002001 & Up \\
\hline PAK3 & 1.228895 & 0.000381 & Up \\
\hline $\mathrm{BNC2}$ & 1.242835 & 0.000303 & Up \\
\hline SLC16A2 & 1.248637 & 0.000593 & Up \\
\hline NRN1 & 1.251496 & 0.000564 & Up \\
\hline
\end{tabular}


Table 1 (continued)

\begin{tabular}{|c|c|c|c|}
\hline Gene symbol & Fold change & $p$-value & Gene feature \\
\hline GDPD2 & 1.251704 & 0.000189 & Up \\
\hline RUNX1T1 & 1.251964 & 0.000352 & Up \\
\hline PCDH18 & 1.252101 & 0.000263 & Up \\
\hline PCDH9 & 1.255458 & 0.001848 & Up \\
\hline MMP16 & 1.25842 & 0.000155 & Up \\
\hline PRRX1 & 1.259453 & 0.001144 & Up \\
\hline LRP1 & 1.263799 & 0.000997 & Up \\
\hline SEMA5A & 1.267548 & $7.10 \mathrm{E}-05$ & Up \\
\hline PBX1 & 1.270348 & 0.000185 & Up \\
\hline $\mathrm{RDH} 10$ & 1.271163 & $6.60 \mathrm{E}-05$ & Up \\
\hline VCAN & 1.277158 & 0.000121 & Up \\
\hline HOXB8 & 1.284619 & 0.00016 & Up \\
\hline FOXG1 & 1.287872 & 0.001824 & Up \\
\hline MT1G & 1.289954 & 0.000485 & Up \\
\hline SOCS3 & 1.291472 & 0.002252 & Up \\
\hline TBX4 & 1.309269 & 0.00017 & Up \\
\hline ADAMTS15 & 1.313557 & 0.000278 & Up \\
\hline FGF7 & 1.320284 & 0.000805 & Up \\
\hline HOXA5 & 1.322982 & 0.00144 & Up \\
\hline MME & 1.331844 & 0.00015 & Up \\
\hline $\mathrm{DBC} 1$ & 1.332864 & $8.10 E-05$ & Up \\
\hline ZEB2 & 1.333152 & 0.000249 & Up \\
\hline LTBP1 & 1.335297 & 0.00018 & Up \\
\hline BTG1 & 1.338416 & 0.00112 & Up \\
\hline MT1H & 1.346673 & 0.000327 & Up \\
\hline UGCG & 1.347608 & $9.60 \mathrm{E}-05$ & Up \\
\hline TCF4 & 1.350209 & 0.000559 & Up \\
\hline MEIS1 & 1.358188 & 0.000445 & Up \\
\hline BHLHE22 & 1.358601 & 0.00014 & Up \\
\hline MT1B & 1.367849 & 0.000539 & Up \\
\hline TSHZ1 & 1.396499 & 0.000106 & Up \\
\hline FGF10 & 1.434899 & $5.00 \mathrm{E}-04$ & Up \\
\hline ARRDC3 & 1.438034 & 0.000194 & Up \\
\hline FIGN & 1.458516 & 0.000253 & Up \\
\hline NR2F2 & 1.464969 & 0.000549 & Up \\
\hline ZNF521 & 1.487048 & 0.000283 & Up \\
\hline GPM6B & 1.570568 & $7.60 \mathrm{E}-05$ & Up \\
\hline HOXB5 & 1.727273 & $5.70 \mathrm{E}-05$ & Up \\
\hline FRMD5 & 1.736738 & $6.20 \mathrm{E}-05$ & Up \\
\hline SFRP2 & 1.894966 & 0.000116 & Up \\
\hline Gene symbol & Fold change & $p$-value & Gene feature \\
\hline \multicolumn{4}{|c|}{$\begin{array}{l}\text { (E) Genes up/down-regulated in purified } T C S^{S V 40} \text { compared with purified primary lung } \\
\text { TCS }\end{array}$} \\
\hline COL3A1 & 2.033893 & $5.20 \mathrm{E}-05$ & Up \\
\hline FST & 1.763096 & $5.70 \mathrm{E}-05$ & Up \\
\hline SLIT3 & 1.476233 & $6.20 E-05$ & Up \\
\hline COL1A1 & 1.754216 & $6.60 E-05$ & Up \\
\hline FRMD5 & 1.529829 & $7.10 E-05$ & Up \\
\hline FBN1 & 1.662323 & $7.60 E-05$ & Up \\
\hline ZFP36L1 & 1.546307 & $8.10 E-05$ & Up \\
\hline TNC & 1.410268 & $8.60 E-05$ & Up \\
\hline $\mathrm{PCDH7}$ & 1.75739 & $9.10 \mathrm{E}-05$ & Up \\
\hline
\end{tabular}

Table 1 (continued)

\begin{tabular}{|c|c|c|c|}
\hline Gene symbol & Fold change & $p$-value & Gene feature \\
\hline $\mathrm{DBC1}$ & 1.550832 & $9.60 \mathrm{E}-05$ & Up \\
\hline MMP2 & 1.365924 & 0.000101 & Up \\
\hline NNAT & 1.470003 & 0.000106 & Up \\
\hline STAT3 & 1.55145 & 0.000111 & Up \\
\hline KLHDC10 & -1.299299 & 0.000116 & Down \\
\hline KIAA1199 & 1.437971 & 0.000121 & Up \\
\hline BHLHE22 & 1.541751 & 0.000126 & Up \\
\hline SPRY2 & 1.451741 & 0.00013 & Up \\
\hline LOXL1 & 1.602346 & 0.000135 & Up \\
\hline TSHZ1 & 1.635298 & 0.00014 & Up \\
\hline COL5A2 & 1.534133 & 0.000145 & Up \\
\hline HOXA2 & 1.907531 & 0.00015 & Up \\
\hline FGF10 & 1.384277 & 0.000155 & Up \\
\hline COL12A1 & 1.291331 & 0.00016 & Up \\
\hline ZNF503 & 1.448549 & 0.000165 & Up \\
\hline NID1 & 1.254112 & 0.00017 & Up \\
\hline NFIX & 1.50204 & 0.000175 & Up \\
\hline ANGPTL7 & 1.516574 & 0.00018 & Up \\
\hline COL1A2 & 1.516627 & 0.000185 & Up \\
\hline |GFBP7 & 1.527419 & 0.000189 & Up \\
\hline MEIS1 & 1.661917 & 0.000194 & Up \\
\hline ILDR2 & 1.354674 & 0.000199 & Up \\
\hline IER5L & 1.407578 & 0.000204 & Up \\
\hline HMGCS1 & 1.325093 & 0.000209 & Up \\
\hline CALM3 & 1.256977 & 0.000214 & Up \\
\hline CELF2 & 1.661174 & 0.000219 & Up \\
\hline PCDH10 & 1.601334 & 0.000224 & Up \\
\hline NDST1 & 1.200203 & 0.000229 & Up \\
\hline CPXM1 & 1.387893 & 0.000234 & Up \\
\hline PPARGC1A & -1.43313 & 0.000239 & Down \\
\hline ZEB2 & 1.389745 & 0.000244 & Up \\
\hline SPRR1B & -1.417133 & 0.000249 & Down \\
\hline MMP16 & 1.351496 & 0.000253 & Up \\
\hline RNF150 & 1.301929 & 0.000258 & Up \\
\hline LOX & 1.275704 & 0.000263 & Up \\
\hline CBX6 & 1.291217 & 0.000273 & Up \\
\hline ZNF521 & 1.642922 & 0.000278 & Up \\
\hline MME & 1.32368 & 0.000283 & Up \\
\hline GDPD2 & 1.247925 & 0.000288 & Up \\
\hline FMR1 & 1.627765 & 0.000293 & Up \\
\hline PDGFRA & 1.21296 & 0.000298 & Up \\
\hline C3orf58 & 1.227127 & 0.000303 & Up \\
\hline SFRP2 & 2.026877 & 0.000308 & Up \\
\hline FBLN2 & 1.287435 & 0.000313 & Up \\
\hline CYP51A1 & 1.229665 & 0.000317 & Up \\
\hline ARF6 & 1.234812 & 0.000322 & Up \\
\hline RBPJ & -1.345805 & 0.000327 & Down \\
\hline GPM6B & 1.420512 & 0.000332 & Up \\
\hline NFIA & 1.616514 & 0.000337 & Up \\
\hline MBD6 & -1.277385 & 0.000342 & Down \\
\hline EXT1 & 1.446781 & 0.000347 & Up \\
\hline MID2 & -1.366576 & 0.000352 & Down \\
\hline
\end{tabular}


Table 1 (continued)

\begin{tabular}{|c|c|c|c|}
\hline Gene symbol & Fold change & $p$-value & Gene feature \\
\hline LRP6 & 1.5391 & 0.000357 & Up \\
\hline BICC1 & 1.448877 & 0.000362 & Up \\
\hline WT1 & 1.215831 & 0.000367 & Up \\
\hline CHD3 & 1.226874 & 0.000372 & Up \\
\hline THBS1 & 1.572993 & 0.000377 & Up \\
\hline UPRT & -1.413812 & 0.000381 & Down \\
\hline NLGN2 & 1.224543 & 0.000386 & Up \\
\hline NXN & 1.274044 & 0.000391 & Up \\
\hline MED12 & -1.307768 & 0.000396 & Down \\
\hline LRP1 & 1.46219 & 0.000401 & Up \\
\hline HOXB5 & 1.333689 & 0.000406 & Up \\
\hline KLF7 & 1.264161 & 0.000411 & Up \\
\hline ACLY & 1.361581 & 0.000416 & Up \\
\hline $\mathrm{RDH} 10$ & 1.355596 & 0.000421 & Up \\
\hline DPYSL2 & 1.315251 & 0.000431 & Up \\
\hline PRRX1 & 1.553947 & 0.000436 & Up \\
\hline LPHN2 & 1.258686 & 0.00044 & Up \\
\hline GATA6 & 1.43309 & 0.000445 & Up \\
\hline $\mathrm{CDH} 11$ & 1.32449 & 0.00045 & Up \\
\hline PRG4 & 1.243068 & 0.000455 & Up \\
\hline PCDH17 & -1.543509 & 0.00046 & Down \\
\hline NFIB & 1.600764 & 0.000465 & Up \\
\hline VCAN & 1.21944 & 0.00047 & Up \\
\hline FAM98A & 1.298444 & 0.000475 & Up \\
\hline EFEMP1 & 1.226158 & 0.00048 & Up \\
\hline FOXP2 & 1.205803 & 0.000485 & Up \\
\hline PCDHGC5 & 1.331799 & $5.00 \mathrm{E}-04$ & Up \\
\hline THBS2 & 1.266764 & 0.000504 & Up \\
\hline HIF1A & 1.440204 & 0.000509 & Up \\
\hline IGFBP4 & 1.30241 & 0.000514 & Up \\
\hline MARK1 & 1.233597 & 0.000519 & Up \\
\hline KMT2D & -1.200632 & 0.000524 & Down \\
\hline SCD & 1.32132 & 0.000534 & Up \\
\hline LPCAT3 & 1.43367 & 0.000539 & Up \\
\hline TBPL1 & -1.393924 & 0.000544 & Down \\
\hline PDE1A & 1.230559 & 0.000554 & Up \\
\hline GRIA3 & 1.359144 & 0.000564 & Up \\
\hline EFEMP2 & 1.222385 & 0.000568 & Up \\
\hline TCF20 & -1.318507 & 0.000573 & Down \\
\hline PDE7B & 1.556907 & 0.000578 & Up \\
\hline HTRA1 & -1.270642 & 0.000583 & Down \\
\hline HOXA5 & 1.58066 & 0.000593 & Up \\
\hline CYB5R1 & -1.258022 & 0.000603 & Down \\
\hline SETBP1 & 1.318692 & 0.000608 & Up \\
\hline CACNAIC & 1.265093 & 0.000613 & Up \\
\hline ADAMTS2 & 1.224627 & 0.000618 & Up \\
\hline DOCK9 & -1.215588 & 0.000623 & Down \\
\hline OGT & -1.564357 & 0.000632 & Down \\
\hline COLEC12 & 1.376494 & 0.000637 & Up \\
\hline ZBTB18 & -1.778186 & 0.000642 & Down \\
\hline KITLG & 1.25933 & 0.000647 & Up \\
\hline GFPT2 & 1.276106 & 0.000652 & Up \\
\hline
\end{tabular}

Table 1 (continued)

\begin{tabular}{|c|c|c|c|}
\hline Gene symbol & Fold change & $p$-value & Gene feature \\
\hline GABRA3 & 1.435938 & 0.000657 & Up \\
\hline SLC16A2 & 1.320833 & 0.000662 & Up \\
\hline PCDHB1 & -1.242627 & 0.000667 & Down \\
\hline EPHA7 & 1.315288 & 0.000677 & Up \\
\hline SLCO3A1 & 1.220403 & 0.000682 & Up \\
\hline DDX26B & -1.214763 & 0.000687 & Down \\
\hline SEMA3D & 1.235461 & 0.000692 & Up \\
\hline ADAMTS15 & 1.389205 & 0.000696 & Up \\
\hline MAN1A1 & 1.226513 & 0.000701 & Up \\
\hline TCF7L2 & 1.319781 & 0.000706 & Up \\
\hline PBX3 & 1.27527 & 0.000711 & Up \\
\hline SLIT2 & 1.356085 & 0.000721 & Up \\
\hline TENM4 & -1.247945 & 0.000731 & Down \\
\hline PARP6 & -1.252561 & 0.000736 & Down \\
\hline KLF9 & 1.320073 & 0.000755 & Up \\
\hline IGF1 & 1.441883 & 0.00076 & Up \\
\hline HOXB8 & 1.273005 & 0.00077 & Up \\
\hline ARID5B & 1.28496 & 0.000775 & Up \\
\hline PKM & 1.322278 & 0.00078 & Up \\
\hline BMP1 & 1.215932 & 0.000785 & Up \\
\hline PCDH19 & -1.416186 & 0.000795 & Down \\
\hline FEZ1 & -1.257686 & $8.00 E-04$ & Down \\
\hline NPR2 & -1.26446 & 0.00081 & Down \\
\hline STRBP & -1.352471 & 0.000819 & Down \\
\hline DNAJC1 & 1.241378 & 0.000824 & Up \\
\hline WNK1 & -1.574397 & 0.000829 & Down \\
\hline DHCR24 & 1.236232 & 0.000844 & Up \\
\hline EGR1 & -1.325378 & 0.000849 & Down \\
\hline MT1M & 1.25661 & 0.000864 & Up \\
\hline AKAP8L & -1.20015 & 0.000874 & Down \\
\hline ATP1B1 & 1.211897 & 0.000879 & Up \\
\hline GATM & 1.291347 & 0.000883 & Up \\
\hline TRBV6-8 & -1.35926 & 0.000893 & Down \\
\hline FZD8 & 1.649274 & 0.000903 & Up \\
\hline AHCYL2 & -1.206907 & 0.000913 & Down \\
\hline PPP3CA & 1.344731 & 0.000918 & Up \\
\hline SURF4 & 1.211797 & 0.000923 & Up \\
\hline CCND2 & -1.224408 & 0.000938 & Down \\
\hline RAB11B & 1.26997 & 0.000943 & Up \\
\hline FHL1 & 1.202305 & 0.000952 & Up \\
\hline TOX & 1.397482 & 0.000957 & Up \\
\hline FOXC1 & 1.242409 & 0.000967 & Up \\
\hline AIDA & 1.274415 & 0.000977 & Up \\
\hline TNFAIP1 & 1.202941 & 0.000982 & Up \\
\hline THBS3 & 1.214669 & 0.000987 & Up \\
\hline CLMP & 1.294055 & 0.000992 & Up \\
\hline DOCK3 & -1.231871 & 0.001002 & Down \\
\hline DDIT3 & -1.40635 & 0.001006 & Down \\
\hline GPC6 & 1.248637 & 0.001011 & Up \\
\hline $\mathrm{ABCB} 7$ & -1.307951 & 0.001016 & Down \\
\hline ETV6 & 1.353185 & 0.001026 & Up \\
\hline CHKA & -1.240302 & 0.001051 & Down \\
\hline
\end{tabular}


Table 1 (continued)

\begin{tabular}{|c|c|c|c|}
\hline Gene symbol & Fold change & p-value & Gene feature \\
\hline R3HDM2 & -1.252354 & 0.001061 & Down \\
\hline TLN1 & 1.226184 & 0.001085 & Up \\
\hline PFN1 & 1.394161 & 0.00109 & Up \\
\hline ADAMTS4 & 1.218343 & 0.001095 & Up \\
\hline CLIP3 & 1.213372 & 0.0011 & Up \\
\hline ILK & 1.304537 & 0.00111 & Up \\
\hline PLXDC2 & 1.301745 & 0.00113 & Up \\
\hline PABPC4 & -1.218394 & 0.001134 & Down \\
\hline $\mathrm{NR} 2 \mathrm{~F} 2$ & 1.485732 & 0.001174 & Up \\
\hline PPAP2B & 1.278773 & 0.001189 & Up \\
\hline FIGN & 1.399002 & 0.001198 & Up \\
\hline SPARC & 1.228554 & 0.001223 & Up \\
\hline AKT3 & -1.215306 & 0.001233 & Down \\
\hline TC10000913.hg.1 & -1.44934 & 0.001238 & Down \\
\hline RHOT1 & -1.311667 & 0.001243 & Down \\
\hline NUAK1 & 1.245725 & 0.001253 & Up \\
\hline SOCS3 & 1.418689 & 0.001287 & Up \\
\hline TC02002617.hg.1 & -1.617442 & 0.001292 & Down \\
\hline TC07000141.hg.1 & -1.394869 & 0.001302 & Down \\
\hline MYO1D & 1.296444 & 0.001307 & Up \\
\hline $\mathrm{CDH} 2$ & 1.216156 & 0.001317 & Up \\
\hline TCF4 & 1.260214 & 0.001321 & Up \\
\hline FUBP1 & -1.41599 & 0.001326 & Down \\
\hline ARRDC3 & 1.309616 & 0.001341 & Up \\
\hline CYGB & 1.229195 & 0.001351 & Up \\
\hline TBX4 & 1.238548 & 0.001356 & Up \\
\hline MUT & -1.208493 & 0.001361 & Down \\
\hline MT1H & 1.423387 & 0.001371 & Up \\
\hline MBTPS2 & -1.36196 & 0.001381 & Down \\
\hline RAB1B & 1.221867 & 0.00143 & Up \\
\hline AZIN1 & -1.289217 & 0.001435 & Down \\
\hline CNTFR & 1.224893 & 0.001445 & Up \\
\hline NRN1 & 1.247028 & 0.001449 & Up \\
\hline PRPF19 & 1.221176 & 0.001469 & Up \\
\hline HPRT1 & -1.432868 & 0.001484 & Down \\
\hline PARVA & 1.248515 & 0.001494 & Up \\
\hline MSN & -1.307225 & 0.001499 & Down \\
\hline SPRR2F & -1.204892 & 0.001509 & Down \\
\hline DOCK11 & -1.249159 & 0.001513 & Down \\
\hline $\mathrm{H} 3 \mathrm{~F} 3 \mathrm{~A}$ & 1.288991 & 0.001518 & Up \\
\hline MAP4K3 & 1.215421 & 0.001528 & Up \\
\hline RGL1 & 1.23898 & 0.001543 & Up \\
\hline PSAT1 & -1.279406 & 0.001572 & Down \\
\hline $\mathrm{BNC1}$ & 1.24342 & 0.001617 & Up \\
\hline HNRNPUL2 & 1.206099 & 0.001622 & Up \\
\hline MSI2 & -1.235799 & 0.001627 & Down \\
\hline FAM71B & 1.244138 & 0.001656 & Up \\
\hline SEC23A & 1.252503 & 0.001661 & Up \\
\hline CNOT6L & -1.344862 & 0.001666 & Down \\
\hline CTDSP2 & 1.310633 & 0.001676 & Up \\
\hline FGF7 & 1.282848 & 0.001691 & Up \\
\hline MYH10 & 1.216826 & 0.001696 & Up \\
\hline
\end{tabular}

Table 1 (continued)

\begin{tabular}{|c|c|c|c|}
\hline Gene symbol & Fold change & p-value & Gene feature \\
\hline EMP3 & 1.28252 & 0.00171 & Up \\
\hline SOX4 & 1.304636 & 0.00172 & Up \\
\hline NR4A3 & 1.23027 & 0.00175 & Up \\
\hline USP17 & 1.224951 & 0.00176 & Up \\
\hline LDB1 & 1.225476 & 0.001764 & Up \\
\hline ATF6 & -1.284147 & 0.001789 & Down \\
\hline $\mathrm{ABI} 1$ & 1.224035 & 0.001804 & Up \\
\hline $\mathrm{P} 4 \mathrm{HA} 3$ & 1.256545 & 0.001814 & Up \\
\hline PTEN & 1.427111 & 0.001838 & Up \\
\hline ССТ2 & -1.25044 & 0.001843 & Down \\
\hline KMT2A & -1.254142 & 0.001848 & Down \\
\hline ISM1 & 1.407921 & 0.001873 & Up \\
\hline DEFB113 & -1.306901 & 0.001878 & Down \\
\hline EFNA5 & 1.216599 & 0.001942 & Up \\
\hline RBP1 & 1.223532 & 0.002006 & Up \\
\hline HSPA9 & -1.22658 & 0.002015 & Down \\
\hline TRBV21OR9-2 & -1.275752 & 0.002035 & Down \\
\hline JHDM1D & -1.22599 & 0.002089 & Down \\
\hline PRKAA2 & -1.288077 & 0.002109 & Down \\
\hline GPC3 & 1.209065 & 0.002129 & Up \\
\hline INTS5 & 1.218441 & 0.002158 & Up \\
\hline EPT1 & -1.215737 & 0.002163 & Down \\
\hline PAK3 & 1.209358 & 0.002178 & Up \\
\hline SARS & -1.377121 & 0.002183 & Down \\
\hline MYL9 & 1.215861 & 0.002202 & Up \\
\hline CALR & 1.328144 & 0.002217 & Up \\
\hline PLK1 & 1.234092 & 0.002222 & Up \\
\hline PDIA3 & 1.320796 & 0.002247 & Up \\
\hline C14orf1 & 1.234254 & 0.002266 & Up \\
\hline PRELID1 & 1.300632 & 0.002281 & Up \\
\hline TC01001567.hg.1 & -1.230659 & 0.002301 & Down \\
\hline SULF1 & 1.211791 & 0.002326 & Up \\
\hline CPSF6 & -1.256973 & 0.00234 & Down \\
\hline NR4A2 & 1.213443 & 0.002345 & Up \\
\hline NR1D1 & -1.219622 & 0.00235 & Down \\
\hline RAB5C & 1.222135 & 0.002375 & Up \\
\hline REXO2 & 1.290263 & 0.002399 & Up \\
\hline LMO4 & 1.200112 & 0.002409 & Up \\
\hline GPR174 & 1.216642 & 0.002414 & Up \\
\hline AP2S1 & 1.217261 & 0.002419 & Up \\
\hline DYNLL2 & 1.251735 & 0.002449 & Up \\
\hline HUWE1 & -1.260641 & 0.002468 & Down \\
\hline VPRBP & -1.26699 & 0.002473 & Down \\
\hline BMPR1A & 1.33189 & 0.002478 & Up \\
\hline $\mathrm{DIO} 3$ & 1.203031 & 0.002493 & Up \\
\hline TM9SF4 & 1.216029 & 0.002547 & Up \\
\hline RAB1A & 1.410474 & 0.002572 & Up \\
\hline CEBPB & 1.214692 & 0.002577 & Up \\
\hline ACTR1A & 1.216125 & 0.002606 & Up \\
\hline LOC100509638 & -1.209083 & 0.002611 & Down \\
\hline CHMP4B & 1.224858 & 0.002636 & Up \\
\hline ARHGAP1 & 1.325099 & 0.00265 & Up \\
\hline
\end{tabular}


Table 1 (continued)

\begin{tabular}{|c|c|c|c|}
\hline Gene symbol & Fold change & p-value & Gene feature \\
\hline HMGA1 & -1.240781 & 0.002655 & Down \\
\hline TOP1 & 1.272427 & 0.00267 & Up \\
\hline EGR3 & -1.295132 & 0.002675 & Down \\
\hline C9orf172 & 1.228614 & 0.002685 & Up \\
\hline ID3 & 1.245858 & 0.002695 & Up \\
\hline CD248 & 1.388851 & 0.0027 & Up \\
\hline FAM168B & 1.234437 & 0.002768 & Up \\
\hline RCN3 & 1.226194 & 0.002783 & Up \\
\hline MT1A & 1.288399 & 0.002798 & Up \\
\hline EIF4B & -1.207714 & 0.002803 & Down \\
\hline GTPBP2 & -1.208847 & 0.002813 & Down \\
\hline TMSB10 & 1.386714 & 0.002847 & Up \\
\hline GABARAP & 1.284343 & 0.002852 & Up \\
\hline IGHV3-38 & -1.236566 & 0.002916 & Down \\
\hline TC1 1000974.hg.1 & -1.267039 & 0.002931 & Down \\
\hline RAB39B & -1.258083 & 0.002985 & Down \\
\hline MMP14 & 1.257854 & 0.003034 & Up \\
\hline NFAT5 & -1.270263 & 0.003069 & Down \\
\hline LCE3D & -1.208922 & 0.003093 & Down \\
\hline TMTC2 & 1.248958 & 0.003108 & Up \\
\hline USP12 & 1.408625 & 0.003133 & Up \\
\hline ANKRD1 & -1.219365 & 0.003152 & Down \\
\hline RBBP5 & -1.210453 & 0.003172 & Down \\
\hline PTMA & 1.251584 & 0.003197 & Up \\
\hline ACTA1 & 1.225395 & 0.003246 & Up \\
\hline RNF141 & -1.205359 & 0.00333 & Down \\
\hline FGFBP1 & -1.200483 & 0.003364 & Down \\
\hline RPL4 & -1.217294 & 0.003408 & Down \\
\hline CASK & -1.354502 & 0.003413 & Down \\
\hline LCE3E & -1.213158 & 0.003453 & Down \\
\hline $\mathrm{EPC2}$ & 1.261066 & 0.003467 & Up \\
\hline NOXRED1 & -1.234805 & 0.003507 & Down \\
\hline MT1B & 1.263775 & 0.003517 & Up \\
\hline CCR7 & -1.260932 & 0.003561 & Down \\
\hline BRAF & -1.278428 & 0.003595 & Down \\
\hline RPS27 & -1.246149 & 0.0036 & Down \\
\hline HNRNPH2 & 1.328316 & 0.00361 & Up \\
\hline IGHV2-26 & -1.203819 & 0.00364 & Down \\
\hline DEFB115 & -1.226413 & 0.003679 & Down \\
\hline PTBP2 & -1.331489 & 0.003763 & Down \\
\hline RPS6KB1 & 1.216347 & 0.003896 & Up \\
\hline MT2A & 1.290212 & 0.003935 & Up \\
\hline TARDBP & -1.205193 & 0.003969 & Down \\
\hline SYT11 & 1.24811 & 0.004014 & Up \\
\hline MT1F & 1.263703 & 0.004024 & Up \\
\hline SFRP1 & 1.245684 & 0.004053 & Up \\
\hline SEMA3A & -1.202169 & 0.004073 & Down \\
\hline TC14000204.hg.1 & 1.20508 & 0.004122 & Up \\
\hline TSC22D2 & -1.302317 & 0.004127 & Down \\
\hline KCNJ2 & 1.282504 & 0.004176 & Up \\
\hline SPTLC2 & 1.242016 & 0.004309 & Up \\
\hline POLA1 & -1.263183 & 0.004373 & Down \\
\hline
\end{tabular}

Table 1 (continued)

\begin{tabular}{|c|c|c|c|}
\hline Gene symbol & Fold change & p-value & Gene feature \\
\hline HIPK3 & 1.207585 & 0.004398 & Up \\
\hline HIST2H2AA4 & 1.242261 & 0.004407 & Up \\
\hline TEAD1 & 1.201058 & 0.004432 & Up \\
\hline FBXO33 & 1.217546 & 0.004471 & Up \\
\hline ENOX2 & -1.227983 & 0.00453 & Down \\
\hline OR5A2 & -1.31152 & 0.00455 & Down \\
\hline THOC2 & -1.305497 & 0.004555 & Down \\
\hline CAPN6 & -1.245443 & 0.004614 & Down \\
\hline ZNF281 & 1.297079 & 0.004624 & Up \\
\hline NFKBIA & 1.21647 & 0.004649 & Up \\
\hline TBC1D19 & -1.208949 & 0.004683 & Down \\
\hline TC03000927.hg.1 & -1.21805 & 0.004688 & Down \\
\hline EDDM3B & 1.206252 & 0.004693 & Up \\
\hline TC19000375.hg.1 & -1.233615 & 0.004703 & Down \\
\hline OR5AU1 & -1.225907 & 0.004752 & Down \\
\hline PSMA3 & 1.210788 & 0.004781 & Up \\
\hline PABPC1 & -1.24424 & 0.004796 & Down \\
\hline OR2AJ1 & -1.209642 & 0.004988 & Down \\
\hline PPIAL4A & 1.303974 & 0.004993 & Up \\
\hline FIGF & 1.222296 & 0.005082 & Up \\
\hline TP53INP1 & -1.227022 & 0.00519 & Down \\
\hline DNAJA2 & 1.20171 & 0.005215 & Up \\
\hline SAP30 & 1.223176 & 0.005254 & Up \\
\hline OR4F21 & -1.206284 & 0.005461 & Down \\
\hline OR1L3 & -1.211827 & 0.005485 & Down \\
\hline TBX15 & 1.209874 & 0.005638 & Up \\
\hline IPO7 & -1.287169 & 0.005751 & Down \\
\hline ACVR2A & 1.227166 & 0.005889 & Up \\
\hline FOXG1 & 1.300571 & 0.005918 & Up \\
\hline AGFG1 & 1.205554 & 0.006032 & Up \\
\hline MURC & -1.204566 & 0.006169 & Down \\
\hline OR10D3 & -1.304006 & 0.006337 & Down \\
\hline ADAMTS5 & 1.212932 & 0.006342 & Up \\
\hline EID3 & 1.207392 & 0.007065 & Up \\
\hline MT1G & 1.209678 & 0.007784 & Up \\
\hline MSANTD2 & -1.200157 & 0.007966 & Down \\
\hline KDM6A & -1.246053 & 0.007971 & Down \\
\hline DERL2 & 1.224813 & 0.008443 & Up \\
\hline HSPA5 & 1.242949 & 0.008561 & Up \\
\hline POM121L2 & -1.202141 & 0.009423 & Down \\
\hline CCIN & 1.410994 & 0.009492 & Up \\
\hline TC11000975.hg.1 & -1.23782 & 0.009501 & Down \\
\hline SYNCRIP & 1.202486 & 0.009526 & Up \\
\hline OR1F1 & -1.200102 & 0.009708 & Down \\
\hline TRAM1L1 & 1.227933 & 0.009723 & Up \\
\hline PPIAL4C & 1.292198 & 0.009954 & Up \\
\hline TC11000959.hg.1 & -1.206482 & 0.010584 & Down \\
\hline KRTAP29-1 & -1.332407 & 0.010889 & Down \\
\hline TC07000218.hg.1 & -1.236963 & 0.011884 & Down \\
\hline ALX1 & -1.216059 & 0.012036 & Down \\
\hline HEXIM1 & 1.213559 & 0.012292 & Up \\
\hline OR10J5 & -1.31215 & 0.012991 & Down \\
\hline
\end{tabular}


Table 1 (continued)

\begin{tabular}{lcll}
\hline Gene symbol & Fold change & p-value & Gene feature \\
\hline IGLJ4 & 1.339919 & 0.014029 & Up \\
C10orf85 & 1.259314 & 0.014064 & Up \\
RNF122 & 1.213118 & 0.014384 & Up \\
PPIAL4C & 1.217453 & 0.015481 & Up \\
\hline Gene symbol & p-value & q-value & Gene feature
\end{tabular}

(F) Genes up/down-regulated in purified primary lung TCs compared with nonpurified primary lung TCS

\begin{tabular}{llll} 
ZNF521 & $5.20 \mathrm{E}-05$ & 0 & Down \\
NFIX & $6.20 \mathrm{E}-05$ & 0 & Down \\
ZFP36L2 & 0.000263 & 0.038653 & Down \\
NR2F2 & 0.00013 & 0 & Down \\
SLIT2 & $7.60 \mathrm{E}-05$ & 0 & Down \\
NFIB & 0.000155 & 0 & Down \\
NFAT5 & 0.000288 & 0.038653 & Down \\
LRP6 & $5.70 E-05$ & 0 & Down \\
FAM102B & 0.00019 & 0 & Down \\
TCF4 & 0.000372 & 0.045591 & Down \\
HES1 & 0.000406 & 0.045591 & Down \\
PFN1 & 0.000387 & 0.045591 & Down \\
TSHZ1 & $5.00 E-04$ & 0.04939 & Down \\
SPRY2 & 0.000268 & 0.038653 & Down \\
CTGF & $9.10 E-05$ & 0 & Down \\
ARRDC3 & 0.000244 & 0.038653 & Down \\
LOXL1 & 0.000682 & 0.04939 & Down \\
NLGN2 & 0.000116 & 0 & Down \\
ZEB2 & 0.00017 & 0 & Down \\
CTDSP2 & 0.00051 & 0.04939 & Down \\
FBLN5 & 0.000293 & 0.038653 & Down \\
FRMD5 & 0.000342 & 0.045591 & Down \\
RGL1 & 0.000416 & 0.045591 & Down \\
\hline & & &
\end{tabular}

$\mathrm{TCs}^{\mathrm{SV} 40}$ provides a repeatable and stable cell tool for deep investigation of biological roles.

SV40-infection or transformation of lung TCs alters gene expression profiles of cells at certain degrees. SV40 as a polyomavirus with icosahedral capsids of $45 \mathrm{~nm}$ and a $5.25 \mathrm{~kb}$-long circular double-stranded DNA can replicate in macaques as its natural host, leading to chronic asymptomatic infections. SV40 small and large $\mathrm{T}$ antigen was transduced using lentivirus to immortalize primary cells [18]. SV40 has been strongly considered as a clinical candidate of gene delivery, replacement, or therapy, due to the lack of immunogenicity in humans and capacity to induce immune tolerance to transgene proteins. Toscano et al. [19] used the current SV40 vector genome to generate Verobased packaging cell vector particles and expresses a large amount of the SV40 large T antigen. It indicates that SV40 delivery can be an approach or alternative of clinical gene therapy. In the present study, mouse lung TCs were transduced and immortalized with a sequence of SV40 small and large T antigen in lentivirus. Excepted for increased capability of cell proliferation, we found that morphology, measured biomarkers, movement, and ultrastructure of $\mathrm{TCs}^{\mathrm{SV} 40}$ are the same as primary TCs directly harvested from lungs. However, we did find a number of gene expression in $\mathrm{TCs}^{\mathrm{SV} 40}$ were different from primary TCs, although the meaning and values of altered gene expression profiles in $\mathrm{TCs}^{\mathrm{SV} 40}$ remain unclear.

There are numbers of questions and considerations on biological behaviors of cells with gene editing, especially about the long-term side-effects and pathophysiological responses of gene-edited cells to challenges. Although the aim of the present study to establish a mouse lung telocyte cell-line for deeply understanding molecular mechanisms of TCs, it should be aware the regulation and translational ethics of preclinical activities for the genome editing [20]. Gene-edited TCs in the present study do not need to perform the large-scale cross-platform comparisons of safety and specificity of those edited TCs as discussed for the potential of clinical applications [21], while we noticed a clear off-target effects of mouse lung TCs on the survival time and passage of TCs. The second passage of $\mathrm{TCs}^{\mathrm{SV} 40}$ had the strongest capacity of cell proliferation, while such capacity declined with the increase of telocyte passage. We found the characterizations of phenomes and functions of $\mathrm{TCs}^{\mathrm{SV} 40}$ were more stable between consecutive passages 5 and 30. Our finding is similar to the report from Taciak et al. [22] that the phenotype and functional stability of the RAW 264.7 cell line were evaluated from the 5th to 50th passage and suggested the RAW 264.7 cell line could remain stable 
Table 2 Summary of LncRNA expressed preferentially in primary TCs, SV40-transformed TCs, primary lung cells, and SV40-transformed primary lung cells

\begin{tabular}{|c|c|c|c|c|c|c|}
\hline Compared pairs & $U p>0$ & $U p>2$ & $U p>4$ & Down $>0$ & Down $>2$ & Down $>4$ \\
\hline $\begin{array}{l}\text { Purified TCs }{ }^{\text {Sv40 }} \\
\text { vs. non-purified } \\
\text { primary lung TCS }\end{array}$ & 77 & 3 & 0 & 579 & 16 & 2 \\
\hline $\begin{array}{l}\text { Non-purified } \\
\text { lung TCs }{ }^{\text {SV } 40} \text { vs. } \\
\text { purified primary } \\
\text { lung TCs }\end{array}$ & 306 & 15 & 4 & 756 & 15 & 0 \\
\hline $\begin{array}{l}\text { Non-purified lung } \\
\text { TCs }{ }^{\text {SV40 }} \text { vs. non- } \\
\text { purified primary } \\
\text { lung TCs }\end{array}$ & 114 & 7 & 2 & 607 & 20 & 0 \\
\hline $\begin{array}{l}\text { Purified TCs }{ }^{\text {sv40 }} \\
\text { vs. non-purified } \\
\text { lung TCs } s^{\text {Sv40 }}\end{array}$ & 7 & 0 & 0 & 41 & 4 & 1 \\
\hline $\begin{array}{l}\text { Purified TCs } \mathrm{SV}^{\mathrm{V} 40} \mathrm{vs} \text {. } \\
\text { purified primary } \\
\text { lung TCs }\end{array}$ & 112 & 11 & 0 & 499 & 12 & 1 \\
\hline $\begin{array}{l}\text { Purified primary } \\
\text { lung TCs vs. non- } \\
\text { purified primary } \\
\text { lung TCs }\end{array}$ & 0 & 0 & 0 & 13 & 2 & 1 \\
\hline
\end{tabular}

\begin{tabular}{llll}
\hline Probe set ID & Fold change & p-value & Gene feature \\
\hline (A) Genes up/down-regulated in SV40-transformed TCs compared with \\
non-purified primary lung TCs & & \\
TC17000728.hg.1 & -3.568496 & $2.70 \mathrm{E}-05$ & Down \\
TC06001978.hg.1 & -2.084397 & $3.00 \mathrm{E}-05$ & Down \\
TC08000302.hg.1 & -1.909385 & $3.30 \mathrm{E}-05$ & Down \\
TC07001784.hg.1 & -1.941664 & $3.50 \mathrm{E}-05$ & Down \\
TC03003114.hg.1 & 2.957271 & $3.80 \mathrm{E}-05$ & Up \\
TC11002382.hg.1 & -5.521638 & $4.00 \mathrm{E}-05$ & Down \\
TC09000963.hg.1 & -2.006316 & $4.30 \mathrm{E}-05$ & Down \\
TC15000452.hg.1 & -2.008375 & $4.60 \mathrm{E}-05$ & Down \\
TC16001289.hg.1 & -1.75485 & $4.80 \mathrm{E}-05$ & Down \\
TC02000396.hg.1 & -1.817337 & $5.10 \mathrm{E}-05$ & Down \\
TC06000639.hg.1 & -2.1972 & $5.40 \mathrm{E}-05$ & Down \\
TC12000927.hg.1 & -2.098063 & $5.60 \mathrm{E}-05$ & Down \\
TC08002253.hg.1 & -3.102457 & $5.90 \mathrm{E}-05$ & Down \\
TC11001010.hg.1 & -1.660071 & $6.10 \mathrm{E}-05$ & Down \\
TC17001801.hg.1 & -2.429971 & $6.40 \mathrm{E}-05$ & Down \\
TC02001602.hg.1 & -1.575753 & $6.70 \mathrm{E}-05$ & Down \\
TC02001940.hg.1 & -1.965258 & $6.90 \mathrm{E}-05$ & Down \\
TC07000780.hg.1 & -2.054482 & $7.20 \mathrm{E}-05$ & Down \\
TC19000767.hg.1 & -1.795968 & $7.40 \mathrm{E}-05$ & Down \\
TC09001648.hg.1 & -2.05885 & $7.70 \mathrm{E}-05$ & Down \\
TC05001593.hg.1 & -1.786234 & $8.00 \mathrm{E}-05$ & Down \\
TC09000971.hg.1 & -1.864315 & $8.20 \mathrm{E}-05$ & Down \\
TC04000160.hg.1 & -1.653172 & $8.50 \mathrm{E}-05$ & Down \\
TC0X001624.hg.1 & -1.966802 & $8.70 \mathrm{E}-05$ & Down \\
TC10002919.hg.1 & -1.570089 & $9.00 \mathrm{E}-05$ & Down \\
TC03002042.hg.1 & -1.634325 & $9.30 \mathrm{E}-05$ & Down \\
TC08001415.hg.1 & -1.709011 & $9.50 \mathrm{E}-05$ & Down \\
TC02000336.hg.1 & -1.6597 & $9.80 \mathrm{E}-05$ & Down \\
\hline
\end{tabular}

Table 2 (continued)

\begin{tabular}{|c|c|c|c|}
\hline Probe set ID & Fold change & p-value & Gene feature \\
\hline TC01006068.hg.1 & -1.773582 & 0.000101 & Down \\
\hline TC1 1000967.hg.1 & -2.600896 & 0.000103 & Down \\
\hline TC20001277.hg.1 & 1.567066 & 0.000106 & Up \\
\hline TC19000951.hg.1 & -1.643102 & 0.000108 & Down \\
\hline TC05000152.hg.1 & -1.698079 & 0.000111 & Down \\
\hline TC12000188.hg.1 & -1.705076 & 0.000114 & Down \\
\hline TC02000205.hg.1 & -1.840391 & 0.000116 & Down \\
\hline TC02002456.hg.1 & -1.803182 & 0.000119 & Down \\
\hline TC06002006.hg.1 & -1.642511 & 0.000121 & Down \\
\hline TC01003510.hg.1 & -1.59482 & 0.000124 & Down \\
\hline TC08001864.hg.1 & 1.822 & 0.000127 & Up \\
\hline TC20000952.hg.1 & -1.813744 & 0.000129 & Down \\
\hline TC04002890.hg.1 & 1.584375 & 0.000132 & Up \\
\hline TC1 1002894.hg.1 & 1.642096 & 0.000135 & Up \\
\hline TC03001841.hg.1 & -1.694023 & 0.000137 & Down \\
\hline TC04001635.hg.1 & -2.085812 & 0.00014 & Down \\
\hline TC04002560.hg.1 & 2.11556 & 0.000142 & Up \\
\hline TC16001498.hg.1 & 1.522853 & 0.000145 & Up \\
\hline TC15001604.hg.1 & -1.523167 & 0.000148 & Down \\
\hline TC20000373.hg.1 & -1.641514 & 0.00015 & Down \\
\hline TC12000633.hg.1 & -1.690876 & 0.000153 & Down \\
\hline TC13000783.hg.1 & -2.460055 & 0.000155 & Down \\
\hline TC15001326.hg.1 & -1.512893 & 0.000158 & Down \\
\hline TC12001286.hg.1 & -1.794425 & 0.000161 & Down \\
\hline TC01005824.hg.1 & -1.623728 & 0.000163 & Down \\
\hline TC19001567.hg.1 & 1.574923 & 0.000166 & Up \\
\hline TC17001773.hg.1 & -1.52185 & 0.000168 & Down \\
\hline TC02004814.hg.1 & -1.610212 & 0.000171 & Down \\
\hline TC19000785.hg.1 & -1.687945 & 0.000174 & Down \\
\hline TC12000558.hg.1 & -1.462384 & 0.000176 & Down \\
\hline TC02003744.hg.1 & 1.609164 & 0.000179 & Up \\
\hline TC10000962.hg.1 & -1.497291 & 0.000182 & Down \\
\hline TC12000231.hg.1 & -1.45832 & 0.000184 & Down \\
\hline TC11003109.hg.1 & 2.194265 & 0.000187 & Up \\
\hline TC09001602.hg.1 & -1.532716 & 0.000189 & Down \\
\hline TC20000650.hg.1 & -1.709435 & 0.000192 & Down \\
\hline TCOX001292.hg.1 & -2.223119 & 0.000195 & Down \\
\hline TC17000491.hg.1 & -1.571049 & 0.000197 & Down \\
\hline TC02001836.hg.1 & -1.460727 & $2.00 E-04$ & Down \\
\hline TC02001799.hg.1 & -1.884772 & 0.000202 & Down \\
\hline TC1 1002486.hg.1 & -1.617606 & 0.000205 & Down \\
\hline TC05001562.hg.1 & -1.506318 & 0.000208 & Down \\
\hline TC19000163.hg.1 & -1.58983 & 0.00021 & Down \\
\hline TC03001571.hg.1 & -1.551407 & 0.000213 & Down \\
\hline TC01002801.hg.1 & 1.387205 & 0.000215 & Up \\
\hline TC10000791.hg.1 & -1.567348 & 0.000218 & Down \\
\hline TC05002279.hg.1 & -1.404635 & 0.000221 & Down \\
\hline TC14000620.hg.1 & -1.492016 & 0.000223 & Down \\
\hline TC21000698.hg.1 & -1.432959 & 0.000226 & Down \\
\hline
\end{tabular}


Table 2 (continued)

\begin{tabular}{|c|c|c|c|}
\hline Probe set ID & Fold change & $p$-value & Gene feature \\
\hline TCOX001976.hg.1 & 1.511003 & 0.000229 & Up \\
\hline TC05003313.hg.1 & 1.520386 & 0.000231 & Up \\
\hline TC22000098.hg.1 & -1.597171 & 0.000234 & Down \\
\hline TC08000801.hg.1 & -1.529742 & 0.000236 & Down \\
\hline TC22000156.hg.1 & 1.691877 & 0.000239 & Up \\
\hline TC16002030.hg.1 & -1.399794 & 0.000242 & Down \\
\hline TCOX001158.hg.1 & -1.925543 & 0.000244 & Down \\
\hline TC06000041.hg.1 & -1.3817 & 0.000247 & Down \\
\hline TC10000866.hg.1 & -1.499786 & 0.000249 & Down \\
\hline TC03000195.hg.1 & -1.569494 & 0.000252 & Down \\
\hline TC01003308.hg.1 & -1.760166 & 0.000255 & Down \\
\hline TC01004112.hg.1 & -1.64911 & 0.000257 & Down \\
\hline TCOX001770.hg.1 & -1.818282 & 0.00026 & Down \\
\hline TC04000508.hg.1 & -1.514532 & 0.000262 & Down \\
\hline TC06000705.hg.1 & -1.536016 & 0.000265 & Down \\
\hline TC02003743.hg.1 & 1.526381 & 0.000268 & Up \\
\hline TC05002800.hg.1 & -1.561318 & 0.00027 & Down \\
\hline TC01005268.hg.1 & 1.82533 & 0.000273 & Up \\
\hline TC08000450.hg.1 & -1.626193 & 0.000276 & Down \\
\hline TC10000439.hg.1 & -1.447509 & 0.000278 & Down \\
\hline TC06004042.hg.1 & -1.43228 & 0.000281 & Down \\
\hline TC09002851.hg.1 & -1.367414 & 0.000283 & Down \\
\hline TC06001651.hg.1 & -1.434151 & 0.000286 & Down \\
\hline TC07003059.hg.1 & 1.731542 & 0.000289 & Up \\
\hline TC10000686.hg.1 & -1.370893 & 0.000291 & Down \\
\hline TC08000385.hg.1 & -1.537691 & 0.000294 & Down \\
\hline TC02001432.hg.1 & -1.513117 & 0.000296 & Down \\
\hline TC01002746.hg.1 & -1.610494 & 0.000299 & Down \\
\hline TC03002116.hg.1 & -1.353733 & 0.000302 & Down \\
\hline TC01005428.hg.1 & 1.560865 & 0.000304 & Up \\
\hline TC11003210.hg.1 & 1.443241 & 0.000307 & Up \\
\hline TC21000149.hg.1 & -1.397728 & 0.00031 & Down \\
\hline TC09001534.hg.1 & -1.579598 & 0.000312 & Down \\
\hline TCOX000450.hg.1 & -1.38553 & 0.000315 & Down \\
\hline TCOX001136.hg.1 & -1.386582 & 0.000317 & Down \\
\hline TC06002801.hg.1 & -1.504601 & 0.00032 & Down \\
\hline TC12002688.hg.1 & -1.609811 & 0.000323 & Down \\
\hline TC04002443.hg.1 & -1.520125 & 0.000325 & Down \\
\hline TC16001253.hg.1 & -1.436957 & 0.000328 & Down \\
\hline TC06002402.hg.1 & 1.593718 & 0.00033 & Up \\
\hline TC04001524.hg.1 & -1.497563 & 0.000333 & Down \\
\hline TC22001417.hg.1 & -1.526196 & 0.000336 & Down \\
\hline TC10001922.hg.1 & 1.256632 & 0.000338 & Up \\
\hline TC02003914.hg.1 & -1.445488 & 0.000341 & Down \\
\hline TC16000510.hg.1 & -1.576476 & 0.000343 & Down \\
\hline TC01002976.hg.1 & -1.493934 & 0.000346 & Down \\
\hline TCOM000020.hg.1 & -5.95641 & 0.000349 & Down \\
\hline TC01002798.hg.1 & -1.77144 & 0.000351 & Down \\
\hline TC01006074.hg.1 & -1.417269 & 0.000354 & Down \\
\hline
\end{tabular}

Table 2 (continued)

\begin{tabular}{|c|c|c|c|}
\hline Probe set ID & Fold change & p-value & Gene feature \\
\hline TC06000568.hg.1 & -1.558205 & 0.000357 & Down \\
\hline TC12000088.hg.1 & 1.369654 & 0.000359 & Up \\
\hline TC16001359.hg.1 & 1.37628 & 0.000362 & Up \\
\hline TC03001321.hg.1 & -1.300817 & 0.000364 & Down \\
\hline TC07002531.hg.1 & 1.474871 & 0.000367 & Up \\
\hline TCOX000233.hg.1 & -1.387042 & 0.00037 & Down \\
\hline TCOX000985.hg.1 & -1.911084 & 0.000372 & Down \\
\hline TCOX001762.hg.1 & -1.495234 & 0.000375 & Down \\
\hline TC05000067.hg.1 & 1.605557 & 0.000377 & Up \\
\hline TC09001461.hg.1 & -1.504051 & 0.00038 & Down \\
\hline TC15000651.hg.1 & -1.579594 & 0.000383 & Down \\
\hline TCOX001889.hg.1 & 1.389217 & 0.000385 & Up \\
\hline TC15002609.hg.1 & 1.387993 & 0.000388 & Up \\
\hline TC14000925.hg.1 & -1.487896 & 0.00039 & Down \\
\hline TC22001175.hg.1 & -1.488885 & 0.000393 & Down \\
\hline TC04001945.hg.1 & -1.669152 & 0.000396 & Down \\
\hline TC09000096.hg.1 & -1.861195 & 0.000398 & Down \\
\hline TC05002818.hg.1 & -1.472401 & 0.000401 & Down \\
\hline TC07002463.hg.1 & 1.484579 & 0.000404 & Up \\
\hline TC01003990.hg.1 & -1.543233 & 0.000406 & Down \\
\hline TC06003126.hg.1 & -1.312105 & 0.000409 & Down \\
\hline TC12000805.hg.1 & -1.554705 & 0.000411 & Down \\
\hline TC17002196.hg.1 & 1.345657 & 0.000414 & Up \\
\hline TC04000811.hg.1 & -1.321197 & 0.000422 & Down \\
\hline TC22000646.hg.1 & 1.223546 & 0.000424 & Up \\
\hline TC1 1003382.hg.1 & 1.556575 & 0.000427 & Up \\
\hline TC15001184.hg.1 & -1.306114 & 0.00043 & Down \\
\hline TC01000388.hg.1 & 1.394364 & 0.000432 & Up \\
\hline TC03003257.hg.1 & -1.314749 & 0.000435 & Down \\
\hline TC08000285.hg.1 & -1.561996 & 0.000437 & Down \\
\hline TCOX001410.hg.1 & -1.54477 & 0.00044 & Down \\
\hline TC07000478.hg.1 & -1.522336 & 0.000443 & Down \\
\hline TC12002858.hg.1 & 1.359725 & 0.000448 & Up \\
\hline TC03001056.hg.1 & -1.423964 & 0.000451 & Down \\
\hline TC15001493.hg.1 & -1.264085 & 0.000456 & Down \\
\hline TC05001001.hg.1 & -1.366729 & 0.000458 & Down \\
\hline TC02001858.hg.1 & -1.336156 & 0.000461 & Down \\
\hline TC10002369.hg.1 & 1.416824 & 0.000464 & Up \\
\hline TC01002620.hg.1 & -1.372948 & 0.000466 & Down \\
\hline TC12001449.hg.1 & -1.559039 & 0.000477 & Down \\
\hline TC15002177.hg.1 & 1.204774 & 0.000479 & Up \\
\hline TC10001021.hg.1 & -1.351391 & 0.000482 & Down \\
\hline TCOX002006.hg.1 & -1.276702 & 0.000487 & Down \\
\hline TCOM000021.hg.1 & -1.76836 & 0.000492 & Down \\
\hline TC02001302.hg.1 & -1.500158 & 0.000498 & Down \\
\hline TC07000690.hg.1 & -1.464123 & 0.000505 & Down \\
\hline TC05000861.hg.1 & -1.43654 & 0.000508 & Down \\
\hline TC05003112.hg.1 & -1.883715 & 0.000511 & Down \\
\hline TC22000400.hg.1 & -1.397564 & 0.000513 & Down \\
\hline
\end{tabular}


Table 2 (continued)

\begin{tabular}{|c|c|c|c|}
\hline Probe set ID & Fold change & $p$-value & Gene feature \\
\hline TC14001892.hg.1 & -1.409308 & 0.000516 & Down \\
\hline TC11002784.hg.1 & 1.463689 & 0.000518 & Up \\
\hline TCOX001907.hg.1 & 1.631743 & 0.000526 & Up \\
\hline TCOX001411.hg.1 & -1.575078 & 0.000529 & Down \\
\hline TC06000972.hg.1 & -1.412607 & 0.000532 & Down \\
\hline TC07000702.hg.1 & -1.574012 & 0.000534 & Down \\
\hline TC15001073.hg.1 & -1.487709 & 0.000537 & Down \\
\hline TC08002416.hg.1 & -1.30562 & 0.000539 & Down \\
\hline TC05000611.hg.1 & -1.342755 & 0.000542 & Down \\
\hline TC09001256.hg.1 & -1.245674 & 0.000545 & Down \\
\hline TCOM000023.hg.1 & -1.665398 & 0.000552 & Down \\
\hline TC16001522.hg.1 & -1.550585 & 0.000555 & Down \\
\hline TC02001429.hg.1 & -1.384528 & 0.000558 & Down \\
\hline TC05000688.hg.1 & -1.465004 & 0.00056 & Down \\
\hline TC07001655.hg.1 & -1.483501 & 0.000563 & Down \\
\hline TC09002148.hg.1 & -1.294238 & 0.000565 & Down \\
\hline TC03002425.hg.1 & -1.414502 & 0.000568 & Down \\
\hline TC17002307.hg.1 & 1.574493 & 0.000571 & Up \\
\hline TC11002476.hg.1 & -1.407878 & 0.000573 & Down \\
\hline TC07002321.hg.1 & 1.340462 & 0.000576 & Up \\
\hline TC10001073.hg.1 & 1.420479 & 0.000579 & Up \\
\hline TC13001025.hg.1 & 1.281803 & 0.000581 & Up \\
\hline TC18000050.hg.1 & -1.43278 & 0.000586 & Down \\
\hline TC08001833.hg.1 & 1.301019 & 0.000589 & Up \\
\hline TC02001366.hg.1 & -1.411719 & 0.000594 & Down \\
\hline TC09002445.hg.1 & -1.365813 & 0.000597 & Down \\
\hline TC12000244.hg.1 & -1.456782 & 0.000599 & Down \\
\hline TC16000919.hg.1 & -1.412488 & 0.000602 & Down \\
\hline TC01001291.hg.1 & 1.30011 & 0.000605 & Up \\
\hline TC04002772.hg.1 & -1.318518 & 0.000607 & Down \\
\hline TC09001898.hg.1 & -1.355328 & 0.00061 & Down \\
\hline TC02000784.hg.1 & 1.244517 & 0.000612 & Up \\
\hline TCOX001210.hg.1 & -1.297482 & 0.000615 & Down \\
\hline TC03001672.hg.1 & -1.300852 & 0.00062 & Down \\
\hline TC16001991.hg.1 & -1.312834 & 0.000623 & Down \\
\hline TC20001376.hg.1 & 1.259521 & 0.000626 & Up \\
\hline TC12001995.hg.1 & -1.534637 & 0.000631 & Down \\
\hline TC20001199.hg.1 & -1.438895 & 0.000633 & Down \\
\hline TC07001788.hg.1 & -1.605406 & 0.000639 & Down \\
\hline TC08001734.hg.1 & -1.313819 & 0.000641 & Down \\
\hline TC07001706.hg.1 & -1.621814 & 0.000645 & Down \\
\hline TC07001709.hg.1 & -1.621814 & 0.000645 & Down \\
\hline TC03002939.hg.1 & 1.465012 & 0.000649 & Up \\
\hline TC11000808.hg.1 & -1.255062 & 0.000652 & Down \\
\hline TC02002028.hg.1 & -1.408438 & 0.000654 & Down \\
\hline TC02002581.hg.1 & -1.345698 & 0.000657 & Down \\
\hline TC01005437.hg.1 & -1.25784 & 0.000659 & Down \\
\hline TC18000864.hg.1 & 1.420831 & 0.000662 & Up \\
\hline TC03003057.hg.1 & 1.297577 & 0.000667 & Up \\
\hline
\end{tabular}

Table 2 (continued)

\begin{tabular}{|c|c|c|c|}
\hline Probe set ID & Fold change & p-value & Gene feature \\
\hline TC1 1002077.hg.1 & -1.516075 & 0.00067 & Down \\
\hline TCOX001618.hg.1 & -1.55017 & 0.000673 & Down \\
\hline TC16002013.hg.1 & -1.464825 & 0.000675 & Down \\
\hline TC02002957.hg.1 & -1.21553 & 0.000678 & Down \\
\hline TC14001601.hg.1 & -1.266846 & 0.00068 & Down \\
\hline TC06003365.hg.1 & 1.258709 & 0.000683 & Up \\
\hline TC02001623.hg.1 & -1.387294 & 0.000691 & Down \\
\hline TCOX001430.hg.1 & -1.517692 & 0.000693 & Down \\
\hline TC02001248.hg.1 & -1.343015 & 0.000701 & Down \\
\hline TC07000139.hg.1 & -1.548066 & 0.000704 & Down \\
\hline TC08001239.hg.1 & -1.411515 & 0.000709 & Down \\
\hline TC16001996.hg.1 & -1.32531 & 0.000714 & Down \\
\hline TC07001848.hg.1 & -1.223116 & 0.000717 & Down \\
\hline TC17001317.hg.1 & -1.258521 & 0.00072 & Down \\
\hline TC06001365.hg.1 & -1.568292 & 0.000722 & Down \\
\hline TC09000783.hg.1 & -1.241694 & 0.000725 & Down \\
\hline TC03003056.hg.1 & 1.309714 & 0.000727 & Up \\
\hline TC1 1001141.hg.1 & -1.474463 & 0.00073 & Down \\
\hline TC15000819.hg.1 & -1.466781 & 0.000733 & Down \\
\hline TC17000164.hg.1 & -1.381715 & 0.000743 & Down \\
\hline TC10002136.hg.1 & -1.222626 & 0.000748 & Down \\
\hline TC12000238.hg.1 & -1.398297 & 0.000751 & Down \\
\hline TC19002149.hg.1 & 1.274434 & 0.000754 & Up \\
\hline TC10001432.hg.1 & -1.395271 & 0.000756 & Down \\
\hline TC12002396.hg.1 & 1.37332 & 0.000759 & Up \\
\hline TC06002303.hg.1 & -1.481066 & 0.000767 & Down \\
\hline TC02002233.hg.1 & 1.281339 & 0.000769 & Up \\
\hline TC05002034.hg.1 & -1.450196 & 0.000772 & Down \\
\hline TC03002972.hg.1 & -1.372256 & 0.000777 & Down \\
\hline TC02001721.hg.1 & -1.367369 & 0.00078 & Down \\
\hline TC05001948.hg.1 & -1.229624 & 0.000782 & Down \\
\hline TC06003416.hg.1 & -1.25784 & 0.000785 & Down \\
\hline TC05000214.hg.1 & -1.345182 & 0.000787 & Down \\
\hline TCOX002258.hg.1 & -1.40134 & 0.000795 & Down \\
\hline TC13001479.hg.1 & 1.612735 & 0.000798 & Up \\
\hline TC01004166.hg.1 & -1.350561 & 0.000803 & Down \\
\hline TC15000333.hg.1 & 1.346913 & 0.000806 & Up \\
\hline TC18000381.hg.1 & -1.459511 & 0.000808 & Down \\
\hline TCOX001739.hg.1 & -1.221974 & 0.000821 & Down \\
\hline TC04000854.hg.1 & -1.342571 & 0.000824 & Down \\
\hline TC14001245.hg.1 & -1.363644 & 0.000827 & Down \\
\hline TC16000853.hg.1 & -1.526047 & 0.000829 & Down \\
\hline TC02004957.hg.1 & -1.256608 & 0.000832 & Down \\
\hline TC16000648.hg.1 & -1.967375 & 0.000834 & Down \\
\hline TC01002757.hg.1 & -1.315911 & 0.000837 & Down \\
\hline TC05000183.hg.1 & -1.466612 & 0.000842 & Down \\
\hline TC08000652.hg.1 & -1.444517 & 0.000845 & Down \\
\hline TC12003194.hg.1 & -1.396169 & 0.00085 & Down \\
\hline TC01002633.hg.1 & -1.459449 & 0.000853 & Down \\
\hline
\end{tabular}


Table 2 (continued)

\begin{tabular}{|c|c|c|c|}
\hline Probe set ID & Fold change & p-value & Gene feature \\
\hline TC02004273.hg.1 & -1.239977 & 0.000855 & Down \\
\hline TCOX000473.hg.1 & -1.208647 & 0.000863 & Down \\
\hline TC01001013.hg.1 & 1.402441 & 0.000866 & Up \\
\hline TC07003162.hg.1 & -1.286344 & 0.000871 & Down \\
\hline TC20000340.hg.1 & -1.467848 & 0.000874 & Down \\
\hline TC02000955.hg.1 & -1.516537 & 0.000876 & Down \\
\hline TC17001429.hg.1 & -1.288095 & 0.000884 & Down \\
\hline TC02002970.hg.1 & -1.262033 & 0.000887 & Down \\
\hline TC19000423.hg.1 & -1.270008 & 0.000892 & Down \\
\hline TC04002642.hg.1 & -1.379802 & 0.000895 & Down \\
\hline TC14000739.hg.1 & 1.321768 & 0.000902 & Up \\
\hline TC09001812.hg.1 & -1.231765 & 0.000905 & Down \\
\hline TC15001577.hg.1 & -1.461504 & 0.000908 & Down \\
\hline TC12003053.hg.1 & 1.319866 & 0.00091 & Up \\
\hline TC12002444.hg.1 & 1.329822 & 0.000913 & Up \\
\hline TC07001462.hg.1 & 1.464142 & 0.000915 & Up \\
\hline TC12001455.hg.1 & -1.443029 & 0.000918 & Down \\
\hline TC04000571.hg.1 & -1.489108 & 0.000921 & Down \\
\hline TCOX001193.hg.1 & 1.282141 & 0.000923 & Up \\
\hline TC17002577.hg.1 & -1.222474 & 0.000929 & Down \\
\hline TCOX000986.hg.1 & -1.537172 & 0.000934 & Down \\
\hline TC18000372.hg.1 & 1.306631 & 0.000936 & Up \\
\hline TC12003159.hg.1 & -1.26419 & 0.000944 & Down \\
\hline TC12001777.hg.1 & -1.387482 & 0.000947 & Down \\
\hline TC17000629.hg.1 & 1.506256 & 0.000952 & Up \\
\hline TC01002397.hg.1 & -1.298179 & 0.000955 & Down \\
\hline TC11000089.hg.1 & -1.293079 & 0.00096 & Down \\
\hline TC19001173.hg.1 & -1.788733 & 0.000962 & Down \\
\hline TC07002196.hg.1 & -1.237867 & 0.000965 & Down \\
\hline TC01004898.hg.1 & 1.21174 & 0.000968 & Up \\
\hline TC15002247.hg.1 & 1.224493 & 0.000976 & Up \\
\hline TC05001142.hg.1 & -1.326114 & 0.000978 & Down \\
\hline TC05000621.hg.1 & -1.464508 & 0.000989 & Down \\
\hline TC09000642.hg.1 & -1.540151 & 0.000991 & Down \\
\hline TC09001936.hg.1 & -1.364479 & 0.000994 & Down \\
\hline TC17002761.hg.1 & -1.269107 & 0.000999 & Down \\
\hline TC04002187.hg.1 & 1.408721 & 0.001002 & Up \\
\hline TC02003570.hg.1 & -1.280663 & 0.001004 & Down \\
\hline TCOX001616.hg.1 & -1.322208 & 0.001007 & Down \\
\hline TC09001972.hg.1 & -1.209848 & 0.001012 & Down \\
\hline TC06003710.hg.1 & -1.497029 & 0.001015 & Down \\
\hline TC03001944.hg.1 & 1.214647 & 0.001017 & Up \\
\hline TC01004109.hg.1 & -1.321069 & 0.00102 & Down \\
\hline TC17002195.hg.1 & 1.21302 & 0.001023 & Up \\
\hline TC05002180.hg.1 & -1.221883 & 0.001036 & Down \\
\hline TC20001446.hg.1 & 1.296941 & 0.001038 & Up \\
\hline TC09002470.hg.1 & 1.352053 & 0.001041 & Up \\
\hline TC08001621.hg.1 & -1.246738 & 0.001043 & Down \\
\hline TC02004746.hg.1 & 1.298033 & 0.001046 & Up \\
\hline
\end{tabular}

Table 2 (continued)

\begin{tabular}{|c|c|c|c|}
\hline Probe set ID & Fold change & p-value & Gene feature \\
\hline TC10000082.hg.1 & -1.243931 & 0.001051 & Down \\
\hline TC08001847.hg.1 & 1.362437 & 0.001056 & Up \\
\hline TC10002381.hg.1 & -1.21549 & 0.001062 & Down \\
\hline TC14001177.hg.1 & -1.270448 & 0.001064 & Down \\
\hline TC10000872.hg.1 & -1.230721 & 0.00107 & Down \\
\hline TC06000025.hg.1 & 1.328452 & 0.001075 & Up \\
\hline TC01005249.hg.1 & -1.213079 & 0.001083 & Down \\
\hline TC16001555.hg.1 & -1.208988 & 0.00109 & Down \\
\hline TCOY000124.hg.1 & 1.246549 & 0.001093 & Up \\
\hline TC1 1001732.hg.1 & -1.318377 & 0.001096 & Down \\
\hline TC03000629.hg.1 & -1.439342 & 0.001098 & Down \\
\hline TC09001843.hg.1 & -1.204212 & 0.001104 & Down \\
\hline TC09002783.hg.1 & -1.269006 & 0.001106 & Down \\
\hline TC01004933.hg.1 & 1.342382 & 0.001109 & Up \\
\hline TC10000236.hg.1 & -1.319501 & 0.001114 & Down \\
\hline TC05001371.hg.1 & -1.314131 & 0.001122 & Down \\
\hline TC14000052.hg.1 & -1.409573 & 0.001124 & Down \\
\hline TC21000931.hg.1 & -1.264853 & 0.00113 & Down \\
\hline TC03002997.hg.1 & 1.270405 & 0.001132 & Up \\
\hline TC05003198.hg.1 & -1.253982 & 0.001135 & Down \\
\hline TC02002711.hg.1 & -1.272432 & 0.001137 & Down \\
\hline TC02004784.hg.1 & -1.200546 & 0.001143 & Down \\
\hline TC05002575.hg.1 & 1.219313 & 0.001145 & Up \\
\hline TC01002594.hg.1 & -1.421401 & 0.001148 & Down \\
\hline TC16001826.hg.1 & -1.353943 & 0.001169 & Down \\
\hline TC08001640.hg.1 & -1.376088 & 0.001171 & Down \\
\hline TC17002837.hg.1 & -1.262026 & 0.001174 & Down \\
\hline TC08001247.hg.1 & -1.233191 & 0.001177 & Down \\
\hline TC07000624.hg.1 & -1.263829 & 0.001179 & Down \\
\hline TC12001263.hg.1 & -1.216333 & 0.001184 & Down \\
\hline TC06000824.hg.1 & -1.41643 & 0.00119 & Down \\
\hline TC12000184.hg.1 & -1.273853 & 0.001192 & Down \\
\hline TC12003089.hg.1 & -1.237444 & 0.001208 & Down \\
\hline TC10000369.hg.1 & -1.232303 & 0.001218 & Down \\
\hline TC03000823.hg.1 & -1.318296 & 0.001231 & Down \\
\hline TCOX000808.hg.1 & -1.294835 & 0.001237 & Down \\
\hline TC15001473.hg.1 & -1.390182 & 0.001239 & Down \\
\hline TC01006043.hg.1 & -1.279341 & 0.001242 & Down \\
\hline TC16001156.hg.1 & -1.347061 & 0.001245 & Down \\
\hline TC05000708.hg.1 & -1.457383 & 0.001247 & Down \\
\hline TC07002757.hg.1 & -1.342035 & 0.00125 & Down \\
\hline TC01003748.hg.1 & -1.247279 & 0.001252 & Down \\
\hline TC20000045.hg.1 & -1.611347 & 0.001255 & Down \\
\hline TC19000349.hg.1 & -1.294863 & 0.001258 & Down \\
\hline TC08002335.hg.1 & -1.575981 & 0.001273 & Down \\
\hline TC02003970.hg.1 & -1.261765 & 0.001276 & Down \\
\hline TC07002353.hg.1 & -1.21386 & 0.001292 & Down \\
\hline TC03000155.hg.1 & -1.308938 & 0.001294 & Down \\
\hline TCOX002344.hg.1 & -1.336301 & 0.001299 & Down \\
\hline
\end{tabular}


Table 2 (continued)

\begin{tabular}{|c|c|c|c|}
\hline Probe set ID & Fold change & $p$-value & Gene feature \\
\hline TC01004110.hg.1 & -1.549747 & 0.00131 & Down \\
\hline TC12000390.hg.1 & -1.251394 & 0.001318 & Down \\
\hline TC14000427.hg.1 & -1.249078 & 0.001323 & Down \\
\hline TC03000342.hg.1 & -1.293203 & 0.001326 & Down \\
\hline TC09001279.hg.1 & -1.291408 & 0.001331 & Down \\
\hline TC14000693.hg.1 & -1.497742 & 0.001344 & Down \\
\hline TC12003094.hg.1 & -1.417707 & 0.001349 & Down \\
\hline TC06002606.hg.1 & -1.537348 & 0.001352 & Down \\
\hline TC06001540.hg.1 & -1.242118 & 0.001354 & Down \\
\hline TC03001808.hg.1 & -1.327132 & 0.001359 & Down \\
\hline TC10000793.hg.1 & -1.384112 & 0.001362 & Down \\
\hline TC20001 166.hg.1 & -1.216393 & 0.001383 & Down \\
\hline TC01005264.hg.1 & -1.316901 & 0.001388 & Down \\
\hline TC09000471.hg.1 & -1.31262 & 0.001404 & Down \\
\hline TC19002342.hg.1 & -1.360527 & 0.00142 & Down \\
\hline TCOX001450.hg.1 & -1.226402 & 0.001433 & Down \\
\hline TCOX001094.hg.1 & -1.281421 & 0.001448 & Down \\
\hline TC0X000282.hg.1 & -1.397268 & 0.001451 & Down \\
\hline TC16001699.hg.1 & -1.21053 & 0.001459 & Down \\
\hline TC08000411.hg.1 & -1.264325 & 0.001477 & Down \\
\hline TC01003906.hg.1 & -1.277746 & 0.001485 & Down \\
\hline TC02001952.hg.1 & -1.202124 & 0.001487 & Down \\
\hline TC03002704.hg.1 & -1.2066 & 0.00149 & Down \\
\hline TC06002227.hg.1 & -1.536921 & 0.001493 & Down \\
\hline TC16000560.hg.1 & -1.319732 & 0.001501 & Down \\
\hline TCOX001939.hg.1 & -1.239263 & 0.001503 & Down \\
\hline TC1 1002900.hg.1 & -1.422263 & 0.001506 & Down \\
\hline TC09000516.hg.1 & -1.3743 & 0.001521 & Down \\
\hline TC20000564.hg.1 & -1.259432 & 0.001529 & Down \\
\hline TC08000425.hg.1 & -1.222783 & 0.001532 & Down \\
\hline TC07000862.hg.1 & -1.318867 & 0.001534 & Down \\
\hline TC12001821.hg.1 & -1.267134 & 0.001545 & Down \\
\hline TC11000653.hg.1 & -1.256931 & 0.001548 & Down \\
\hline TC02004514.hg.1 & -1.278303 & 0.00155 & Down \\
\hline TC05002588.hg.1 & -1.361963 & 0.001553 & Down \\
\hline TC06002096.hg.1 & -1.234314 & 0.001561 & Down \\
\hline TC10001912.hg.1 & -1.30431 & 0.001563 & Down \\
\hline TC02000188.hg.1 & -1.343934 & 0.001566 & Down \\
\hline TC03000381.hg.1 & -1.236027 & 0.0016 & Down \\
\hline TC20000763.hg.1 & -1.225856 & 0.001602 & Down \\
\hline TC19000786.hg.1 & -1.255489 & 0.001608 & Down \\
\hline TC08000224.hg.1 & -1.273889 & 0.00161 & Down \\
\hline TC12001052.hg.1 & -1.452174 & 0.001613 & Down \\
\hline TC07000779.hg.1 & -1.324442 & 0.001644 & Down \\
\hline TC12002368.hg.1 & -1.228785 & 0.001647 & Down \\
\hline TC02001190.hg.1 & -1.338948 & 0.001652 & Down \\
\hline TC05000526.hg.1 & -1.221816 & 0.001665 & Down \\
\hline TC04002294.hg.1 & -1.301327 & 0.001668 & Down \\
\hline TC21000706.hg.1 & -1.298316 & 0.001686 & Down \\
\hline
\end{tabular}

Table 2 (continued)

\begin{tabular}{|c|c|c|c|}
\hline Probe set ID & Fold change & p-value & Gene feature \\
\hline TC02002768.hg.1 & -1.312801 & 0.001694 & Down \\
\hline TC17001656.hg.1 & -1.267211 & 0.001702 & Down \\
\hline TC03001494.hg.1 & -1.366098 & 0.00172 & Down \\
\hline TC13000481.hg.1 & -1.28573 & 0.001723 & Down \\
\hline TC16000776.hg.1 & -1.273221 & 0.001725 & Down \\
\hline TC10002723.hg.1 & -1.376529 & 0.001728 & Down \\
\hline TC10002728.hg.1 & -1.244225 & 0.001733 & Down \\
\hline TC01006051.hg.1 & -1.250802 & 0.001736 & Down \\
\hline TC05001849.hg.1 & -1.217863 & 0.001743 & Down \\
\hline TC10000232.hg.1 & -1.310615 & 0.001751 & Down \\
\hline TC01003920.hg.1 & -1.223489 & 0.001779 & Down \\
\hline TC01003921.hg.1 & -1.223489 & 0.001779 & Down \\
\hline TC01003922.hg.1 & -1.223489 & 0.001779 & Down \\
\hline TC01003923.hg.1 & -1.223489 & 0.001779 & Down \\
\hline TC01003924.hg.1 & -1.223489 & 0.001779 & Down \\
\hline TC01003925.hg.1 & -1.223489 & 0.001779 & Down \\
\hline TC01003926.hg.1 & -1.223489 & 0.001779 & Down \\
\hline TC01003927.hg.1 & -1.223489 & 0.001779 & Down \\
\hline TC01003929.hg.1 & -1.223489 & 0.001779 & Down \\
\hline TC01003930.hg.1 & -1.223489 & 0.001779 & Down \\
\hline TC01003931.hg.1 & -1.223489 & 0.001779 & Down \\
\hline TC01003932.hg.1 & -1.223489 & 0.001779 & Down \\
\hline TC01003933.hg.1 & -1.223489 & 0.001779 & Down \\
\hline TC01003934.hg.1 & -1.223489 & 0.001779 & Down \\
\hline TC01003935.hg.1 & -1.223489 & 0.001779 & Down \\
\hline TC01003936.hg.1 & -1.223489 & 0.001779 & Down \\
\hline TC03003030.hg.1 & -1.478844 & 0.001803 & Down \\
\hline TC14000845.hg.1 & -1.312238 & 0.001806 & Down \\
\hline TC05002975.hg.1 & -1.285007 & 0.001809 & Down \\
\hline TC20000261.hg.1 & -1.329768 & 0.001817 & Down \\
\hline TC14001281.hg.1 & -1.272863 & 0.001819 & Down \\
\hline TC05000998.hg.1 & -1.275857 & 0.00183 & Down \\
\hline TC13001120.hg.1 & -1.345262 & 0.001832 & Down \\
\hline TC03000057.hg.1 & -1.226194 & 0.001853 & Down \\
\hline TC1 1000768.hg.1 & -1.347505 & 0.001856 & Down \\
\hline TC04001473.hg.1 & -1.385031 & 0.001861 & Down \\
\hline TC08001306.hg.1 & -1.237854 & 0.001864 & Down \\
\hline TC02004490.hg.1 & -1.228885 & 0.001866 & Down \\
\hline TC02004794.hg.1 & -1.244544 & 0.001877 & Down \\
\hline TC08001540.hg.1 & -1.312764 & 0.001884 & Down \\
\hline TC03000875.hg.1 & -1.363667 & 0.001898 & Down \\
\hline TC06001064.hg.1 & -1.411292 & 0.001903 & Down \\
\hline TC08000407.hg.1 & -1.291451 & 0.001905 & Down \\
\hline TC10002820.hg.1 & -1.233983 & 0.001908 & Down \\
\hline TC14000661.hg.1 & -1.226019 & 0.001937 & Down \\
\hline TC19000244.hg.1 & -1.222774 & 0.001939 & Down \\
\hline TC09000897.hg.1 & -1.241196 & 0.001952 & Down \\
\hline TC1 1002142.hg.1 & -1.530933 & 0.001978 & Down \\
\hline TC04000338.hg.1 & -1.28848 & 0.001984 & Down \\
\hline
\end{tabular}


Table 2 (continued)

\begin{tabular}{|c|c|c|c|}
\hline Probe set ID & Fold change & p-value & Gene feature \\
\hline TC12002736.hg.1 & -1.306143 & 0.001989 & Down \\
\hline TC09000458.hg.1 & -1.260067 & 0.002005 & Down \\
\hline TC19002478.hg.1 & -1.251909 & 0.002012 & Down \\
\hline TC17000607.hg.1 & -1.376805 & 0.00202 & Down \\
\hline TC08002174.hg.1 & -1.252071 & 0.002049 & Down \\
\hline TC15002522.hg.1 & -1.352134 & 0.002059 & Down \\
\hline TC07001721.hg.1 & -1.27015 & 0.002099 & Down \\
\hline TC11000172.hg.1 & -1.297266 & 0.002101 & Down \\
\hline TCOM000022.hg.1 & -1.790883 & 0.002127 & Down \\
\hline TC01000642.hg.1 & -1.273087 & 0.002135 & Down \\
\hline TC04002779.hg.1 & -1.295919 & 0.002172 & Down \\
\hline TC17000230.hg.1 & -1.28801 & 0.002177 & Down \\
\hline TC10002673.hg.1 & -1.341757 & 0.002185 & Down \\
\hline TC02000761.hg.1 & -1.200639 & 0.00219 & Down \\
\hline TC07001870.hg.1 & -1.503979 & 0.002198 & Down \\
\hline TC13001485.hg.1 & -1.322513 & 0.0022 & Down \\
\hline TCOX002104.hg.1 & -1.235461 & 0.002203 & Down \\
\hline TC01003794.hg.1 & -1.275671 & 0.002216 & Down \\
\hline TC14000809.hg.1 & -1.232224 & 0.002247 & Down \\
\hline TC08001563.hg.1 & -1.281014 & 0.002292 & Down \\
\hline TC02003041.hg.1 & -1.439696 & 0.002305 & Down \\
\hline TC01000878.hg.1 & -1.200347 & 0.002313 & Down \\
\hline TC16001204.hg.1 & -1.216715 & 0.002315 & Down \\
\hline TC10001355.hg.1 & -1.266825 & 0.002318 & Down \\
\hline TC10001940.hg.1 & -1.302501 & 0.002334 & Down \\
\hline TC04000456.hg.1 & -1.259762 & 0.002347 & Down \\
\hline TC19001288.hg.1 & -1.211289 & 0.002355 & Down \\
\hline TC21000403.hg.1 & -1.262158 & 0.002365 & Down \\
\hline TC15001614.hg.1 & -1.242348 & 0.00237 & Down \\
\hline TC01002339.hg.1 & -1.287548 & 0.002373 & Down \\
\hline TC21000126.hg.1 & -1.220964 & 0.002375 & Down \\
\hline TC04001229.hg.1 & -1.226388 & 0.002399 & Down \\
\hline TC10002579.hg.1 & -1.204095 & 0.002422 & Down \\
\hline TC01003671.hg.1 & -1.209995 & 0.00243 & Down \\
\hline TC01001982.hg.1 & -1.217144 & 0.002436 & Down \\
\hline TC15001480.hg.1 & -1.259755 & 0.002438 & Down \\
\hline TC22001201.hg.1 & -1.534217 & 0.002443 & Down \\
\hline TC10000404.hg.1 & -1.319697 & 0.002472 & Down \\
\hline TC01000565.hg.1 & -1.208333 & 0.002493 & Down \\
\hline TC11001288.hg.1 & -1.381912 & 0.002496 & Down \\
\hline TC08001096.hg.1 & -1.310737 & 0.002532 & Down \\
\hline TC17001148.hg.1 & -1.273032 & 0.002535 & Down \\
\hline TC22001301.hg.1 & -1.306173 & 0.002566 & Down \\
\hline TC04002309.hg.1 & -1.209813 & 0.002595 & Down \\
\hline TC21000144.hg.1 & -1.230499 & 0.0026 & Down \\
\hline TC20000800.hg.1 & -1.228074 & 0.002613 & Down \\
\hline TC10001508.hg.1 & -1.242578 & 0.002629 & Down \\
\hline TC11001429.hg.1 & -1.21474 & 0.002699 & Down \\
\hline TC10001326.hg.1 & -1.260137 & 0.002712 & Down \\
\hline
\end{tabular}

Table 2 (continued)

\begin{tabular}{|c|c|c|c|}
\hline Probe set ID & Fold change & p-value & Gene feature \\
\hline TC16001788.hg.1 & -1.266336 & 0.00272 & Down \\
\hline TC01000718.hg.1 & -1.246934 & 0.002731 & Down \\
\hline TC02004391.hg.1 & -1.248587 & 0.002752 & Down \\
\hline TC02004870.hg.1 & -1.393997 & 0.002775 & Down \\
\hline TC02004787.hg.1 & -1.417426 & 0.002791 & Down \\
\hline TC12000361.hg.1 & -1.251152 & 0.002793 & Down \\
\hline TC15000227.hg.1 & -1.256604 & 0.002806 & Down \\
\hline TC01001745.hg.1 & -1.285385 & 0.002814 & Down \\
\hline TC05002924.hg.1 & -1.252245 & 0.002817 & Down \\
\hline TC07002052.hg.1 & -1.237299 & 0.00283 & Down \\
\hline TC03002295.hg.1 & -1.273649 & 0.002848 & Down \\
\hline TC06001529.hg.1 & -1.485667 & 0.002863 & Down \\
\hline $\begin{array}{l}\text { TC6_apd_ } \\
\text { hap1000080.hg.1 }\end{array}$ & -1.485667 & 0.002863 & Down \\
\hline $\begin{array}{l}\text { TC6_cox_ } \\
\text { hap2000158.hg.1 }\end{array}$ & -1.485667 & 0.002863 & Down \\
\hline $\begin{array}{l}\text { TC6_dbb_ } \\
\text { hap3000147.hg.1 }\end{array}$ & -1.485667 & 0.002863 & Down \\
\hline $\begin{array}{l}\text { TC6_mann_- } \\
\text { hap4000135.hg.1 }\end{array}$ & -1.485667 & 0.002863 & Down \\
\hline $\begin{array}{l}\text { TC6_mcf_- } \\
\text { hap5000135.hg.1 }\end{array}$ & -1.485667 & 0.002863 & Down \\
\hline $\begin{array}{l}\text { TC6_qbl_} \\
\text { hap6000150.hg.1 }\end{array}$ & -1.485667 & 0.002863 & Down \\
\hline $\begin{array}{l}\text { TC6_ssto_ } \\
\text { hap7000130.hg.1 }\end{array}$ & -1.485667 & 0.002863 & Down \\
\hline TC07002428.hg.1 & -1.377767 & 0.002882 & Down \\
\hline TC08000954.hg.1 & -1.303647 & 0.002911 & Down \\
\hline TC06002505.hg.1 & -1.254488 & 0.002927 & Down \\
\hline TC21000447.hg.1 & -1.250815 & 0.002953 & Down \\
\hline TC17001238.hg.1 & -1.230333 & 0.002955 & Down \\
\hline TC15002586.hg.1 & -1.305785 & 0.002963 & Down \\
\hline TC12000249.hg.1 & -1.225331 & 0.002976 & Down \\
\hline TC11002394.hg.1 & -1.214327 & 0.002989 & Down \\
\hline TC01005988.hg.1 & -1.33252 & 0.003005 & Down \\
\hline TC21000081.hg.1 & -1.227667 & 0.003015 & Down \\
\hline TC08000521.hg.1 & -1.204106 & 0.003023 & Down \\
\hline TC09000907.hg.1 & -1.244625 & 0.003026 & Down \\
\hline TC14000688.hg.1 & -1.223768 & 0.003062 & Down \\
\hline TC03000666.hg.1 & -1.298135 & 0.003065 & Down \\
\hline TC09000250.hg.1 & -1.206244 & 0.003078 & Down \\
\hline TC01004185.hg.1 & -1.401887 & 0.003086 & Down \\
\hline TC15002417.hg.1 & -1.211724 & 0.003089 & Down \\
\hline TC06003509.hg.1 & -1.227037 & 0.003094 & Down \\
\hline TC01004296.hg.1 & -1.218277 & 0.003143 & Down \\
\hline TC16000068.hg.1 & -1.254429 & 0.003169 & Down \\
\hline TC01002874.hg.1 & -1.282112 & 0.003198 & Down \\
\hline TC03000249.hg.1 & -1.287577 & 0.003209 & Down \\
\hline TC16001224.hg.1 & -1.33179 & 0.003219 & Down \\
\hline TC21000483.hg.1 & -1.416389 & 0.003224 & Down \\
\hline TC14001345.hg.1 & -1.283492 & 0.003237 & Down \\
\hline
\end{tabular}


Table 2 (continued)

\begin{tabular}{|c|c|c|c|}
\hline Probe set ID & Fold change & p-value & Gene feature \\
\hline TC16001268.hg.1 & -1.22617 & 0.003256 & Down \\
\hline TC02004413.hg.1 & -1.215328 & 0.003269 & Down \\
\hline TC02001762.hg.1 & -1.263185 & 0.003271 & Down \\
\hline TC18000587.hg.1 & -1.230616 & 0.003274 & Down \\
\hline TC10001008.hg.1 & -1.241843 & 0.003295 & Down \\
\hline TCOY000340.hg.1 & -1.250366 & 0.003297 & Down \\
\hline TC13000269.hg.1 & -1.260255 & 0.0033 & Down \\
\hline TC20000841.hg.1 & -1.232297 & 0.003318 & Down \\
\hline TC14001908.hg.1 & -1.215891 & 0.003324 & Down \\
\hline TC19000842.hg.1 & -1.258765 & 0.003358 & Down \\
\hline TC04000223.hg.1 & -1.241108 & 0.00336 & Down \\
\hline TC21000697.hg.1 & -1.303591 & 0.003394 & Down \\
\hline TC19000162.hg.1 & -1.200106 & 0.003397 & Down \\
\hline TC10000985.hg.1 & -1.215571 & 0.003402 & Down \\
\hline TC17001640.hg.1 & -1.212141 & 0.003405 & Down \\
\hline TC05000237.hg.1 & -1.373017 & 0.003431 & Down \\
\hline TC09000041.hg.1 & -1.214059 & 0.003441 & Down \\
\hline TC14002178.hg.1 & -1.230783 & 0.003446 & Down \\
\hline TC05002490.hg.1 & -1.232531 & 0.003465 & Down \\
\hline TC15002624.hg.1 & -1.272763 & 0.003483 & Down \\
\hline TC02002012.hg.1 & -1.316727 & 0.003499 & Down \\
\hline TC09000159.hg.1 & -1.390197 & 0.003533 & Down \\
\hline TC09001661.hg.1 & -1.227836 & 0.003577 & Down \\
\hline TC10001055.hg.1 & -1.228079 & 0.003624 & Down \\
\hline TC08001514.hg.1 & -1.277552 & 0.003629 & Down \\
\hline TC11000282.hg.1 & -1.329442 & 0.003642 & Down \\
\hline TC05000068.hg.1 & -1.350724 & 0.003645 & Down \\
\hline TC11001479.hg.1 & -1.279519 & 0.003647 & Down \\
\hline TC18000287.hg.1 & -1.200612 & 0.003655 & Down \\
\hline TCOX000232.hg.1 & -1.202026 & 0.003658 & Down \\
\hline TC01002467.hg.1 & -1.339589 & 0.003689 & Down \\
\hline TC01005508.hg.1 & -1.233888 & 0.003692 & Down \\
\hline TC02000635.hg.1 & -1.204559 & 0.003708 & Down \\
\hline TC05002063.hg.1 & -1.245733 & 0.003747 & Down \\
\hline TCOX002282.hg.1 & -1.216687 & 0.003788 & Down \\
\hline TC06001847.hg.1 & -1.202026 & 0.003791 & Down \\
\hline TC05003245.hg.1 & -1.245568 & 0.003825 & Down \\
\hline TC15002600.hg.1 & -1.202092 & 0.003846 & Down \\
\hline TC05001212.hg.1 & -1.356607 & 0.003867 & Down \\
\hline TC14001340.hg.1 & -1.246169 & 0.003903 & Down \\
\hline TC16002021.hg.1 & -1.243627 & 0.003914 & Down \\
\hline TC19000560.hg.1 & -1.215974 & 0.003922 & Down \\
\hline TC10000041.hg.1 & -1.253239 & 0.003961 & Down \\
\hline TC03002003.hg.1 & -1.293355 & 0.003977 & Down \\
\hline TC01003999.hg.1 & -1.213308 & 0.004016 & Down \\
\hline TC11003279.hg.1 & -1.239214 & 0.004073 & Down \\
\hline TC12000588.hg.1 & -1.255784 & 0.004105 & Down \\
\hline TC03001070.hg.1 & -1.236896 & 0.004107 & Down \\
\hline TC05000876.hg.1 & -1.344018 & 0.004112 & Down \\
\hline
\end{tabular}

Table 2 (continued)

\begin{tabular}{|c|c|c|c|}
\hline Probe set ID & Fold change & p-value & Gene feature \\
\hline TC16001209.hg.1 & -1.206352 & 0.004131 & Down \\
\hline TC22000194.hg.1 & -1.201822 & 0.004141 & Down \\
\hline TC01000906.hg.1 & -1.237207 & 0.004146 & Down \\
\hline TC09001410.hg.1 & -1.328568 & 0.004149 & Down \\
\hline TC15002068.hg.1 & -1.261482 & 0.004152 & Down \\
\hline TC13000345.hg.1 & -1.211536 & 0.004193 & Down \\
\hline TC03002998.hg.1 & -1.271102 & 0.004199 & Down \\
\hline TC03002746.hg.1 & -1.217988 & 0.004201 & Down \\
\hline TC15000264.hg.1 & -1.253176 & 0.004219 & Down \\
\hline TC13000676.hg.1 & -1.259466 & 0.004222 & Down \\
\hline TC13001035.hg.1 & -1.299097 & 0.00423 & Down \\
\hline TC01000516.hg.1 & -1.415817 & 0.004238 & Down \\
\hline TC1 1002367.hg.1 & -1.203564 & 0.004256 & Down \\
\hline TC09001709.hg.1 & -1.365244 & 0.004261 & Down \\
\hline TC10001951.hg.1 & -1.254194 & 0.004269 & Down \\
\hline TC01005957.hg.1 & -1.276394 & 0.00429 & Down \\
\hline TC21000155.hg.1 & -1.253291 & 0.004303 & Down \\
\hline TC08001116.hg.1 & -1.261791 & 0.004358 & Down \\
\hline TC17002050.hg.1 & -1.21877 & 0.004384 & Down \\
\hline TC11001973.hg.1 & -1.210002 & 0.004426 & Down \\
\hline TC10001296.hg.1 & -1.200871 & 0.004441 & Down \\
\hline TC14000289.hg.1 & -1.311616 & 0.004481 & Down \\
\hline TC12000607.hg.1 & -1.30518 & 0.004488 & Down \\
\hline TC05002808.hg.1 & -1.219978 & 0.004507 & Down \\
\hline TC12001404.hg.1 & -1.256127 & 0.004509 & Down \\
\hline TC22001312.hg.1 & -1.204404 & 0.004515 & Down \\
\hline TC13000501.hg.1 & -1.213954 & 0.004517 & Down \\
\hline TC19001712.hg.1 & -1.212198 & 0.004546 & Down \\
\hline TC05001815.hg.1 & -1.239784 & 0.004569 & Down \\
\hline TCOX000283.hg.1 & -1.229291 & 0.004585 & Down \\
\hline TC08001986.hg.1 & -1.281049 & 0.004588 & Down \\
\hline TC14000798.hg.1 & -1.441811 & 0.004598 & Down \\
\hline TC16001481.hg.1 & -1.228168 & 0.004619 & Down \\
\hline TCOX002079.hg.1 & -1.246788 & 0.004622 & Down \\
\hline TC17000557.hg.1 & -1.302083 & 0.004624 & Down \\
\hline TC04002474.hg.1 & -1.274389 & 0.004635 & Down \\
\hline TC17001193.hg.1 & -1.233444 & 0.004648 & Down \\
\hline TC01000331.hg.1 & -1.248432 & 0.004663 & Down \\
\hline TC1 1002966.hg.1 & -1.259327 & 0.004695 & Down \\
\hline TC06002589.hg.1 & -1.216984 & 0.004713 & Down \\
\hline TC06002011.hg.1 & -1.434725 & 0.004731 & Down \\
\hline TCOX000412.hg.1 & -1.250337 & 0.004744 & Down \\
\hline TC14001352.hg.1 & -1.286012 & 0.004755 & Down \\
\hline TC18000425.hg.1 & -1.208537 & 0.004757 & Down \\
\hline TC16002106.hg.1 & -1.246345 & 0.004784 & Down \\
\hline Probe set ID & Fold change & $p$-value & Gene feature \\
\hline \multicolumn{4}{|c|}{$\begin{array}{l}\text { (B) Genes up/down-regulated in non-purified lung } T C S^{S V 40} \text { compared with purified } \\
\text { primary lung TCS }\end{array}$} \\
\hline TC07002463.hg.1 & 2.512854 & $2.70 \mathrm{E}-05$ & Up \\
\hline
\end{tabular}


Table 2 (continued)

\begin{tabular}{|c|c|c|c|}
\hline Probe set ID & Fold change & $p$-value & Gene feature \\
\hline TCOM000020.hg.1 & 5.689014 & $3.00 \mathrm{E}-05$ & Up \\
\hline TC03003114.hg.1 & 4.799932 & $3.20 E-05$ & Up \\
\hline TC16000648.hg.1 & -2.174193 & $3.50 E-05$ & Down \\
\hline TC17001801.hg.1 & -2.453481 & $3.80 E-05$ & Down \\
\hline TC02003744.hg.1 & 1.994216 & $4.00 \mathrm{E}-05$ & Up \\
\hline TC11002894.hg.1 & 4.163733 & 4.30E-05 & Up \\
\hline TC11003109.hg.1 & 5.91745 & 4.50E-05 & Up \\
\hline TC20000952.hg.1 & -2.038322 & 4.80E-05 & Down \\
\hline TC12001286.hg.1 & -1.75483 & $5.10 \mathrm{E}-05$ & Down \\
\hline TC09000971.hg.1 & -1.873134 & $5.30 \mathrm{E}-05$ & Down \\
\hline TC02002456.hg.1 & -1.779116 & $5.60 \mathrm{E}-05$ & Down \\
\hline TC04002560.hg.1 & 2.777915 & $5.80 \mathrm{E}-05$ & Up \\
\hline TC05003313.hg.1 & 2.480494 & $6.10 E-05$ & Up \\
\hline TC02000205.hg.1 & -1.822189 & $6.40 \mathrm{E}-05$ & Down \\
\hline TC08002510.hg.1 & 2.45585 & $6.60 \mathrm{E}-05$ & Up \\
\hline TC20000045.hg.1 & -2.011887 & $6.90 \mathrm{E}-05$ & Down \\
\hline TC12000927.hg.1 & -2.097234 & $7.10 \mathrm{E}-05$ & Down \\
\hline TC09001648.hg.1 & -1.962423 & 7.40E-05 & Down \\
\hline TCOX000985.hg.1 & -2.143818 & $7.70 \mathrm{E}-05$ & Down \\
\hline TC01003308.hg.1 & -1.928676 & 7.90E-05 & Down \\
\hline TC22000156.hg.1 & 1.958661 & $8.20 \mathrm{E}-05$ & Up \\
\hline TC04001945.hg.1 & -2.062837 & 8.40E-05 & Down \\
\hline TC06000639.hg.1 & -2.085546 & $8.70 \mathrm{E}-05$ & Down \\
\hline TC06001978.hg.1 & -1.915437 & $9.00 \mathrm{E}-05$ & Down \\
\hline TC15000452.hg.1 & -1.936021 & $9.20 \mathrm{E}-05$ & Down \\
\hline TC02001940.hg.1 & -1.951298 & $9.50 \mathrm{E}-05$ & Down \\
\hline TC06000568.hg.1 & -1.714082 & $9.70 \mathrm{E}-05$ & Down \\
\hline TC08000302.hg.1 & -1.960068 & 1.00E-04 & Down \\
\hline TC17002479.hg.1 & 1.736394 & 0.000102 & Up \\
\hline TC04002890.hg.1 & 2.211564 & 0.000105 & Up \\
\hline TC20000650.hg.1 & -1.762113 & 0.000108 & Down \\
\hline TC02004814.hg.1 & -1.74863 & 0.00011 & Down \\
\hline TC17001773.hg.1 & -1.57464 & 0.000113 & Down \\
\hline TC07001784.hg.1 & -1.815099 & 0.000115 & Down \\
\hline TC05001593.hg.1 & -1.890235 & 0.000118 & Down \\
\hline TCOX001889.hg.1 & 1.807993 & 0.000121 & Up \\
\hline TC1 1002382.hg.1 & -2.886542 & 0.000123 & Down \\
\hline TCOM000022.hg.1 & 2.047915 & 0.000126 & Up \\
\hline TC06004042.hg.1 & -1.606168 & 0.000128 & Down \\
\hline TC12001449.hg.1 & -1.770781 & 0.000131 & Down \\
\hline TC09000096.hg.1 & -1.882465 & 0.000134 & Down \\
\hline TCOX001824.hg.1 & 1.668798 & 0.000136 & Up \\
\hline TC08000801.hg.1 & -1.742013 & 0.000139 & Down \\
\hline TC01005811.hg.1 & 1.59682 & 0.000141 & Up \\
\hline TCOX001410.hg.1 & -1.540303 & 0.000144 & Down \\
\hline TC13000783.hg.1 & -2.094361 & 0.000147 & Down \\
\hline TCOX001411.hg.1 & -1.589254 & 0.000149 & Down \\
\hline TC07000702.hg.1 & -1.788577 & 0.000152 & Down \\
\hline TC07002531.hg.1 & 1.762967 & 0.000154 & Up \\
\hline TC20000373.hg.1 & -1.578384 & 0.000157 & Down \\
\hline TC06002402.hg.1 & 1.778454 & 0.00016 & Up \\
\hline TC09001483.hg.1 & 1.350997 & 0.000162 & Up \\
\hline TC07001655.hg.1 & -1.528388 & 0.000165 & Down \\
\hline
\end{tabular}

Table 2 (continued)

\begin{tabular}{|c|c|c|c|}
\hline Probe set ID & Fold change & $p$-value & Gene feature \\
\hline TC01004012.hg.1 & 1.503918 & 0.000167 & Up \\
\hline TCOX001136.hg.1 & -1.534991 & 0.00017 & Down \\
\hline TC01002339.hg.1 & -1.538502 & 0.000173 & Down \\
\hline TC12000633.hg.1 & -1.841917 & 0.000175 & Down \\
\hline TC02001602.hg.1 & -1.669996 & 0.000178 & Down \\
\hline TC16001991.hg.1 & -1.351113 & 0.00018 & Down \\
\hline TC03001841.hg.1 & -1.638782 & 0.000183 & Down \\
\hline TC22001175.hg.1 & -1.558447 & 0.000186 & Down \\
\hline TC14001892.hg.1 & -1.603015 & 0.000188 & Down \\
\hline TC08002500.hg.1 & -1.406478 & 0.000191 & Down \\
\hline TC02004615.hg.1 & 1.498343 & 0.000193 & Up \\
\hline TC12000231.hg.1 & -1.476476 & 0.000196 & Down \\
\hline TC14000711.hg.1 & -1.357936 & 0.000198 & Down \\
\hline TC16001289.hg.1 & -1.683139 & 0.000201 & Down \\
\hline TC0X001822.hg.1 & 1.466789 & 0.000204 & Up \\
\hline TC02000396.hg.1 & -1.782594 & 0.000206 & Down \\
\hline TC15002247.hg.1 & 1.443916 & 0.000209 & Up \\
\hline TC10000686.hg.1 & -1.508486 & 0.000211 & Down \\
\hline TC22000400.hg.1 & -1.524343 & 0.000214 & Down \\
\hline TC06003416.hg.1 & -1.409684 & 0.000217 & Down \\
\hline TC16002030.hg.1 & -1.497175 & 0.000219 & Down \\
\hline TC01002976.hg.1 & -1.555849 & 0.000222 & Down \\
\hline TC15001326.hg.1 & -1.510761 & 0.000224 & Down \\
\hline TC07002052.hg.1 & -1.585855 & 0.000227 & Down \\
\hline TC12001995.hg.1 & -1.471839 & 0.00023 & Down \\
\hline TC19000163.hg.1 & -1.563519 & 0.000232 & Down \\
\hline TC21000931.hg.1 & -1.527689 & 0.000235 & Down \\
\hline TC02003743.hg.1 & 1.519001 & 0.000237 & Up \\
\hline TC10000866.hg.1 & -1.606186 & 0.00024 & Down \\
\hline TC04002346.hg.1 & 1.48682 & 0.000243 & Up \\
\hline TC08001887.hg.1 & 1.336453 & 0.000245 & Up \\
\hline TC06001651.hg.1 & -1.472924 & 0.000248 & Down \\
\hline TC04000811.hg.1 & -1.415531 & 0.00025 & Down \\
\hline TC0X000279.hg.1 & -1.52073 & 0.000253 & Down \\
\hline TC04001635.hg.1 & -2.149662 & 0.000256 & Down \\
\hline TC19000767.hg.1 & -1.792384 & 0.000258 & Down \\
\hline TC04000160.hg.1 & -1.443667 & 0.000261 & Down \\
\hline TC16001498.hg.1 & 1.716487 & 0.000263 & Up \\
\hline TC04001524.hg.1 & -1.497583 & 0.000266 & Down \\
\hline TC01002798.hg.1 & -1.728642 & 0.000269 & Down \\
\hline TC04002187.hg.1 & 1.535246 & 0.000271 & Up \\
\hline TC05002063.hg.1 & -1.378512 & 0.000274 & Down \\
\hline TC09002847.hg.1 & -1.569396 & 0.000276 & Down \\
\hline TC05002818.hg.1 & -1.548005 & 0.000279 & Down \\
\hline TCOX001292.hg.1 & -2.249923 & 0.000282 & Down \\
\hline TCOX000450.hg.1 & -1.560574 & 0.000284 & Down \\
\hline TC03001056.hg.1 & -1.589272 & 0.000287 & Down \\
\hline TC09001898.hg.1 & -1.413369 & 0.000289 & Down \\
\hline TC02000955.hg.1 & -1.593632 & 0.000292 & Down \\
\hline TC02001432.hg.1 & -1.603841 & 0.000294 & Down \\
\hline TC03000195.hg.1 & -1.56613 & 0.000297 & Down \\
\hline TC01002877.hg.1 & 1.393011 & $3.00 E-04$ & Up \\
\hline TC02001366.hg.1 & -1.50307 & 0.000302 & Down \\
\hline
\end{tabular}


Table 2 (continued)

\begin{tabular}{|c|c|c|c|}
\hline Probe set ID & Fold change & $p$-value & Gene feature \\
\hline TC06001365.hg.1 & -1.798942 & 0.000305 & Down \\
\hline TC21000483.hg.1 & -1.526505 & 0.000307 & Down \\
\hline TC1 1002486.hg.1 & -1.571098 & 0.00031 & Down \\
\hline TC10002723.hg.1 & -1.737066 & 0.000313 & Down \\
\hline TC09002408.hg.1 & 2.424384 & 0.000315 & Up \\
\hline TC02002994.hg.1 & -1.689071 & 0.000318 & Down \\
\hline TC01000642.hg.1 & -1.53535 & 0.00032 & Down \\
\hline TC14000620.hg.1 & -1.463791 & 0.000323 & Down \\
\hline TC07003059.hg.1 & 1.529988 & 0.000326 & Up \\
\hline TC21000650.hg.1 & -1.430525 & 0.000328 & Down \\
\hline TC11001010.hg.1 & -1.579571 & 0.000331 & Down \\
\hline TC05000526.hg.1 & -1.395094 & 0.000333 & Down \\
\hline TC09001461.hg.1 & -1.680036 & 0.000336 & Down \\
\hline TC09002258.hg.1 & 1.60311 & 0.000339 & Up \\
\hline TC07001656.hg.1 & -1.511784 & 0.000341 & Down \\
\hline TC10000439.hg.1 & -1.472641 & 0.000344 & Down \\
\hline TC05002279.hg.1 & -1.557636 & 0.000346 & Down \\
\hline TC14000775.hg.1 & 1.356138 & 0.000349 & Up \\
\hline TC14002059.hg.1 & 1.523057 & 0.000352 & Up \\
\hline TC15001184.hg.1 & -1.391194 & 0.000357 & Down \\
\hline TC11003147.hg.1 & -1.401112 & 0.000359 & Down \\
\hline TC07002742.hg.1 & -1.399246 & 0.000362 & Down \\
\hline TC08001864.hg.1 & 2.950612 & 0.000365 & Up \\
\hline TC09001602.hg.1 & -1.880185 & 0.000367 & Down \\
\hline TC05002800.hg.1 & -1.563391 & 0.00037 & Down \\
\hline TC11002476.hg.1 & -1.690386 & 0.000372 & Down \\
\hline TC07002321.hg.1 & 1.27127 & 0.000375 & Up \\
\hline TC01002633.hg.1 & -1.686971 & 0.000378 & Down \\
\hline TC05002022.hg.1 & 1.497479 & 0.00038 & Up \\
\hline TC17000728.hg.1 & -1.965686 & 0.000383 & Down \\
\hline TC10002300.hg.1 & 1.994904 & 0.000385 & Up \\
\hline TC01002746.hg.1 & -1.430809 & 0.000388 & Down \\
\hline TC05000708.hg.1 & -1.617304 & 0.000391 & Down \\
\hline TC17000491.hg.1 & -1.515315 & 0.000396 & Down \\
\hline TC09001534.hg.1 & -1.459227 & 0.000398 & Down \\
\hline TC12003194.hg.1 & -1.49803 & 0.000401 & Down \\
\hline TC12002688.hg.1 & -1.685657 & 0.000403 & Down \\
\hline TC04000508.hg.1 & -1.50861 & 0.000406 & Down \\
\hline TC01002620.hg.1 & -1.500063 & 0.000409 & Down \\
\hline TC21000887.hg.1 & 1.383973 & 0.000414 & Up \\
\hline TC18000603.hg.1 & 2.095404 & 0.000416 & Up \\
\hline TC02002711.hg.1 & -1.374202 & 0.000419 & Down \\
\hline TC01004110.hg.1 & 1.321687 & 0.000422 & Up \\
\hline TC06000824.hg.1 & -1.53592 & 0.000427 & Down \\
\hline TC01004763.hg.1 & 1.590669 & 0.000429 & Up \\
\hline TC12000558.hg.1 & -1.427752 & 0.000432 & Down \\
\hline TC20001202.hg.1 & 1.308112 & 0.000435 & Up \\
\hline TC07001267.hg.1 & -1.48023 & 0.000437 & Down \\
\hline TC06004031.hg.1 & -1.480003 & 0.00044 & Down \\
\hline TC22001053.hg.1 & -1.545286 & 0.000442 & Down \\
\hline TCOX001158.hg.1 & -1.890718 & 0.000445 & Down \\
\hline TC16002013.hg.1 & -1.631206 & 0.000448 & Down \\
\hline TC04002642.hg.1 & -1.43066 & 0.000453 & Down \\
\hline
\end{tabular}

Table 2 (continued)

\begin{tabular}{|c|c|c|c|}
\hline Probe set ID & Fold change & $p$-value & Gene feature \\
\hline TC21000042.hg.1 & 2.453488 & 0.000455 & Up \\
\hline TC20001679.hg.1 & 1.762017 & 0.000458 & Up \\
\hline TC14001143.hg.1 & -1.26258 & 0.000461 & Down \\
\hline TC11002810.hg.1 & 1.384015 & 0.000463 & Up \\
\hline TC11001848.hg.1 & 1.462057 & 0.000466 & Up \\
\hline TC12003094.hg.1 & -1.4237 & 0.000468 & Down \\
\hline TC11002492.hg.1 & -1.316801 & 0.000471 & Down \\
\hline TC04000429.hg.1 & 1.289905 & 0.000474 & Up \\
\hline TC01005268.hg.1 & 2.055799 & 0.000476 & Up \\
\hline TC01000487.hg.1 & 1.354288 & 0.000479 & Up \\
\hline TC02001429.hg.1 & -1.455529 & 0.000487 & Down \\
\hline TC19001967.hg.1 & 1.548221 & 0.000489 & Up \\
\hline TC12000813.hg.1 & -1.316062 & 0.000492 & Down \\
\hline TC22001417.hg.1 & -1.597844 & 0.000494 & Down \\
\hline TC08000652.hg.1 & -1.574349 & 0.000497 & Down \\
\hline TC18000408.hg.1 & 1.572603 & 0.000499 & Up \\
\hline TC11003186.hg.1 & 1.33033 & 0.000502 & Up \\
\hline TC01004251.hg.1 & 1.32401 & 0.000505 & Up \\
\hline TC08002253.hg.1 & -2.279339 & 0.000507 & Down \\
\hline TC01002594.hg.1 & -1.779567 & 0.00051 & Down \\
\hline TC03003057.hg.1 & 1.407168 & 0.000512 & Up \\
\hline TC0X002258.hg.1 & -1.448026 & 0.000515 & Down \\
\hline TC14001731.hg.1 & 1.360459 & 0.000518 & Up \\
\hline TC11000031.hg.1 & -1.412204 & 0.00052 & Down \\
\hline TC08001833.hg.1 & 1.37955 & 0.000525 & Up \\
\hline TC03001342.hg.1 & -1.290438 & 0.000528 & Down \\
\hline TC17002307.hg.1 & 1.569429 & 0.000531 & Up \\
\hline TC04002813.hg.1 & 1.2442 & 0.000533 & Up \\
\hline TC05000688.hg.1 & -1.475197 & 0.000536 & Down \\
\hline TC05002034.hg.1 & -1.389715 & 0.000538 & Down \\
\hline TC12000088.hg.1 & 1.466129 & 0.000544 & Up \\
\hline TC05001142.hg.1 & -1.347908 & 0.000546 & Down \\
\hline TC06002303.hg.1 & -1.437374 & 0.000562 & Down \\
\hline TC09000586.hg.1 & -1.463022 & 0.000564 & Down \\
\hline TC15001796.hg.1 & -1.33117 & 0.000567 & Down \\
\hline TC17001193.hg.1 & -1.355381 & 0.00057 & Down \\
\hline TC06002340.hg.1 & -1.4849 & 0.000575 & Down \\
\hline TC05003112.hg.1 & 1.638048 & 0.000577 & Up \\
\hline TC06000705.hg.1 & -1.607894 & 0.000583 & Down \\
\hline TC12000238.hg.1 & -1.335439 & 0.000585 & Down \\
\hline TC01000565.hg.1 & -1.337671 & 0.000588 & Down \\
\hline TC02000092.hg.1 & -1.330873 & 0.00059 & Down \\
\hline TCOX002282.hg.1 & -1.325366 & 0.000593 & Down \\
\hline TC09000383.hg.1 & -1.274316 & 0.000598 & Down \\
\hline TC11003210.hg.1 & 1.231344 & 0.000601 & Up \\
\hline TC15001604.hg.1 & -1.487855 & 0.000603 & Down \\
\hline TC10002919.hg.1 & -1.480823 & 0.000606 & Down \\
\hline TC21000511.hg.1 & 1.377046 & 0.000611 & Up \\
\hline TC02003970.hg.1 & -1.387345 & 0.000616 & Down \\
\hline TC07002506.hg.1 & -1.369026 & 0.000619 & Down \\
\hline TC01003622.hg.1 & 1.356757 & 0.000621 & Up \\
\hline TC12001052.hg.1 & -1.676926 & 0.000624 & Down \\
\hline TC13000706.hg.1 & -1.254769 & 0.000627 & Down \\
\hline
\end{tabular}


Table 2 (continued)

\begin{tabular}{|c|c|c|c|}
\hline Probe set ID & Fold change & $p$-value & Gene feature \\
\hline TC01004698.hg.1 & 1.469743 & 0.000632 & Up \\
\hline TC07001706.hg.1 & -1.439366 & 0.000636 & Down \\
\hline TC07001709.hg.1 & -1.439366 & 0.000636 & Down \\
\hline TC05001595.hg.1 & -1.447738 & 0.00064 & Down \\
\hline TC02001302.hg.1 & -1.576693 & 0.000642 & Down \\
\hline TC05001591.hg.1 & -1.203418 & 0.000645 & Down \\
\hline TC08002317.hg.1 & -1.330718 & 0.000647 & Down \\
\hline TC09000516.hg.1 & -1.664198 & 0.00065 & Down \\
\hline TC12002858.hg.1 & 1.350881 & 0.000655 & Up \\
\hline TC01005360.hg.1 & 1.271483 & 0.000658 & Up \\
\hline TC19001567.hg.1 & 1.505097 & 0.00066 & Up \\
\hline TC07000690.hg.1 & -1.411787 & 0.000663 & Down \\
\hline TC03001571.hg.1 & -1.477672 & 0.000666 & Down \\
\hline TC06002795.hg.1 & 1.436937 & 0.000668 & Up \\
\hline TC06003874.hg.1 & -1.29272 & 0.000671 & Down \\
\hline TC03002116.hg.1 & -1.412674 & 0.000673 & Down \\
\hline TC05000127.hg.1 & 1.513078 & 0.000676 & Up \\
\hline TC17002638.hg.1 & 1.348987 & 0.000679 & Up \\
\hline TC20000751.hg.1 & -1.402838 & 0.000681 & Down \\
\hline TC08002421.hg.1 & -1.74914 & 0.000684 & Down \\
\hline TC01005354.hg.1 & 1.229747 & 0.000686 & Up \\
\hline TC12003088.hg.1 & -1.281487 & 0.000689 & Down \\
\hline TC08001583.hg.1 & -1.537328 & 0.000691 & Down \\
\hline TC05000998.hg.1 & -1.483305 & 0.000694 & Down \\
\hline TC04001473.hg.1 & -1.432026 & 0.000697 & Down \\
\hline TC04002443.hg.1 & -1.487139 & 0.000702 & Down \\
\hline TC02000005.hg.1 & -1.472423 & 0.000704 & Down \\
\hline TC0X001624.hg.1 & -1.596058 & 0.000707 & Down \\
\hline TC04000461.hg.1 & 1.412843 & 0.00071 & Up \\
\hline TC08000450.hg.1 & -1.615791 & 0.000712 & Down \\
\hline TC18000833.hg.1 & 1.283471 & 0.000715 & Up \\
\hline TC01003671.hg.1 & -1.422938 & 0.00072 & Down \\
\hline TC01001291.hg.1 & 1.409687 & 0.000723 & Up \\
\hline TC10001432.hg.1 & -1.432311 & 0.000725 & Down \\
\hline TC17001310.hg.1 & -1.283493 & 0.000728 & Down \\
\hline TC08002093.hg.1 & -1.29301 & 0.00073 & Down \\
\hline TC20001234.hg.1 & 1.431939 & 0.000733 & Up \\
\hline TC1 1000270.hg.1 & 1.380022 & 0.000736 & Up \\
\hline TC06002451.hg.1 & -1.361116 & 0.000738 & Down \\
\hline TC02003138.hg.1 & 1.349475 & 0.000741 & Up \\
\hline TC05000183.hg.1 & -1.48138 & 0.000746 & Down \\
\hline TC01003990.hg.1 & -1.390426 & 0.000749 & Down \\
\hline TC02001762.hg.1 & -1.302759 & 0.000756 & Down \\
\hline TCOX001430.hg.1 & -1.470658 & 0.000762 & Down \\
\hline TC05001244.hg.1 & 1.28468 & 0.000764 & Up \\
\hline TC16001854.hg.1 & -1.274852 & 0.000767 & Down \\
\hline TC19000179.hg.1 & -1.220968 & 0.000769 & Down \\
\hline TC01005416.hg.1 & 1.308645 & 0.000772 & Up \\
\hline TC21000041.hg.1 & 1.720569 & 0.000777 & Up \\
\hline TC06002615.hg.1 & 1.698567 & 0.000782 & Up \\
\hline TCOX001210.hg.1 & -1.72284 & 0.000787 & Down \\
\hline TC08000385.hg.1 & -1.30756 & 0.00079 & Down \\
\hline TC01001501.hg.1 & -1.261062 & 0.000793 & Down \\
\hline
\end{tabular}

Table 2 (continued)

\begin{tabular}{|c|c|c|c|}
\hline Probe set ID & Fold change & $p$-value & Gene feature \\
\hline TC11000882.hg.1 & 1.360165 & 0.000795 & Up \\
\hline TC17002123.hg.1 & 1.252373 & 0.000798 & Up \\
\hline TC10001266.hg.1 & -1.270974 & $8.00 E-04$ & Down \\
\hline TC04000223.hg.1 & -1.399388 & 0.000803 & Down \\
\hline TC02004784.hg.1 & -1.270101 & 0.000806 & Down \\
\hline TC03003257.hg.1 & -1.473539 & 0.000811 & Down \\
\hline TC11002784.hg.1 & 1.544173 & 0.000816 & Up \\
\hline TC17000637.hg.1 & 1.549618 & 0.000821 & Up \\
\hline TC21000447.hg.1 & -1.357028 & 0.000824 & Down \\
\hline TC16001066.hg.1 & -1.250451 & 0.000826 & Down \\
\hline TC10001922.hg.1 & 1.308392 & 0.000834 & Up \\
\hline TC11002991.hg.1 & -1.203628 & 0.000839 & Down \\
\hline TC03003014.hg.1 & -1.272182 & 0.000845 & Down \\
\hline TC05001291.hg.1 & -1.22311 & 0.000847 & Down \\
\hline TC11002432.hg.1 & -1.294734 & 0.00085 & Down \\
\hline TC07000282.hg.1 & -1.386049 & 0.000852 & Down \\
\hline TCOX001907.hg.1 & 1.514463 & 0.000855 & Up \\
\hline TC12000589.hg.1 & -1.437338 & 0.000858 & Down \\
\hline TC01001111.hg.1 & 1.562931 & 0.00086 & Up \\
\hline TC08000734.hg.1 & -1.2404 & 0.000863 & Down \\
\hline TC0X000649.hg.1 & -1.204547 & 0.000865 & Down \\
\hline TC12000005.hg.1 & -1.230721 & 0.000868 & Down \\
\hline TC06003061.hg.1 & 1.681888 & 0.000871 & Up \\
\hline TC11000655.hg.1 & -1.343701 & 0.000873 & Down \\
\hline TC09000963.hg.1 & -1.43499 & 0.000881 & Down \\
\hline TC15002417.hg.1 & -1.286319 & 0.000883 & Down \\
\hline TC07003181.hg.1 & -1.334931 & 0.000886 & Down \\
\hline TC10001115.hg.1 & 1.283726 & 0.000889 & Up \\
\hline TC12000578.hg.1 & 1.240881 & 0.000891 & Up \\
\hline TC01005437.hg.1 & 1.248673 & 0.000894 & Up \\
\hline TC17002178.hg.1 & -1.402437 & 0.000896 & Down \\
\hline TC14001936.hg.1 & 1.238815 & 0.000899 & Up \\
\hline TC14000725.hg.1 & -1.30455 & 0.000904 & Down \\
\hline TC13000186.hg.1 & -1.264527 & 0.000907 & Down \\
\hline TC05002042.hg.1 & -1.348682 & 0.000909 & Down \\
\hline TC12001770.hg.1 & 1.739266 & 0.000917 & Up \\
\hline TC17001656.hg.1 & -1.467195 & 0.000922 & Down \\
\hline TC01005905.hg.1 & -1.214045 & 0.000925 & Down \\
\hline TCOY000302.hg.1 & -1.280838 & 0.000931 & Down \\
\hline TCOY000304.hg.1 & -1.280838 & 0.000931 & Down \\
\hline TC02004706.hg.1 & 1.367562 & 0.000935 & Up \\
\hline TC06003540.hg.1 & 1.286007 & 0.000938 & Up \\
\hline TC12001099.hg.1 & -1.215286 & 0.000941 & Down \\
\hline TC10000843.hg.1 & 1.313133 & 0.000943 & Up \\
\hline TC03001294.hg.1 & 1.437371 & 0.000946 & Up \\
\hline TC15000406.hg.1 & -1.277322 & 0.000948 & Down \\
\hline TC05002576.hg.1 & -1.39417 & 0.000951 & Down \\
\hline TC14000991.hg.1 & 1.299653 & 0.000954 & Up \\
\hline TC02003574.hg.1 & -1.248912 & 0.000956 & Down \\
\hline TC15002494.hg.1 & -1.335358 & 0.000959 & Down \\
\hline TC10001764.hg.1 & -1.280423 & 0.000961 & Down \\
\hline TC02001836.hg.1 & -1.445879 & 0.000964 & Down \\
\hline TC06000102.hg.1 & -1.497939 & 0.000969 & Down \\
\hline
\end{tabular}


Table 2 (continued)

\begin{tabular}{|c|c|c|c|}
\hline Probe set ID & Fold change & $p$-value & Gene feature \\
\hline TC03001531.hg.1 & 1.252613 & 0.000972 & Up \\
\hline TC08001082.hg.1 & -1.277646 & 0.000977 & Down \\
\hline TC03000629.hg.1 & -1.58654 & 0.00098 & Down \\
\hline TC04000854.hg.1 & -1.369976 & 0.000982 & Down \\
\hline TCOX000283.hg.1 & -1.606025 & 0.000987 & Down \\
\hline TC02003376.hg.1 & 1.288982 & 0.00099 & Up \\
\hline TC16001973.hg.1 & 1.416974 & 0.000995 & Up \\
\hline TC09001936.hg.1 & -1.55743 & 0.000998 & Down \\
\hline TC14002181.hg.1 & -1.292272 & 0.001 & Down \\
\hline TC19002169.hg.1 & 1.292553 & 0.001003 & Up \\
\hline TC22001135.hg.1 & -1.237744 & 0.001008 & Down \\
\hline TC14001911.hg.1 & 1.226465 & 0.001013 & Up \\
\hline TC04001384.hg.1 & 1.326226 & 0.001016 & Up \\
\hline TC09002810.hg.1 & -1.253762 & 0.001018 & Down \\
\hline TC01006061.hg.1 & 1.239967 & 0.001024 & Up \\
\hline TC09002176.hg.1 & -1.252655 & 0.001026 & Down \\
\hline TCOX001762.hg.1 & -1.338176 & 0.001029 & Down \\
\hline TC07000339.hg.1 & -1.441623 & 0.001034 & Down \\
\hline TC18000715.hg.1 & -1.339535 & 0.001039 & Down \\
\hline TC20001391.hg.1 & 1.279887 & 0.001042 & Up \\
\hline TC04001218.hg.1 & 1.463061 & 0.001044 & Up \\
\hline TC01005893.hg.1 & -1.235444 & 0.001047 & Down \\
\hline TC03001845.hg.1 & 1.468478 & 0.001052 & Up \\
\hline TC01006068.hg.1 & -1.684105 & 0.001055 & Down \\
\hline TC20000564.hg.1 & -1.33301 & 0.001057 & Down \\
\hline TC20000106.hg.1 & 1.445871 & 0.00106 & Up \\
\hline TC04000543.hg.1 & 1.606432 & 0.001063 & Up \\
\hline TC12000805.hg.1 & -1.359918 & 0.001065 & Down \\
\hline TC04001743.hg.1 & 1.217508 & 0.001068 & Up \\
\hline TC09000060.hg.1 & -1.302308 & 0.00107 & Down \\
\hline TC03001672.hg.1 & -1.340418 & 0.001073 & Down \\
\hline TC08002335.hg.1 & -1.675618 & 0.001076 & Down \\
\hline TC09001709.hg.1 & -1.550874 & 0.001078 & Down \\
\hline TC21000663.hg.1 & 1.304123 & 0.001081 & Up \\
\hline TC08002008.hg.1 & 1.368127 & 0.001083 & Up \\
\hline TC20000053.hg.1 & -1.230537 & 0.001088 & Down \\
\hline TC01003510.hg.1 & -1.353839 & 0.001091 & Down \\
\hline TC11002378.hg.1 & 1.452002 & 0.001094 & Up \\
\hline TC03001943.hg.1 & -1.331955 & 0.001099 & Down \\
\hline TC05002812.hg.1 & -1.25135 & 0.001101 & Down \\
\hline TC03001366.hg.1 & -1.383993 & 0.001104 & Down \\
\hline TC01002044.hg.1 & 1.389355 & 0.001107 & Up \\
\hline TC03002276.hg.1 & -1.385647 & 0.001109 & Down \\
\hline TC12000315.hg.1 & -1.378694 & 0.001112 & Down \\
\hline TC02000413.hg.1 & -1.31176 & 0.001114 & Down \\
\hline TC08002504.hg.1 & -1.386422 & 0.001117 & Down \\
\hline TC01001142.hg.1 & 1.505991 & 0.001127 & Up \\
\hline TC07001801.hg.1 & 1.753925 & 0.00113 & Up \\
\hline TC05002870.hg.1 & 1.302868 & 0.001133 & Up \\
\hline TC02002904.hg.1 & -1.387138 & 0.001135 & Down \\
\hline TC11001108.hg.1 & 1.530186 & 0.00114 & Up \\
\hline TC13001025.hg.1 & 1.287199 & 0.001146 & Up \\
\hline TC09001939.hg.1 & -1.354181 & 0.001148 & Down \\
\hline
\end{tabular}

Table 2 (continued)

\begin{tabular}{|c|c|c|c|}
\hline Probe set ID & Fold change & $p$-value & Gene feature \\
\hline TC19000963.hg.1 & 1.281426 & 0.001156 & Up \\
\hline TC14000314.hg.1 & 1.326006 & 0.001159 & Up \\
\hline TC06002689.hg.1 & 1.339061 & 0.001161 & Up \\
\hline TC11000768.hg.1 & -1.28868 & 0.001164 & Down \\
\hline TC12000107.hg.1 & 1.674608 & 0.001166 & Up \\
\hline TC07002087.hg.1 & -1.291715 & 0.001172 & Down \\
\hline TC02003914.hg.1 & -1.506351 & 0.001174 & Down \\
\hline TC11002142.hg.1 & -1.78159 & 0.001177 & Down \\
\hline TC01002397.hg.1 & -1.278246 & 0.001179 & Down \\
\hline TC19000409.hg.1 & -1.323654 & 0.001182 & Down \\
\hline TC02001844.hg.1 & 1.297798 & 0.001187 & Up \\
\hline TC07001099.hg.1 & -1.298125 & 0.00119 & Down \\
\hline TC01004750.hg.1 & -1.393313 & 0.001192 & Down \\
\hline TC05001001.hg.1 & -1.289514 & 0.001195 & Down \\
\hline TC14001735.hg.1 & 1.217484 & 0.0012 & Up \\
\hline TC02004275.hg.1 & -1.283611 & 0.001203 & Down \\
\hline TC08001850.hg.1 & 1.293788 & 0.001205 & Up \\
\hline TC09000043.hg.1 & 1.326023 & 0.001208 & Up \\
\hline TC02000138.hg.1 & 1.378171 & 0.00121 & Up \\
\hline TC0X002006.hg.1 & -1.254125 & 0.001213 & Down \\
\hline TC05001174.hg.1 & 1.248122 & 0.001216 & Up \\
\hline TC18000381.hg.1 & -1.439065 & 0.001218 & Down \\
\hline TCOX002099.hg.1 & 1.697539 & 0.001221 & Up \\
\hline TC15001301.hg.1 & 1.38106 & 0.001223 & Up \\
\hline TC01000109.hg.1 & 1.392654 & 0.001231 & Up \\
\hline TCOX001298.hg.1 & 1.461986 & 0.001234 & Up \\
\hline TCOX001901.hg.1 & -1.233679 & 0.001239 & Down \\
\hline TC04002100.hg.1 & -1.359631 & 0.001244 & Down \\
\hline TC20000460.hg.1 & -1.318744 & 0.001247 & Down \\
\hline TC0X000064.hg.1 & 1.391058 & 0.001252 & Up \\
\hline TCOX001094.hg.1 & -1.429617 & 0.001257 & Down \\
\hline TC11002051.hg.1 & -1.484736 & 0.00126 & Down \\
\hline TC12001929.hg.1 & -1.342651 & 0.001265 & Down \\
\hline TC11003367.hg.1 & 1.656682 & 0.001268 & Up \\
\hline TC02003380.hg.1 & 1.301934 & 0.001275 & Up \\
\hline TC21000762.hg.1 & 1.361985 & 0.001278 & Up \\
\hline TCOM000023.hg.1 & 1.382876 & 0.00128 & Up \\
\hline TC02004390.hg.1 & 1.446827 & 0.001283 & Up \\
\hline TC10002633.hg.1 & -1.301706 & 0.001288 & Down \\
\hline TC01006076.hg.1 & -1.245012 & 0.001291 & Down \\
\hline TC12002203.hg.1 & 1.526303 & 0.001296 & Up \\
\hline TC11002740.hg.1 & -1.283389 & 0.001299 & Down \\
\hline TC04002160.hg.1 & -1.262713 & 0.001301 & Down \\
\hline TC17002633.hg.1 & 1.378591 & 0.001304 & Up \\
\hline TC12001768.hg.1 & -1.398601 & 0.001306 & Down \\
\hline TC04002095.hg.1 & 1.241208 & 0.001312 & Up \\
\hline TC05001178.hg.1 & 1.339261 & 0.001317 & Up \\
\hline TC04001688.hg.1 & 1.362728 & 0.001319 & Up \\
\hline TC10002901.hg.1 & -1.278397 & 0.001322 & Down \\
\hline TC15001808.hg.1 & 1.434852 & 0.00133 & Up \\
\hline TC04000710.hg.1 & 1.355916 & 0.001332 & Up \\
\hline TC10002092.hg.1 & 1.281193 & 0.001335 & Up \\
\hline TC09002305.hg.1 & 1.26724 & 0.001343 & Up \\
\hline
\end{tabular}


Table 2 (continued)

\begin{tabular}{|c|c|c|c|}
\hline Probe set ID & Fold change & $p$-value & Gene feature \\
\hline TC12000651.hg.1 & -1.351627 & 0.001345 & Down \\
\hline TC10001511.hg.1 & -1.316949 & 0.001356 & Down \\
\hline TC01000472.hg.1 & -1.298179 & 0.001358 & Down \\
\hline TC1 1003246.hg.1 & 1.335547 & 0.001366 & Up \\
\hline TC1 1000161.hg.1 & -1.27089 & 0.001374 & Down \\
\hline TC12000359.hg.1 & 1.237241 & 0.001379 & Up \\
\hline TC12001702.hg.1 & -1.275897 & 0.001382 & Down \\
\hline TC08001540.hg.1 & -1.346581 & 0.001384 & Down \\
\hline TC08001886.hg.1 & 1.259731 & 0.001387 & Up \\
\hline TC13000269.hg.1 & -1.424301 & 0.001389 & Down \\
\hline TC01005067.hg.1 & 1.487055 & 0.001392 & Up \\
\hline TC01003976.hg.1 & 1.273839 & 0.001395 & Up \\
\hline TC10000265.hg.1 & 1.547355 & 0.001402 & Up \\
\hline TC04001202.hg.1 & -1.265554 & 0.001405 & Down \\
\hline TC02001892.hg.1 & -1.479308 & 0.00141 & Down \\
\hline TCOX001653.hg.1 & -1.20304 & 0.001413 & Down \\
\hline TC17001939.hg.1 & -1.305775 & 0.001415 & Down \\
\hline TC14001708.hg.1 & 1.42332 & 0.001423 & Up \\
\hline TC20001199.hg.1 & -1.794386 & 0.001426 & Down \\
\hline TC06004094.hg.1 & -1.213016 & 0.001434 & Down \\
\hline TC11003053.hg.1 & 1.483375 & 0.001436 & Up \\
\hline TC01001470.hg.1 & -1.244999 & 0.001439 & Down \\
\hline TC04001675.hg.1 & 1.367312 & 0.001441 & Up \\
\hline TC05001384.hg.1 & -1.20032 & 0.001444 & Down \\
\hline TC22000509.hg.1 & 1.20276 & 0.001449 & Up \\
\hline TC10000687.hg.1 & 1.301899 & 0.001454 & Up \\
\hline TC1 1000849.hg.1 & 1.465989 & 0.001457 & Up \\
\hline TC15002506.hg.1 & 1.432903 & 0.00146 & Up \\
\hline TC14001281.hg.1 & -1.515784 & 0.001462 & Down \\
\hline TC05002143.hg.1 & -1.507259 & 0.001465 & Down \\
\hline TC07001428.hg.1 & -1.281883 & 0.001467 & Down \\
\hline TC07002487.hg.1 & -1.220035 & 0.001472 & Down \\
\hline TC13000434.hg.1 & -1.269599 & 0.001475 & Down \\
\hline TC17000700.hg.1 & 1.521397 & 0.001478 & Up \\
\hline TC06000356.hg.1 & 1.234269 & 0.00148 & Up \\
\hline TC07000879.hg.1 & 1.442272 & 0.001483 & Up \\
\hline TC16000168.hg.1 & -1.307892 & 0.001485 & Down \\
\hline TC07002026.hg.1 & -1.230563 & 0.001491 & Down \\
\hline TC15002522.hg.1 & -1.247948 & 0.001493 & Down \\
\hline TC03002349.hg.1 & 1.308143 & 0.001496 & Up \\
\hline TC04001294.hg.1 & -1.255584 & 0.001498 & Down \\
\hline TC01005264.hg.1 & -1.438176 & 0.001501 & Down \\
\hline TC05002959.hg.1 & -1.301601 & 0.001504 & Down \\
\hline TC08001642.hg.1 & 1.379421 & 0.001506 & Up \\
\hline TC12001758.hg.1 & -1.281904 & 0.001509 & Down \\
\hline TC1 1002955.hg.1 & 1.23146 & 0.001514 & Up \\
\hline TC01006001.hg.1 & -1.231238 & 0.001519 & Down \\
\hline TC11001275.hg.1 & -1.326731 & 0.001522 & Down \\
\hline TC09000835.hg.1 & -1.232379 & 0.001524 & Down \\
\hline TC10001178.hg.1 & 1.259514 & 0.001527 & Up \\
\hline TC01004281.hg.1 & -1.281757 & 0.00153 & Down \\
\hline TC12002368.hg.1 & -1.214252 & 0.001532 & Down \\
\hline TC03002510.hg.1 & -1.22681 & 0.001537 & Down \\
\hline
\end{tabular}

Table 2 (continued)

\begin{tabular}{|c|c|c|c|}
\hline Probe set ID & Fold change & $p$-value & Gene feature \\
\hline TC18000199.hg.1 & 1.359443 & 0.00154 & Up \\
\hline TC03000889.hg.1 & -1.378763 & 0.001548 & Down \\
\hline TC16001163.hg.1 & 1.522691 & 0.001553 & Up \\
\hline TC16000573.hg.1 & -1.384428 & 0.001561 & Down \\
\hline TC22001327.hg.1 & -1.261884 & 0.001563 & Down \\
\hline TC19000423.hg.1 & -1.349595 & 0.001566 & Down \\
\hline TC19000016.hg.1 & -1.203688 & 0.001569 & Down \\
\hline TC12000395.hg.1 & 1.483422 & 0.001571 & Up \\
\hline TC07002238.hg.1 & 1.258405 & 0.001574 & Up \\
\hline TC0X000584.hg.1 & 1.568076 & 0.001576 & Up \\
\hline TC15002609.hg.1 & 1.230717 & 0.001584 & Up \\
\hline TC05002180.hg.1 & -1.289242 & 0.001587 & Down \\
\hline TC12001542.hg.1 & 1.248048 & 0.001594 & Up \\
\hline TC09002127.hg.1 & -1.251934 & 0.001597 & Down \\
\hline TC01005541.hg.1 & 1.349643 & 0.0016 & Up \\
\hline TC13000759.hg.1 & 1.325838 & 0.001602 & Up \\
\hline TC03002654.hg.1 & 1.573194 & 0.001605 & Up \\
\hline TC04000264.hg.1 & -1.242144 & 0.001607 & Down \\
\hline TC10001183.hg.1 & 1.254169 & 0.00161 & Up \\
\hline TC02001767.hg.1 & 1.501282 & 0.001613 & Up \\
\hline TC16001787.hg.1 & -1.23458 & 0.001615 & Down \\
\hline TC16001996.hg.1 & -1.413337 & 0.00162 & Down \\
\hline TC16001381.hg.1 & -1.250738 & 0.001626 & Down \\
\hline TC20001170.hg.1 & -1.215293 & 0.001628 & Down \\
\hline TC0X001367.hg.1 & 1.327188 & 0.001631 & Up \\
\hline TC05002924.hg.1 & -1.38887 & 0.001639 & Down \\
\hline TC01005172.hg.1 & -1.413469 & 0.001646 & Down \\
\hline TC06000650.hg.1 & -1.207228 & 0.001649 & Down \\
\hline TC02004514.hg.1 & -1.251876 & 0.001652 & Down \\
\hline TC14000850.hg.1 & -1.358753 & 0.001654 & Down \\
\hline TC04001198.hg.1 & 1.27254 & 0.001657 & Up \\
\hline TC0X000282.hg.1 & -1.384996 & 0.001659 & Down \\
\hline TC08000176.hg.1 & -1.262382 & 0.001662 & Down \\
\hline TC19002431.hg.1 & -1.271144 & 0.001667 & Down \\
\hline TC07000154.hg.1 & 1.214019 & 0.001672 & Up \\
\hline TC15001223.hg.1 & 1.41761 & 0.001677 & Up \\
\hline TC15000346.hg.1 & 1.607314 & 0.001685 & Up \\
\hline TC02003220.hg.1 & -1.220085 & 0.001693 & Down \\
\hline TC02001567.hg.1 & -1.514096 & 0.001696 & Down \\
\hline TC07003062.hg.1 & 1.473937 & 0.001701 & Up \\
\hline TC16001481.hg.1 & -1.256589 & 0.001703 & Down \\
\hline TC11002658.hg.1 & -1.243073 & 0.001706 & Down \\
\hline TC20000800.hg.1 & -1.22335 & 0.001714 & Down \\
\hline TC01004190.hg.1 & -1.280267 & 0.001719 & Down \\
\hline TC10000755.hg.1 & -1.500832 & 0.001727 & Down \\
\hline TC07002475.hg.1 & 1.335462 & 0.001748 & Up \\
\hline TC09000471.hg.1 & -1.258358 & 0.001758 & Down \\
\hline TC0X001618.hg.1 & -1.273233 & 0.001761 & Down \\
\hline TC01003152.hg.1 & 1.441204 & 0.001768 & Up \\
\hline TC20000841.hg.1 & -1.313791 & 0.001771 & Down \\
\hline TC05002975.hg.1 & -1.309682 & 0.001779 & Down \\
\hline TC08001847.hg.1 & 1.406037 & 0.001784 & Up \\
\hline TC02003913.hg.1 & -1.372422 & 0.001786 & Down \\
\hline
\end{tabular}


Table 2 (continued)

\begin{tabular}{|c|c|c|c|}
\hline Probe set ID & Fold change & $p$-value & Gene feature \\
\hline TC10002891.hg.1 & -1.201915 & 0.001792 & Down \\
\hline TC19001852.hg.1 & 1.491505 & 0.001794 & Up \\
\hline TC03002962.hg.1 & -1.248919 & 0.001805 & Down \\
\hline TC01005988.hg.1 & -1.415789 & 0.001812 & Down \\
\hline TC01006043.hg.1 & -1.359798 & 0.001818 & Down \\
\hline TC14000381.hg.1 & 1.281079 & 0.00182 & Up \\
\hline TC12000580.hg.1 & 1.735542 & 0.001825 & Up \\
\hline TC01002098.hg.1 & -1.253355 & 0.001831 & Down \\
\hline TC02001032.hg.1 & -1.303562 & 0.001833 & Down \\
\hline TC04001271.hg.1 & 1.318074 & 0.001836 & Up \\
\hline TC11000769.hg.1 & -1.271684 & 0.001838 & Down \\
\hline TC03001559.hg.1 & 1.416685 & 0.001844 & Up \\
\hline TC09001810.hg.1 & -1.270957 & 0.001846 & Down \\
\hline TC08001514.hg.1 & -1.204519 & 0.001851 & Down \\
\hline TC14001530.hg.1 & -1.323404 & 0.001854 & Down \\
\hline TC02003388.hg.1 & -1.23358 & 0.001864 & Down \\
\hline TC0X000822.hg.1 & -1.718844 & 0.001867 & Down \\
\hline TC17002619.hg.1 & -1.211196 & 0.001877 & Down \\
\hline TC07000080.hg.1 & -1.269449 & 0.00188 & Down \\
\hline TC09002217.hg.1 & -1.319933 & 0.001882 & Down \\
\hline TC01003082.hg.1 & 1.682659 & 0.001894 & Up \\
\hline TC01003146.hg.1 & 1.682659 & 0.001894 & Up \\
\hline TC07001692.hg.1 & 1.305275 & 0.001901 & Up \\
\hline TC04001091.hg.1 & -1.284332 & 0.001903 & Down \\
\hline TC15001480.hg.1 & -1.38294 & 0.001911 & Down \\
\hline TC06002096.hg.1 & -1.296343 & 0.001914 & Down \\
\hline TC20001131.hg.1 & 1.239192 & 0.001916 & Up \\
\hline TC10001843.hg.1 & -1.279966 & 0.001919 & Down \\
\hline TC06002013.hg.1 & 1.468244 & 0.001924 & Up \\
\hline TC04001248.hg.1 & 1.498609 & 0.001927 & Up \\
\hline TC1 1002681.hg.1 & 1.284111 & 0.001929 & Up \\
\hline TC07003058.hg.1 & -1.28452 & 0.00194 & Down \\
\hline TC19002000.hg.1 & -1.245035 & 0.001942 & Down \\
\hline TC22000340.hg.1 & 1.589672 & 0.001945 & Up \\
\hline TC01002805.hg.1 & 1.202882 & 0.00195 & Up \\
\hline TC04000366.hg.1 & 1.409609 & 0.001953 & Up \\
\hline TC09000159.hg.1 & -1.312322 & 0.001958 & Down \\
\hline TC10001198.hg.1 & -1.357252 & 0.00196 & Down \\
\hline TC06000940.hg.1 & 1.265177 & 0.001963 & Up \\
\hline TC17001752.hg.1 & 1.334898 & 0.001965 & Up \\
\hline TC22001201.hg.1 & -2.439349 & 0.001973 & Down \\
\hline TC05003153.hg.1 & -1.240737 & 0.001976 & Down \\
\hline TC17002053.hg.1 & -1.244021 & 0.001978 & Down \\
\hline TC01003999.hg.1 & -1.700442 & 0.001986 & Down \\
\hline TC04002855.hg.1 & -1.268654 & 0.001989 & Down \\
\hline TC14000667.hg.1 & 1.399213 & 0.001991 & Up \\
\hline TC04001141.hg.1 & -1.525018 & 0.001994 & Down \\
\hline TC01005544.hg.1 & 1.237713 & 0.002002 & Up \\
\hline TC0X001406.hg.1 & 1.230021 & 0.002007 & Up \\
\hline TC05001662.hg.1 & 1.440258 & 0.00201 & Up \\
\hline TC1 1000282.hg.1 & -1.356168 & 0.002015 & Down \\
\hline TC21000954.hg.1 & -1.296443 & 0.002017 & Down \\
\hline TC08001129.hg.1 & -1.472614 & 0.002023 & Down \\
\hline
\end{tabular}

Table 2 (continued)

\begin{tabular}{|c|c|c|c|}
\hline Probe set ID & Fold change & $p$-value & Gene feature \\
\hline TC06004012.hg.1 & -1.212754 & 0.002028 & Down \\
\hline TC17002825.hg.1 & 1.349775 & 0.00203 & Up \\
\hline TC06004016.hg.1 & -1.239402 & 0.002038 & Down \\
\hline TC10002884.hg.1 & 1.431763 & 0.002043 & Up \\
\hline TC05003136.hg.1 & 1.353844 & 0.002049 & Up \\
\hline TC12001421.hg.1 & 1.333251 & 0.002056 & Up \\
\hline TC15001280.hg.1 & 1.343707 & 0.00206 & Up \\
\hline TC15001283.hg.1 & 1.343707 & 0.00206 & Up \\
\hline TC14000739.hg.1 & 1.38166 & 0.002103 & Up \\
\hline TC10002474.hg.1 & 1.331327 & 0.002111 & Up \\
\hline TC14000601.hg.1 & -1.288544 & 0.002113 & Down \\
\hline TC11002989.hg.1 & -1.321635 & 0.002116 & Down \\
\hline TCOX001753.hg.1 & -1.205232 & 0.002119 & Down \\
\hline TC17000925.hg.1 & 1.272254 & 0.002121 & Up \\
\hline TC17000253.hg.1 & 1.345784 & 0.002124 & Up \\
\hline TC21000698.hg.1 & -1.374031 & 0.002137 & Down \\
\hline TC02001905.hg.1 & 1.328816 & 0.002147 & Up \\
\hline TC06002227.hg.1 & -1.498561 & 0.00215 & Down \\
\hline TC07000555.hg.1 & -1.337119 & 0.002152 & Down \\
\hline TC09002594.hg.1 & 1.339428 & 0.002155 & Up \\
\hline TC12001568.hg.1 & 1.341653 & 0.002157 & Up \\
\hline TC05001229.hg.1 & 1.360326 & 0.002165 & Up \\
\hline TC04001597.hg.1 & -1.331486 & 0.00217 & Down \\
\hline TC07002682.hg.1 & -1.221462 & 0.002173 & Down \\
\hline TC08001671.hg.1 & 1.488276 & 0.002176 & Up \\
\hline TC12002539.hg.1 & -1.275317 & 0.002178 & Down \\
\hline TC09002851.hg.1 & -1.333711 & 0.002181 & Down \\
\hline TC01000483.hg.1 & 1.552037 & 0.002186 & Up \\
\hline TC06003970.hg.1 & -1.368856 & 0.002189 & Down \\
\hline TC15002586.hg.1 & -1.340035 & 0.002191 & Down \\
\hline TC12002612.hg.1 & -1.256137 & 0.002196 & Down \\
\hline TC09001443.hg.1 & -1.207418 & 0.002212 & Down \\
\hline TC03001682.hg.1 & 1.260171 & 0.002215 & Up \\
\hline TC17000782.hg.1 & -1.204102 & 0.002217 & Down \\
\hline TC15000597.hg.1 & 1.213718 & 0.00222 & Up \\
\hline TC06003660.hg.1 & -1.237973 & 0.002222 & Down \\
\hline TC17002039.hg.1 & 1.340985 & 0.002225 & Up \\
\hline TC03002816.hg.1 & -1.247147 & 0.002228 & Down \\
\hline TC09000100.hg.1 & -1.377179 & 0.00223 & Down \\
\hline TC12002319.hg.1 & -1.211631 & 0.002235 & Down \\
\hline TC02002799.hg.1 & -1.264613 & 0.002241 & Down \\
\hline TC07000677.hg.1 & -1.215502 & 0.002246 & Down \\
\hline TC07000516.hg.1 & 1.289644 & 0.002248 & Up \\
\hline TCOX000501.hg.1 & -1.324115 & 0.002254 & Down \\
\hline TC16001448.hg.1 & 1.236904 & 0.002259 & Up \\
\hline TC07002912.hg.1 & -1.326523 & 0.002266 & Down \\
\hline TC10001062.hg.1 & 1.412222 & 0.002269 & Up \\
\hline TC10000761.hg.1 & 1.583303 & 0.002272 & Up \\
\hline TC05000214.hg.1 & -1.353026 & 0.002274 & Down \\
\hline TC09002468.hg.1 & 1.209262 & 0.002282 & Up \\
\hline TC12001658.hg.1 & 1.437781 & 0.002285 & Up \\
\hline TC03001924.hg.1 & 1.271901 & 0.00229 & Up \\
\hline TC03003246.hg.1 & 1.207269 & 0.002295 & Up \\
\hline
\end{tabular}


Table 2 (continued)

\begin{tabular}{|c|c|c|c|}
\hline Probe set ID & Fold change & $p$-value & Gene feature \\
\hline TC14001678.hg.1 & 1.530119 & 0.0023 & Up \\
\hline TC03002629.hg.1 & -1.225872 & 0.002313 & Down \\
\hline TC20001519.hg.1 & -1.220912 & 0.002321 & Down \\
\hline TC01006051.hg.1 & -1.336503 & 0.002324 & Down \\
\hline TC06002665.hg.1 & 1.461845 & 0.002331 & Up \\
\hline TC02001522.hg.1 & -1.248588 & 0.002334 & Down \\
\hline TC12002293.hg.1 & -1.209515 & 0.002342 & Down \\
\hline TC21000628.hg.1 & -1.231307 & 0.002344 & Down \\
\hline TC12001919.hg.1 & -1.351643 & 0.002347 & Down \\
\hline TC02001799.hg.1 & -2.445831 & 0.00235 & Down \\
\hline TC17000742.hg.1 & -1.320632 & 0.002357 & Down \\
\hline TC04002002.hg.1 & -1.303665 & 0.00236 & Down \\
\hline TCOX001976.hg.1 & 1.267861 & 0.00237 & Up \\
\hline TC1 1002465.hg.1 & -1.277875 & 0.002383 & Down \\
\hline TC09001504.hg.1 & -1.312141 & 0.002388 & Down \\
\hline TC05000162.hg.1 & 1.294054 & 0.002391 & Up \\
\hline TC06003710.hg.1 & -1.344179 & 0.002399 & Down \\
\hline TC15002646.hg.1 & 1.2472 & 0.002401 & Up \\
\hline TC03002025.hg.1 & -1.217553 & 0.002417 & Down \\
\hline TC08000782.hg.1 & 1.360634 & 0.002422 & Up \\
\hline TC12001854.hg.1 & -1.235064 & 0.002427 & Down \\
\hline TC15000426.hg.1 & -1.229315 & 0.002435 & Down \\
\hline TC15002177.hg.1 & 1.279118 & 0.00244 & Up \\
\hline TC22001207.hg.1 & -1.203482 & 0.002451 & Down \\
\hline TC03002554.hg.1 & -1.220846 & 0.002458 & Down \\
\hline TC1 1000720.hg.1 & 1.488337 & 0.002461 & Up \\
\hline TC12003053.hg.1 & 1.261293 & 0.002471 & Up \\
\hline TC01003159.hg.1 & 1.455932 & 0.002479 & Up \\
\hline TC03000673.hg.1 & -1.307368 & 0.002484 & Down \\
\hline TC12001455.hg.1 & -1.316009 & 0.002487 & Down \\
\hline TC05000503.hg.1 & -1.223516 & 0.002497 & Down \\
\hline TC01002453.hg.1 & 1.461387 & 0.002508 & Up \\
\hline TC09002781.hg.1 & 1.429014 & 0.002518 & Up \\
\hline TC02000495.hg.1 & 1.291697 & 0.002529 & Up \\
\hline TC20001320.hg.1 & 1.297954 & 0.002531 & Up \\
\hline TC01004677.hg.1 & -1.285666 & 0.002534 & Down \\
\hline TC03000004.hg.1 & -1.234276 & 0.002539 & Down \\
\hline TC07000031.hg.1 & 1.223867 & 0.002557 & Up \\
\hline TC18000275.hg.1 & -1.277381 & 0.00256 & Down \\
\hline TC01003175.hg.1 & 1.43838 & 0.002565 & Up \\
\hline TC18000216.hg.1 & -1.286168 & 0.002567 & Down \\
\hline TC01006192.hg.1 & 1.268851 & 0.002573 & Up \\
\hline TC08000259.hg.1 & -1.344703 & 0.002583 & Down \\
\hline TC04001562.hg.1 & 1.450228 & 0.002593 & Up \\
\hline TC03003056.hg.1 & 1.215057 & 0.002601 & Up \\
\hline TC12001883.hg.1 & 1.569242 & 0.002609 & Up \\
\hline TC01003899.hg.1 & -1.28947 & 0.002617 & Down \\
\hline TCOY000064.hg.1 & 1.200448 & 0.002628 & Up \\
\hline TCOY000173.hg.1 & 1.200448 & 0.002628 & Up \\
\hline TC09000674.hg.1 & -1.2102 & 0.002635 & Down \\
\hline TC04000005.hg.1 & -1.295829 & 0.002638 & Down \\
\hline TC17002085.hg.1 & -1.281199 & 0.00264 & Down \\
\hline TC01001139.hg.1 & 1.508166 & 0.00265 & Up \\
\hline
\end{tabular}

Table 2 (continued)

\begin{tabular}{|c|c|c|c|}
\hline Probe set ID & Fold change & $p$-value & Gene feature \\
\hline TC20001755.hg.1 & 1.352651 & 0.002656 & Up \\
\hline TC02002970.hg.1 & -1.429192 & 0.002658 & Down \\
\hline TC07002772.hg.1 & 1.373647 & 0.002661 & Up \\
\hline TC11001556.hg.1 & -1.206529 & 0.002666 & Down \\
\hline TC04002294.hg.1 & -1.401452 & 0.002671 & Down \\
\hline TC01005305.hg.1 & 1.356928 & 0.002676 & Up \\
\hline TC15001904.hg.1 & -1.272703 & 0.002682 & Down \\
\hline TC20000317.hg.1 & 1.430596 & 0.002687 & Up \\
\hline TC08000740.hg.1 & 1.402556 & 0.002692 & Up \\
\hline TC09001635.hg.1 & -1.276086 & 0.0027 & Down \\
\hline TC13001064.hg.1 & -1.26174 & 0.002702 & Down \\
\hline TC02003591.hg.1 & 1.299115 & 0.00271 & Up \\
\hline TC07002773.hg.1 & 1.331845 & 0.002721 & Up \\
\hline TC05002891.hg.1 & -1.259569 & 0.002726 & Down \\
\hline TC09002429.hg.1 & -1.205487 & 0.002728 & Down \\
\hline TC06001125.hg.1 & -1.232463 & 0.002734 & Down \\
\hline TC03002933.hg.1 & -1.211047 & 0.002744 & Down \\
\hline TC09002223.hg.1 & -1.202365 & 0.002746 & Down \\
\hline TC01004768.hg.1 & -1.224528 & 0.002757 & Down \\
\hline TC08000722.hg.1 & 1.365601 & 0.002762 & Up \\
\hline TC03001348.hg.1 & -1.239947 & 0.002767 & Down \\
\hline TC09002651.hg.1 & -1.69314 & 0.002778 & Down \\
\hline TC07002574.hg.1 & -1.24146 & 0.002788 & Down \\
\hline TC08000076.hg.1 & 1.404643 & 0.002793 & Up \\
\hline TC05000182.hg.1 & 1.301406 & 0.002804 & Up \\
\hline TCOX000127.hg.1 & -1.20556 & 0.002809 & Down \\
\hline TC03000962.hg.1 & -1.382564 & 0.002819 & Down \\
\hline TC07000246.hg.1 & -1.267632 & 0.002827 & Down \\
\hline TC07000320.hg.1 & 1.341276 & 0.002835 & Up \\
\hline TC1 1002734.hg.1 & 1.297811 & 0.002843 & Up \\
\hline TC17001691.hg.1 & -1.271637 & 0.002848 & Down \\
\hline TC01001837.hg.1 & 1.550832 & 0.00285 & Up \\
\hline TC20000915.hg.1 & -1.232658 & 0.002863 & Down \\
\hline TC02003364.hg.1 & -1.266053 & 0.002871 & Down \\
\hline TCOX001104.hg.1 & 1.359394 & 0.002889 & Up \\
\hline TC01000711.hg.1 & -1.216828 & 0.002892 & Down \\
\hline TC17000989.hg.1 & -1.21244 & 0.002894 & Down \\
\hline TC02002022.hg.1 & 1.263206 & 0.002897 & Up \\
\hline TC07002067.hg.1 & -1.31306 & 0.0029 & Down \\
\hline TC07001289.hg.1 & -1.351691 & 0.002902 & Down \\
\hline TC01000295.hg.1 & 1.305782 & 0.002905 & Up \\
\hline TC06000662.hg.1 & 1.480994 & 0.002907 & Up \\
\hline TC19002432.hg.1 & -1.286462 & 0.00291 & Down \\
\hline TC14002087.hg.1 & -1.270823 & 0.002918 & Down \\
\hline TC05002826.hg.1 & 1.273654 & 0.00292 & Up \\
\hline TC04001876.hg.1 & -1.318727 & 0.002936 & Down \\
\hline TC07002081.hg.1 & -1.319864 & 0.002939 & Down \\
\hline TC20001277.hg.1 & 1.278604 & 0.002946 & Up \\
\hline TC05000501.hg.1 & 1.274401 & 0.002962 & Up \\
\hline TC10000590.hg.1 & 1.370535 & 0.002977 & Up \\
\hline TC15001485.hg.1 & -1.291058 & 0.002993 & Down \\
\hline TC15002487.hg.1 & -1.367271 & 0.003006 & Down \\
\hline TC21000774.hg.1 & -1.289989 & 0.003009 & Down \\
\hline
\end{tabular}


Table 2 (continued)

\begin{tabular}{|c|c|c|c|}
\hline Probe set ID & Fold change & $p$-value & Gene feature \\
\hline TC02002679.hg.1 & -1.293013 & 0.003011 & Down \\
\hline TC07001604.hg.1 & 1.354263 & 0.003014 & Up \\
\hline TC12001252.hg.1 & 1.447457 & 0.003016 & Up \\
\hline TC13001458.hg.1 & -1.207147 & 0.003019 & Down \\
\hline TC09002644.hg.1 & -1.26481 & 0.003022 & Down \\
\hline TCOX001878.hg.1 & -1.209099 & 0.003024 & Down \\
\hline TCOX001302.hg.1 & 1.569258 & 0.003027 & Up \\
\hline TC18000294.hg.1 & 1.260461 & 0.003045 & Up \\
\hline TC08000507.hg.1 & -1.315641 & 0.003055 & Down \\
\hline TC08000970.hg.1 & -1.224392 & 0.003058 & Down \\
\hline TC05002688.hg.1 & -1.2392 & 0.00306 & Down \\
\hline TC1 1003229.hg.1 & -1.251246 & 0.003063 & Down \\
\hline TC09002325.hg.1 & -1.270503 & 0.003076 & Down \\
\hline TC10002238.hg.1 & -1.329862 & 0.003081 & Down \\
\hline TC06001064.hg.1 & -1.274065 & 0.003092 & Down \\
\hline TC02003391.hg.1 & -1.362446 & 0.003099 & Down \\
\hline TC10000133.hg.1 & -1.238252 & 0.003103 & Down \\
\hline TC10000136.hg.1 & -1.238252 & 0.003103 & Down \\
\hline TC03002201.hg.1 & -1.331047 & 0.003112 & Down \\
\hline TC07001869.hg.1 & -1.488884 & 0.003118 & Down \\
\hline TC15001493.hg.1 & -1.20754 & 0.003125 & Down \\
\hline TC15002311.hg.1 & -1.221615 & 0.003136 & Down \\
\hline TC07001788.hg.1 & -1.398937 & 0.003143 & Down \\
\hline TC06003354.hg.1 & -1.228478 & 0.003146 & Down \\
\hline TC07002240.hg.1 & 1.460799 & 0.003151 & Up \\
\hline TC01000348.hg.1 & -1.280176 & 0.003162 & Down \\
\hline TC05000429.hg.1 & -1.204846 & 0.003172 & Down \\
\hline TC10002375.hg.1 & -1.214255 & 0.003175 & Down \\
\hline TC1 1001740.hg.1 & 1.307581 & 0.003177 & Up \\
\hline TC11000061.hg.1 & 1.501956 & 0.00318 & Up \\
\hline TC16000236.hg.1 & 1.306824 & 0.003203 & Up \\
\hline TC07001599.hg.1 & -1.353408 & 0.003211 & Down \\
\hline TC08001580.hg.1 & -1.29275 & 0.003219 & Down \\
\hline TC20000677.hg.1 & -1.209774 & 0.003221 & Down \\
\hline TCOX001145.hg.1 & 1.32182 & 0.003227 & Up \\
\hline TC19000162.hg.1 & -1.296602 & 0.003232 & Down \\
\hline TCOX001119.hg.1 & 1.23651 & 0.003258 & Up \\
\hline TC16001238.hg.1 & 1.548063 & 0.00326 & Up \\
\hline TC04000904.hg.1 & -1.325403 & 0.003281 & Down \\
\hline TC01004933.hg.1 & 1.296042 & 0.003284 & Up \\
\hline TC03002799.hg.1 & 1.233829 & 0.003286 & Up \\
\hline TC17001438.hg.1 & -1.282082 & 0.003307 & Down \\
\hline TC12002384.hg.1 & 1.229617 & 0.003315 & Up \\
\hline TC06003692.hg.1 & -1.256837 & 0.003323 & Down \\
\hline TC06001254.hg.1 & -1.229231 & 0.003328 & Down \\
\hline TC21000398.hg.1 & -1.279981 & 0.003333 & Down \\
\hline TC06000858.hg.1 & 1.611132 & 0.003341 & Up \\
\hline TC15001164.hg.1 & -1.235207 & 0.003343 & Down \\
\hline TC01000551.hg.1 & 1.249583 & 0.003348 & Up \\
\hline TC12003054.hg.1 & 1.220865 & 0.003354 & Up \\
\hline TC18000207.hg.1 & 1.207556 & 0.003359 & Up \\
\hline TC16001555.hg.1 & -1.235816 & 0.003369 & Down \\
\hline
\end{tabular}

Table 2 (continued)

\begin{tabular}{|c|c|c|c|}
\hline Probe set ID & Fold change & p-value & Gene feature \\
\hline TC07000470.hg.1 & 1.268777 & 0.003372 & Up \\
\hline TC06001808.hg.1 & 1.248183 & 0.003385 & Up \\
\hline TC01000364.hg.1 & -1.330246 & 0.003387 & Down \\
\hline TC12000919.hg.1 & 1.563172 & 0.00339 & Up \\
\hline TC05001064.hg.1 & 1.565417 & 0.003393 & Up \\
\hline TC17002281.hg.1 & -1.200596 & 0.003411 & Down \\
\hline TC10000082.hg.1 & -1.375037 & 0.00346 & Down \\
\hline TC02003240.hg.1 & -1.307516 & 0.003481 & Down \\
\hline TC04001241.hg.1 & -1.290744 & 0.003491 & Down \\
\hline TC02004324.hg.1 & -1.232763 & 0.003494 & Down \\
\hline TC10002413.hg.1 & -1.245515 & 0.003515 & Down \\
\hline TC16001946.hg.1 & -1.22412 & 0.003525 & Down \\
\hline TC02002923.hg.1 & -1.276668 & 0.003528 & Down \\
\hline TC09002766.hg.1 & -1.258491 & 0.003535 & Down \\
\hline TC09000642.hg.1 & -1.347036 & 0.003538 & Down \\
\hline TC02001155.hg.1 & -1.210161 & 0.003548 & Down \\
\hline TC17000690.hg.1 & -1.278329 & 0.003556 & Down \\
\hline TC22000690.hg.1 & -1.201581 & 0.003564 & Down \\
\hline TC03001758.hg.1 & -1.260934 & 0.003566 & Down \\
\hline TC10002858.hg.1 & -1.261472 & 0.0036 & Down \\
\hline TC01001573.hg.1 & -1.31615 & 0.003608 & Down \\
\hline TC12000943.hg.1 & -1.20209 & 0.003613 & Down \\
\hline TC07000478.hg.1 & -1.269749 & 0.003626 & Down \\
\hline TC22001006.hg.1 & -1.20719 & 0.003636 & Down \\
\hline TC21000941.hg.1 & -1.364074 & 0.003655 & Down \\
\hline TC07002774.hg.1 & -1.224753 & 0.00366 & Down \\
\hline TC06003965.hg.1 & -1.238846 & 0.003665 & Down \\
\hline TCOY000049.hg.1 & -1.363073 & 0.003675 & Down \\
\hline TC04000236.hg.1 & -1.212799 & 0.003681 & Down \\
\hline TCOX001770.hg.1 & -1.33727 & 0.003691 & Down \\
\hline TC06003404.hg.1 & -1.327154 & 0.003694 & Down \\
\hline TC05001562.hg.1 & -1.380095 & 0.003699 & Down \\
\hline TC05001222.hg.1 & -1.267421 & 0.003725 & Down \\
\hline TC01005060.hg.1 & -1.314409 & 0.003727 & Down \\
\hline TC03000359.hg.1 & -1.202882 & 0.003751 & Down \\
\hline TC08001194.hg.1 & -1.424464 & 0.003753 & Down \\
\hline TC09001332.hg.1 & -1.371182 & 0.003782 & Down \\
\hline TC07001668.hg.1 & -1.221176 & 0.003816 & Down \\
\hline TC06000609.hg.1 & -1.2365 & 0.003834 & Down \\
\hline TC14002171.hg.1 & -1.305933 & 0.003839 & Down \\
\hline TC05003054.hg.1 & -1.216461 & 0.003841 & Down \\
\hline TC12000990.hg.1 & -1.45711 & 0.003854 & Down \\
\hline TC18000510.hg.1 & -1.260881 & 0.003862 & Down \\
\hline TC04000456.hg.1 & -1.263786 & 0.00388 & Down \\
\hline TC01000871.hg.1 & -1.21274 & 0.003896 & Down \\
\hline TC16000865.hg.1 & -1.285648 & 0.003899 & Down \\
\hline TC06002606.hg.1 & -1.49097 & 0.003927 & Down \\
\hline TC22000272.hg.1 & -1.554161 & 0.003932 & Down \\
\hline TC06002746.hg.1 & -1.202909 & 0.00394 & Down \\
\hline TCOY000257.hg.1 & -1.281043 & 0.003945 & Down \\
\hline TC18000540.hg.1 & -1.251836 & 0.003948 & Down \\
\hline TC08002048.hg.1 & -1.207051 & 0.003966 & Down \\
\hline
\end{tabular}


Table 2 (continued)

\begin{tabular}{|c|c|c|c|}
\hline Probe set ID & Fold change & p-value & Gene feature \\
\hline TC17002700.hg.1 & -1.235688 & 0.003974 & Down \\
\hline TC05001722.hg.1 & -1.273013 & 0.003982 & Down \\
\hline TC01005183.hg.1 & -1.227973 & 0.003987 & Down \\
\hline TC12002485.hg.1 & -1.260362 & 0.004 & Down \\
\hline TC10001548.hg.1 & -1.301554 & 0.004002 & Down \\
\hline TC13001206.hg.1 & -1.467406 & 0.004005 & Down \\
\hline TC11003065.hg.1 & -1.232606 & 0.004013 & Down \\
\hline TC10000091.hg.1 & -1.407866 & 0.004018 & Down \\
\hline TC06004002.hg.1 & -1.324321 & 0.00402 & Down \\
\hline TC02004420.hg.1 & -1.245413 & 0.004057 & Down \\
\hline TC02002409.hg.1 & -1.296558 & 0.004059 & Down \\
\hline TC05002084.hg.1 & -1.429991 & 0.004067 & Down \\
\hline TC03000687.hg.1 & -1.368897 & 0.004096 & Down \\
\hline TC05002736.hg.1 & -1.269686 & 0.004101 & Down \\
\hline TC11000172.hg.1 & -1.381513 & 0.004117 & Down \\
\hline TC04000860.hg.1 & -1.20631 & 0.004197 & Down \\
\hline TC13001692.hg.1 & -1.204469 & 0.004202 & Down \\
\hline TC03002000.hg.1 & -1.245183 & 0.00421 & Down \\
\hline TC0X000016.hg.1 & -1.216418 & 0.00422 & Down \\
\hline TC01005274.hg.1 & -1.212808 & 0.004223 & Down \\
\hline TC17001702.hg.1 & -1.257121 & 0.004264 & Down \\
\hline TC07002787.hg.1 & -1.2704 & 0.004275 & Down \\
\hline TC11001982.hg.1 & -1.215692 & 0.004283 & Down \\
\hline TC11002457.hg.1 & -1.224026 & 0.004285 & Down \\
\hline TC21000490.hg.1 & -1.222362 & 0.004298 & Down \\
\hline TC02001213.hg.1 & -1.275065 & 0.004309 & Down \\
\hline TC05002802.hg.1 & -1.454294 & 0.004316 & Down \\
\hline TC02002613.hg.1 & -1.202558 & 0.004342 & Down \\
\hline TC07002265.hg.1 & -1.303624 & 0.00435 & Down \\
\hline TC05002711.hg.1 & -1.278714 & 0.00436 & Down \\
\hline TC09000458.hg.1 & -1.295586 & 0.004368 & Down \\
\hline TC15001598.hg.1 & -1.273945 & 0.004373 & Down \\
\hline TC08000107.hg.1 & -1.284986 & 0.004389 & Down \\
\hline TC12002849.hg.1 & -1.258618 & 0.004394 & Down \\
\hline TC06002956.hg.1 & -1.208955 & 0.004397 & Down \\
\hline TC10000426.hg.1 & -1.257512 & 0.004417 & Down \\
\hline TC07000389.hg.1 & -1.257421 & 0.004438 & Down \\
\hline TC16001097.hg.1 & -1.300925 & 0.004443 & Down \\
\hline TC07002791.hg.1 & -1.206028 & 0.004459 & Down \\
\hline TC19001288.hg.1 & -1.237947 & 0.004464 & Down \\
\hline TC16000146.hg.1 & -1.233673 & 0.004467 & Down \\
\hline TC01002560.hg.1 & -1.207402 & 0.004482 & Down \\
\hline TC10002728.hg.1 & -1.272287 & 0.004506 & Down \\
\hline TC12000184.hg.1 & -1.207964 & 0.004508 & Down \\
\hline TC10001956.hg.1 & -1.201634 & 0.004513 & Down \\
\hline TC10001530.hg.1 & -1.212456 & 0.00456 & Down \\
\hline TC17002899.hg.1 & -1.208538 & 0.004573 & Down \\
\hline TC0X000632.hg.1 & -1.3172 & 0.004581 & Down \\
\hline TC17001547.hg.1 & -1.20639 & 0.004589 & Down \\
\hline TC04000311.hg.1 & -1.204792 & 0.004599 & Down \\
\hline TC20001506.hg.1 & -1.26979 & 0.004609 & Down \\
\hline TC06004015.hg.1 & -1.26321 & 0.004612 & Down \\
\hline
\end{tabular}

Table 2 (continued)

\begin{tabular}{|c|c|c|c|}
\hline Probe set ID & Fold change & $p$-value & Gene feature \\
\hline TC01004440.hg.1 & -1.222055 & 0.004622 & Down \\
\hline TC01002542.hg.1 & -1.318907 & 0.004628 & Down \\
\hline TC18000718.hg.1 & -1.231172 & 0.004638 & Down \\
\hline TC04002390.hg.1 & -1.292294 & 0.004716 & Down \\
\hline TC04002908.hg.1 & -1.22141 & 0.004724 & Down \\
\hline TC01001491.hg.1 & -1.255189 & 0.004731 & Down \\
\hline TC19000841.hg.1 & -1.300943 & 0.004755 & Down \\
\hline TC01002756.hg.1 & -1.426354 & 0.00476 & Down \\
\hline TC20000309.hg.1 & -1.304516 & 0.004765 & Down \\
\hline TC1 1002990.hg.1 & -1.235653 & 0.00477 & Down \\
\hline TC03001403.hg.1 & -1.314851 & 0.004799 & Down \\
\hline TC06003498.hg.1 & -1.355157 & 0.004802 & Down \\
\hline TC09002774.hg.1 & -1.224146 & 0.00482 & Down \\
\hline TC10000250.hg.1 & -1.330922 & 0.004846 & Down \\
\hline TC16000333.hg.1 & -1.201118 & 0.004848 & Down \\
\hline TC22001283.hg.1 & -1.280786 & 0.004859 & Down \\
\hline TC10001915.hg.1 & -1.228546 & 0.004864 & Down \\
\hline TC11000196.hg.1 & -1.265687 & 0.004869 & Down \\
\hline TC02004125.hg.1 & -1.231632 & 0.004898 & Down \\
\hline TC15002307.hg.1 & -1.255684 & 0.0049 & Down \\
\hline TC03003205.hg.1 & -1.249546 & 0.004913 & Down \\
\hline TC01002776.hg.1 & -1.316093 & 0.004939 & Down \\
\hline TC03001745.hg.1 & -1.299476 & 0.004944 & Down \\
\hline TC06003126.hg.1 & -1.286256 & 0.004952 & Down \\
\hline TC01002773.hg.1 & -1.218511 & 0.004965 & Down \\
\hline TC02002927.hg.1 & -1.311688 & 0.004983 & Down \\
\hline TC01004326.hg.1 & -1.204881 & 0.004986 & Down \\
\hline TC21000729.hg.1 & -1.200973 & 0.004988 & Down \\
\hline TC22000110.hg.1 & -1.205007 & 0.004999 & Down \\
\hline TC09001132.hg.1 & -1.271309 & 0.005012 & Down \\
\hline TC10000232.hg.1 & -1.248461 & 0.005017 & Down \\
\hline TC05003302.hg.1 & -1.255306 & 0.005019 & Down \\
\hline TC20001224.hg.1 & -1.32063 & 0.005025 & Down \\
\hline TC01005383.hg.1 & -1.256589 & 0.005027 & Down \\
\hline TCOX001718.hg.1 & -1.226406 & 0.00504 & Down \\
\hline TC14000707.hg.1 & -1.204295 & 0.005058 & Down \\
\hline TC14001715.hg.1 & -1.346526 & 0.005061 & Down \\
\hline TC07002764.hg.1 & -1.200408 & 0.005079 & Down \\
\hline TC06003509.hg.1 & -1.230654 & 0.005084 & Down \\
\hline TC05002436.hg.1 & -1.273183 & 0.005118 & Down \\
\hline TC05000078.hg.1 & -1.23626 & 0.005121 & Down \\
\hline TC01006128.hg.1 & -1.328935 & 0.005126 & Down \\
\hline TC06003211.hg.1 & -1.277803 & 0.005157 & Down \\
\hline TC10000904.hg.1 & -1.247917 & 0.00516 & Down \\
\hline TC01001668.hg.1 & -1.212246 & 0.005206 & Down \\
\hline TC21000493.hg.1 & -1.236162 & 0.005232 & Down \\
\hline TC09000456.hg.1 & -1.266448 & 0.00524 & Down \\
\hline TC09002485.hg.1 & -1.263839 & 0.005256 & Down \\
\hline TC20001556.hg.1 & -1.279838 & 0.005284 & Down \\
\hline TC02002768.hg.1 & -1.253353 & 0.005287 & Down \\
\hline TC10002113.hg.1 & -1.217268 & 0.005328 & Down \\
\hline TC12001434.hg.1 & -1.297937 & 0.005333 & Down \\
\hline
\end{tabular}


Table 2 (continued)

\begin{tabular}{|c|c|c|c|}
\hline Probe set ID & Fold change & p-value & Gene feature \\
\hline TC05001581.hg.1 & -1.260177 & 0.005344 & Down \\
\hline TC15002099.hg.1 & -1.266836 & 0.005352 & Down \\
\hline TC10001912.hg.1 & -1.349501 & 0.005391 & Down \\
\hline TC11002900.hg.1 & -1.284679 & 0.005393 & Down \\
\hline TC01005460.hg.1 & -1.207368 & 0.005401 & Down \\
\hline TC02001846.hg.1 & -1.402528 & 0.005406 & Down \\
\hline TC09000907.hg.1 & -1.24214 & 0.005409 & Down \\
\hline TC0X001572.hg.1 & -1.33578 & 0.005411 & Down \\
\hline TC09001363.hg.1 & -1.240359 & 0.005414 & Down \\
\hline TC10001686.hg.1 & -1.295313 & 0.005458 & Down \\
\hline TC08000285.hg.1 & -1.288083 & 0.005468 & Down \\
\hline TC07000767.hg.1 & -1.290819 & 0.005474 & Down \\
\hline TC13000606.hg.1 & -1.330779 & 0.005479 & Down \\
\hline TC04001792.hg.1 & -1.242708 & 0.005481 & Down \\
\hline TC02003857.hg.1 & -1.268582 & 0.005492 & Down \\
\hline TC01003317.hg.1 & -1.440286 & 0.005531 & Down \\
\hline TC12000588.hg.1 & -1.213828 & 0.005541 & Down \\
\hline TC20001554.hg.1 & -1.207139 & 0.005544 & Down \\
\hline TC07001657.hg.1 & -1.419211 & 0.005577 & Down \\
\hline TC09002148.hg.1 & -1.279893 & 0.00559 & Down \\
\hline TC15001221.hg.1 & -1.210747 & 0.005658 & Down \\
\hline TC11001828.hg.1 & -1.200582 & 0.00566 & Down \\
\hline TC13000062.hg.1 & -1.283458 & 0.005697 & Down \\
\hline TC05001353.hg.1 & -1.216892 & 0.005699 & Down \\
\hline TC01004219.hg.1 & -1.205464 & 0.005702 & Down \\
\hline TC01005835.hg.1 & -1.269379 & 0.005725 & Down \\
\hline TC06000930.hg.1 & -1.262508 & 0.00573 & Down \\
\hline TC03002911.hg.1 & -1.233783 & 0.005749 & Down \\
\hline TC02000415.hg.1 & -1.219812 & 0.005759 & Down \\
\hline TC11003125.hg.1 & -1.221046 & 0.005764 & Down \\
\hline TC01000579.hg.1 & -1.203049 & 0.005803 & Down \\
\hline TC14001295.hg.1 & -1.214363 & 0.005808 & Down \\
\hline TC05002238.hg.1 & -1.208419 & 0.005837 & Down \\
\hline TC04000662.hg.1 & -1.270677 & 0.005852 & Down \\
\hline TC02002347.hg.1 & -1.22743 & 0.005858 & Down \\
\hline TC17000164.hg.1 & -1.280195 & 0.005871 & Down \\
\hline TC08001315.hg.1 & -1.408884 & 0.005894 & Down \\
\hline TC03000982.hg.1 & -1.239878 & 0.005896 & Down \\
\hline TC1 1002959.hg.1 & -1.229904 & 0.005933 & Down \\
\hline TC14001177.hg.1 & -1.267289 & 0.005938 & Down \\
\hline TC11003265.hg.1 & -1.234478 & 0.005943 & Down \\
\hline TC01005831.hg.1 & -1.322234 & 0.005946 & Down \\
\hline TC09001793.hg.1 & -1.214475 & 0.005951 & Down \\
\hline TC01003533.hg.1 & -1.45726 & 0.00598 & Down \\
\hline TC06002968.hg.1 & -1.357034 & 0.00599 & Down \\
\hline TC0X000285.hg.1 & -1.203534 & 0.006068 & Down \\
\hline TC03003239.hg.1 & -1.234383 & 0.006086 & Down \\
\hline TC01004831.hg.1 & -1.238529 & 0.006107 & Down \\
\hline TC22001104.hg.1 & -1.213074 & 0.00614 & Down \\
\hline TC20001570.hg.1 & -1.20816 & 0.006172 & Down \\
\hline TC05000703.hg.1 & -1.311244 & 0.006174 & Down \\
\hline TC18000785.hg.1 & -1.224874 & 0.006179 & Down \\
\hline TC09002372.hg.1 & -1.257884 & 0.006182 & Down \\
\hline
\end{tabular}

Table 2 (continued)

\begin{tabular}{|c|c|c|c|}
\hline Probe set ID & Fold change & p-value & Gene feature \\
\hline TC04000837.hg.1 & -1.230161 & 0.006195 & Down \\
\hline TC11000065.hg.1 & -1.217834 & 0.006275 & Down \\
\hline TC21000071.hg.1 & -1.206307 & 0.00628 & Down \\
\hline TC13001054.hg.1 & -1.273984 & 0.006291 & Down \\
\hline TC02003343.hg.1 & -1.214008 & 0.00634 & Down \\
\hline TC12002692.hg.1 & -1.289651 & 0.006353 & Down \\
\hline TC01005539.hg.1 & -1.21791 & 0.006371 & Down \\
\hline TC07000299.hg.1 & -1.266678 & 0.006379 & Down \\
\hline TC12000625.hg.1 & -1.265515 & 0.006384 & Down \\
\hline TC09002445.hg.1 & -1.334389 & 0.006413 & Down \\
\hline TC05001346.hg.1 & -1.231796 & 0.006493 & Down \\
\hline TC12001821.hg.1 & -1.242271 & 0.006511 & Down \\
\hline TC08000954.hg.1 & -1.26309 & 0.00655 & Down \\
\hline TC12000683.hg.1 & -1.311043 & 0.006566 & Down \\
\hline TC17002263.hg.1 & -1.2066 & 0.006574 & Down \\
\hline TC01001760.hg.1 & -1.277558 & 0.006592 & Down \\
\hline TC17000557.hg.1 & -1.322598 & 0.006597 & Down \\
\hline TC20001196.hg.1 & -1.31133 & 0.0066 & Down \\
\hline TC04001967.hg.1 & -1.290136 & 0.006618 & Down \\
\hline TC14000795.hg.1 & -1.300583 & 0.006633 & Down \\
\hline TC12000745.hg.1 & -1.336139 & 0.006672 & Down \\
\hline TC17001160.hg.1 & -1.232074 & 0.006688 & Down \\
\hline TC09002037.hg.1 & -1.25914 & 0.006701 & Down \\
\hline TC07000189.hg.1 & -1.284554 & 0.006711 & Down \\
\hline TC10002162.hg.1 & -1.331341 & 0.006729 & Down \\
\hline TC10001758.hg.1 & -1.33558 & 0.006732 & Down \\
\hline $\begin{array}{l}\text { TC6_ssto__ } \\
\text { hap7000179.hg.1 }\end{array}$ & -1.266774 & 0.006776 & Down \\
\hline TC05001609.hg.1 & -1.219982 & 0.006802 & Down \\
\hline TC09000397.hg.1 & -1.208158 & 0.006825 & Down \\
\hline TC01000008.hg.1 & -1.260407 & 0.006838 & Down \\
\hline TC05002179.hg.1 & -1.24681 & 0.006955 & Down \\
\hline TC06003468.hg.1 & -1.237969 & 0.007002 & Down \\
\hline TC07000766.hg.1 & -1.246781 & 0.007038 & Down \\
\hline TC03002074.hg.1 & -1.286913 & 0.007049 & Down \\
\hline TC06002573.hg.1 & -1.200492 & 0.007087 & Down \\
\hline TC17002315.hg.1 & -1.224284 & 0.007119 & Down \\
\hline TC17002681.hg.1 & -1.215864 & 0.007147 & Down \\
\hline TC08002507.hg.1 & -1.219674 & 0.007209 & Down \\
\hline TC07001642.hg.1 & -1.262919 & 0.007238 & Down \\
\hline TC04002802.hg.1 & -1.281658 & 0.007272 & Down \\
\hline TC07000507.hg.1 & -1.319983 & 0.007285 & Down \\
\hline TC03000233.hg.1 & -1.267284 & 0.0073 & Down \\
\hline TC17000344.hg.1 & -1.230796 & 0.007318 & Down \\
\hline TC10000974.hg.1 & -1.235907 & 0.007324 & Down \\
\hline TC01002173.hg.1 & -1.204552 & 0.007339 & Down \\
\hline TC03000641.hg.1 & -1.205138 & 0.007362 & Down \\
\hline TC01004748.hg.1 & -1.265677 & 0.007451 & Down \\
\hline TC11001732.hg.1 & -1.382737 & 0.007461 & Down \\
\hline TC07002298.hg.1 & -1.229053 & 0.007471 & Down \\
\hline TC02000198.hg.1 & -1.241303 & 0.007479 & Down \\
\hline TC19000822.hg.1 & -1.291223 & 0.007487 & Down \\
\hline TC05002943.hg.1 & -1.222683 & 0.00749 & Down \\
\hline
\end{tabular}


Table 2 (continued)

\begin{tabular}{llll}
\hline Probe set ID & Fold change & p-value & Gene feature \\
\hline TC05000859.hg.1 & -1.310438 & 0.007529 & Down \\
TC01006140.hg.1 & -1.291211 & 0.00756 & Down \\
TC02001517.hg.1 & -1.226001 & 0.007575 & Down \\
TC02001732.hg.1 & -1.211515 & 0.007601 & Down \\
TC17002605.hg.1 & -1.203515 & 0.007619 & Down \\
TC02004353.hg.1 & -1.446996 & 0.007622 & Down \\
\hline
\end{tabular}

\begin{tabular}{llll}
\hline Probe set ID & Fold change & p-value & Gene feature \\
\hline
\end{tabular}

(C) Genes up/down-regulated in non-purified lung $\mathrm{TCS}^{\mathrm{SV} 40}$ compared with nonpurified primary lung TCS

\begin{tabular}{|c|c|c|c|}
\hline TC03003114.hg.1 & 4.734104 & $2.70 E-05$ & Up \\
\hline TC17001801.hg.1 & -2.581599 & $3.00 E-05$ & Down \\
\hline TC11002894.hg.1 & 3.769187 & $3.20 E-05$ & Up \\
\hline TC04000160.hg.1 & -2.019509 & $3.50 E-05$ & Down \\
\hline TC20000952.hg.1 & -2.001725 & $3.80 E-05$ & Down \\
\hline TC04002890.hg.1 & 2.318185 & $4.00 E-05$ & Up \\
\hline TC11003109.hg.1 & 5.927567 & $4.30 E-05$ & Up \\
\hline TC02000205.hg.1 & -1.864365 & $4.50 E-05$ & Down \\
\hline TC12000558.hg.1 & -1.77743 & $4.80 E-05$ & Down \\
\hline TC09000971.hg.1 & -1.924593 & $5.10 E-05$ & Down \\
\hline TCOX001292.hg.1 & -3.110776 & $5.30 E-05$ & Down \\
\hline TC08000385.hg.1 & -1.758147 & $5.60 \mathrm{E}-05$ & Down \\
\hline TC02004814.hg.1 & -1.730004 & $5.80 \mathrm{E}-05$ & Down \\
\hline TC06001978.hg.1 & -2.037057 & $6.10 E-05$ & Down \\
\hline TC12001286.hg.1 & -1.823703 & $6.40 E-05$ & Down \\
\hline TC08000302.hg.1 & -2.018038 & $6.60 E-05$ & Down \\
\hline TC07001784.hg.1 & -1.938506 & $6.90 E-05$ & Down \\
\hline TC12000927.hg.1 & -2.156997 & $7.10 E-05$ & Down \\
\hline TC15000452.hg.1 & -2.013654 & $7.40 E-05$ & Down \\
\hline TC11002382.hg.1 & -3.896927 & $7.70 \mathrm{E}-05$ & Down \\
\hline TC02001940.hg.1 & -1.982875 & $7.90 \mathrm{E}-05$ & Down \\
\hline TC09001648.hg.1 & -2.074788 & $8.20 E-05$ & Down \\
\hline TC02001602.hg.1 & -1.697784 & $8.40 E-05$ & Down \\
\hline TC06000639.hg.1 & -2.003858 & $8.70 E-05$ & Down \\
\hline TC04002560.hg.1 & 2.166732 & $9.00 \mathrm{E}-05$ & Up \\
\hline TC17001773.hg.1 & -1.582128 & $9.20 E-05$ & Down \\
\hline TC13000783.hg.1 & -2.527727 & $9.50 E-05$ & Down \\
\hline TCOX001624.hg.1 & -1.937361 & $9.70 \mathrm{E}-05$ & Down \\
\hline TC05001593.hg.1 & -1.926476 & $1.00 E-04$ & Down \\
\hline TC19001567.hg.1 & 1.692676 & 0.000103 & Up \\
\hline TC02002456.hg.1 & -1.806913 & 0.000105 & Down \\
\hline TC08002421.hg.1 & -2.163126 & 0.000108 & Down \\
\hline TC09001898.hg.1 & -1.436061 & 0.00011 & Down \\
\hline TC0X000450.hg.1 & -1.484801 & 0.000113 & Down \\
\hline TC02003744.hg.1 & 1.585428 & 0.000116 & Up \\
\hline TC07002463.hg.1 & 1.777811 & 0.000118 & Up \\
\hline TC07001655.hg.1 & -1.503467 & 0.000121 & Down \\
\hline TC22000156.hg.1 & 1.898289 & 0.000123 & Up \\
\hline TC16000648.hg.1 & -2.164873 & 0.000126 & Down \\
\hline TC04002642.hg.1 & -1.587573 & 0.000129 & Down \\
\hline TC16001289.hg.1 & -1.766618 & 0.000131 & Down \\
\hline TC06000568.hg.1 & -1.73117 & 0.000134 & Down \\
\hline TC20000650.hg.1 & -1.835321 & 0.000136 & Down \\
\hline
\end{tabular}

Table 2 (continued)

\begin{tabular}{|c|c|c|c|}
\hline Probe set ID & Fold change & p-value & Gene feature \\
\hline TCOX001889.hg.1 & 1.580745 & 0.000139 & Up \\
\hline TC04001945.hg.1 & -1.643053 & 0.000142 & Down \\
\hline TC11001010.hg.1 & -1.603866 & 0.000144 & Down \\
\hline TC03001841.hg.1 & -1.766003 & 0.000147 & Down \\
\hline TC05003313.hg.1 & 1.920567 & 0.000149 & Up \\
\hline TC05001142.hg.1 & -1.371777 & 0.000152 & Down \\
\hline TC02000396.hg.1 & -1.829949 & 0.000155 & Down \\
\hline TC12000231.hg.1 & -1.550803 & 0.000157 & Down \\
\hline TC17000491.hg.1 & -1.592587 & 0.00016 & Down \\
\hline TC0X001430.hg.1 & -1.820415 & 0.000162 & Down \\
\hline TC16002030.hg.1 & -1.524415 & 0.000165 & Down \\
\hline TC02001836.hg.1 & -1.49041 & 0.000168 & Down \\
\hline TC07002531.hg.1 & 1.715119 & 0.00017 & Up \\
\hline TC17002479.hg.1 & 1.76047 & 0.000173 & Up \\
\hline TC04001635.hg.1 & -2.303785 & 0.000175 & Down \\
\hline TC01003308.hg.1 & -1.900128 & 0.000178 & Down \\
\hline TC08002253.hg.1 & -2.893756 & 0.000181 & Down \\
\hline TC06000705.hg.1 & -1.731076 & 0.000183 & Down \\
\hline TC08000801.hg.1 & -1.729942 & 0.000186 & Down \\
\hline TC15001184.hg.1 & -1.439815 & 0.000188 & Down \\
\hline TCOX001410.hg.1 & -1.581381 & 0.000191 & Down \\
\hline TC16001066.hg.1 & -1.31385 & 0.000194 & Down \\
\hline TC0X001822.hg.1 & 1.439398 & 0.000196 & Up \\
\hline TC22001175.hg.1 & -1.542513 & 0.000199 & Down \\
\hline TC05002512.hg.1 & -1.48484 & 0.000201 & Down \\
\hline TC12001449.hg.1 & -1.863709 & 0.000204 & Down \\
\hline TC01001501.hg.1 & -1.466269 & 0.000207 & Down \\
\hline TC20000373.hg.1 & -1.596942 & 0.000209 & Down \\
\hline TC01003990.hg.1 & -1.514826 & 0.000212 & Down \\
\hline TC09000096.hg.1 & -1.937731 & 0.000214 & Down \\
\hline TC06000824.hg.1 & -1.586137 & 0.000217 & Down \\
\hline TC10002723.hg.1 & -1.693797 & 0.00022 & Down \\
\hline TC06004042.hg.1 & -1.463012 & 0.000222 & Down \\
\hline TC19000767.hg.1 & -1.80746 & 0.000225 & Down \\
\hline TC15001604.hg.1 & -1.573292 & 0.000227 & Down \\
\hline TCOX000985.hg.1 & -2.24906 & 0.00023 & Down \\
\hline TC0X001210.hg.1 & -1.648953 & 0.000233 & Down \\
\hline TC08002510.hg.1 & 2.254553 & 0.000235 & Up \\
\hline TC12000633.hg.1 & -1.764414 & 0.000238 & Down \\
\hline TC06003416.hg.1 & -1.389758 & 0.00024 & Down \\
\hline TC09000963.hg.1 & -1.4781 & 0.000243 & Down \\
\hline TC15001326.hg.1 & -1.537491 & 0.000246 & Down \\
\hline TC01004763.hg.1 & 1.400232 & 0.000248 & Up \\
\hline TC12002858.hg.1 & 1.534662 & 0.000251 & Up \\
\hline TC05002279.hg.1 & -1.509417 & 0.000253 & Down \\
\hline TCOX001136.hg.1 & -1.444487 & 0.000256 & Down \\
\hline TC08000652.hg.1 & -1.577232 & 0.000259 & Down \\
\hline TC11002486.hg.1 & -1.651639 & 0.000261 & Down \\
\hline TC07003059.hg.1 & 1.417719 & 0.000264 & Up \\
\hline TC02000955.hg.1 & -1.666162 & 0.000266 & Down \\
\hline TC14001892.hg.1 & -1.606681 & 0.000269 & Down \\
\hline TC17001656.hg.1 & -1.472658 & 0.000272 & Down \\
\hline
\end{tabular}


Table 2 (continued)

\begin{tabular}{|c|c|c|c|}
\hline Probe set ID & Fold change & p-value & Gene feature \\
\hline TC06002227.hg.1 & -1.627176 & 0.000274 & Down \\
\hline TC05000998.hg.1 & -1.408795 & 0.000277 & Down \\
\hline TC09001602.hg.1 & -1.887078 & 0.000279 & Down \\
\hline TC09001534.hg.1 & -1.467576 & 0.000282 & Down \\
\hline TC02004275.hg.1 & -1.320789 & 0.000285 & Down \\
\hline TC17000728.hg.1 & -2.065452 & 0.000287 & Down \\
\hline TC06000725.hg.1 & 1.440025 & 0.00029 & Up \\
\hline TC10002919.hg.1 & -1.600614 & 0.000292 & Down \\
\hline TC12000805.hg.1 & -1.56655 & 0.000295 & Down \\
\hline TC10000686.hg.1 & -1.469338 & 0.000298 & Down \\
\hline TC0X001158.hg.1 & -1.926968 & $3.00 E-04$ & Down \\
\hline TC09001936.hg.1 & -1.453724 & 0.000303 & Down \\
\hline TC16001498.hg.1 & 1.666085 & 0.000305 & Up \\
\hline TC12002493.hg.1 & 1.355038 & 0.000308 & Up \\
\hline TC10000439.hg.1 & -1.562003 & 0.000311 & Down \\
\hline TCOX001411.hg.1 & -1.587704 & 0.000313 & Down \\
\hline TC10000866.hg.1 & -1.575569 & 0.000318 & Down \\
\hline TC05002034.hg.1 & -1.483066 & 0.000321 & Down \\
\hline TCOY000064.hg.1 & 1.420454 & 0.000325 & Up \\
\hline TCOY000173.hg.1 & 1.420454 & 0.000325 & Up \\
\hline TC08001864.hg.1 & 2.861562 & 0.000329 & Up \\
\hline TC19000163.hg.1 & -1.609154 & 0.000331 & Down \\
\hline TC09000516.hg.1 & -1.559053 & 0.000334 & Down \\
\hline TC14002059.hg.1 & 1.551644 & 0.000337 & Up \\
\hline TC11002784.hg.1 & 1.820896 & 0.000339 & Up \\
\hline TC07002052.hg.1 & -1.561394 & 0.000342 & Down \\
\hline TC03002116.hg.1 & -1.474893 & 0.000344 & Down \\
\hline TC1 1002476.hg.1 & -1.57355 & 0.000347 & Down \\
\hline TC06002303.hg.1 & -1.518545 & 0.00035 & Down \\
\hline TC14001731.hg.1 & 1.403751 & 0.000352 & Up \\
\hline TC04002294.hg.1 & -1.448062 & 0.000355 & Down \\
\hline TC01003510.hg.1 & -1.493469 & 0.000357 & Down \\
\hline TC05001562.hg.1 & -1.537085 & 0.00036 & Down \\
\hline TC01002976.hg.1 & -1.566265 & 0.000362 & Down \\
\hline TC02001432.hg.1 & -1.55349 & 0.000365 & Down \\
\hline TC16001854.hg.1 & -1.350821 & 0.000368 & Down \\
\hline TC21000698.hg.1 & -1.401198 & 0.00037 & Down \\
\hline TC03000195.hg.1 & -1.616867 & 0.000373 & Down \\
\hline TC06001651.hg.1 & -1.50443 & 0.000375 & Down \\
\hline TC06001365.hg.1 & -1.809445 & 0.000378 & Down \\
\hline TC07001706.hg.1 & -1.618734 & 0.000382 & Down \\
\hline TC07001709.hg.1 & -1.618734 & 0.000382 & Down \\
\hline TC15002247.hg.1 & 1.354504 & 0.000386 & Up \\
\hline TC21000931.hg.1 & -1.420416 & 0.000391 & Down \\
\hline TC04002443.hg.1 & -1.551184 & 0.000394 & Down \\
\hline TC09000586.hg.1 & -1.554678 & 0.000396 & Down \\
\hline TC14000620.hg.1 & -1.494772 & 0.000399 & Down \\
\hline TC06002402.hg.1 & 1.646644 & 0.000401 & Up \\
\hline TC12000088.hg.1 & 1.444149 & 0.000404 & Up \\
\hline TC01002798.hg.1 & -1.895656 & 0.000407 & Down \\
\hline TC05002818.hg.1 & -1.513562 & 0.000409 & Down \\
\hline TC03001056.hg.1 & -1.606915 & 0.000412 & Down \\
\hline
\end{tabular}

Table 2 (continued)

\begin{tabular}{|c|c|c|c|}
\hline Probe set ID & Fold change & p-value & Gene feature \\
\hline TC01002746.hg.1 & -1.453698 & 0.000414 & Down \\
\hline TC02003041.hg.1 & -1.421703 & 0.000417 & Down \\
\hline TC04000811.hg.1 & -1.391548 & 0.00042 & Down \\
\hline TC09002304.hg.1 & -1.275207 & 0.000422 & Down \\
\hline TC05002800.hg.1 & -1.443218 & 0.000425 & Down \\
\hline TC03003057.hg.1 & 1.435368 & 0.000427 & Up \\
\hline TC11002810.hg.1 & 1.338827 & 0.00043 & Up \\
\hline TC08002059.hg.1 & 1.653874 & 0.000433 & Up \\
\hline TCOX002006.hg.1 & -1.347272 & 0.000435 & Down \\
\hline TC04001524.hg.1 & -1.559985 & 0.000438 & Down \\
\hline TC22001053.hg.1 & -1.465136 & 0.00044 & Down \\
\hline TC05000688.hg.1 & -1.557631 & 0.000443 & Down \\
\hline TC08002396.hg.1 & -1.266228 & 0.000446 & Down \\
\hline TC03001571.hg.1 & -1.575772 & 0.000448 & Down \\
\hline TC09002074.hg.1 & 1.663248 & 0.000451 & Up \\
\hline TC18000864.hg.1 & 1.491055 & 0.000453 & Up \\
\hline TC02001429.hg.1 & -1.512663 & 0.000456 & Down \\
\hline TC22000400.hg.1 & -1.474029 & 0.000459 & Down \\
\hline TC04001675.hg.1 & 1.523103 & 0.000461 & Up \\
\hline TC05000183.hg.1 & -1.591351 & 0.000466 & Down \\
\hline TC08000450.hg.1 & -1.754444 & 0.000469 & Down \\
\hline TC22001417.hg.1 & -1.575065 & 0.000477 & Down \\
\hline TC16001991.hg.1 & -1.30884 & 0.000479 & Down \\
\hline TC02004784.hg.1 & -1.291479 & 0.000482 & Down \\
\hline TC12003094.hg.1 & -1.450031 & 0.000487 & Down \\
\hline TC05002434.hg.1 & 1.258242 & 0.00049 & Up \\
\hline TC06002096.hg.1 & -1.459176 & 0.000492 & Down \\
\hline TC09000471.hg.1 & -1.399173 & 0.000495 & Down \\
\hline TC08002500.hg.1 & -1.277475 & 0.000498 & Down \\
\hline TC21000887.hg.1 & 1.340516 & $5.00 E-04$ & Up \\
\hline TC04001473.hg.1 & -1.527189 & 0.000503 & Down \\
\hline TC01000642.hg.1 & -1.492463 & 0.000505 & Down \\
\hline TC07001788.hg.1 & -1.686158 & 0.000508 & Down \\
\hline TC02003970.hg.1 & -1.440374 & 0.000511 & Down \\
\hline TC07000478.hg.1 & -1.476748 & 0.000513 & Down \\
\hline TC01005360.hg.1 & 1.315026 & 0.000516 & Up \\
\hline TC08002335.hg.1 & -1.716035 & 0.000518 & Down \\
\hline TC14002181.hg.1 & -1.260915 & 0.000521 & Down \\
\hline TC01002620.hg.1 & -1.443838 & 0.000526 & Down \\
\hline TC16001156.hg.1 & -1.403474 & 0.000531 & Down \\
\hline TC11001288.hg.1 & -1.336498 & 0.000534 & Down \\
\hline TC12003194.hg.1 & -1.56187 & 0.000537 & Down \\
\hline TCOX002258.hg.1 & -1.47544 & 0.000539 & Down \\
\hline TC05001001.hg.1 & -1.403589 & 0.000542 & Down \\
\hline TC04002346.hg.1 & 1.340139 & 0.000544 & Up \\
\hline TC06002606.hg.1 & -1.808218 & 0.000547 & Down \\
\hline TC14000667.hg.1 & 1.35603 & 0.00055 & Up \\
\hline TC03003257.hg.1 & -1.541547 & 0.000552 & Down \\
\hline TC13001029.hg.1 & 1.266449 & 0.000555 & Up \\
\hline TC01006195.hg.1 & -1.266774 & 0.000557 & Down \\
\hline TC12001995.hg.1 & -1.530295 & 0.00056 & Down \\
\hline TC12001434.hg.1 & -1.326576 & 0.000563 & Down \\
\hline
\end{tabular}


Table 2 (continued)

\begin{tabular}{|c|c|c|c|}
\hline Probe set ID & Fold change & p-value & Gene feature \\
\hline TC10000232.hg.1 & -1.308551 & 0.000565 & Down \\
\hline TC03003056.hg.1 & 1.356981 & 0.000568 & Up \\
\hline TC01006074.hg.1 & -1.330658 & 0.00057 & Down \\
\hline TC11003147.hg.1 & -1.269907 & 0.000573 & Down \\
\hline TC03002679.hg.1 & 1.353352 & 0.000576 & Up \\
\hline TC0X001094.hg.1 & -1.384223 & 0.000578 & Down \\
\hline TC09002823.hg.1 & 1.490093 & 0.000581 & Up \\
\hline TC13001025.hg.1 & 1.345303 & 0.000586 & Up \\
\hline TC09000043.hg.1 & 1.295164 & 0.000589 & Up \\
\hline TC10001021.hg.1 & -1.339644 & 0.000591 & Down \\
\hline TC12001052.hg.1 & -1.639168 & 0.000594 & Down \\
\hline TC04000338.hg.1 & -1.30348 & 0.000596 & Down \\
\hline TC02001799.hg.1 & -2.28736 & 0.000602 & Down \\
\hline TC14000739.hg.1 & 1.501284 & 0.000604 & Up \\
\hline TC04000508.hg.1 & -1.464186 & 0.000607 & Down \\
\hline TC09001812.hg.1 & -1.251376 & 0.000609 & Down \\
\hline TC08001514.hg.1 & -1.447493 & 0.000612 & Down \\
\hline TC02003364.hg.1 & -1.343783 & 0.000615 & Down \\
\hline TC0X001068.hg.1 & -1.274558 & 0.000617 & Down \\
\hline TC09001015.hg.1 & -1.353939 & 0.000622 & Down \\
\hline TC18000408.hg.1 & 1.509875 & 0.000625 & Up \\
\hline TC10001530.hg.1 & -1.256877 & 0.00063 & Down \\
\hline TC06003710.hg.1 & -1.416403 & 0.000633 & Down \\
\hline TC12002688.hg.1 & -1.639852 & 0.000635 & Down \\
\hline TC20000045.hg.1 & -1.459984 & 0.000638 & Down \\
\hline TC03002103.hg.1 & -1.492607 & 0.000641 & Down \\
\hline TC0X001762.hg.1 & -1.384395 & 0.000646 & Down \\
\hline TC01001291.hg.1 & 1.372502 & 0.000648 & Up \\
\hline TC09002148.hg.1 & -1.353651 & 0.000651 & Down \\
\hline TC05002688.hg.1 & -1.312714 & 0.000654 & Down \\
\hline TC19000423.hg.1 & -1.330548 & 0.000656 & Down \\
\hline TC10002884.hg.1 & 1.509065 & 0.000659 & Up \\
\hline TC04002813.hg.1 & 1.252444 & 0.000661 & Up \\
\hline TC18000381.hg.1 & -1.501444 & 0.000664 & Down \\
\hline TC01004281.hg.1 & -1.351776 & 0.000667 & Down \\
\hline TC11002492.hg.1 & -1.294008 & 0.000669 & Down \\
\hline TC21000149.hg.1 & -1.321876 & 0.000672 & Down \\
\hline TC04002187.hg.1 & 1.496304 & 0.000677 & Up \\
\hline TC09002847.hg.1 & -1.577697 & 0.00068 & Down \\
\hline TC09000642.hg.1 & -1.528996 & 0.000682 & Down \\
\hline TC12001447.hg.1 & -1.218994 & 0.000685 & Down \\
\hline TC01004750.hg.1 & -1.405462 & 0.000687 & Down \\
\hline TC01006068.hg.1 & -1.764285 & 0.000693 & Down \\
\hline TC15001808.hg.1 & 1.40097 & 0.000698 & Up \\
\hline TC01002633.hg.1 & -1.562471 & 7.00E-04 & Down \\
\hline TC19002149.hg.1 & 1.387591 & 0.000703 & Up \\
\hline TC02002581.hg.1 & -1.319171 & 0.000706 & Down \\
\hline TC15002417.hg.1 & -1.354206 & 0.000708 & Down \\
\hline TC18000603.hg.1 & 1.83788 & 0.000711 & Up \\
\hline TC02003574.hg.1 & -1.264549 & 0.000713 & Down \\
\hline TC09000458.hg.1 & -1.457816 & 0.000716 & Down \\
\hline TC02003914.hg.1 & -1.539912 & 0.000721 & Down \\
\hline TC08000285.hg.1 & -1.530341 & 0.000724 & Down \\
\hline
\end{tabular}

Table 2 (continued)

\begin{tabular}{|c|c|c|c|}
\hline Probe set ID & Fold change & p-value & Gene feature \\
\hline TC01005268.hg.1 & 1.791888 & 0.000726 & Up \\
\hline TC02001366.hg.1 & -1.401387 & 0.000729 & Down \\
\hline TC04001384.hg.1 & 1.371085 & 0.000731 & Up \\
\hline TC15001301.hg.1 & 1.410236 & 0.000734 & Up \\
\hline TC16002013.hg.1 & -1.607586 & 0.000739 & Down \\
\hline TC09000907.hg.1 & -1.304972 & 0.000742 & Down \\
\hline TC21000483.hg.1 & -1.526928 & 0.000744 & Down \\
\hline TC16001359.hg.1 & 1.255541 & 0.00075 & Up \\
\hline TC02002970.hg.1 & -1.479739 & 0.000755 & Down \\
\hline TC07000690.hg.1 & -1.482383 & 0.000757 & Down \\
\hline TC12001768.hg.1 & -1.570223 & 0.00076 & Down \\
\hline TC01005811.hg.1 & 1.326118 & 0.000763 & Up \\
\hline TC03001366.hg.1 & -1.272926 & 0.000765 & Down \\
\hline TC14000809.hg.1 & -1.304326 & 0.000768 & Down \\
\hline TC10001432.hg.1 & -1.399969 & 0.000773 & Down \\
\hline TC08000954.hg.1 & -1.3347 & 0.000778 & Down \\
\hline TC07002238.hg.1 & 1.338154 & 0.000781 & Up \\
\hline TC17001691.hg.1 & -1.285264 & 0.000783 & Down \\
\hline TC03000629.hg.1 & -1.661071 & 0.000786 & Down \\
\hline TC01003152.hg.1 & 1.470629 & 0.000789 & Up \\
\hline TC05001849.hg.1 & -1.23812 & 0.000791 & Down \\
\hline TC0X002282.hg.1 & -1.368206 & 0.000794 & Down \\
\hline TC07001801.hg.1 & 1.645383 & 0.000796 & Up \\
\hline TC16000919.hg.1 & -1.28532 & 0.000799 & Down \\
\hline TC16001996.hg.1 & -1.42464 & 0.000802 & Down \\
\hline TC15000819.hg.1 & -1.492594 & 0.000804 & Down \\
\hline TC07001099.hg.1 & -1.282727 & 0.000807 & Down \\
\hline TC01005416.hg.1 & 1.335933 & 0.000809 & Up \\
\hline TC08001082.hg.1 & -1.327711 & 0.000812 & Down \\
\hline TC09002810.hg.1 & -1.337917 & 0.000815 & Down \\
\hline TC15000346.hg.1 & 1.616409 & 0.00082 & Up \\
\hline TCOX000483.hg.1 & 1.311803 & 0.000822 & Up \\
\hline TCOX001733.hg.1 & 1.453339 & 0.000825 & Up \\
\hline TC01001142.hg.1 & 1.560006 & 0.000828 & Up \\
\hline TC12001702.hg.1 & -1.329841 & 0.00083 & Down \\
\hline TC01002339.hg.1 & -1.416764 & 0.000833 & Down \\
\hline TC12001455.hg.1 & -1.47722 & 0.000835 & Down \\
\hline TC02004623.hg.1 & -1.459571 & 0.000841 & Down \\
\hline TC08002093.hg.1 & -1.281485 & 0.000843 & Down \\
\hline TC01003671.hg.1 & -1.388896 & 0.000846 & Down \\
\hline TC09002037.hg.1 & -1.275106 & 0.000848 & Down \\
\hline TC15002699.hg.1 & 1.23712 & 0.000851 & Up \\
\hline TC09000060.hg.1 & -1.327162 & 0.000854 & Down \\
\hline TC08000411.hg.1 & -1.220223 & 0.000859 & Down \\
\hline TC03002290.hg.1 & -1.255338 & 0.000861 & Down \\
\hline TC02001844.hg.1 & 1.372199 & 0.000869 & Up \\
\hline TC15002646.hg.1 & 1.313486 & 0.000874 & Up \\
\hline TC12000278.hg.1 & -1.226492 & 0.000877 & Down \\
\hline TC14001143.hg.1 & -1.298709 & 0.000882 & Down \\
\hline TC04000543.hg.1 & 1.515491 & 0.000885 & Up \\
\hline TC04000223.hg.1 & -1.409422 & 0.000887 & Down \\
\hline TC20001277.hg.1 & 1.302102 & 0.00089 & Up \\
\hline
\end{tabular}


Table 2 (continued)

\begin{tabular}{|c|c|c|c|}
\hline Probe set ID & Fold change & p-value & Gene feature \\
\hline TC0X000283.hg.1 & -1.437052 & 0.000893 & Down \\
\hline TC17001310.hg.1 & -1.246777 & 0.000895 & Down \\
\hline TC05002436.hg.1 & -1.405807 & $9.00 \mathrm{E}-04$ & Down \\
\hline TC21000762.hg.1 & 1.418955 & 0.000906 & Up \\
\hline TC01002467.hg.1 & -1.36755 & 0.000908 & Down \\
\hline TC19002431.hg.1 & -1.272804 & 0.000913 & Down \\
\hline TC08000259.hg.1 & -1.366957 & 0.000916 & Down \\
\hline TC04002100.hg.1 & -1.333622 & 0.000932 & Down \\
\hline TC08000621.hg.1 & -1.330254 & 0.000937 & Down \\
\hline TC07002774.hg.1 & -1.220957 & 0.000942 & Down \\
\hline TC11000031.hg.1 & -1.301394 & 0.000945 & Down \\
\hline TC05001757.hg.1 & -1.61845 & 0.000947 & Down \\
\hline TC15002177.hg.1 & 1.216648 & 0.00095 & Up \\
\hline TC01004614.hg.1 & -1.232646 & 0.000955 & Down \\
\hline TC11003389.hg.1 & -1.289742 & 0.000958 & Down \\
\hline TC02002711.hg.1 & -1.307176 & 0.00096 & Down \\
\hline TC02003138.hg.1 & 1.406275 & 0.000963 & Up \\
\hline TC07000147.hg.1 & -1.360339 & 0.000965 & Down \\
\hline TC21000690.hg.1 & -1.263198 & 0.000968 & Down \\
\hline TC03003246.hg.1 & 1.264019 & 0.000971 & Up \\
\hline TC06002340.hg.1 & -1.442259 & 0.000976 & Down \\
\hline TC07000879.hg.1 & 1.459915 & 0.000978 & Up \\
\hline TC02002957.hg.1 & -1.235958 & 0.000991 & Down \\
\hline TC09002317.hg.1 & -1.249176 & 0.000994 & Down \\
\hline TC01004251.hg.1 & 1.286817 & 0.000999 & Up \\
\hline TC10000250.hg.1 & -1.295565 & 0.001002 & Down \\
\hline TC12000578.hg.1 & 1.229407 & 0.00101 & Up \\
\hline TC03003258.hg.1 & 1.233627 & 0.001012 & Up \\
\hline TC06004031.hg.1 & -1.414967 & 0.001015 & Down \\
\hline TC06001862.hg.1 & -1.33761 & 0.001017 & Down \\
\hline TC05001595.hg.1 & -1.371588 & 0.001023 & Down \\
\hline TC11000282.hg.1 & -1.382875 & 0.001028 & Down \\
\hline TC08001583.hg.1 & -1.478615 & 0.00103 & Down \\
\hline TC01004440.hg.1 & -1.297319 & 0.001033 & Down \\
\hline TC12000238.hg.1 & -1.399591 & 0.001036 & Down \\
\hline TC07000119.hg.1 & -1.27944 & 0.001043 & Down \\
\hline TC01006051.hg.1 & -1.256434 & 0.001046 & Down \\
\hline TC02004706.hg.1 & 1.310009 & 0.001056 & Up \\
\hline TC02001302.hg.1 & -1.505454 & 0.001059 & Down \\
\hline TC07000115.hg.1 & -1.22225 & 0.001062 & Down \\
\hline TC03002003.hg.1 & -1.334984 & 0.001067 & Down \\
\hline TC17000637.hg.1 & 1.465593 & 0.001072 & Up \\
\hline TC01002594.hg.1 & -1.510462 & 0.001075 & Down \\
\hline TC12000589.hg.1 & -1.365986 & 0.001077 & Down \\
\hline TC22001135.hg.1 & -1.234824 & 0.00108 & Down \\
\hline TC09002305.hg.1 & 1.258887 & 0.001082 & Up \\
\hline TC17002700.hg.1 & -1.309071 & 0.001085 & Down \\
\hline TC10002643.hg.1 & 1.328739 & 0.001095 & Up \\
\hline TC01000612.hg.1 & -1.316806 & 0.001098 & Down \\
\hline TC09002594.hg.1 & 1.380711 & 0.0011 & Up \\
\hline TCOY000302.hg.1 & -1.259031 & 0.001107 & Down \\
\hline TCOY000304.hg.1 & -1.259031 & 0.001107 & Down \\
\hline
\end{tabular}

Table 2 (continued)

\begin{tabular}{|c|c|c|c|}
\hline Probe set ID & Fold change & $p$-value & Gene feature \\
\hline TC04000571.hg.1 & -1.451125 & 0.001113 & Down \\
\hline TC02002390.hg.1 & -1.333276 & 0.001116 & Down \\
\hline TC18000785.hg.1 & -1.282574 & 0.001119 & Down \\
\hline TC07002321.hg.1 & 1.255896 & 0.001121 & Up \\
\hline TC09001461.hg.1 & -1.450346 & 0.001126 & Down \\
\hline TC07002353.hg.1 & -1.289742 & 0.001129 & Down \\
\hline TC08000734.hg.1 & -1.225764 & 0.001132 & Down \\
\hline TC07001869.hg.1 & -1.64831 & 0.001137 & Down \\
\hline TC03000531.hg.1 & 1.33812 & 0.001142 & Up \\
\hline TC01002397.hg.1 & -1.297705 & 0.001145 & Down \\
\hline TCOY000124.hg.1 & 1.235109 & 0.001147 & Up \\
\hline TC09001504.hg.1 & -1.327742 & 0.00115 & Down \\
\hline TC07000516.hg.1 & 1.31773 & 0.001152 & Up \\
\hline TC22001201.hg.1 & -2.532162 & 0.00116 & Down \\
\hline TC19000785.hg.1 & -1.597258 & 0.001163 & Down \\
\hline TC08001887.hg.1 & 1.247089 & 0.001165 & Up \\
\hline TC10002136.hg.1 & -1.2106 & 0.001171 & Down \\
\hline TC02001567.hg.1 & -1.384195 & 0.001176 & Down \\
\hline TC09002851.hg.1 & -1.340312 & 0.001178 & Down \\
\hline TC21000511.hg.1 & 1.251439 & 0.001181 & Up \\
\hline TC20000751.hg.1 & -1.320938 & 0.001184 & Down \\
\hline TC21000719.hg.1 & -1.268148 & 0.001189 & Down \\
\hline TC04000456.hg.1 & -1.320603 & 0.001191 & Down \\
\hline TC15001493.hg.1 & -1.227503 & 0.001199 & Down \\
\hline TC12002442.hg.1 & -1.376817 & 0.001207 & Down \\
\hline TC03002939.hg.1 & 1.251726 & 0.001212 & Up \\
\hline TC03001734.hg.1 & 1.396213 & 0.001215 & Up \\
\hline TC12000588.hg.1 & -1.333007 & 0.001223 & Down \\
\hline TC21000447.hg.1 & -1.353397 & 0.001225 & Down \\
\hline TC07000702.hg.1 & -1.47349 & 0.001228 & Down \\
\hline TC20000413.hg.1 & -1.2025 & 0.001233 & Down \\
\hline TC18000406.hg.1 & 1.310188 & 0.001236 & Up \\
\hline TC04000366.hg.1 & 1.460437 & 0.001241 & Up \\
\hline TC03002319.hg.1 & -1.211798 & 0.001246 & Down \\
\hline TC07000882.hg.1 & -1.465665 & 0.001256 & Down \\
\hline TC11001732.hg.1 & -1.430354 & 0.001262 & Down \\
\hline TC11003052.hg.1 & 1.216234 & 0.001264 & Up \\
\hline TC06000255.hg.1 & 1.246413 & 0.001267 & Up \\
\hline TC02003743.hg.1 & 1.3116 & 0.001272 & Up \\
\hline TCOM000020.hg.1 & -1.462929 & 0.001275 & Down \\
\hline TC03002654.hg.1 & 1.460058 & 0.001282 & Up \\
\hline TC20001679.hg.1 & 1.583808 & 0.001285 & Up \\
\hline TC01001972.hg.1 & 1.328821 & 0.001288 & Up \\
\hline TC02000005.hg.1 & -1.220133 & 0.00129 & Down \\
\hline TC17001438.hg.1 & -1.37931 & 0.001298 & Down \\
\hline TC07001428.hg.1 & -1.277175 & 0.001301 & Down \\
\hline TC11000655.hg.1 & -1.292662 & 0.001308 & Down \\
\hline TC09002024.hg.1 & 1.331597 & 0.001319 & Up \\
\hline TC01003906.hg.1 & -1.246181 & 0.001324 & Down \\
\hline TC03001758.hg.1 & -1.335816 & 0.001327 & Down \\
\hline TC11003065.hg.1 & -1.28471 & 0.001329 & Down \\
\hline
\end{tabular}


Table 2 (continued)

\begin{tabular}{|c|c|c|c|}
\hline Probe set ID & Fold change & p-value & Gene feature \\
\hline TC22001002.hg.1 & -1.300797 & 0.001337 & Down \\
\hline TC20000993.hg.1 & 1.406397 & 0.001342 & Up \\
\hline TC02003376.hg.1 & 1.22424 & 0.001345 & Up \\
\hline TC17001702.hg.1 & -1.355539 & 0.001347 & Down \\
\hline TCOX001193.hg.1 & 1.492178 & 0.001358 & Up \\
\hline TC02002904.hg.1 & -1.387512 & 0.00136 & Down \\
\hline TC02001431.hg.1 & -1.260897 & 0.001363 & Down \\
\hline TC05000214.hg.1 & -1.320326 & 0.001366 & Down \\
\hline TC11003186.hg.1 & 1.239331 & 0.001371 & Up \\
\hline TC12003071.hg.1 & -1.273357 & 0.001379 & Down \\
\hline TC11002465.hg.1 & -1.301587 & 0.001381 & Down \\
\hline TC02001721.hg.1 & -1.243881 & 0.001384 & Down \\
\hline TC03001672.hg.1 & -1.312188 & 0.001386 & Down \\
\hline TC04001562.hg.1 & 1.448978 & 0.001397 & Up \\
\hline TC11002930.hg.1 & 1.32439 & 0.001399 & Up \\
\hline TC01005621.hg.1 & -1.346925 & 0.001402 & Down \\
\hline TC14000912.hg.1 & -1.274825 & 0.001405 & Down \\
\hline TC12000990.hg.1 & -1.323786 & 0.001415 & Down \\
\hline TC01003976.hg.1 & 1.256443 & 0.001418 & Up \\
\hline TC16001253.hg.1 & -1.403981 & 0.001425 & Down \\
\hline TC20001202.hg.1 & 1.266515 & 0.001436 & Up \\
\hline TC16000168.hg.1 & -1.27072 & 0.001438 & Down \\
\hline TC09002408.hg.1 & 1.520922 & 0.001441 & Up \\
\hline TC16000573.hg.1 & -1.259628 & 0.001451 & Down \\
\hline TC15000227.hg.1 & -1.252319 & 0.001454 & Down \\
\hline TC10000082.hg.1 & -1.386938 & 0.001456 & Down \\
\hline TC04000854.hg.1 & -1.36653 & 0.001459 & Down \\
\hline TC09002413.hg.1 & -1.24335 & 0.001462 & Down \\
\hline TC17002577.hg.1 & -1.227651 & 0.001464 & Down \\
\hline TCOM000023.hg.1 & -1.325689 & 0.001467 & Down \\
\hline TC0X000584.hg.1 & 1.524739 & 0.001482 & Up \\
\hline TC17000782.hg.1 & -1.248164 & 0.001485 & Down \\
\hline TC01000565.hg.1 & -1.252749 & 0.00149 & Down \\
\hline TC06002722.hg.1 & -1.238586 & 0.001495 & Down \\
\hline TC05001279.hg.1 & -1.228112 & 0.001498 & Down \\
\hline TC05002924.hg.1 & -1.252361 & 0.001501 & Down \\
\hline TC01000711.hg.1 & -1.284644 & 0.001516 & Down \\
\hline TC02000092.hg.1 & -1.239683 & 0.001534 & Down \\
\hline TC07000209.hg.1 & -1.234204 & 0.001537 & Down \\
\hline TC07001848.hg.1 & -1.342223 & 0.001547 & Down \\
\hline TC13001179.hg.1 & -1.291746 & 0.00155 & Down \\
\hline TC15002627.hg.1 & -1.241372 & 0.001553 & Down \\
\hline TC03000245.hg.1 & -1.251682 & 0.001555 & Down \\
\hline TC11002051.hg.1 & -1.298163 & 0.001558 & Down \\
\hline TC01005264.hg.1 & -1.432014 & 0.001568 & Down \\
\hline TC13000269.hg.1 & -1.405203 & 0.001571 & Down \\
\hline TC05001668.hg.1 & -1.264735 & 0.001573 & Down \\
\hline TC02000188.hg.1 & -1.278172 & 0.001592 & Down \\
\hline TC20000564.hg.1 & -1.288599 & 0.001594 & Down \\
\hline TC02002994.hg.1 & -1.487671 & 0.001597 & Down \\
\hline TC07002487.hg.1 & -1.275774 & 0.001607 & Down \\
\hline TC17001587.hg.1 & -1.227915 & 0.00161 & Down \\
\hline
\end{tabular}

Table 2 (continued)

\begin{tabular}{|c|c|c|c|}
\hline Probe set ID & Fold change & p-value & Gene feature \\
\hline TC14000711.hg.1 & -1.272822 & 0.00162 & Down \\
\hline TC02003983.hg.1 & -1.242 & 0.001628 & Down \\
\hline TC05003265.hg.1 & -1.230554 & 0.001636 & Down \\
\hline TC14000850.hg.1 & -1.216954 & 0.001649 & Down \\
\hline TC08000062.hg.1 & -1.269982 & 0.001654 & Down \\
\hline TC02004175.hg.1 & -1.224219 & 0.001675 & Down \\
\hline TC06001872.hg.1 & -1.257336 & 0.00168 & Down \\
\hline TC08000176.hg.1 & -1.220994 & 0.001693 & Down \\
\hline TC15001480.hg.1 & -1.381133 & 0.001701 & Down \\
\hline TC03000233.hg.1 & -1.272324 & 0.001722 & Down \\
\hline TC09002445.hg.1 & -1.336146 & 0.001745 & Down \\
\hline TC08001621.hg.1 & -1.261265 & 0.001758 & Down \\
\hline TC09001799.hg.1 & -1.266496 & 0.001763 & Down \\
\hline TC12001128.hg.1 & -1.30415 & 0.001766 & Down \\
\hline TC01001028.hg.1 & -1.345191 & 0.001787 & Down \\
\hline TC05000708.hg.1 & -1.476725 & 0.001797 & Down \\
\hline TC04000307.hg.1 & -1.201551 & 0.001802 & Down \\
\hline TC04001876.hg.1 & -1.312985 & 0.001833 & Down \\
\hline TC02004514.hg.1 & -1.297988 & 0.001838 & Down \\
\hline TC12003114.hg.1 & -1.324803 & 0.001846 & Down \\
\hline TC12000184.hg.1 & -1.231818 & 0.001849 & Down \\
\hline TC12002387.hg.1 & -1.258686 & 0.001859 & Down \\
\hline TCOX000282.hg.1 & -1.45897 & 0.001862 & Down \\
\hline TC17001193.hg.1 & -1.316251 & 0.001867 & Down \\
\hline TC03001348.hg.1 & -1.255899 & 0.00187 & Down \\
\hline TC11002740.hg.1 & -1.257394 & 0.001872 & Down \\
\hline TC16000982.hg.1 & -1.234762 & 0.001875 & Down \\
\hline TC01004296.hg.1 & -1.287062 & 0.001877 & Down \\
\hline TC17002761.hg.1 & -1.320442 & 0.001883 & Down \\
\hline TC03000215.hg.1 & -1.209242 & 0.001885 & Down \\
\hline TC21000941.hg.1 & -1.285527 & 0.00189 & Down \\
\hline TC01005631.hg.1 & -1.230716 & 0.001903 & Down \\
\hline TC05002180.hg.1 & -1.262406 & 0.00194 & Down \\
\hline TC02001854.hg.1 & -1.397183 & 0.001945 & Down \\
\hline TC02001762.hg.1 & -1.306576 & 0.001953 & Down \\
\hline TC20001199.hg.1 & -1.647289 & 0.001963 & Down \\
\hline TC09001709.hg.1 & -1.554081 & 0.001968 & Down \\
\hline TC09001939.hg.1 & -1.294994 & 0.001987 & Down \\
\hline TCOX000279.hg.1 & -1.345356 & 0.001989 & Down \\
\hline TC0X000632.hg.1 & -1.266722 & 0.002023 & Down \\
\hline TC09000488.hg.1 & -1.235635 & 0.002028 & Down \\
\hline TC16001481.hg.1 & -1.280691 & 0.002031 & Down \\
\hline TC14000004.hg.1 & -1.297753 & 0.002036 & Down \\
\hline TC03000982.hg.1 & -1.296044 & 0.002044 & Down \\
\hline TC17001757.hg.1 & -1.218957 & 0.002049 & Down \\
\hline TC09002325.hg.1 & -1.326214 & 0.002062 & Down \\
\hline TC06000943.hg.1 & -1.305433 & 0.002067 & Down \\
\hline TC01003971.hg.1 & -1.348647 & 0.002091 & Down \\
\hline $\begin{array}{l}\text { TC6_cox_ } \\
\text { hap2000100.hg.1 }\end{array}$ & -1.554297 & 0.002098 & Down \\
\hline TC15001904.hg.1 & -1.252785 & 0.002106 & Down \\
\hline TC01002757.hg.1 & -1.351323 & 0.002109 & Down \\
\hline
\end{tabular}


Table 2 (continued)

\begin{tabular}{|c|c|c|c|}
\hline Probe set ID & Fold change & p-value & Gene feature \\
\hline TC09001332.hg.1 & -1.443525 & 0.002111 & Down \\
\hline TC10002850.hg.1 & -1.234143 & 0.002124 & Down \\
\hline TC04000005.hg.1 & -1.301943 & 0.002127 & Down \\
\hline TC01005454.hg.1 & -1.236656 & 0.00213 & Down \\
\hline TC06000102.hg.1 & -1.270327 & 0.002137 & Down \\
\hline TC10000369.hg.1 & -1.297064 & 0.00214 & Down \\
\hline TC05001222.hg.1 & -1.318072 & 0.002153 & Down \\
\hline TC14002139.hg.1 & -1.315965 & 0.002192 & Down \\
\hline TC12001777.hg.1 & -1.361163 & 0.002194 & Down \\
\hline TCOY000257.hg.1 & -1.300159 & 0.0022 & Down \\
\hline TC14000798.hg.1 & -1.51072 & 0.002202 & Down \\
\hline TC10001326.hg.1 & -1.235462 & 0.002213 & Down \\
\hline TC08001540.hg.1 & -1.313364 & 0.00222 & Down \\
\hline TC18000718.hg.1 & -1.313197 & 0.002226 & Down \\
\hline TC17002085.hg.1 & -1.303126 & 0.002249 & Down \\
\hline TC20001091.hg.1 & -1.214845 & 0.002262 & Down \\
\hline TC15001671.hg.1 & -1.222416 & 0.00227 & Down \\
\hline TC01003519.hg.1 & -1.227491 & 0.00228 & Down \\
\hline TC19001173.hg.1 & -1.504936 & 0.002293 & Down \\
\hline TC06003422.hg.1 & -1.26218 & 0.002306 & Down \\
\hline TC14001264.hg.1 & -1.271609 & 0.002314 & Down \\
\hline TC0X000986.hg.1 & -1.615057 & 0.002324 & Down \\
\hline TC10002901.hg.1 & -1.237562 & 0.00233 & Down \\
\hline TC11001264.hg.1 & -1.288498 & 0.002337 & Down \\
\hline TC14000795.hg.1 & -1.294836 & 0.002356 & Down \\
\hline TC05002063.hg.1 & -1.291118 & 0.002374 & Down \\
\hline TC11002064.hg.1 & -1.253136 & 0.002382 & Down \\
\hline TC17001939.hg.1 & -1.311247 & 0.002384 & Down \\
\hline TC02000413.hg.1 & -1.221091 & 0.002395 & Down \\
\hline TC08000956.hg.1 & -1.294681 & 0.002423 & Down \\
\hline TC05000526.hg.1 & -1.202821 & 0.002426 & Down \\
\hline TC02004253.hg.1 & -1.236233 & 0.002428 & Down \\
\hline TC01004790.hg.1 & -1.213559 & 0.002431 & Down \\
\hline TC02003570.hg.1 & -1.266677 & 0.002439 & Down \\
\hline TC04000311.hg.1 & -1.231832 & 0.002444 & Down \\
\hline TC19001288.hg.1 & -1.25999 & 0.002454 & Down \\
\hline TC14000697.hg.1 & -1.404857 & 0.002483 & Down \\
\hline TC09001077.hg.1 & -1.253924 & 0.002496 & Down \\
\hline TC21000650.hg.1 & -1.255534 & 0.002501 & Down \\
\hline TC07000862.hg.1 & -1.26199 & 0.002504 & Down \\
\hline TC09001024.hg.1 & -1.234413 & 0.002509 & Down \\
\hline TC03000889.hg.1 & -1.455333 & 0.002517 & Down \\
\hline TC04002712.hg.1 & -1.205148 & 0.002522 & Down \\
\hline TC11000172.hg.1 & -1.3994 & 0.00253 & Down \\
\hline TC22001095.hg.1 & -1.245068 & 0.002532 & Down \\
\hline TC17000164.hg.1 & -1.350111 & 0.002556 & Down \\
\hline TC02003220.hg.1 & -1.27533 & 0.002561 & Down \\
\hline TC02003240.hg.1 & -1.27617 & 0.002576 & Down \\
\hline TC01002756.hg.1 & -1.451337 & 0.002592 & Down \\
\hline TC04000671.hg.1 & -1.288484 & 0.002597 & Down \\
\hline TC11003125.hg.1 & -1.237544 & 0.002628 & Down \\
\hline TC08000224.hg.1 & -1.240388 & 0.002647 & Down \\
\hline
\end{tabular}

Table 2 (continued)

\begin{tabular}{|c|c|c|c|}
\hline Probe set ID & Fold change & p-value & Gene feature \\
\hline TC15002487.hg.1 & -1.375221 & 0.002649 & Down \\
\hline TC02004203.hg.1 & -1.328396 & 0.00267 & Down \\
\hline TC07002457.hg.1 & -1.2626 & 0.002699 & Down \\
\hline TC11000768.hg.1 & -1.278624 & 0.002704 & Down \\
\hline TC01001663.hg.1 & -1.240612 & 0.002719 & Down \\
\hline TC01000364.hg.1 & -1.295795 & 0.002722 & Down \\
\hline TC14001177.hg.1 & -1.215351 & 0.00273 & Down \\
\hline TC18000510.hg.1 & -1.339711 & 0.002743 & Down \\
\hline TC15001577.hg.1 & -1.296726 & 0.002748 & Down \\
\hline TC1 1002224.hg.1 & -1.418547 & 0.002761 & Down \\
\hline TC05002475.hg.1 & -1.29094 & 0.002769 & Down \\
\hline TC04001202.hg.1 & -1.267133 & 0.002777 & Down \\
\hline TC15001164.hg.1 & -1.256707 & 0.002784 & Down \\
\hline TC06002310.hg.1 & -1.205867 & 0.00281 & Down \\
\hline TC12000236.hg.1 & -1.234045 & 0.002813 & Down \\
\hline TC14000661.hg.1 & -1.277726 & 0.002826 & Down \\
\hline TC15002579.hg.1 & -1.217318 & 0.002849 & Down \\
\hline TC09000592.hg.1 & -1.249078 & 0.002857 & Down \\
\hline TC19000842.hg.1 & -1.275512 & 0.002873 & Down \\
\hline TC01001511.hg.1 & -1.274328 & 0.002878 & Down \\
\hline TC11000297.hg.1 & -1.333831 & 0.002883 & Down \\
\hline TC01005988.hg.1 & -1.399566 & 0.002899 & Down \\
\hline TC06000972.hg.1 & -1.307552 & 0.002912 & Down \\
\hline TC12000683.hg.1 & -1.31692 & 0.002919 & Down \\
\hline TC03000359.hg.1 & -1.2305 & 0.002938 & Down \\
\hline TC01006043.hg.1 & -1.294354 & 0.002956 & Down \\
\hline TC06001203.hg.1 & -1.210835 & 0.002977 & Down \\
\hline TC09002250.hg.1 & -1.30785 & 0.002979 & Down \\
\hline TC06003965.hg.1 & -1.25692 & 0.002982 & Down \\
\hline TC06001689.hg.1 & -1.271407 & 0.002995 & Down \\
\hline TC19000016.hg.1 & -1.280763 & 0.003005 & Down \\
\hline TC02000420.hg.1 & -1.290683 & 0.003016 & Down \\
\hline TC12001821.hg.1 & -1.233869 & 0.003031 & Down \\
\hline TC0X001706.hg.1 & -1.201138 & 0.003044 & Down \\
\hline TC02000115.hg.1 & -1.210348 & 0.003057 & Down \\
\hline TC02001877.hg.1 & -1.334891 & 0.00306 & Down \\
\hline TC21000155.hg. 1 & -1.319149 & 0.00307 & Down \\
\hline TC09002217.hg.1 & -1.202281 & 0.003075 & Down \\
\hline TC14000698.hg.1 & -1.271845 & 0.003078 & Down \\
\hline TC12002927.hg.1 & -1.241343 & 0.003083 & Down \\
\hline TC15002068.hg.1 & -1.295466 & 0.003091 & Down \\
\hline TC03000673.hg.1 & -1.219704 & 0.003117 & Down \\
\hline TC21000774.hg.1 & -1.304279 & 0.003122 & Down \\
\hline TC06002185.hg.1 & -1.21637 & 0.003138 & Down \\
\hline TC09000159.hg.1 & -1.452667 & 0.003143 & Down \\
\hline TC08002174.hg.1 & -1.299286 & 0.003174 & Down \\
\hline TC13000052.hg.1 & -1.220349 & 0.003226 & Down \\
\hline TC03002972.hg.1 & -1.308676 & 0.003244 & Down \\
\hline TC06003115.hg.1 & -1.208136 & 0.003278 & Down \\
\hline TC20000973.hg.1 & -1.239161 & 0.003296 & Down \\
\hline TC02004167.hg.1 & -1.20315 & 0.003299 & Down \\
\hline TC01005639.hg.1 & -1.239478 & 0.003304 & Down \\
\hline
\end{tabular}


Table 2 (continued)

\begin{tabular}{|c|c|c|c|}
\hline Probe set ID & Fold change & $p$-value & Gene feature \\
\hline TC03003205.hg.1 & -1.263929 & 0.003317 & Down \\
\hline TC03002458.hg.1 & -1.287128 & 0.003325 & Down \\
\hline TC21000403.hg.1 & -1.222955 & 0.003335 & Down \\
\hline TC05002143.hg.1 & -1.436058 & 0.003346 & Down \\
\hline TC10000872.hg.1 & -1.210735 & 0.003348 & Down \\
\hline TC02003109.hg.1 & -1.228425 & 0.003361 & Down \\
\hline TC14000289.hg.1 & -1.307652 & 0.003374 & Down \\
\hline TC01002426.hg.1 & -1.214 & 0.003392 & Down \\
\hline TC1 1002900.hg.1 & -1.365245 & 0.0034 & Down \\
\hline TC04000600.hg.1 & -1.241726 & 0.003426 & Down \\
\hline TC02003391.hg.1 & -1.29186 & 0.003429 & Down \\
\hline TC01003999.hg.1 & -1.489871 & 0.003439 & Down \\
\hline TC04001552.hg.1 & -1.2639 & 0.003444 & Down \\
\hline TC12001091.hg.1 & -1.253306 & 0.003463 & Down \\
\hline TC06000930.hg.1 & -1.300405 & 0.003502 & Down \\
\hline TC08000464.hg.1 & -1.271156 & 0.003504 & Down \\
\hline TC05003306.hg.1 & -1.211566 & 0.003515 & Down \\
\hline TC16001224.hg.1 & -1.334827 & 0.003546 & Down \\
\hline TC1 1002505.hg.1 & -1.368315 & 0.003551 & Down \\
\hline TC03000929.hg.1 & -1.21097 & 0.003567 & Down \\
\hline TC15001656.hg.1 & -1.256362 & 0.003592 & Down \\
\hline TC14000427.hg.1 & -1.252462 & 0.003605 & Down \\
\hline TC06003126.hg.1 & -1.282658 & 0.003613 & Down \\
\hline TC08001731.hg.1 & -1.210525 & 0.003616 & Down \\
\hline TCOX000016.hg.1 & -1.222038 & 0.003618 & Down \\
\hline TC10001940.hg.1 & -1.277388 & 0.003624 & Down \\
\hline TC1 1000653.hg.1 & -1.214434 & 0.003626 & Down \\
\hline TC01001760.hg.1 & -1.317357 & 0.003631 & Down \\
\hline TCOX000285.hg.1 & -1.237755 & 0.003634 & Down \\
\hline TC05002272.hg.1 & -1.23028 & 0.003644 & Down \\
\hline TC13000706.hg.1 & -1.295325 & 0.003652 & Down \\
\hline TC07002574.hg.1 & -1.288074 & 0.003655 & Down \\
\hline TC05001287.hg.1 & -1.257783 & 0.003657 & Down \\
\hline TC02002347.hg.1 & -1.216468 & 0.003681 & Down \\
\hline TC19000786.hg.1 & -1.238899 & 0.00372 & Down \\
\hline TC22001164.hg.1 & -1.222475 & 0.003733 & Down \\
\hline TC03000249.hg.1 & -1.322144 & 0.003782 & Down \\
\hline TC02001846.hg.1 & -1.290844 & 0.003798 & Down \\
\hline TC13000062.hg.1 & -1.293684 & 0.003819 & Down \\
\hline TC11000065.hg.1 & -1.20357 & 0.003855 & Down \\
\hline TC01005521.hg.1 & -1.290967 & 0.003878 & Down \\
\hline TC19002432.hg.1 & -1.300036 & 0.003881 & Down \\
\hline TC01005957.hg.1 & -1.320058 & 0.003894 & Down \\
\hline TC03000122.hg.1 & -1.236915 & 0.003915 & Down \\
\hline TC17001999.hg.1 & -1.214198 & 0.003925 & Down \\
\hline TC12002870.hg.1 & -1.233811 & 0.003928 & Down \\
\hline TC01002874.hg.1 & -1.282038 & 0.003933 & Down \\
\hline TC01005965.hg.1 & -1.205432 & 0.003951 & Down \\
\hline TC09002644.hg.1 & -1.242856 & 0.003954 & Down \\
\hline TC05000653.hg.1 & -1.236994 & 0.003956 & Down \\
\hline TC1 1003292.hg.1 & -1.214328 & 0.003964 & Down \\
\hline TC17002315.hg.1 & -1.22301 & 0.00398 & Down \\
\hline TC17000557.hg.1 & -1.378115 & 0.00399 & Down \\
\hline
\end{tabular}

Table 2 (continued)

\begin{tabular}{|c|c|c|c|}
\hline Probe set ID & Fold change & p-value & Gene feature \\
\hline TC11002959.hg.1 & -1.276354 & 0.003993 & Down \\
\hline TC19000244.hg.1 & -1.274288 & 0.003995 & Down \\
\hline TC20000441.hg.1 & -1.2081 & 0.004026 & Down \\
\hline TC16001268.hg.1 & -1.246905 & 0.004029 & Down \\
\hline TC13000651.hg.1 & -1.304621 & 0.004042 & Down \\
\hline TC07003181.hg.1 & -1.236251 & 0.004045 & Down \\
\hline TC01004593.hg.1 & -1.200072 & 0.004047 & Down \\
\hline TC19002136.hg.1 & -1.202992 & 0.004058 & Down \\
\hline TC10000755.hg.1 & -1.320862 & 0.00406 & Down \\
\hline TC02001259.hg.1 & -1.269599 & 0.004097 & Down \\
\hline TC07002494.hg.1 & -1.221869 & 0.004115 & Down \\
\hline TC17001828.hg.1 & -1.355253 & 0.004128 & Down \\
\hline TC12002637.hg.1 & -1.268176 & 0.004133 & Down \\
\hline TC03002947.hg.1 & -1.235059 & 0.004146 & Down \\
\hline TC03003240.hg.1 & -1.243183 & 0.004149 & Down \\
\hline TC10001764.hg.1 & -1.262831 & 0.004211 & Down \\
\hline TC11001522.hg.1 & -1.31939 & 0.004253 & Down \\
\hline TC05002576.hg.1 & -1.280722 & 0.004273 & Down \\
\hline TC11002966.hg.1 & -1.233463 & 0.004304 & Down \\
\hline TCOY000049.hg.1 & -1.326577 & 0.004307 & Down \\
\hline TC14000660.hg.1 & -1.203246 & 0.00431 & Down \\
\hline TC07001989.hg.1 & -1.221607 & 0.004315 & Down \\
\hline TC08001194.hg.1 & -1.343792 & 0.004317 & Down \\
\hline TC18000216.hg.1 & -1.249015 & 0.004346 & Down \\
\hline TC14000282.hg.1 & -1.245247 & 0.004351 & Down \\
\hline TC07003162.hg.1 & -1.203476 & 0.004375 & Down \\
\hline TC08002317.hg.1 & -1.278531 & 0.004377 & Down \\
\hline TC09001614.hg.1 & -1.347077 & 0.00438 & Down \\
\hline TC10002616.hg.1 & -1.303518 & 0.004388 & Down \\
\hline TC11002989.hg.1 & -1.281997 & 0.00439 & Down \\
\hline TC03003014.hg.1 & -1.202168 & 0.004393 & Down \\
\hline TC06003938.hg.1 & -1.266069 & 0.004445 & Down \\
\hline TC03002962.hg.1 & -1.251379 & 0.004481 & Down \\
\hline TC12002295.hg.1 & -1.289958 & 0.004494 & Down \\
\hline $\begin{array}{l}\text { TC6_cox_ } \\
\text { hap2000194.hg.1 }\end{array}$ & -1.306576 & 0.004499 & Down \\
\hline TC15002555.hg.1 & -1.204674 & 0.004502 & Down \\
\hline TC20000160.hg.1 & -1.231493 & 0.00452 & Down \\
\hline TC04002803.hg.1 & -1.318666 & 0.004546 & Down \\
\hline TC04000837.hg.1 & -1.219779 & 0.00459 & Down \\
\hline TC13001207.hg.1 & -1.212648 & 0.004609 & Down \\
\hline TC15000190.hg.1 & -1.209617 & 0.004611 & Down \\
\hline TC02001190.hg.1 & -1.260423 & 0.004629 & Down \\
\hline TC14000251.hg.1 & -1.23846 & 0.004632 & Down \\
\hline TC01002804.hg.1 & -1.237783 & 0.004637 & Down \\
\hline TC16000469.hg.1 & -1.212053 & 0.004666 & Down \\
\hline TC09000173.hg.1 & -1.216822 & 0.004715 & Down \\
\hline TC07002912.hg.1 & -1.268587 & 0.004731 & Down \\
\hline TC19002439.hg.1 & -1.281673 & 0.004759 & Down \\
\hline TC15002362.hg.1 & -1.249402 & 0.004772 & Down \\
\hline TC07001701.hg.1 & -1.251725 & 0.00478 & Down \\
\hline TC06003627.hg.1 & -1.250247 & 0.004783 & Down \\
\hline TC07002791.hg.1 & -1.204493 & 0.004796 & Down \\
\hline
\end{tabular}


Table 2 (continued)

\begin{tabular}{|c|c|c|c|}
\hline Probe set ID & Fold change & p-value & Gene feature \\
\hline TC17001730.hg.1 & -1.228444 & 0.004809 & Down \\
\hline TC03001001.hg.1 & -1.228118 & 0.004897 & Down \\
\hline Probe set ID & Fold change & p-value & Gene feature \\
\hline \multicolumn{4}{|c|}{$\begin{array}{l}\text { (D) Genes up/down-regulated in purified } T C S^{S V 40} \text { compared with non-purified lung } \\
T C s^{S V 40}\end{array}$} \\
\hline TC11000967.hg.1 & -3.281369 & $2.70 E-05$ & Down \\
\hline TC07000780.hg.1 & -1.970289 & $3.00 E-05$ & Down \\
\hline TC11002894.hg.1 & -2.295351 & $3.30 E-05$ & Down \\
\hline TC05000152.hg.1 & -1.620079 & $3.50 E-05$ & Down \\
\hline TC08002510.hg.1 & -1.933489 & $3.80 E-05$ & Down \\
\hline TC03002042.hg.1 & -1.619648 & $4.00 E-05$ & Down \\
\hline TC11001141.hg.1 & -1.537863 & $4.30 E-05$ & Down \\
\hline TC02000336.hg.1 & -1.625021 & 4.60E-05 & Down \\
\hline TC11003109.hg.1 & -2.701391 & $4.80 \mathrm{E}-05$ & Down \\
\hline TC12000188.hg.1 & -1.64374 & $5.10 \mathrm{E}-05$ & Down \\
\hline TC06002006.hg.1 & -1.597063 & $5.30 E-05$ & Down \\
\hline TC08001415.hg.1 & -1.642419 & $5.60 E-05$ & Down \\
\hline TC10000962.hg.1 & -1.45532 & $5.90 \mathrm{E}-05$ & Down \\
\hline TC10000791.hg.1 & -1.651855 & $6.10 E-05$ & Down \\
\hline TC01005437.hg.1 & -1.487989 & $6.40 E-05$ & Down \\
\hline TC19000951.hg.1 & -1.582882 & $6.60 E-05$ & Down \\
\hline TC17002196.hg.1 & 1.361752 & $6.90 E-05$ & Up \\
\hline TC17002479.hg.1 & -1.373139 & $7.70 E-05$ & Down \\
\hline TC02004623.hg.1 & 1.714167 & $7.90 \mathrm{E}-05$ & Up \\
\hline TC16001576.hg.1 & 1.424831 & $8.50 \mathrm{E}-05$ & Up \\
\hline TC06000041.hg.1 & -1.393334 & $8.70 E-05$ & Down \\
\hline TC08002421.hg.1 & 1.950551 & $9.00 \mathrm{E}-05$ & Up \\
\hline TC08002059.hg.1 & -1.709135 & $9.20 \mathrm{E}-05$ & Down \\
\hline TC05001595.hg.1 & 1.323614 & $9.50 \mathrm{E}-05$ & Up \\
\hline TC02004746.hg.1 & 1.393606 & $1.00 \mathrm{E}-04$ & Up \\
\hline TC15002609.hg.1 & 1.312481 & 0.000108 & Up \\
\hline TC16000510.hg.1 & -1.498391 & 0.000111 & Down \\
\hline TC04002890.hg.1 & -1.463155 & 0.000113 & Down \\
\hline TC09002783.hg.1 & -1.50221 & 0.000116 & Down \\
\hline TC14000925.hg.1 & -1.412739 & 0.000124 & Down \\
\hline TC15000651.hg.1 & -1.510536 & 0.000129 & Down \\
\hline TCOM000021.hg.1 & -1.703812 & 0.000131 & Down \\
\hline TC05000861.hg.1 & -1.468789 & 0.000134 & Down \\
\hline TC09000963.hg.1 & -1.357361 & 0.000152 & Down \\
\hline TC22000098.hg.1 & -1.431959 & 0.000163 & Down \\
\hline TC14000667.hg.1 & -1.38192 & 0.000165 & Down \\
\hline TC03002349.hg.1 & -1.491439 & 0.00017 & Down \\
\hline TC03003114.hg.1 & -1.600835 & 0.000183 & Down \\
\hline TC09002305.hg.1 & -1.291981 & 0.000199 & Down \\
\hline TC17000728.hg.1 & -1.727707 & 0.000204 & Down \\
\hline TC04002772.hg.1 & -1.366681 & 0.000207 & Down \\
\hline TC05001371.hg.1 & -1.373092 & 0.000209 & Down \\
\hline TCOX001822.hg.1 & -1.264641 & 0.000212 & Down \\
\hline TC02001623.hg.1 & -1.416467 & 0.000215 & Down \\
\hline TC08001239.hg.1 & -1.365024 & 0.000222 & Down \\
\hline TC03003246.hg.1 & -1.29006 & 0.000228 & Down \\
\hline TC20000203.hg.1 & -1.357278 & 0.000235 & Down \\
\hline
\end{tabular}

Table 2 (continued)

\begin{tabular}{|c|c|c|c|}
\hline Probe set ID & Fold change & p-value & Gene feature \\
\hline TCOM000020.hg.1 & -4.071566 & 0.000259 & Down \\
\hline Probe set ID & Fold change & $p$-value & Gene feature \\
\hline \multicolumn{4}{|c|}{$\begin{array}{l}\text { (E) Genes up/down-regulated in purified TCS }{ }^{S V 40} \text { compared with purified primary lung } \\
\text { TCS }\end{array}$} \\
\hline TC11002382.hg.1 & -4.090002 & $3.30 \mathrm{E}-05$ & Down \\
\hline TC17000728.hg.1 & -3.396128 & $2.70 E-05$ & Down \\
\hline TC11000967.hg.1 & -3.004215 & $9.80 \mathrm{E}-05$ & Down \\
\hline TC08002253.hg.1 & -2.443727 & 0.000155 & Down \\
\hline TC17001801.hg.1 & -2.309379 & $9.00 \mathrm{E}-05$ & Down \\
\hline TC07000780.hg.1 & -2.304878 & $3.80 \mathrm{E}-05$ & Down \\
\hline TC06000639.hg.1 & -2.28677 & $4.60 \mathrm{E}-05$ & Down \\
\hline TC20000045.hg.1 & -2.220467 & 0.00034 & Down \\
\hline TC04001945.hg.1 & -2.095604 & 0.000225 & Down \\
\hline TC12000927.hg.1 & -2.039933 & $4.80 \mathrm{E}-05$ & Down \\
\hline TC13000783.hg.1 & -2.038291 & 0.000288 & Down \\
\hline TC02001799.hg.1 & -2.015351 & 0.002366 & Down \\
\hline TC11002142.hg.1 & -1.996031 & 0.00051 & Down \\
\hline TC16000648.hg.1 & -1.975845 & 0.000783 & Down \\
\hline TC06001978.hg.1 & -1.959951 & $3.00 E-05$ & Down \\
\hline TC09000963.hg.1 & -1.9478 & 0.000152 & Down \\
\hline TC0X001770.hg.1 & -1.947499 & 0.000301 & Down \\
\hline TC09001648.hg.1 & -1.947349 & $6.60 \mathrm{E}-05$ & Down \\
\hline TC04001635.hg.1 & -1.946272 & 0.000205 & Down \\
\hline TC02001940.hg.1 & -1.933961 & $9.50 E-05$ & Down \\
\hline TC15000452.hg.1 & -1.930945 & $4.00 E-05$ & Down \\
\hline TC07000702.hg.1 & -1.910595 & $8.20 E-05$ & Down \\
\hline TCOX001158.hg.1 & -1.88932 & 0.000434 & Down \\
\hline TC08000302.hg.1 & -1.854536 & $5.10 \mathrm{E}-05$ & Down \\
\hline TC20000952.hg.1 & -1.846904 & 0.00015 & Down \\
\hline TCOX000985.hg.1 & -1.821657 & 0.000168 & Down \\
\hline TC07001784.hg.1 & -1.818055 & $4.30 E-05$ & Down \\
\hline TC09000971.hg.1 & -1.814467 & $8.50 E-05$ & Down \\
\hline TC08001415.hg.1 & -1.813106 & $6.40 E-05$ & Down \\
\hline TC09000096.hg.1 & -1.808112 & 0.000392 & Down \\
\hline TC02000205.hg.1 & -1.798757 & 0.00016 & Down \\
\hline TC12000188.hg.1 & -1.798296 & $7.40 \mathrm{E}-05$ & Down \\
\hline TC01003308.hg.1 & -1.786611 & 0.000171 & Down \\
\hline TC19000767.hg.1 & -1.780988 & $1.00 \mathrm{E}-04$ & Down \\
\hline TC05000152.hg.1 & -1.780756 & 0.000121 & Down \\
\hline TC02000336.hg.1 & -1.777235 & $8.70 E-05$ & Down \\
\hline TC02002456.hg.1 & -1.775443 & $8.00 E-05$ & Down \\
\hline TC02000396.hg.1 & -1.770308 & $5.30 \mathrm{E}-05$ & Down \\
\hline TC12000633.hg.1 & -1.765148 & 7.70E-05 & Down \\
\hline TC05001593.hg.1 & -1.752631 & $7.20 \mathrm{E}-05$ & Down \\
\hline TC19000951.hg.1 & -1.750403 & 0.000124 & Down \\
\hline TC09001461.hg.1 & -1.742247 & 0.000111 & Down \\
\hline TC03002042.hg.1 & -1.741147 & 0.000103 & Down \\
\hline TC12001286.hg.1 & -1.726658 & 0.000176 & Down \\
\hline TC06002006.hg.1 & -1.707544 & 0.000132 & Down \\
\hline TC01006068.hg.1 & -1.692979 & 0.000134 & Down \\
\hline TC05002800.hg.1 & -1.691325 & 0.000312 & Down \\
\hline TC16000510.hg.1 & -1.682589 & 0.00027 & Down \\
\hline
\end{tabular}


Table 2 (continued)

\begin{tabular}{|c|c|c|c|}
\hline Probe set ID & Fold change & $p$-value & Gene feature \\
\hline TC01002594.hg.1 & -1.674638 & 0.000486 & Down \\
\hline TC16001289.hg.1 & -1.671928 & $5.90 \mathrm{E}-05$ & Down \\
\hline TC10000791.hg.1 & -1.658964 & 0.000272 & Down \\
\hline TC01002663.hg.1 & -1.6563 & 0.002108 & Down \\
\hline TC12002688.hg.1 & -1.654776 & 0.000166 & Down \\
\hline TC20000650.hg.1 & -1.641248 & 0.000126 & Down \\
\hline TC11001010.hg.1 & -1.634925 & 0.000192 & Down \\
\hline TC02004814.hg.1 & -1.627547 & 0.000257 & Down \\
\hline TC03000874.hg.1 & -1.624355 & 0.00168 & Down \\
\hline TC20000373.hg.1 & -1.622438 & 0.000106 & Down \\
\hline TCOX001624.hg.1 & -1.620312 & 0.000523 & Down \\
\hline TC22000098.hg.1 & -1.615575 & 0.000246 & Down \\
\hline TC01002798.hg.1 & -1.61537 & 0.000262 & Down \\
\hline TCOX001292.hg.1 & -1.607909 & 0.001472 & Down \\
\hline TC09002651.hg.1 & -1.603388 & 0.003161 & Down \\
\hline TC05000708.hg.1 & -1.59612 & 0.000254 & Down \\
\hline TC12000244.hg.1 & -1.589748 & 0.000817 & Down \\
\hline TC01002746.hg.1 & -1.585136 & 0.000377 & Down \\
\hline TCOX001411.hg.1 & -1.576617 & 0.000484 & Down \\
\hline TC01002633.hg.1 & -1.57574 & 0.000447 & Down \\
\hline TC14000925.hg.1 & -1.5746 & 0.000332 & Down \\
\hline TC06004042.hg.1 & -1.572429 & 0.000173 & Down \\
\hline TC03001841.hg.1 & -1.571987 & 0.000129 & Down \\
\hline TC02001302.hg.1 & -1.571147 & 0.000223 & Down \\
\hline TC09001534.hg.1 & -1.570612 & 0.000457 & Down \\
\hline TC20001199.hg.1 & -1.567384 & 0.000431 & Down \\
\hline TC02001432.hg.1 & -1.56216 & 0.000306 & Down \\
\hline TC04000508.hg.1 & -1.560484 & 0.000202 & Down \\
\hline TC06001365.hg.1 & -1.559188 & 0.000689 & Down \\
\hline TC11002077.hg.1 & -1.558571 & 0.000489 & Down \\
\hline TC02002994.hg.1 & -1.554748 & 0.000744 & Down \\
\hline TC15001073.hg.1 & -1.553387 & 0.000645 & Down \\
\hline TC02001602.hg.1 & -1.549962 & 0.000218 & Down \\
\hline TCOX001618.hg.1 & -1.549945 & 0.000405 & Down \\
\hline TC15002586.hg.1 & -1.54835 & 0.000593 & Down \\
\hline TC22001417.hg.1 & -1.548268 & 0.000382 & Down \\
\hline TC05000861.hg.1 & -1.545398 & 0.00045 & Down \\
\hline TC19000163.hg.1 & -1.544743 & 0.000145 & Down \\
\hline TC06000568.hg.1 & -1.542824 & 0.000343 & Down \\
\hline TC08000801.hg.1 & -1.540416 & 0.000137 & Down \\
\hline TC08002335.hg.1 & -1.538863 & 0.003182 & Down \\
\hline TC1 1002486.hg.1 & -1.538725 & 0.000293 & Down \\
\hline TCOX000279.hg.1 & -1.534468 & 0.002392 & Down \\
\hline TC10000866.hg.1 & -1.528931 & 0.000252 & Down \\
\hline TC10000962.hg.1 & -1.528853 & 0.000184 & Down \\
\hline TC15000651.hg.1 & -1.527445 & 0.0024 & Down \\
\hline TC09001602.hg.1 & -1.527117 & 0.000285 & Down \\
\hline TC14000052.hg.1 & -1.523268 & 0.000666 & Down \\
\hline TC05000621.hg.1 & -1.521581 & 0.000945 & Down \\
\hline TC03000195.hg.1 & -1.520243 & 0.000239 & Down \\
\hline TC17001773.hg.1 & -1.514647 & 0.000199 & Down \\
\hline TC02001366.hg.1 & -1.514152 & 0.00033 & Down \\
\hline
\end{tabular}

Table 2 (continued)

\begin{tabular}{|c|c|c|c|}
\hline Probe set ID & Fold change & $p$-value & Gene feature \\
\hline TC08001315.hg.1 & -1.513587 & 0.000669 & Down \\
\hline TC11002476.hg.1 & -1.512413 & 0.000549 & Down \\
\hline TC15001473.hg.1 & -1.511023 & 0.000439 & Down \\
\hline TC07001655.hg.1 & -1.508092 & 0.000624 & Down \\
\hline TC09000100.hg.1 & -1.506552 & 0.001224 & Down \\
\hline TC05002818.hg.1 & -1.505908 & 0.000374 & Down \\
\hline TC11001141.hg.1 & -1.505044 & 0.000882 & Down \\
\hline TC0X001410.hg.1 & -1.504643 & 0.000497 & Down \\
\hline TC22001175.hg.1 & -1.504265 & 0.000418 & Down \\
\hline TC08000450.hg.1 & -1.497675 & 0.000442 & Down \\
\hline TC17000491.hg.1 & -1.494822 & 0.000478 & Down \\
\hline TC19001173.hg.1 & -1.48853 & 0.003494 & Down \\
\hline TC15001326.hg.1 & -1.486591 & 0.000113 & Down \\
\hline TC16002013.hg.1 & -1.486347 & 0.000228 & Down \\
\hline TC15000631.hg.1 & -1.485978 & 0.000356 & Down \\
\hline TC12001052.hg.1 & -1.485625 & 0.001544 & Down \\
\hline TC16000853.hg.1 & -1.48534 & 0.001049 & Down \\
\hline TC01002976.hg.1 & -1.483998 & 0.000244 & Down \\
\hline TC12001449.hg.1 & -1.481302 & 0.000358 & Down \\
\hline TC12001995.hg.1 & -1.476015 & 0.000504 & Down \\
\hline TC0X001136.hg.1 & -1.473457 & 0.000265 & Down \\
\hline TC06001064.hg.1 & -1.473031 & 0.001156 & Down \\
\hline TC09000516.hg.1 & -1.466985 & 0.001977 & Down \\
\hline TC09001936.hg.1 & -1.461819 & 0.001954 & Down \\
\hline TC13001206.hg.1 & -1.461709 & 0.002804 & Down \\
\hline TC14000620.hg.1 & -1.461092 & 0.000197 & Down \\
\hline TC10002439.hg.1 & -1.460412 & 0.001052 & Down \\
\hline TC07000139.hg.1 & -1.459424 & 0.000979 & Down \\
\hline TC04002443.hg.1 & -1.457362 & 0.00063 & Down \\
\hline TCOX000450.hg.1 & -1.456236 & 0.00052 & Down \\
\hline TC03001571.hg.1 & -1.454824 & 0.000327 & Down \\
\hline TC06002801.hg.1 & -1.452915 & 0.000283 & Down \\
\hline TC10002919.hg.1 & -1.452582 & 0.000259 & Down \\
\hline TC02000955.hg.1 & -1.450519 & 0.001299 & Down \\
\hline TC05002279.hg.1 & -1.449507 & 0.000416 & Down \\
\hline TC09002783.hg.1 & -1.445945 & 0.000452 & Down \\
\hline TC01003510.hg.1 & -1.445715 & 0.000364 & Down \\
\hline TC0X001762.hg.1 & -1.445315 & 0.000554 & Down \\
\hline TC22000400.hg.1 & -1.445268 & 0.000296 & Down \\
\hline TC02001623.hg.1 & -1.444939 & 0.00089 & Down \\
\hline TC07001706.hg.1 & -1.442104 & 0.001574 & Down \\
\hline TC07001709.hg.1 & -1.442104 & 0.001574 & Down \\
\hline TC08000652.hg.1 & -1.441877 & 0.001378 & Down \\
\hline TC15001604.hg.1 & -1.440453 & 0.000463 & Down \\
\hline TC04001524.hg.1 & -1.437658 & 0.000158 & Down \\
\hline TC14001281.hg.1 & -1.433784 & 0.000471 & Down \\
\hline TC02002768.hg.1 & -1.428308 & 0.00059 & Down \\
\hline TC05002975.hg.1 & -1.427776 & 0.000421 & Down \\
\hline TC10001432.hg.1 & -1.427505 & 7.00E -04 & Down \\
\hline TC06000041.hg.1 & -1.427026 & 0.000319 & Down \\
\hline TC06000705.hg.1 & -1.426715 & 0.000984 & Down \\
\hline TC01002620.hg.1 & -1.426413 & 0.00039 & Down \\
\hline
\end{tabular}


Table 2 (continued)

\begin{tabular}{|c|c|c|c|}
\hline Probe set ID & Fold change & $p$-value & Gene feature \\
\hline TC06003710.hg.1 & -1.420694 & 0.003033 & Down \\
\hline TC07000339.hg.1 & -1.419104 & 0.00094 & Down \\
\hline TC05000526.hg.1 & -1.417126 & 0.000345 & Down \\
\hline TC02001836.hg.1 & -1.417083 & 0.000971 & Down \\
\hline TC01003990.hg.1 & -1.4165 & 0.001091 & Down \\
\hline TC10002673.hg.1 & -1.416168 & 0.001312 & Down \\
\hline TC21000483.hg.1 & -1.415996 & 0.002217 & Down \\
\hline TC02003914.hg.1 & -1.413985 & 0.000627 & Down \\
\hline TC07001267.hg.1 & -1.412486 & 0.000437 & Down \\
\hline TC10002723.hg.1 & -1.411693 & 0.002092 & Down \\
\hline TC03000155.hg.1 & -1.410721 & 0.001591 & Down \\
\hline TC10000793.hg.1 & -1.409945 & 0.001221 & Down \\
\hline TC03001056.hg.1 & -1.408329 & 0.000278 & Down \\
\hline TC10000686.hg.1 & -1.407419 & 0.000215 & Down \\
\hline TC14001892.hg.1 & -1.406093 & 0.000366 & Down \\
\hline TC21000698.hg.1 & -1.405176 & 0.0012 & Down \\
\hline TC06001651.hg.1 & -1.404117 & 0.000139 & Down \\
\hline TC22000272.hg.1 & -1.402122 & 0.002227 & Down \\
\hline TC14002171.hg.1 & -1.401997 & 0.000528 & Down \\
\hline TC06002303.hg.1 & -1.401899 & 0.001466 & Down \\
\hline TC10001189.hg.1 & -1.400004 & 0.000611 & Down \\
\hline TC18000381.hg.1 & -1.398874 & 0.001571 & Down \\
\hline TC01002339.hg.1 & -1.398183 & 0.000408 & Down \\
\hline TC14001090.hg.1 & -1.396639 & 0.001443 & Down \\
\hline TC02002028.hg.1 & -1.39541 & 0.001099 & Down \\
\hline TC07001289.hg.1 & -1.395341 & 0.001604 & Down \\
\hline TC07000690.hg.1 & -1.394396 & 0.000317 & Down \\
\hline TC11001479.hg.1 & -1.394041 & 0.001657 & Down \\
\hline TC12003094.hg.1 & -1.391962 & 0.001722 & Down \\
\hline TC03000823.hg.1 & -1.390073 & 0.000619 & Down \\
\hline TC05002924.hg.1 & -1.388742 & 0.001779 & Down \\
\hline TC05000703.hg.1 & -1.388715 & 0.00369 & Down \\
\hline TC12000231.hg.1 & -1.388427 & 0.000186 & Down \\
\hline TC03002201.hg.1 & -1.38807 & 0.001104 & Down \\
\hline TC05000688.hg.1 & -1.387472 & 0.000776 & Down \\
\hline TC09001410.hg.1 & -1.385276 & 0.002814 & Down \\
\hline TC01003999.hg.1 & -1.384791 & 0.001714 & Down \\
\hline TC03000962.hg.1 & -1.384037 & 0.002342 & Down \\
\hline TC10001912.hg.1 & -1.381688 & 0.0015 & Down \\
\hline TC01002393.hg.1 & -1.381656 & 0.00193 & Down \\
\hline TC05000214.hg.1 & -1.378498 & 0.001206 & Down \\
\hline TC02001248.hg.1 & -1.377049 & 0.000515 & Down \\
\hline TCOX002258.hg.1 & -1.375302 & 0.000833 & Down \\
\hline TC16002030.hg.1 & -1.374782 & 0.000395 & Down \\
\hline TC03000629.hg.1 & -1.37476 & 0.001255 & Down \\
\hline TC07001657.hg.1 & -1.373948 & 0.001018 & Down \\
\hline TCOX000283.hg.1 & -1.373835 & 0.003038 & Down \\
\hline TC10000755.hg.1 & -1.372467 & 0.003283 & Down \\
\hline TC06000824.hg.1 & -1.371586 & 0.002728 & Down \\
\hline TC11000089.hg.1 & -1.371419 & 0.000371 & Down \\
\hline TC18000050.hg.1 & -1.370569 & 0.001226 & Down \\
\hline TC16001555.hg.1 & -1.369024 & 0.000387 & Down \\
\hline
\end{tabular}

Table 2 (continued)

\begin{tabular}{|c|c|c|c|}
\hline Probe set ID & Fold change & $p$-value & Gene feature \\
\hline TC20000340.hg.1 & -1.368516 & 0.00363 & Down \\
\hline TC01005517.hg.1 & -1.368066 & 0.002598 & Down \\
\hline TCOX000232.hg.1 & -1.36715 & 0.001594 & Down \\
\hline TC01004166.hg.1 & -1.365917 & 0.000976 & Down \\
\hline TC08001640.hg.1 & -1.365564 & 0.002254 & Down \\
\hline TC05000183.hg.1 & -1.365261 & 0.001818 & Down \\
\hline TC05000876.hg.1 & -1.364983 & 0.000961 & Down \\
\hline TC10000439.hg.1 & -1.364697 & 0.000298 & Down \\
\hline TC09002445.hg.1 & -1.364017 & 0.002141 & Down \\
\hline TC03002972.hg.1 & -1.36302 & 0.001763 & Down \\
\hline TC09001709.hg.1 & -1.362426 & 0.00119 & Down \\
\hline TC09002851.hg.1 & -1.36068 & 0.000577 & Down \\
\hline TC21000931.hg.1 & -1.360378 & 0.000596 & Down \\
\hline TC05002802.hg.1 & -1.360064 & 0.003648 & Down \\
\hline TC05002034.hg.1 & -1.358914 & 0.001698 & Down \\
\hline TC11000768.hg.1 & -1.358104 & 0.001164 & Down \\
\hline TC11000808.hg.1 & -1.357692 & 4.00E-04 & Down \\
\hline TC05002959.hg.1 & -1.356675 & 0.000739 & Down \\
\hline TC16001991.hg.1 & -1.355237 & 0.000426 & Down \\
\hline TC03001321.hg.1 & -1.352844 & 0.000476 & Down \\
\hline TC17000230.hg.1 & -1.352713 & 0.000935 & Down \\
\hline TC05001562.hg.1 & -1.352471 & 0.002853 & Down \\
\hline TC06002451.hg.1 & -1.351413 & 0.003281 & Down \\
\hline TC14000693.hg.1 & -1.351022 & 0.001693 & Down \\
\hline TC06001125.hg.1 & -1.350964 & 0.003685 & Down \\
\hline TC12000805.hg.1 & -1.349635 & 0.001664 & Down \\
\hline TC10000815.hg.1 & -1.349597 & 0.000682 & Down \\
\hline TC08000752.hg.1 & -1.348369 & 0.003401 & Down \\
\hline TC01005988.hg.1 & -1.347966 & 0.001354 & Down \\
\hline TC16001082.hg.1 & -1.347545 & 0.001967 & Down \\
\hline TC06000972.hg.1 & -1.347335 & 0.001701 & Down \\
\hline TC08001540.hg.1 & -1.345966 & 0.001112 & Down \\
\hline TC04000854.hg.1 & -1.345957 & 0.000384 & Down \\
\hline TC10001536.hg.1 & -1.345921 & 0.00352 & Down \\
\hline TC05003369.hg.1 & -1.345436 & 0.000575 & Down \\
\hline TC16000560.hg.1 & -1.344377 & 0.001315 & Down \\
\hline TC01006043.hg.1 & -1.344027 & 0.000786 & Down \\
\hline TC04000811.hg.1 & -1.343967 & 0.000304 & Down \\
\hline TC05000998.hg.1 & -1.343336 & 0.002569 & Down \\
\hline TC19000170.hg.1 & -1.342442 & 0.003635 & Down \\
\hline TC17000742.hg.1 & -1.340971 & 0.002566 & Down \\
\hline TC21000902.hg.1 & -1.340061 & 0.00187 & Down \\
\hline TC15002522.hg.1 & -1.339697 & 0.000781 & Down \\
\hline TC12000736.hg.1 & -1.339218 & 0.003919 & Down \\
\hline TC12003194.hg.1 & -1.339102 & 0.000544 & Down \\
\hline TC11002900.hg.1 & -1.338332 & 0.001826 & Down \\
\hline TC04001241.hg.1 & -1.338326 & 0.000875 & Down \\
\hline TC10001843.hg.1 & -1.337933 & 0.000309 & Down \\
\hline TC02002711.hg.1 & -1.337676 & 0.000491 & Down \\
\hline TC07001656.hg.1 & -1.336388 & 0.001578 & Down \\
\hline TC0X000986.hg.1 & -1.336275 & 0.001122 & Down \\
\hline TC19000349.hg.1 & -1.336181 & 0.00149 & Down \\
\hline
\end{tabular}


Table 2 (continued)

\begin{tabular}{|c|c|c|c|}
\hline Probe set ID & Fold change & $p$-value & Gene feature \\
\hline TC12000238.hg.1 & -1.334203 & 0.000291 & Down \\
\hline TC09001898.hg.1 & -1.333912 & 0.001047 & Down \\
\hline TC02001429.hg.1 & -1.332234 & 0.000635 & Down \\
\hline TC10000827.hg.1 & -1.332045 & 0.002267 & Down \\
\hline TC13001120.hg.1 & -1.330949 & 0.001847 & Down \\
\hline TC01006051.hg.1 & -1.330512 & 0.002522 & Down \\
\hline TC05002063.hg.1 & -1.330054 & 0.000411 & Down \\
\hline TC13000501.hg.1 & -1.329266 & 0.001526 & Down \\
\hline TC03001672.hg.1 & -1.328838 & 0.000468 & Down \\
\hline TC05000595.hg.1 & -1.326941 & 0.002921 & Down \\
\hline TC0X000282.hg.1 & -1.326422 & 0.000749 & Down \\
\hline TC16000673.hg.1 & -1.326046 & 0.001391 & Down \\
\hline TC20000841.hg.1 & -1.325451 & 0.001234 & Down \\
\hline TC21000149.hg.1 & -1.32514 & 0.002991 & Down \\
\hline TC14001177.hg.1 & -1.324741 & 0.002066 & Down \\
\hline TC03000054.hg.1 & -1.324079 & 0.004008 & Down \\
\hline TC0X001094.hg.1 & -1.323445 & 0.003145 & Down \\
\hline TC08001239.hg.1 & -1.323048 & 0.003476 & Down \\
\hline TC01005264.hg.1 & -1.322568 & 0.000893 & Down \\
\hline TC17002295.hg.1 & -1.322392 & 0.002183 & Down \\
\hline TC09000452.hg.1 & -1.322086 & 0.001067 & Down \\
\hline TC08002500.hg.1 & -1.32044 & 0.000236 & Down \\
\hline TC10002210.hg.1 & -1.316607 & 0.00393 & Down \\
\hline TC03000845.hg.1 & -1.315832 & 0.00375 & Down \\
\hline TC06003126.hg.1 & -1.315786 & 0.00058 & Down \\
\hline TC05000611.hg.1 & -1.31565 & 0.001065 & Down \\
\hline TCOX000127.hg.1 & -1.314996 & 0.00143 & Down \\
\hline TC16001996.hg.1 & -1.314795 & 0.00175 & Down \\
\hline TC08000285.hg.1 & -1.314727 & 0.001912 & Down \\
\hline TC07002742.hg.1 & -1.312996 & 0.000481 & Down \\
\hline TC02000971.hg.1 & -1.311939 & 0.00259 & Down \\
\hline TCOX001939.hg.1 & -1.311233 & 0.001636 & Down \\
\hline TC10001198.hg.1 & -1.310689 & 0.001399 & Down \\
\hline TC06001254.hg.1 & -1.310172 & 0.002178 & Down \\
\hline TC17000164.hg.1 & -1.310162 & 0.000987 & Down \\
\hline TC01000642.hg.1 & -1.30967 & 0.001005 & Down \\
\hline TC19000785.hg.1 & -1.309054 & 0.000687 & Down \\
\hline TC07000478.hg.1 & -1.308947 & 0.002363 & Down \\
\hline TC09001635.hg.1 & -1.307954 & 0.000718 & Down \\
\hline TCOX001901.hg.1 & -1.305637 & 0.000525 & Down \\
\hline TC08001005.hg.1 & -1.305589 & 0.003865 & Down \\
\hline TC07002764.hg.1 & -1.305001 & 0.001617 & Down \\
\hline TC01003317.hg.1 & -1.304001 & 0.000734 & Down \\
\hline TC14002114.hg.1 & -1.303302 & 0.001654 & Down \\
\hline TC05001142.hg.1 & -1.30304 & 0.001915 & Down \\
\hline TC20000564.hg.1 & -1.302837 & 0.000908 & Down \\
\hline TC01005249.hg.1 & -1.30211 & 0.000911 & Down \\
\hline TC09002102.hg.1 & -1.301834 & 0.003586 & Down \\
\hline TC10002728.hg.1 & -1.301751 & 0.001511 & Down \\
\hline TC14000711.hg.1 & -1.301227 & 0.001088 & Down \\
\hline TC09000942.hg.1 & -1.299524 & 0.001607 & Down \\
\hline TC22000010.hg.1 & -1.296747 & 0.00174 & Down \\
\hline
\end{tabular}

Table 2 (continued)

\begin{tabular}{|c|c|c|c|}
\hline Probe set ID & Fold change & $p$-value & Gene feature \\
\hline TC03002116.hg.1 & -1.296625 & 0.000773 & Down \\
\hline TC01000843.hg.1 & -1.294777 & 0.001863 & Down \\
\hline TC12001919.hg.1 & -1.292031 & 0.002817 & Down \\
\hline TC05001371.hg.1 & -1.291558 & 0.001479 & Down \\
\hline TC07001870.hg.1 & -1.291055 & 0.000901 & Down \\
\hline TC01000565.hg.1 & -1.290245 & 0.000695 & Down \\
\hline TC12002368.hg.1 & -1.290229 & 0.000606 & Down \\
\hline TC07000399.hg.1 & -1.28954 & 0.003703 & Down \\
\hline TC19000423.hg.1 & -1.288189 & 0.002329 & Down \\
\hline TC07002506.hg.1 & -1.28737 & 0.000997 & Down \\
\hline TC02000092.hg.1 & -1.286776 & 0.000632 & Down \\
\hline TC13001372.hg.1 & -1.286649 & 0.001357 & Down \\
\hline TC03003014.hg.1 & -1.286006 & 0.003111 & Down \\
\hline TC12001455.hg.1 & -1.28555 & 0.003453 & Down \\
\hline TCOX000233.hg.1 & -1.283645 & 0.002621 & Down \\
\hline TC19000409.hg.1 & -1.281773 & 0.001943 & Down \\
\hline TC11000172.hg.1 & -1.280685 & 0.003312 & Down \\
\hline TC07002026.hg.1 & -1.280371 & 0.00071 & Down \\
\hline TC07002763.hg.1 & -1.279866 & 0.000752 & Down \\
\hline TC07002428.hg.1 & -1.279296 & 0.001135 & Down \\
\hline TC01002397.hg.1 & -1.278713 & 0.000921 & Down \\
\hline TC13000269.hg.1 & -1.277383 & 0.002016 & Down \\
\hline TC20000763.hg.1 & -1.276423 & 0.003633 & Down \\
\hline TC11000705.hg.1 & -1.276067 & 0.000507 & Down \\
\hline TC06003416.hg.1 & -1.275874 & 0.000713 & Down \\
\hline TC03000687.hg.1 & -1.275833 & 0.00199 & Down \\
\hline TC12001821.hg.1 & -1.275762 & 0.003067 & Down \\
\hline TC01006074.hg.1 & -1.275058 & 0.002324 & Down \\
\hline TC07000389.hg.1 & -1.274792 & 0.002663 & Down \\
\hline TC03000673.hg.1 & -1.272024 & 0.002235 & Down \\
\hline TC10001951.hg.1 & -1.271122 & 0.000848 & Down \\
\hline TC17001193.hg.1 & -1.270112 & 0.000804 & Down \\
\hline TC08002312.hg.1 & -1.269922 & 0.001349 & Down \\
\hline TC04002663.hg.1 & -1.269488 & 0.002032 & Down \\
\hline TC01004768.hg.1 & -1.269265 & 0.001148 & Down \\
\hline TC01005463.hg.1 & -1.268925 & 0.002908 & Down \\
\hline TC16001699.hg.1 & -1.265388 & 0.00057 & Down \\
\hline TC01005508.hg.1 & -1.265174 & 0.002194 & Down \\
\hline TC21000491.hg.1 & -1.265062 & 0.00162 & Down \\
\hline TC03002000.hg.1 & -1.264798 & 0.001555 & Down \\
\hline TC21000706.hg.1 & -1.264128 & 0.003956 & Down \\
\hline TC15000396.hg.1 & -1.263935 & 0.001297 & Down \\
\hline TC20000800.hg.1 & -1.263339 & 0.001276 & Down \\
\hline TC07002757.hg.1 & -1.263139 & 0.001002 & Down \\
\hline TC03001494.hg.1 & -1.263001 & 0.003127 & Down \\
\hline TC10001532.hg.1 & -1.262834 & 0.002603 & Down \\
\hline TC19000162.hg.1 & -1.262821 & 0.002081 & Down \\
\hline TC15001184.hg.1 & -1.262007 & 0.000835 & Down \\
\hline TC15001480.hg.1 & -1.261402 & 0.001975 & Down \\
\hline TC02001762.hg.1 & -1.259495 & 0.001305 & Down \\
\hline TC08000460.hg.1 & -1.259301 & 0.003218 & Down \\
\hline TC08002256.hg.1 & -1.258796 & 0.00065 & Down \\
\hline
\end{tabular}


Table 2 (continued)

\begin{tabular}{|c|c|c|c|}
\hline Probe set ID & Fold change & $p$-value & Gene feature \\
\hline TC15001294.hg.1 & -1.257698 & 0.002676 & Down \\
\hline TC12000589.hg.1 & -1.257295 & 0.001195 & Down \\
\hline TC03000342.hg.1 & -1.257262 & 0.004063 & Down \\
\hline TC07002787.hg.1 & -1.25713 & 0.002561 & Down \\
\hline TC21000925.hg.1 & -1.257035 & 0.000974 & Down \\
\hline TC03003257.hg.1 & -1.256746 & 0.000583 & Down \\
\hline TC07002052.hg.1 & -1.256682 & 0.001404 & Down \\
\hline TC07002196.hg.1 & -1.256575 & 0.001383 & Down \\
\hline TC17002053.hg.1 & -1.256349 & 0.001417 & Down \\
\hline TC09000159.hg.1 & -1.255888 & 0.001346 & Down \\
\hline TC05001001.hg.1 & -1.25565 & 0.000989 & Down \\
\hline TC05002401.hg.1 & -1.25502 & 0.001151 & Down \\
\hline TC0X002335.hg.1 & -1.254604 & 0.002892 & Down \\
\hline TC21000447.hg.1 & -1.25417 & 0.001539 & Down \\
\hline TC12001854.hg.1 & -1.253701 & 0.00161 & Down \\
\hline TC02002409.hg.1 & -1.252127 & 0.00077 & Down \\
\hline TC09002847.hg.1 & -1.251779 & 0.001474 & Down \\
\hline TC12001777.hg.1 & -1.250828 & 0.002212 & Down \\
\hline TC20000261.hg.1 & -1.25079 & 0.002373 & Down \\
\hline TC12000184.hg.1 & -1.249185 & 0.002347 & Down \\
\hline TC05002180.hg.1 & -1.247857 & 0.00076 & Down \\
\hline TC08001323.hg.1 & -1.246702 & 0.00308 & Down \\
\hline TC05002576.hg.1 & -1.246539 & 0.000807 & Down \\
\hline TC11002740.hg.1 & -1.245999 & 0.004083 & Down \\
\hline TC04001485.hg.1 & -1.245869 & 0.00345 & Down \\
\hline TC14001767.hg.1 & -1.245539 & 0.001281 & Down \\
\hline TC02001858.hg.1 & -1.244836 & 0.001487 & Down \\
\hline TC02004683.hg.1 & -1.244802 & 0.001341 & Down \\
\hline TC01000383.hg.1 & -1.244648 & 0.001641 & Down \\
\hline TC03001231.hg.1 & -1.244214 & 0.001031 & Down \\
\hline TC05002808.hg.1 & -1.24399 & 0.00222 & Down \\
\hline TC02003539.hg.1 & -1.243551 & 0.001615 & Down \\
\hline TC15001493.hg.1 & -1.243527 & 0.001026 & Down \\
\hline TC16000769.hg.1 & -1.242087 & 0.003841 & Down \\
\hline TC07003221.hg.1 & -1.241589 & 0.00302 & Down \\
\hline TC18000715.hg.1 & -1.241272 & 0.001013 & Down \\
\hline TC17001238.hg.1 & -1.24064 & 0.001964 & Down \\
\hline TC20000751.hg.1 & -1.24047 & 0.002572 & Down \\
\hline TC17001774.hg.1 & -1.240464 & 0.001138 & Down \\
\hline TC01004651.hg.1 & -1.239979 & 0.003361 & Down \\
\hline TC08000280.hg.1 & -1.239825 & 0.004026 & Down \\
\hline TC01003671.hg.1 & -1.239653 & 0.001271 & Down \\
\hline TC06001540.hg.1 & -1.239545 & 0.003541 & Down \\
\hline TC1 1002993.hg.1 & -1.239126 & 0.003369 & Down \\
\hline TC08002317.hg.1 & -1.238759 & 0.000948 & Down \\
\hline TC12003089.hg.1 & -1.23843 & 0.000906 & Down \\
\hline TC12001102.hg.1 & -1.237826 & 0.002626 & Down \\
\hline TC1 1002750.hg.1 & -1.237261 & 0.001482 & Down \\
\hline TC12002592.hg.1 & -1.236436 & 0.003643 & Down \\
\hline TC1 1001275.hg.1 & -1.235994 & 0.003359 & Down \\
\hline TC08001580.hg.1 & -1.235099 & 0.001273 & Down \\
\hline TC17001239.hg.1 & -1.234648 & 0.00229 & Down \\
\hline TCOY000302.hg.1 & -1.234355 & 0.003678 & Down \\
\hline
\end{tabular}

Table 2 (continued)

\begin{tabular}{|c|c|c|c|}
\hline Probe set ID & Fold change & $p$-value & Gene feature \\
\hline TCOY000304.hg.1 & -1.234355 & 0.003678 & Down \\
\hline TC22000194.hg.1 & -1.23392 & 0.003023 & Down \\
\hline TC02001892.hg.1 & -1.233697 & 0.002035 & Down \\
\hline TC07001419.hg.1 & -1.233028 & 0.003205 & Down \\
\hline TC02004514.hg.1 & -1.232891 & 0.001096 & Down \\
\hline TC11000365.hg.1 & -1.232652 & 0.003434 & Down \\
\hline TC17001310.hg.1 & -1.232628 & 0.001917 & Down \\
\hline TC04000223.hg.1 & -1.232273 & 0.002785 & Down \\
\hline TC02003541.hg.1 & -1.231717 & 0.002243 & Down \\
\hline TC10002633.hg.1 & -1.231394 & 0.004024 & Down \\
\hline TC14002045.hg.1 & -1.231387 & 0.003135 & Down \\
\hline TC14000688.hg.1 & -1.231155 & 0.001599 & Down \\
\hline TC10000133.hg.1 & -1.231146 & 0.003936 & Down \\
\hline TC10000136.hg.1 & -1.231146 & 0.003936 & Down \\
\hline TC01003920.hg.1 & -1.230188 & 0.002484 & Down \\
\hline TC01003921.hg.1 & -1.230188 & 0.002484 & Down \\
\hline TC01003922.hg.1 & -1.230188 & 0.002484 & Down \\
\hline TC01003923.hg.1 & -1.230188 & 0.002484 & Down \\
\hline TC01003924.hg.1 & -1.230188 & 0.002484 & Down \\
\hline TC01003925.hg.1 & -1.230188 & 0.002484 & Down \\
\hline TC01003926.hg.1 & -1.230188 & 0.002484 & Down \\
\hline TC01003927.hg.1 & -1.230188 & 0.002484 & Down \\
\hline TC01003929.hg.1 & -1.230188 & 0.002484 & Down \\
\hline TC01003930.hg.1 & -1.230188 & 0.002484 & Down \\
\hline TC01003931.hg.1 & -1.230188 & 0.002484 & Down \\
\hline TC01003932.hg.1 & -1.230188 & 0.002484 & Down \\
\hline TC01003933.hg.1 & -1.230188 & 0.002484 & Down \\
\hline TC01003934.hg.1 & -1.230188 & 0.002484 & Down \\
\hline TC01003935.hg.1 & -1.230188 & 0.002484 & Down \\
\hline TC01003936.hg.1 & -1.230188 & 0.002484 & Down \\
\hline TC11000769.hg.1 & -1.229189 & 0.002433 & Down \\
\hline TC01004020.hg.1 & -1.22836 & 0.002014 & Down \\
\hline TC22001053.hg.1 & -1.22805 & 0.003752 & Down \\
\hline TC04001733.hg.1 & -1.227708 & 0.001127 & Down \\
\hline TC0X000808.hg.1 & -1.22752 & 0.001289 & Down \\
\hline TC01004433.hg.1 & -1.226181 & 0.004164 & Down \\
\hline TC21000029.hg.1 & -1.225461 & 0.001704 & Down \\
\hline TC03002704.hg.1 & -1.224861 & 0.001445 & Down \\
\hline TC02004897.hg.1 & -1.224747 & 0.003867 & Down \\
\hline TC09002626.hg.1 & -1.223346 & 0.001547 & Down \\
\hline TC01000854.hg.1 & -1.223146 & 0.00242 & Down \\
\hline TC07000408.hg.1 & -1.222936 & 0.001737 & Down \\
\hline TC01005446.hg.1 & -1.222469 & 0.002402 & Down \\
\hline TC21000963.hg.1 & -1.219803 & 0.00291 & Down \\
\hline TC0X001886.hg.1 & -1.219715 & 0.001036 & Down \\
\hline TC11000031.hg.1 & -1.218532 & 0.002754 & Down \\
\hline TC07000624.hg.1 & -1.217935 & 0.002001 & Down \\
\hline TC01006076.hg.1 & -1.217866 & 0.002136 & Down \\
\hline TC15001040.hg.1 & -1.217766 & 0.003343 & Down \\
\hline TC06000650.hg.1 & -1.216946 & 0.001062 & Down \\
\hline TC05001581.hg.1 & -1.216666 & 0.00138 & Down \\
\hline TC04002772.hg.1 & -1.216379 & 0.002777 & Down \\
\hline TC02003970.hg.1 & -1.215311 & 0.001808 & Down \\
\hline
\end{tabular}


Table 2 (continued)

\begin{tabular}{|c|c|c|c|}
\hline Probe set ID & Fold change & p-value & Gene feature \\
\hline TC10002734.hg.1 & -1.21518 & 0.003732 & Down \\
\hline TC10002413.hg.1 & -1.214979 & 0.003015 & Down \\
\hline TCOX001785.hg.1 & -1.214397 & 0.003416 & Down \\
\hline TC18000954.hg.1 & -1.214124 & 0.003768 & Down \\
\hline TCOX001752.hg.1 & -1.213315 & 0.002895 & Down \\
\hline TC02003848.hg.1 & -1.213273 & 0.000843 & Down \\
\hline TC01001745.hg.1 & -1.213018 & 0.001208 & Down \\
\hline TC03002074.hg.1 & -1.212781 & 0.001375 & Down \\
\hline TC01002757.hg.1 & -1.211987 & 0.001696 & Down \\
\hline TC02002040.hg.1 & -1.211577 & 0.002139 & Down \\
\hline TC21000403.hg.1 & -1.211543 & 0.002678 & Down \\
\hline TC17002504.hg.1 & -1.211528 & 0.002074 & Down \\
\hline TC02003570.hg.1 & -1.211373 & 0.000757 & Down \\
\hline TC01001703.hg.1 & -1.211313 & 0.002191 & Down \\
\hline TC05002704.hg.1 & -1.211017 & 0.002259 & Down \\
\hline TC05001346.hg.1 & -1.209244 & 0.002105 & Down \\
\hline TC07000864.hg.1 & -1.208029 & 0.002131 & Down \\
\hline TC03002933.hg.1 & -1.208009 & 0.002345 & Down \\
\hline TC02003270.hg.1 & -1.206919 & 0.003966 & Down \\
\hline TC17002837.hg.1 & -1.206485 & 0.003765 & Down \\
\hline TC08002366.hg.1 & -1.205822 & 0.001688 & Down \\
\hline TC06003509.hg.1 & -1.205272 & 0.000789 & Down \\
\hline TC07003058.hg.1 & -1.204848 & 0.003901 & Down \\
\hline TC09000783.hg.1 & -1.204566 & 0.001995 & Down \\
\hline TC09001972.hg.1 & -1.203884 & 0.000765 & Down \\
\hline TC02002065.hg.1 & -1.203643 & 0.002939 & Down \\
\hline TC15001919.hg.1 & -1.203339 & 0.003106 & Down \\
\hline TC07003162.hg.1 & -1.202549 & 0.002973 & Down \\
\hline TC15001221.hg.1 & -1.202261 & 0.003523 & Down \\
\hline TC21000290.hg.1 & -1.201768 & 0.002413 & Down \\
\hline TC04002818.hg.1 & -1.200872 & 0.002532 & Down \\
\hline TC06000891.hg.1 & -1.200617 & 0.002327 & Down \\
\hline TC03000459.hg.1 & -1.200421 & 0.00082 & Down \\
\hline Probe set ID & Fold change & p-value & Gene feature \\
\hline \multicolumn{4}{|c|}{$\begin{array}{l}\text { (F) Genes up/down-regulated in purified primary lung TCs compared with non- } \\
\text { purified primary lung TCS }\end{array}$} \\
\hline TCOM000020.hg.1 & -8.322621 & $2.70 E-05$ & Down \\
\hline TCOM000022.hg.1 & -2.0304 & $3.00 E-05$ & Down \\
\hline TC05002512.hg.1 & -1.586601 & $3.30 \mathrm{E}-05$ & Down \\
\hline TC04000160.hg.1 & -1.398875 & $3.50 \mathrm{E}-05$ & Down \\
\hline TCOM000023.hg.1 & -1.833263 & $3.80 E-05$ & Down \\
\hline TC03002425.hg.1 & -1.476136 & $4.60 E-05$ & Down \\
\hline TC01004110.hg.1 & -1.4988 & $4.80 \mathrm{E}-05$ & Down \\
\hline TC05003112.hg.1 & -1.883898 & $5.60 \mathrm{E}-05$ & Down \\
\hline TC07002463.hg.1 & -1.413454 & $6.10 \mathrm{E}-05$ & Down \\
\hline TC05003313.hg.1 & -1.291542 & $6.40 \mathrm{E}-05$ & Down \\
\hline TC02004623.hg.1 & -1.595285 & $7.70 \mathrm{E}-05$ & Down \\
\hline TC12002040.hg.1 & -1.484009 & $8.50 E-05$ & Down \\
\hline TC08000385.hg.1 & -1.344602 & $9.30 E-05$ & Down \\
\hline
\end{tabular}

between 10th and 30th passage. Both studies strongly suggest that it should be extremely careful to apply more than the 30th passage of the telocyte cell-line and RAW 264.7 cell line for preclinical research, in order to avoid the questions of data reliability.

$\mathrm{TCs}^{\mathrm{SV} 40}$ showed a dose-dependent response to challenges and therapies which are one of important functional properties of cells. We selected LPS as a stimulus of infection and inflammation and TNF $\alpha$ as an inflammatory mediator at different concentrations treated with or without signal pathway inhibitors and found the proliferative capacity of $\mathrm{TCs}^{\mathrm{SV} 40}$ declined with an increased concentration of LPS or TNF $\alpha$, similar to responses of primary TCs [23]. Phosphoinositide 3-kinase (PI3K) is a family of related intracellular signal transducer enzymes to phosphorylate the 3-position hydroxyl group of the inositol ring of phosphatidylinositol, perform cellular functions (e.g. cell growth, proliferation, differentiation, and survival), and regulate cell responses to drug therapy [24]. Glycogen synthase kinase 3 (GSK3) is a serine/threonine protein kinase to regulate the addition of phosphate molecules onto serine and threonine amino acid residues, carry out biological function (e.g. cellular proliferation, migration, glucose regulation, and apoptosis), and help cellular reprogramming for clinical cartilage repair [25]. $\mathrm{TCs}^{\mathrm{SV} 40}$ were sensitive to therapeutic effects of both PI3K and GSK3 inhibitors on prevention of LPS or TNF $\alpha$-reduced proliferation. Of those pathways, GSK3 seems more dominate in telocyte response to challenges. However, more basic and pre-clinical studies are still needed to ensure the safety and efficacy of gene-edited cells before clinical application. Standard and strict application for clinical research ethics must be carried out before the implementation of clinical research. We suggest that lung $\mathrm{TCs}^{\mathrm{SV} 40}$ can be applied for further studies to understand molecular mechanisms by which TCs communicate with other cells. 


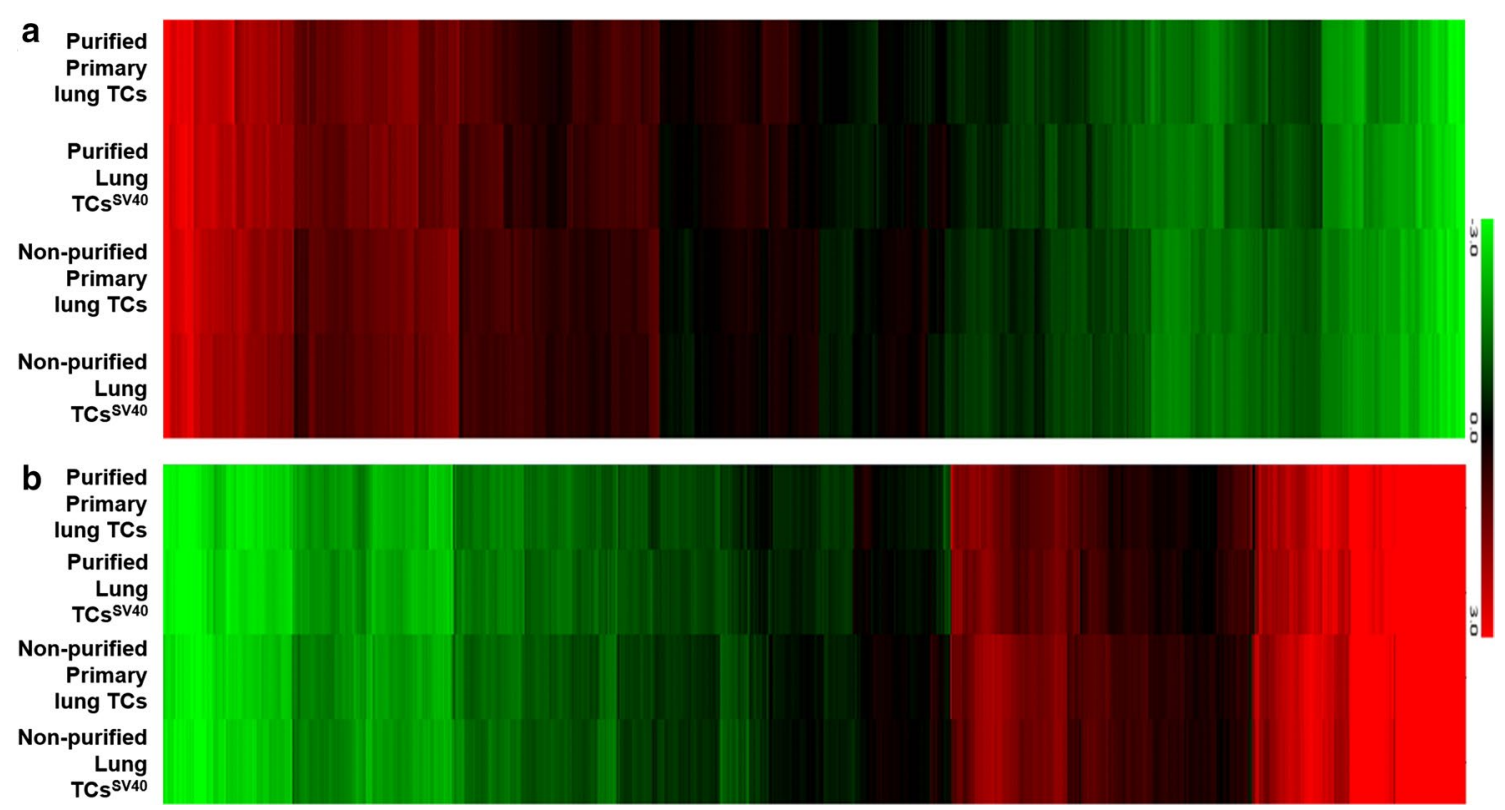

Fig. 4 Hotmap of differential Genes of primary TCS, SV40-transformed TCS, primary lung cells, and SV40-transformed primary lung cells. a Hotmap of differential mRNAs. b Hotmap of LncRNAs of primary TCs, SV40-transformed TCs, primary lung cells, and SV40-transformed primary lung cells

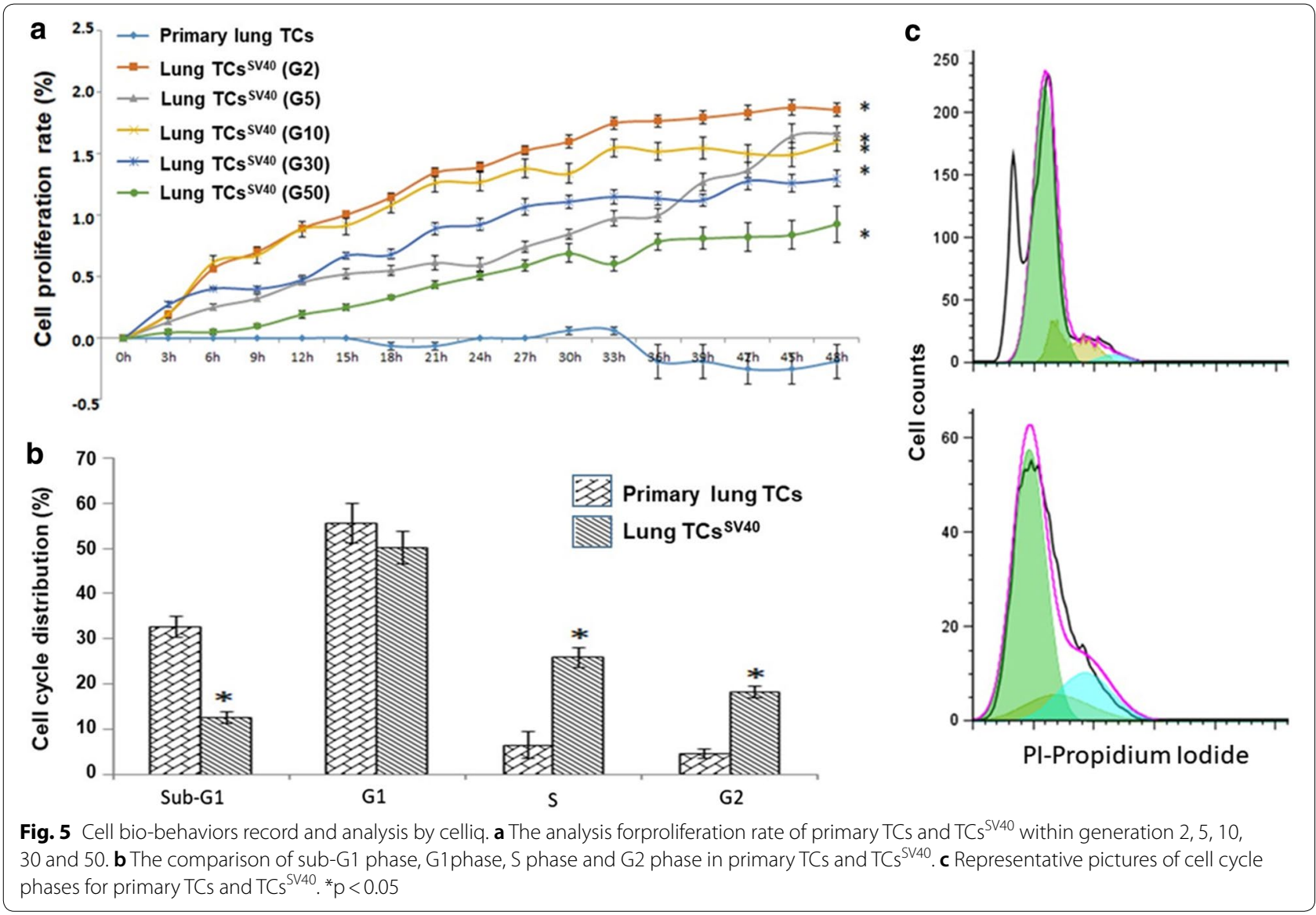



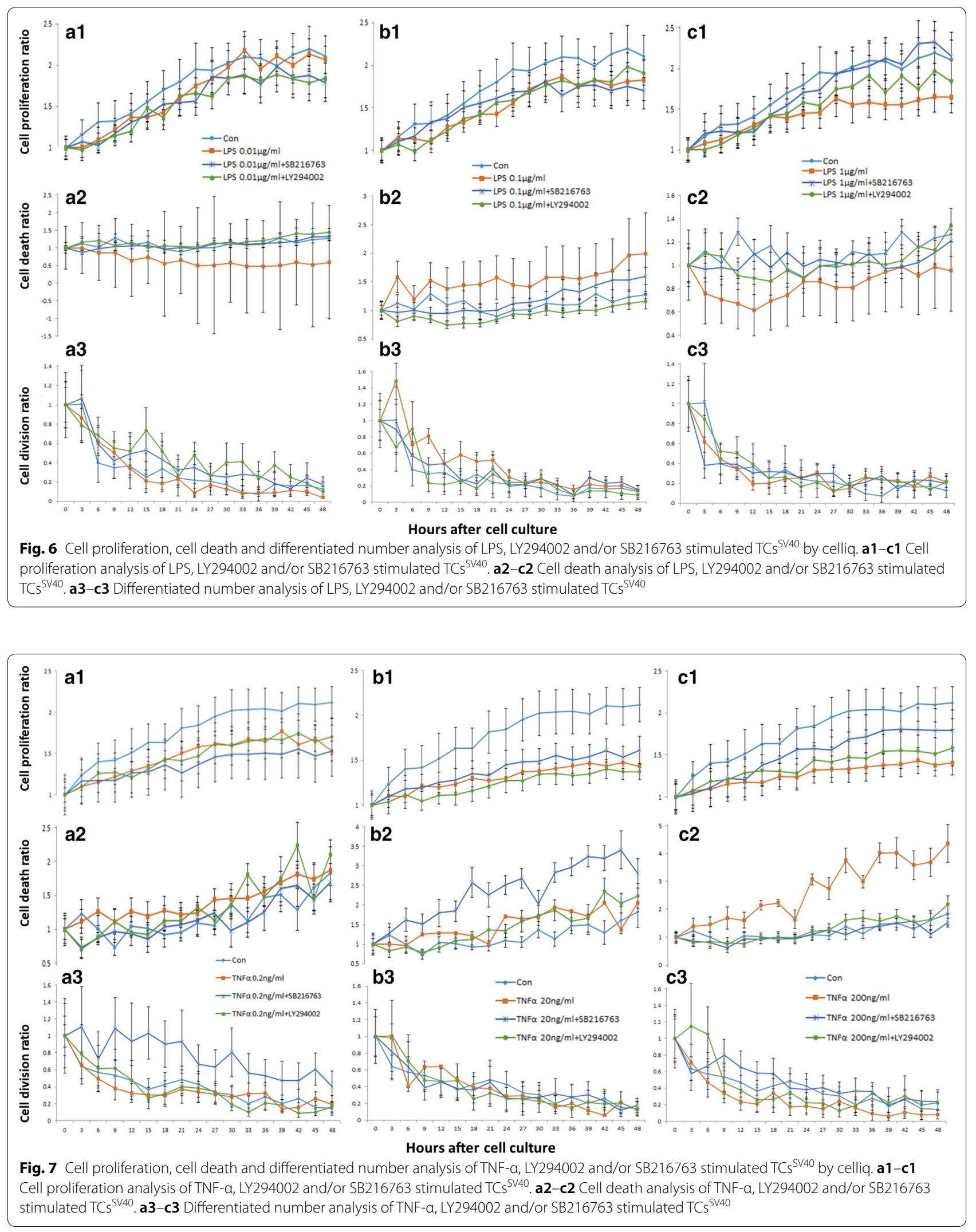


\section{Conclusion}

Lung TCs play important roles in maintenance of cellcell communication within the lung and pulmonary function. The present study initially established a mouse lung telocyte cell-line and evaluated the characteristics of TCs phenomes and functions by screening gene expression profiles, ultrastructure, cell biomarkers, and responses to challenges and therapies. We defined the comparability, characteristics and stability in various passages and optimized phenotypic and functional stability of telocyte lines for application. Thus, our data suggest that the newly established lung telocyte cell-line will provide an important tool to understand roles of TCs in maintenance of lung anatomy and functions and develop a new alterative of therapies for lung diseases.

\section{Additional files}

Additional file 1: Figure S1. Representative photos of cell bio-behaviors of primary TCs and TCs ${ }^{\mathrm{SV} 40}$ at 2, 5, 10, 20, 30, or 50 generations recorded for $0 \mathrm{~h}, 12,24,36 \mathrm{~h}$ and $48 \mathrm{~h}$ captured by celliq.

Additional file 2: Table S1. The profiles of transcriptional factor and IncRNA genes (Table S1) between primary lung TCs and TCs ${ }^{5 V 40}$ were compared and listed Tables 1 and 2, and the hotmap was shown in Fig. 4.

Additional file 3: Figure S2. Cell proliferation, cell death and differentiated number analysis of LY294002 or SB216763 stimulated TCs ${ }^{\text {SV40 }}$ by celliq.

Additional file 4: Figure S3. Cell bio-behaviors of TCs ${ }^{\mathrm{SV} 40}$ stimulated by LPS, LY294003 and SB216763 recorded by celliq.

Additional file 5: Figure S4. Cell bio-behaviors of TCs ${ }^{\mathrm{SV} 40}$ stimulated byTNF-a, LY294003 and SB216763 recorded by celliq.

\section{Abbreviations}

Tps: telocytes; Tps: telopodes; LPS: lipopolysaccharides; SV40: simian vacuolating virus 40 small and large antigen; TEM: transmission electron microscopy; TNF: tumor necrosis factor; SPSS: Statistical Package for the Social Sciences; ANOVA: one-way analysis of variance; PDGFRa: platelet-derived growth factor receptor a.

\section{Authors' contributions}

DS, MX and XW designed the study and patient specimen collection. DS, MX $\mathrm{RM}$ and $\mathrm{YZ}$ completed the experimental process, data collection, literature search and generation of figures. DS, MX and XW wrote and edited the manuscript. RQ, DW and HF provided the experimental technical and laboratory facilities support. All authors reviewed the manuscript. All authors read and approved the final manuscript.

\section{Acknowledgements}

We wish to thank Mr. Hongjian Gao for his assistance in preparing cell samples for electron microscope and immuno-electron microscope detection.

\section{Competing interests}

The authors declare that they have no competing interests.

\section{Availability of data and materials}

Not applicable.

\section{Consent for publication}

Not applicable.

\section{Ethics approval and consent to participate}

This study was approved by the Fudan University Ethical Committee for animal experiments.

\section{Funding}

This work was supported by Grants from the National Natural Science Foundation of China (81700008).

\section{Publisher's Note}

Springer Nature remains neutral with regard to jurisdictional claims in published maps and institutional affiliations.

\section{Author details}

${ }^{1}$ Zhongshan Hospital Institute of Clinical Science, Shanghai Institute of Clinical Bioinformatics, Shanghai Engineering Research for Al Technology for Cardiopulmonary Diseases, Shanghai, China. ${ }^{2}$ Center for Tumor Diagnosis and Therapy, Zhongshan Hospital Jinshan Hospital, Shanghai Medical College, Fudan University, Shanghai, China. ${ }^{3}$ Department of Anesthesiology, Zhongshan Hospital, Shanghai, China. ${ }^{4}$ Department of Anesthesiology, Minhang Branch, Zhongshan Hospital, Fudan University, Shanghai, China.

Received: 1 March 2019 Accepted: 3 April 2019

Published online: 15 May 2019

\section{References}

1. Song D, Cretoiu D, Cretoiu SM, et al. Telocytes and lung disease. Histol Histopathol. 2016:31(12):1303-14.

2. Wang J, Jin M, Ma WH, et al. The history of telocyte discovery and understanding. Adv Exp Med Biol. 2016;913:1-21.

3. Shoshkes-Carmel M, Wang YJ, Wangensteen KJ, et al. Subepithelial telocytes are an important source of Wnts that supports intestinal crypts. Nature. 2018;557(7704):242-6.

4. Song D, Cretoiu D, Zheng M, et al. Comparison of chromosome 4 gene expression profile between lung telocytes and other local cell types. J Cell Mol Med. 2016;20(1):71-80.

5. Wang J, Ye L, Jin M, et al. Global analyses of chromosome 17 and 18 genes of lung telocytes compared with mesenchymal stem cells, fibroblasts, alveolar type II cells, airway epithelial cells, and lymphocytes. Biol Direct. 2015;10:9.

6. Sun X, Zheng M, Zhang M, et al. Differences in the expression of chromosome 1 genes between lung telocytes and other cells: mesenchymal stem cells, fibroblasts, alveolar type II cells, airway epithelial cells and lymphocytes. J Cell Mol Med. 2014;18(5):801-10.

7. Zheng $M$, Sun $X$, Zhang $M$, et al. Variations of chromosomes 2 and 3 gene expression profiles among pulmonary telocytes, pneumocytes, airway cells, mesenchymal stem cells and lymphocytes. J Cell Mol Med. 2014;18(10):2044-60

8. Rusu MC, Cretoiu D, Vrapciu AD, et al. Telocytes of the human adult trigeminal ganglion. Cell Biol Toxicol. 2016;32(3):199-207.

9. Song D, Cretoiu D, Wang X. Mitochondrial DNA in telocytes. Adv Exp Med Biol. 2017:1038:55-70.

10. Ye $L$, Song $D$, Jin $M$, et al. Therapeutic roles of telocytes in OVA-induced acute asthma in mice. J Cell Mol Med. 2017:21(11):2863-71.

11. Zheng $Y$, Wang $X$. Roles of telocytes in the development of angiogenesis. Adv Exp Med Biol. 2016:913:253-61.

12. Shi L, Dong N, Chen C, et al. Potential roles of telocytes in lung diseases. Semin Cell Dev Biol. 2016;55:31-9.

13. Gammage PA, Viscomi C, Simard ML, et al. Genome editing in mitochondria corrects a pathogenic mtDNA mutation in vivo. Nat Med. 2018;24(11):1691-5.

14. Wang W, Wang X. Single-cell CRISPR screening in drug resistance. Cell Biol Toxicol. 2017;33(3):207-10

15. Blum W, Pecze L, Felley-Bosco E, et al. Establishment of immortalized murine mesothelial cells and a novel mesothelioma cell line. Vitro Cell Dev Biol Anim. 2015;51(7):714-21.

16. Zheng Y, Li H, Manole CG, et al. Telocytes in trachea and lungs. J Cell Mol Med. 2011;15(10):2262-8. 
17. Zhu Y, Zheng M, Song D, et al. Global comparison of chromosome $X$ genes of pulmonary telocytes with mesenchymal stem cells, fibroblasts, alveolar type II cells, airway epithelial cells, and lymphocytes. J Transl Med. 2015;13:318.

18. Blum W, Schwaller B. Calretinin is essential for mesothelioma cell growth/survival in vitro: a potential new target for malignant mesothelioma therapy? Int J Cancer. 2013;133(9):2077-88.

19. Toscano MG, van der Velden J, van der Werf S, et al. Generation of a vero-based packaging cell line to produce SV40 gene delivery vectors for use in clinical gene therapy studies. Mol Ther Methods Clin Dev. 2017;6:124-34.

20. Ando D, Meyer K. Gene editing: regulatory and translation to clinic. Hematol Oncol Clin North Am. 2017:31 (5):797-808.
21. Lux CT, Scharenberg AM. Therapeutic gene editing safety and specificity. Hematol Oncol Clin North Am. 2017;31(5):787-95.

22. Taciak B, Bialasek M, Braniewska A, et al. Evaluation of phenotypic and functional stability of RAW 264.7 cell line through serial passages. PLoS ONE. 2018;13(6):e0198943.

23. Chi C, Jiang XJ, Su L, et al. In vitro morphology, viability and cytokine secretion of uterine telocyte-activated mouse peritoneal macrophages. J Cell Mol Med. 2015;19(12):2741-50.

24. LV J, Wang L, Shen $H$, et al. Regulatory roles of OASL in lung cancer cell sensitivity to Actinidia chinensis Planch root extract (acRoots). Cell Biol Toxicol. 2018;34(3):207-18.

25. Driessen BJH, Logie C, Vonk LA. Cellular reprogramming for clinical cartilage repair. Cell Biol Toxicol. 2017;33(4):329-49. 